UNIVERSITAT POLITĖCNICA DE VALÈNCIA

DEPARTAMENTO DE QUÍMICA

INSTITUTO UNIVERSITARIO MIXTO DE TECNOLOGÍA QUÍMICA

(UPV-CSIC)

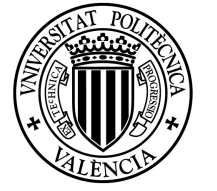

UNIVERSITAT

POLITÉCNICA

DE VALĖNCIA

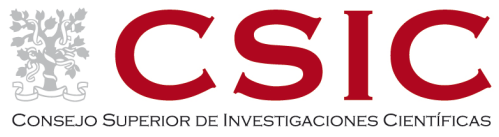

DOCTORAL THESIS

\title{
Model Studies on the Photorepair of (6-4) Dimeric Lesions of DNA
}

\section{Ana Belén Fraga Timiraos}

Valencia, January 2019

Directors:

Prof. Miguel A. Miranda Alonso

Dra. Virginie Lhiaubet-Vallet 



\section{CERTIFICATION}

Miguel Ángel Miranda Alonso, Full Professor of the Universitat Politècnica de València (UPV) and Virginie Lhiaubet-Vallet, Tenured Scientist of the CSIC,

CERTIFY that the Doctoral Thesis entitled "Models Studies on the Photorepair of (6-4) Dimeric Lesions of DNA" has been developed by Ana Belén Fraga Timiraos under their supervision in the Instituto Universitario Mixto de Tecnología Química (UPV-CSIC). 

A Marcos e meus pais 

Outline 



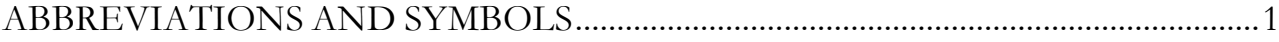

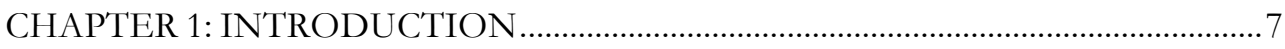

1.1. PHOTOPHYSICS AND PHOTOCHEMISTRY ........................................................

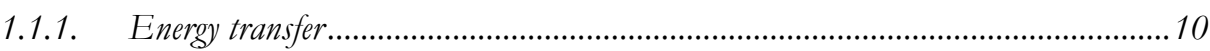

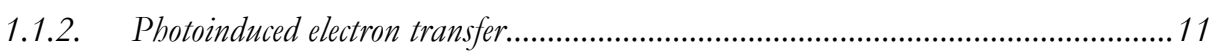

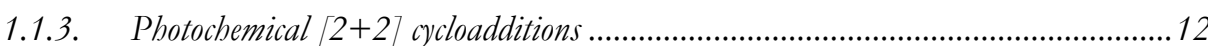

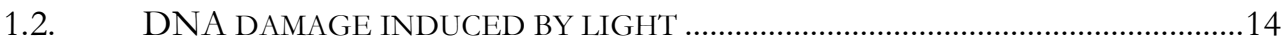

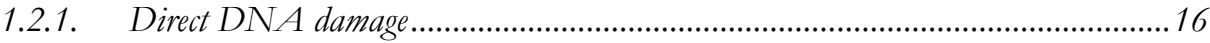

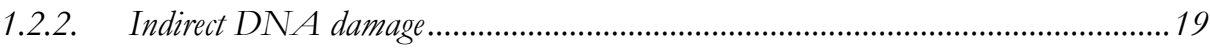

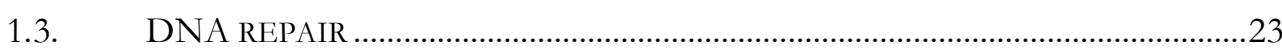

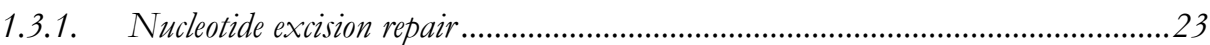

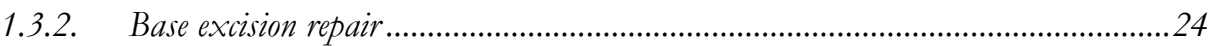

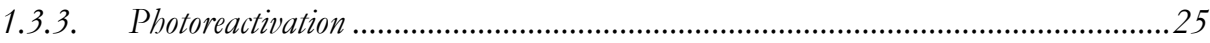

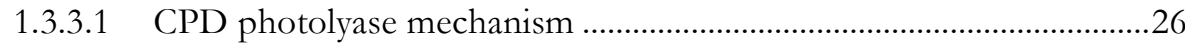

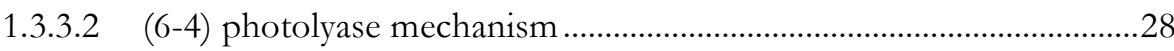

1.3.3.2.1 Investigation of the (6-4) repair mechanism with model compounds.

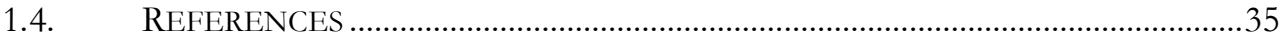

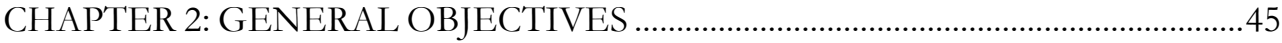

CHAPTER 3: PHOTOREDUCTION OF AN AZETIDINE MODEL OF THE (6-

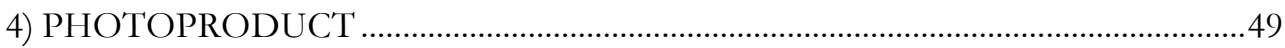

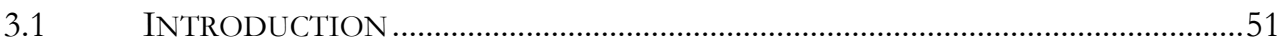

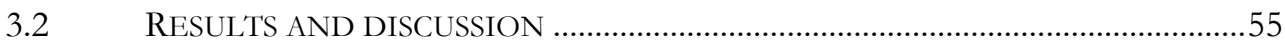

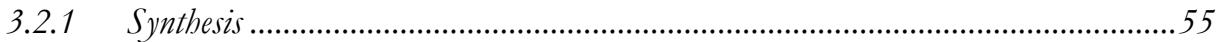

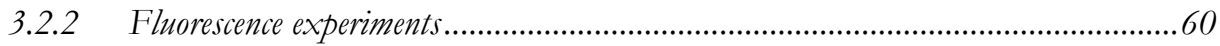

3.2.3 Electrochemical measurements ..........................................................................68

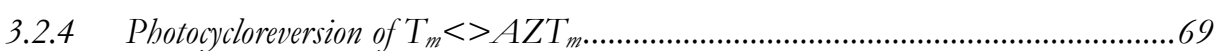

3.2.5 Quantum-chemistry determination of the photoreductive properties of $T<>A Z T$.... 71 
3.2.6 Decomposition mechanisms of $T<>A Z T$ radical anion....................................... 72

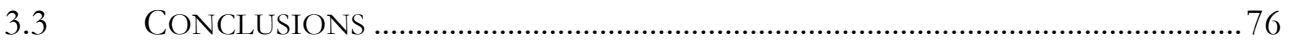

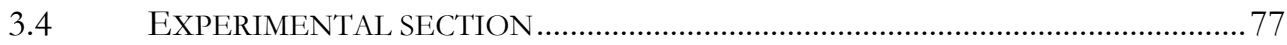

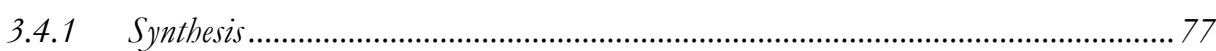

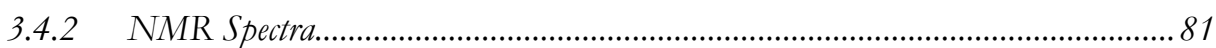

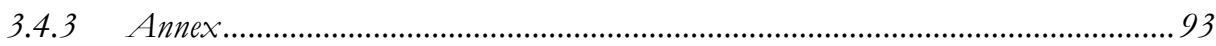

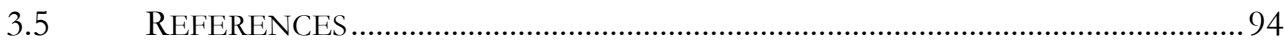

CHAPTER 4: STUDY OF AZETIDINES CYCLOREVERSION BY OXIDATIVE

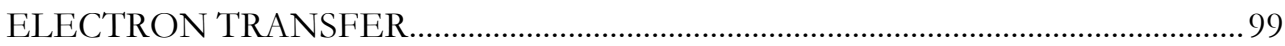

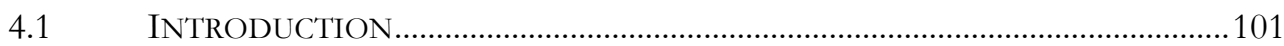

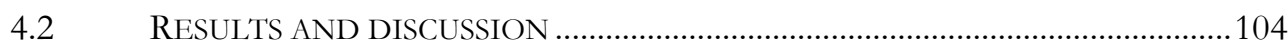

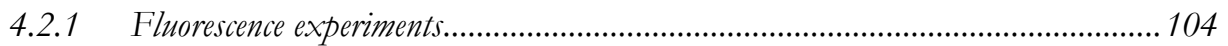

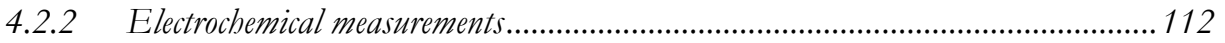

4.2.3 Photocycloreversion for $T_{m}<>A Z T_{m}$ model ......................................................113

4.2.4 Quantum-chemistry determination of the photooxidative properties for $T_{m}<>A Z T_{m}$ model ...............................................................115

4.2.5 Decomposition mechanism of $T<>$ AZT radical cation ........................................116

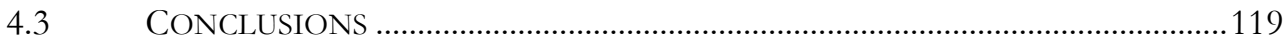

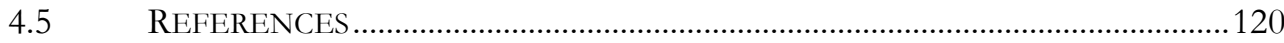

CHAPTER 5: PHOTOREPAIR OF THYMINE DERIVED CYCLOBUTANES

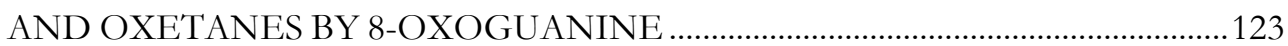

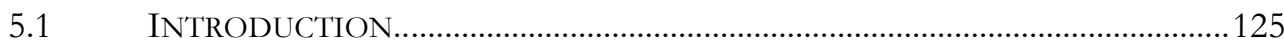

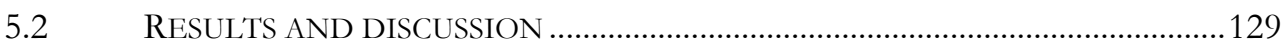

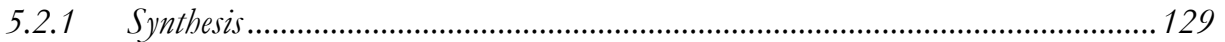

5.2.2 Photocycloreversion....................................................................................... 132

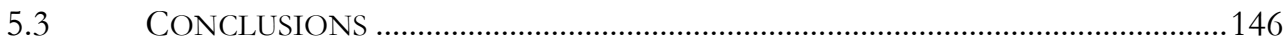

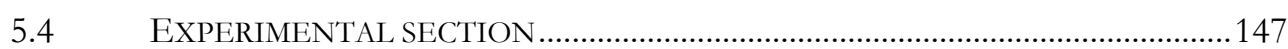

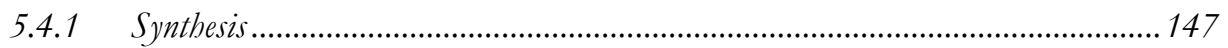




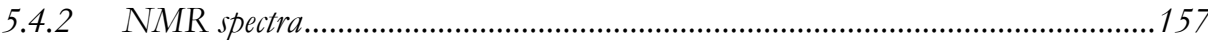

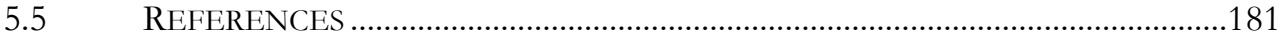

CHAPTER 6: 8-OXOGUANINE AS A POTENTIAL INTRINSIC PHOTOSENSITIZER FOR THE REPAIR OF T(6-4)C PHOTOPRODUCT........187

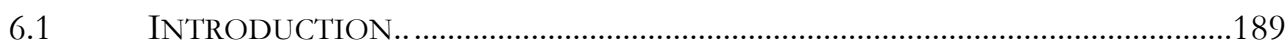

6.2 RESULTS AND DISCUSSION ………………………………………….....192

6.2.1 Synthesis of AZT........................................................................................192

6.2.2 Photorepair of AZT by OG .......................................................................196

6.2.3 Photorepair of AZT by photolyases..................................................................201

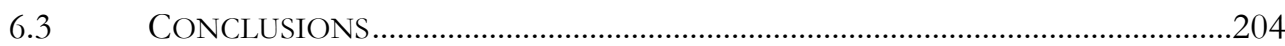

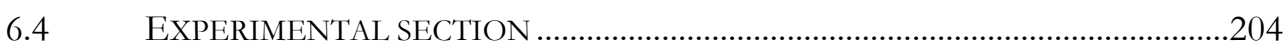

6.4.1 Oligonucleotide synthesis ..............................................................................204

6.4.2 Photochemical preparation of AZT oligonucleotide ……………………………...205

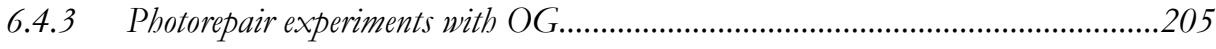

6.4.4 Photorepair experiments with photolyases ..........................................................206

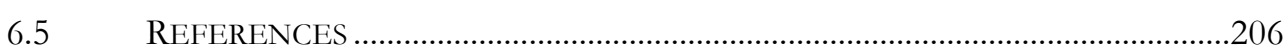

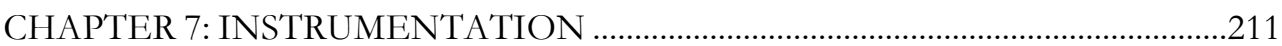

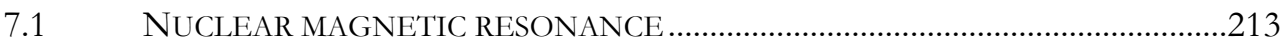

7.2 ABSORPTION MEASUREMENTS...................................................................213

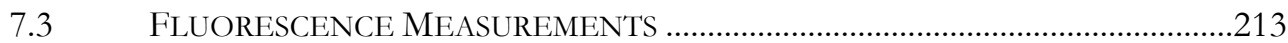

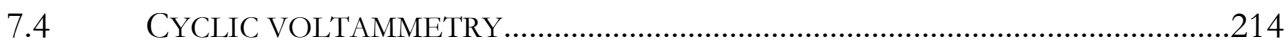

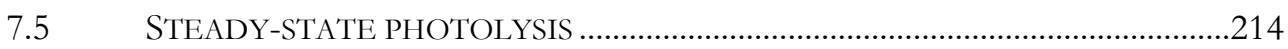

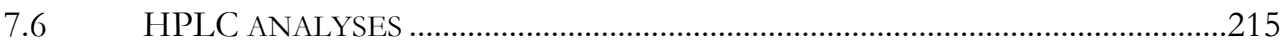

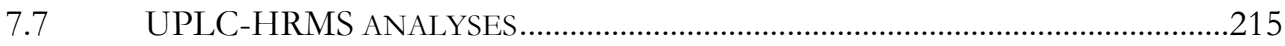

7.8 FEMTOSECOND TRANSIENT ABSORPTION SPECTROSCOPY ...........................216

7.9 QUANTUM-CHEMISTRY GROUND-STATE COMPUTATIONS............................217

CHAPTER 8: GENERAL CONCLUSIONS ……………………………………….....219 
Abbreviations and Symbols 

Abbreviations and Symbols

\begin{tabular}{|c|c|}
\hline A & Acceptor \\
\hline $\mathrm{ACE}$ & Acenaphthene \\
\hline $\mathrm{Ac}_{2} \mathrm{O}$ & Acetic anhydride \\
\hline $\mathrm{AcOH}$ & Acetic acid \\
\hline $\mathrm{AcONa}$ & Sodium acetate \\
\hline AIP & Adiabatic ionization potential \\
\hline AQ & Anthraquinone \\
\hline AQS & Anthraquinone-2-sulfonate \\
\hline azaU & 6-azaUracil \\
\hline BER & Base excision repair \\
\hline $\mathrm{BnOH}$ & Benzyl alcohol \\
\hline $\mathrm{BP}$ & Benzophenone \\
\hline CASPT2 & $\begin{array}{l}\text { Complete-active-space self-consistent field second- } \\
\text { order perturbation }\end{array}$ \\
\hline CASSCF & Complete-active space self-consistent field \\
\hline $\mathrm{CDCl}_{3}$ & Deuterated chloroform \\
\hline CDI & 1,1'-Carbonyldiimidazole \\
\hline CHRY & Chrysene \\
\hline CIDNP & Chemically induced dynamic nuclear polarization \\
\hline CPD & Cyclobutane pyrimidine dimer \\
\hline CRIP & Contact radical ion pair \\
\hline $\mathrm{D}$ & Donor \\
\hline $\mathrm{DABCO}$ & 1,4-Diazabicyclo[2.2.2]octane \\
\hline DCA & 9,10-Dicyanoanthracene \\
\hline $\mathrm{DCN}$ & 1,4-Dicyanonaphthalene \\
\hline DDQ & 2,3-dichloro-5,6-dicyano-1,4-quinone \\
\hline Dew & Dewar valence isomer \\
\hline DFT & Density functional theory \\
\hline
\end{tabular}




\begin{tabular}{|c|c|}
\hline DMA & N,N-dimethylaniline \\
\hline DMAP & 4-(Dimethylamino)pyridine \\
\hline $\mathrm{DMF}$ & Dimethylformamide \\
\hline DMSO-d6 & Dimethylsulfoxide \\
\hline DMT & 4,4'-Dimethoxytrityl \\
\hline DNA & Deoxyribonucleic acid \\
\hline $\mathrm{E}$ & Energy \\
\hline EA & Electron affinity \\
\hline EDC & 1-Ethyl-3-(3-dimethylaminopropyl)carbodiimide \\
\hline EET & Excess electron transfer \\
\hline ESI & Electrospray ionization \\
\hline ET & Electron transfer \\
\hline $\mathrm{Et}_{3} \mathrm{~N}$ & Triethylamine \\
\hline $\mathrm{EtOH}$ & Ethanol \\
\hline $\mathrm{F}$ & Fluorescence \\
\hline FAD & Flavin adenine dinucleotide \\
\hline FapyG & 2,6-Diamino-4-hydroxy-5 formamidopyrimidine \\
\hline ForU & 5-Formyluracil \\
\hline Fs-TAS & Femtosecond transient absorption spectroscopy \\
\hline GGR & Global genome repair \\
\hline 8-HDF & 8-Hydroxy-5-deazaflavin \\
\hline His & Histidine \\
\hline $\mathrm{HmU}$ & 5-Hydroxymethyluracil \\
\hline $\mathrm{HOMO}, \mathrm{HO}$ & Highest occupied molecular orbital \\
\hline HPLC & High-performance liquid chromatography \\
\hline HRMS & High-resolution mass spectroscopy \\
\hline IC & Internal conversion \\
\hline $\operatorname{Im}$ & Imidazole \\
\hline
\end{tabular}


Abbreviations and Symbols

\begin{tabular}{|c|c|}
\hline IP & Ionization potential \\
\hline ISC & Intersystem crossing \\
\hline LIIC & Linear interpolation of internal coordinates \\
\hline LUMO, LU & Lowest unoccupied molecular orbital \\
\hline $\mathrm{MeCN}$ & Acetonitrile \\
\hline $\mathrm{MeOH}$ & Methanol \\
\hline MEP & Minimum energy path \\
\hline $1-\mathrm{MN}$ & 1-Methoxynaphthalene \\
\hline $2-\mathrm{MN}$ & 2-Methoxynaphthalene \\
\hline MTHF & Methenyltetrahydrofolate \\
\hline $\mathrm{NaPi}$ & Sodium phosphate buffer \\
\hline NBS & N-Bromosuccinimide \\
\hline NER & Nucleotide excision repair \\
\hline NHE & Normal hydrogen electrode \\
\hline NMR & Nuclear magnetic resonance \\
\hline $\mathrm{OD}$ & Optical density \\
\hline $\mathrm{OG}$ & 8-oxoGuanine \\
\hline $\mathrm{P}$ & Phosphorescence \\
\hline PBS & Phosphate-buffered saline \\
\hline PET & Photoinduced electron transfer \\
\hline 6-4PP & (6-4) Photoproduct \\
\hline PS & Photosensitizer \\
\hline RNA & Ribonucleic acid \\
\hline ROS & Reactive oxygen species \\
\hline $\mathrm{rt}$ & Room temperature \\
\hline $\mathrm{S}_{0}$ & Ground state \\
\hline $\mathrm{S}_{1}$ & Singlet excited state \\
\hline SSET & Singlet-singlet energy transfer \\
\hline
\end{tabular}


Abbreviations and Symbols

SSRIP

$\mathrm{T}_{1}$

TBAF

TBDMSCl

TBTU

TCR

TEAA

TFA

THF

TLC

TMB

TMPD

TOF-MS

TS

TTET

UPLC

UV

VIP

ZPVE

$\delta$

$\varepsilon$

$\phi$

$\lambda$

$\tau$
Solvent separated radical ion pair

Triplet excited state

Tetrabutylammonium fluoride

tert-Butyldimethylsilyl chloride

$N, N, N$ ',N'-tetramethyl-O-(benzotriazol-1-

yl)uronium tetrafluoroborate

Transcription-coupled repair

Triethylammonium acetate

Trifluoroacetic acid

Tetrahydrofuran

Thin-layer chromatography

N,N,N',N'-Tetramethylbenzidine

N,N,N',N'-Tetramethyl-1,4-phenyldiamine

Time-of-flight mass spectrometry

Transition state

Triplet-triplet energy transfer

Ultra performance liquid chromatography

Ultraviolet light

Vertical ionization potential

Zero-point vibrational energy

Chemical shift

Molar absorption coefficient

Quantum yield

Wavelength

Lifetime 
Chapter 1:

Introduction 



\subsection{Photophysics and photochemistry}

Molecular photochemistry is the discipline which studies chemical reactions induced by the absorption of UV-Vis (200 - $700 \mathrm{~nm})$ and infrared light (700 - $2500 \mathrm{~nm})$. Organisms have evolved to take advantage and respond to light in numerous ways such as in photosynthesis, vision or the formation of Vitamin D. Understanding how these and other systems convert photons into energy or biological signals is an important issue for the chemical biology community to establish the mechanistic details of these processes.

The process starts when a chromophore which contains an organic functional group such as an alkene, aromatic ring, ketone or enone, absorbs light (hv) from its ground state $\left(\mathrm{S}_{0}\right)$. Upon aborption of a photon, the molecule may promote an electron from the highest occupied molecular orbital (HOMO, HO) to the lowest unoccupied molecular orbital (LUMO, LU), reaching an excited electronic state. Therefore, this excited species in the unstable singlet excited state $\left(S_{n}\right.$ or $\left.S_{1}\right)$ tends to deactivate rapidly to the stable ground state $\left(\mathrm{S}_{0}\right)$ by different pathways as shown in the Jablonski diagram $^{1}$ (Figure 1.1). This can be through non-radiative (internal conversion, IC) or radiative processes (fluorescence, F) by generation of light emission. Another pathway from $\mathrm{S}_{1}$ involves forbidden transitions denominated intersystem crossing (ISC), in this case a change in the spin multiplicity gives rise to a triplet excited state $\left(T_{n}\right.$ or $\left.T_{1}\right)$. After that, the generated triplet excited state $T_{1}$ will deactivate to the $S_{0}$ through nonradiative (ISC) or radiative processes (phosphorescence, $\mathrm{P}$ ).

However, deactivation of the excited states does not always lead to the original molecule in its ground state as photochemical processes might also occur to yield primary photoproducts that are chemically different from the original reactant. Photoreactions leading to new products can be efficient only if they are faster than the competing photophysical processes. In the presence of other substrates, intermolecular 
processes can take place such as singlet-singlet or triplet-triplet energy transfer (SSET and TTET, respectively), electron transfer, photocycloaddition, etc.

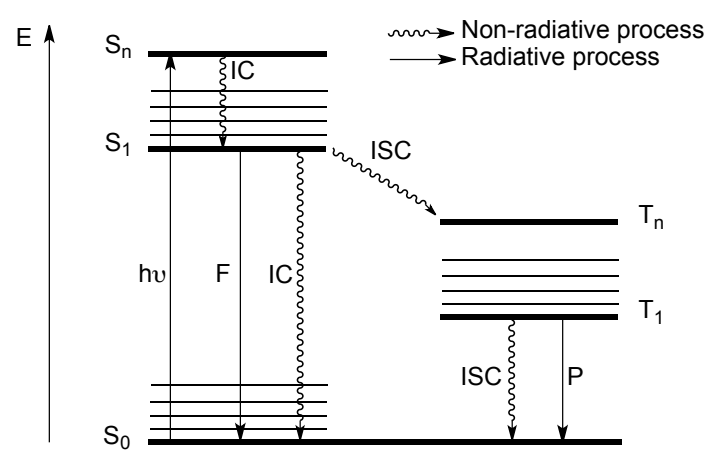

Figure 1.1. Jablonski diagram represents the electronic states of a molecule and the transitions that can occur after absorption of light.

In this context, it is important to gain knowledge in the physical properties of the different chromophores and the photoreactions they can be involved in. This doctoral thesis is centered on the photorepair mechanism of different DNA damage models induced by UV. For this reason, it is important to detail intermolecular processes such as energy transfer, electron transfer and photocycloadditions triggered by UV light.

\subsubsection{Energy transfer}

In this intermolecular process, the excitation energy of an excited molecule $\mathrm{D}^{*}$, i.e. the energy donor, is transferred to a neighboring molecule A, i.e. the energy acceptor, which reaches an electronic excited state (Scheme 1.1). Thus, energy transfer permits electronic excitation of molecules A that do not absorb the incident light. The multiplicity of $\mathrm{D}^{*}$ and $\mathrm{A}^{*}$ will stipulate if it is singlet-singlet energy transfer (SSET) or triplet-triplet energy transfer (TTET).

$$
D^{*}+A \rightarrow D+A^{*}
$$

Scheme 1.1. 
For this process to occur, the energy level of the excited state of $\mathrm{D}^{*}$ has to be higher than that of $\mathrm{A}^{*}$ (Equation 1.1) and the time scale of the energy transfer must be faster than the intrinsic lifetime of $\mathrm{D}^{*}$.

$$
\Delta G \approx \Delta H=E^{D *}-E^{A *}
$$

where $\Delta G$ is the variation of the free energy of the process and $E^{D *}$ and $E^{A *}$ are the energies of the excited state (singlet excited state or triplet excited state) of the donor and the acceptor, respectively. These processes generally take place following two mechanisms: the coulombic (Förster type), or the exchange electronic (Dexter type). ${ }^{2}$ The Förster type involves a dipole-dipole interaction and acts at long distances (up to $100 \AA)$. By contrast, the Dexter type mechanism requires an effective overlap between orbitals of the donor and the acceptor and thus takes place at short distances (under $10 \AA)$.

\subsubsection{Photoinduced electron transfer}

One of the most basic reactions studied in organic chemistry is photoinduced electron transfer (PET), where an electron rich donor (D) or an electron deficient acceptor (A) in its excited state transfers an electron to another acceptor or donor in its ground state. (Scheme 1.2). ${ }^{3}$ This generates the radical anion of the acceptor and the radical cation of the donor through the formation of a contact radical ion pair (CRIP), which is also known as an exciplex. Depending on other factors, such as interaction with the solvent, the ion pair can undergo solvation to become a solvent separated radical ion pair (SSRIP) or can directly undergo back electron transfer to the ground state reactants. The SSRIP can also suffer back electron transfer to the ground state acceptor and donor, or undergo solvent cage escape to generate free radical ions in solution. 


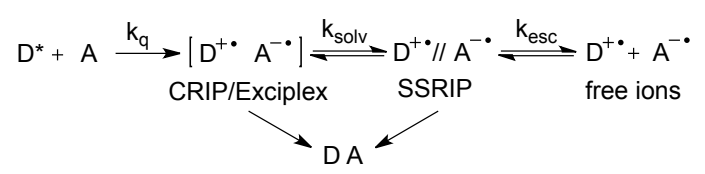

Scheme 1.2. Common PET pathways in non-viscous solvents.

The thermodynamics of these processes is controlled by the oxidation and reduction potentials of the species involved in the reaction, as well as by the energy of the excited state of the molecule that absorbs light. So, for simplicity the free energy associated with PET is given by the Rehm-Weller equation ${ }^{4}$ (1.2).

$$
\Delta G_{c t}=23.06\left(E_{o x}^{D}-E_{r e d}^{A}-\frac{q^{2}}{\varepsilon r}\right)-E^{*}
$$

where $E_{o x}^{D}$ and $E_{r e d}^{A}$ are the oxidation and reduction potentials of the donor and

acceptor, respectively, $\frac{q^{2}}{\varepsilon r}$ is a coulombic interaction term, and $E^{*}$ is the excited state energy of the photosensitizer (singlet excited state or triplet excited state). Finally, the term 23.06 is the Faraday constant given in $\mathrm{kcal} / \mathrm{mol}$. This equation informs about the spontaneity of a PET reaction in solution. Therefore, it can be estimated, with a certain approximation, if the process is going to be favorable ( $\Delta G<0$, exergonic) or, for the contrary, unfavorable $(\Delta \mathrm{G}>0$, endergonic).

\subsubsection{Photochemical [2+2] cycloadditions}

The [2+2] cycloaddition reaction is the most applied reaction in organic synthesis for the formation of compounds containing a four-membered ring. ${ }^{5}$ This way, cyclobutanes can be obtained by addition of an alkene in its ground state to a different photoexcited alkene (photocycloaddition) or by dimerization of two identical alkenes, one of them previously excited (photodimerization). ${ }^{6}$ The photoreaction mechanism depends on the pathway to generate the photoexcited alkene (Scheme 1.3). Direct excitation can lead to the alkene lowest excited state $S_{1}$, from which [2+2] cycloaddi- 
tion can occur (Scheme 1.3, pathway a). Usually, it happens when the alkenes absorb at wavelengths higher than $270 \mathrm{~nm}$ and their $S_{1}$ is of $\pi \pi^{*}$ nature such as for aryl substituted alkenes. If this is not the case, the excitation energy is readily dissipated leading to side reactions such as cis/trans isomerization and, thus, to low yields for the cycloaddition.

In the specific case of $\alpha, \beta$-unsaturated carbonyl compounds, the $S_{1}$ is of n $\pi^{*}$ nature but a reactive $\mathrm{T}_{1}$ is usually efficiently populated through high ISC. Thus, combination of the enone in its triplet excited state with an alkene leads to 1,4-biradical intermediate, from which the cyclobutane is formed (Scheme 1.3, pathway b). ${ }^{7}$

An alternative pathway is to generate the $T_{1}$ excited state of the alkene by a photosensitized reaction (Scheme 1.3, pathway c). In this case, energy transfer occurs when the triplet energy of the alkene is lower than the triplet energy of the photosensitizer (PS). Therefore, as for $\alpha, \beta$-unsaturated carbonyl compounds, the cycloaddition takes place from the $\mathrm{T}_{1}$ of the alkene. ${ }^{8}$

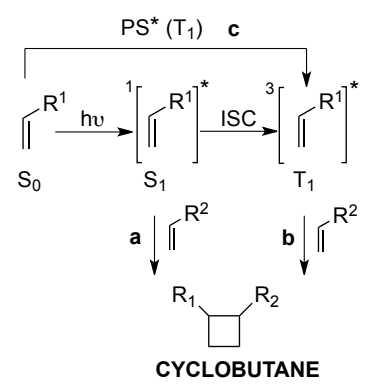

Scheme 1.3. Different pathways for the [2+2] cycloaddition reaction between alkenes: a) [2+2] cycloaddition from the $\mathrm{S}_{1}$; b) [2+2] cycloaddition from the $\mathrm{T}_{1}$ and c) photosensitized [2+2] cycloaddition.

Small heterocycles such as the four-membered oxetane ring can also be obtained by [2+2] photocycloaddition. In this case, a photoexcited carbonyl group and an alkene are involved in the photoreaction denominated Paternò-Büchi reaction.6, 9-10 
The carbonyl compound is the light-absorbing chromophore, which reaches a $S_{1}$ or $T_{1}$ $\left(\mathrm{n} \pi^{*}\right)$ and, then, adds to the alkene (Scheme 1.4). The involvement of 1,4-biradical species derived from carbonyl triplets were studied spectroscopically and by trapping with biradical quenchers. ${ }^{11}$ This $[2+2]$ photoreaction competes with TTET and is favored for $n \pi^{*}$ triplets when the triplet energy of the alkene is comparable to or higher than that of the carbonyl group.

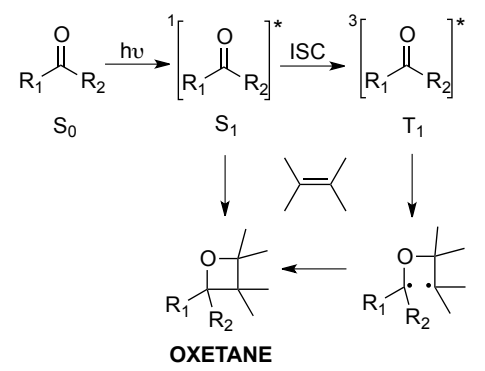

Scheme 1.4. Mechanism involved in the Paternò-Büchi reaction.

As mentioned above, four-membered ring formation is well known through [2+2] photocycloadditions of alkenes to $\mathrm{C}=\mathrm{C}$ or $\mathrm{C}=\mathrm{O}$ bonds, but similar cycloadditions to imines $(\mathrm{C}=\mathrm{N})$ are less common. ${ }^{12}$ The structural requirements to lead to a four-membered azetidine ring are discussed in the Chapter 3.

\subsection{DNA damage induced by light}

Among the radiation emitted by the Sun that reaches the Earth's surface only 6.1\% resides in the UV range, which is divided into UVC (100-290 nm), UVB (290$320 \mathrm{~nm}$ ), and UVA (320-400 nm). Fortunately, ozone and oxygen completely block UVC radiation and absorb the majority (approximately 95\%) of UVB. ${ }^{13}$

The incident UV radiation has important implications for human health, among them, skin cancer is one of the most harmful effects of overexposure. ${ }^{14}$ In this context, UV is considered as a carcinogenic and mutagenic component of the solar irradiation spectrum by its ability to cause DNA damage, promotion and progression in 
tumorogenesis. ${ }^{15}$ Thus, it is important to understand the mechanisms involved in skin cancer by identifying the different DNA photoproducts and their specific contribution to mutagenesis to find relevant prevention strategies.

Regarding to harmful effects induced by UV wavelengths onto DNA, UVC would be the most effective waveband for the induction of DNA photoproducts because the DNA molecule shows the maximum absorption at $260 \mathrm{~nm}$; however, as mentioned above, these wavelengths are entirely blocked by the Earth's atmosphere. Additionally, although the energy of incident UVB and UVA photons is high enough to generate DNA damage, UVA is capable of penetrating the skin more efficiently than UVB, reaching the basal layers where melanocytes and dividing stem cells are located in the dermis (Figure 1.2). ${ }^{16}$ Moreover, UVB is considered the most mutagenic and carcinogenic since it is absorbed directly by the DNA; by contrast, UVA hardly causes directly DNA damage and only endogenous or exogenous chromophores through photosensitized reactions can cause DNA damage.

Therefore, photochemical processes leading to DNA lesions can be rationalized into two types of pathways: those involving direct absorption by DNA and those involving photosensitizers. These photoreactions lead to the formation of dimeric pyrimidine photoproducts such as cyclobutane pyrimidine dimers (CPDs), pyrimidine (6-4) pyrimidone photoproducts (6-4PPs) and oxidative lesions such as 8-oxoGuanine (OG), 2,6-diamino-4-hydroxy-5-formamidopyrimidine (FapyG), 5,6-dihydroxy-5,6dihydrothymine ( $\mathrm{Tg})$, 5-hydroxymethyluracil (HmU) and 5-formyluracil (ForU) as shown in Figure 1.2. 


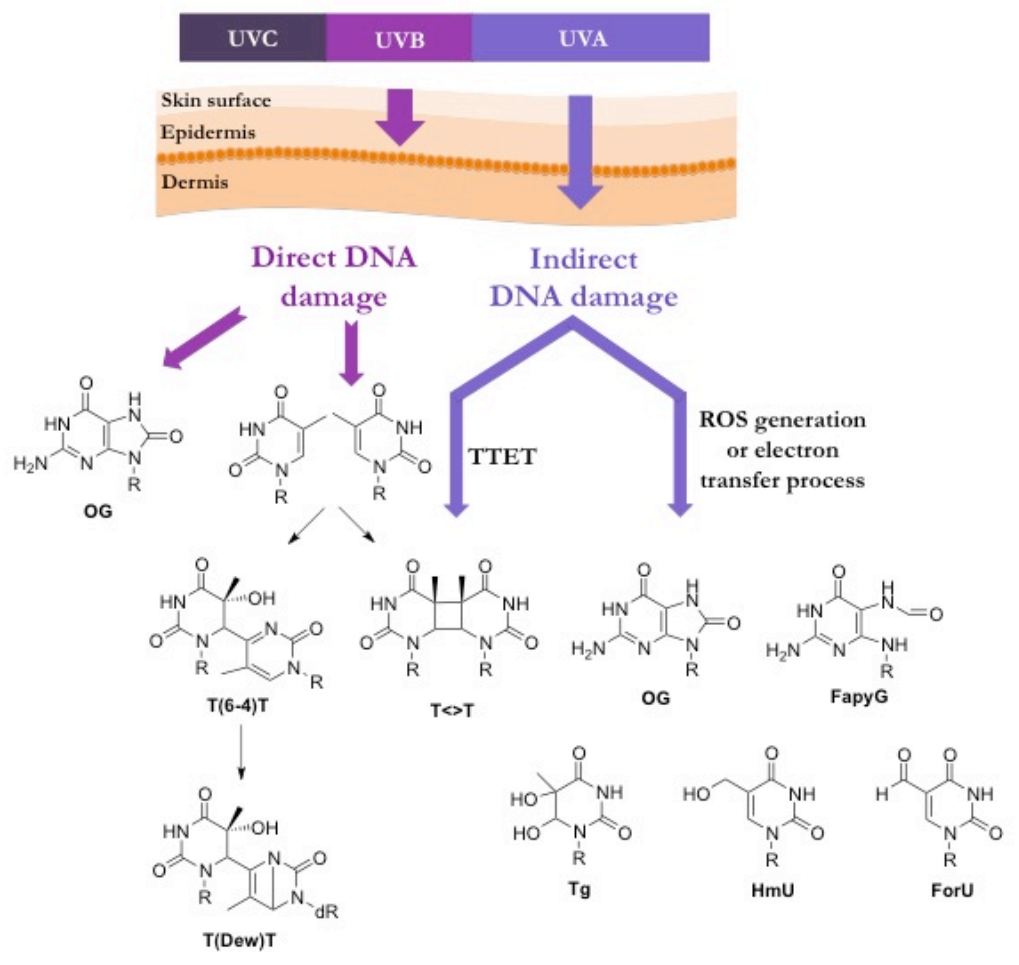

Figure 1.2. DNA lesions induced: (i) by direct absorption of UV (left) and (ii) by photosensitization (right).

\subsubsection{Direct DNA damage}

DNA damage induced by UVC and UVB radiation is dominated by bipyrimidine photoproducts although adenine dimers and pyrimidine photohydrates are formed as well to a much lesser extent. ${ }^{17}$ The formation of oxidation products, more specifically OG (Figure 1.2), is also induced through photoionization in cellular DNA in a lower yield than bipyrimidine photoproducts, which agrees with the fact that guanine exhibits the lowest ionization potential among the DNA bases..$^{18-19}$

UVB is considered the most mutagenic spectral region since it is absorbed directly by the DNA; however, UVA irradiation may also induce CPDs by direct absorption 
but the yield is two or three orders of magnitude lower than for UVB and even larger than for OG in human skin.20-21

Therefore, cis-syn CPDs at TT site $(\mathrm{T}<>\mathrm{T}$ in Figure 1.2) and 6-4PPs $(\mathrm{T}(6-4) \mathrm{T}$ in Figure 1.2) are the main categories of bipyrimidine photoproducts resulting from UVB absorption that causes intranucleotide cross-linking by pyrimidine dimerization. ${ }^{22-23}$ CPDs, the most frequent type of pyrimidine dimers, are formed by $[2+2]$ cycloaddition reaction between the C5-C6 double bonds of two adjacent pyrimidine bases (i.e. TT or TC, Scheme 1.5) and they are obtained in a relatively low quantum yield (ca. $2 \%$ ) in a few picoseconds, as shown by time-resolved measurement in single-stranded DNA. ${ }^{24-25}$ Their formation involves the $\pi \pi^{*}$ excited state; however, the multiplicity of the excited state, $\mathrm{S}$ or $\mathrm{T}$, is still under discussion.

The stereochemistry of the resulting bypirimidine photoproduct is governed by structural features of DNA duplex in B conformation, which is the most common in Nature. Therefore, the predominant CPD isomer is the cis-syn where two adjacent bases show a parallel orientation and are located on the same side with respect to the cyclobutane ring. Besides, CPD formation is influenced by the sequence context ${ }^{26}$ as it is formed exclusively at dipyrimidines and preferentially at TT sites. The efficiency of CPD formation at different dipyrimidine sequences is estimated at a ratio of 55:33:11:1 for 5'-TT > 5'-TC > 5'-CT > 5'-CC. ${ }^{2} 7$

In addition, cytosine containing CPD lesions are particularly mutagenic since they exhibit a deamination reaction by hydrolysis to give the corresponding uracil compounds ( $\mathrm{T}<>\mathrm{U}$ in Scheme 1.5). The original cytosine should lead to the incorporation of guanine upon replication; but uracil would miscode for an adenine like thymine does, giving transition mutations denominated $\mathrm{T} \rightarrow \mathrm{C}$ at $\mathrm{TC}$ sites and the tandem mutation $\mathrm{CC} \rightarrow$ TT. 28 
6-4PPs are produced by a [2+2] Paternò-Büchi cycloaddition between the C5-C6 double bond of the $5^{\prime}$-end pyrimidine and the $\mathrm{C} 4$ carbonyl group of a $3^{\prime}$-end thymine or the C4 imino group of a $3^{\prime}$-end cytosine in a tautomeric form (Scheme 1.5). ${ }^{29}$ This reaction in DNA is proposed to occur via singlet excited state as 6-4PPs are only observed by direct irradiation and not by photosensitized triplet-triplet energy transfer (TTET).

The intermediates of the photoreaction are oxetanes or azetidines $(\mathrm{T}(\mathrm{ox}) \mathrm{T}$ or $\mathrm{T}\left(\right.$ azt) $\mathrm{C}$ in Scheme 1.5), depending on whether thymine or cytosine is the $3^{\prime}$-end base. Both unstable intermediates are converted into their respective 6-4PPs that exhibit a $3^{\prime}$-end pyrimidone ring whose $\mathrm{C} 4$ substituent has migrated to the $\mathrm{C} 5$ position of the pyrimidine moiety. Furthermore, 6-4PPs can be converted to Dewar valence isomers (Dews) by a $4 \pi$ electrocyclization through a singlet excited state intermediate. The formation of these photoproducts results from the presence of the pyrimidone chromophore, which absorbs photons with a maximal efficiency at around $320 \mathrm{~nm}$.

6-4PPs are produced in lower yields than CPDs, being of 1:2 and 1:8 depending on the detection methods and bipyrimidine sequences. ${ }^{30}$ Although 6-4PPs are formed in TT, TC, CT and CC sequences, they occur preferentially at TC sequences in similar yield than CPDs in TC sequences. By contrast, CT and CC sites are much less susceptible to these UVB-photoreactions. 
(a)

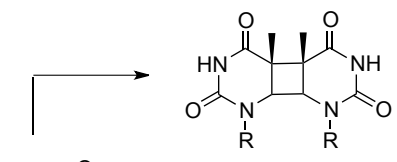<smiles>[R]n1cc(Cc2cn([R])c(=O)[nH]c2=O)c(=O)[nH]c1=O</smiles>

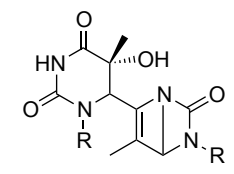

$\mathrm{T}$ (Dew)T
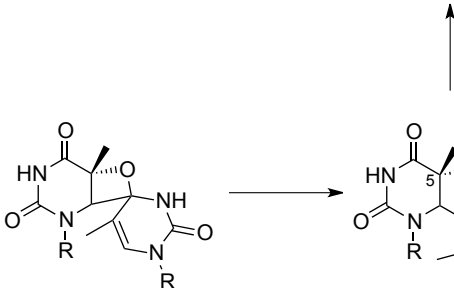
(unstable intermediate)

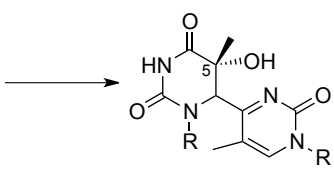

$\mathrm{T}(6-4) \mathrm{T}$

(b)
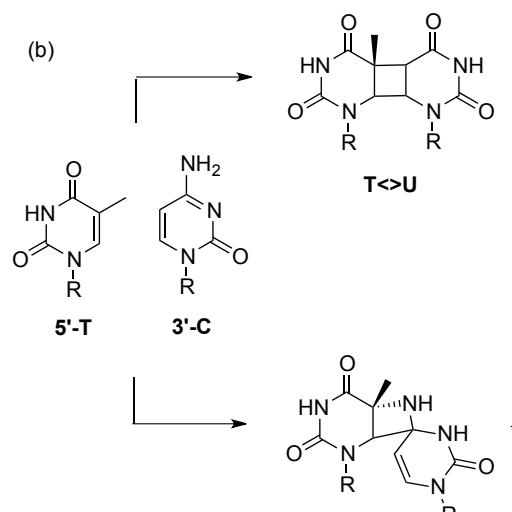

T(azt)C

(unstable intermediate)

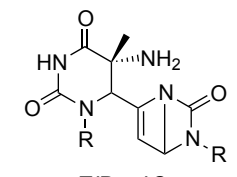

T(Dew)C
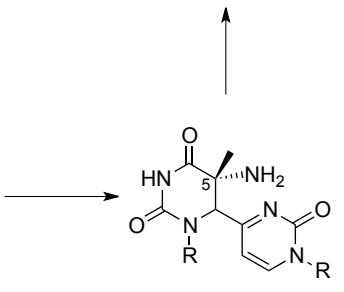

$\mathrm{T}(6-4) \mathrm{C}$

Scheme 1.5. Mechanism of CPDs and 6-4PPs formation for (a) TT and (b) TC sequences.

\subsubsection{Indirect DNA damage}

As mentioned before, DNA hardly absorbs in the UVA range thus most of UVA photoproducts are formed through indirect photosensitized mechanisms after light absorption by endogenous (flavin derivatives, porphyrins, specific vitamins, etc) or exogenous chromophores present in drugs, cosmetic agents, metabolites, etc. These photoreactions are generally induced by the triplet excited state of the photosensitizer $\left({ }^{3} \mathrm{PS}^{*}\right)$ which is longer lived than the singlet excited state. Therefore, three DNA pho- 
tosensitization mechanisms can occur; two of them involve the oxidation of DNA nucleobases by electron transfer and reactive oxygen species (ROS), and the third one involves the formation of CPDs by an energy transfer process (Scheme 1.6).

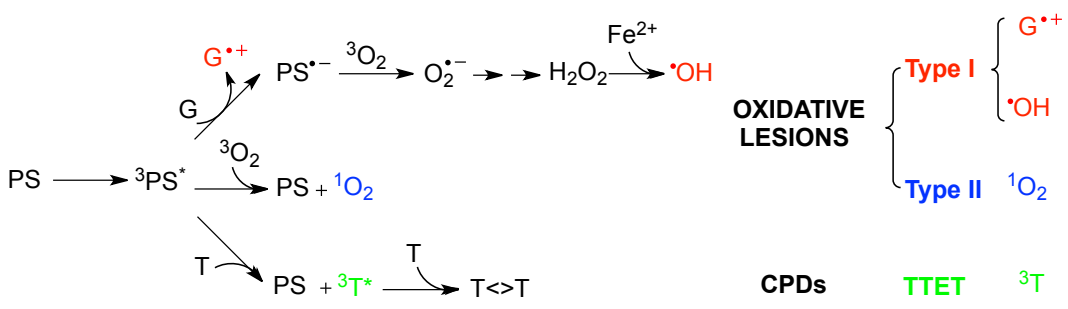

Scheme 1.6. The three different UVA photosensitization mechanisms involved in DNA: (i) one-electron oxidation from PS or addition of ${ }^{\circ} \mathrm{OH}$ (Type I), (ii) addition of singlet oxygen (Type II) and (iii) triplet-triplet energy transfer process (TTET).

Oxidative lesions generally arise from two types of mechanisms, called Type I and Type II processes (Scheme 1.6). ${ }^{31}$ Type I mechanism involves one-electron oxidation by an excited chromophore, which generally leads to the formation of a radical cation of the DNA base and a radical anion of the photosensitizer (Scheme 1.6). Thus, the efficiency of this photoreaction is forced by the oxidation potential of the photosensitizer. In DNA, guanine is the main target as it exhibits the lowest ionization potential among DNA nucleobases. After an electron abstraction, guanine is converted into a radical cation $\left(\mathrm{G}^{\bullet+}\right)$ that may evolve into several products. The main pathway is hydration into the 8-hydroxy-7,8-dihydroguanyl radical (Scheme 1.7), which can be converted to $O G$ and FapyG by a competitive one-electron oxidation or one-electron reduction, respectively. 32

Type I photosensitization mechanism may also involve the formation of the highly reactive hydroxyl radicals $\left({ }^{\circ} \mathrm{OH}\right)$, see Scheme 1.6.29 They are obtained after release of superoxide anion $\mathrm{O}_{2}{ }^{\bullet-}$, which comes from the reaction of the photosensitizer radical anion with molecular oxygen or by release from mitochondria in response to UVA irradiation..$^{33}$ Since $\mathrm{O}_{2}{ }^{\bullet-}$ is poorly reactive with DNA, it can be converted by 
spontaneous reaction or by superoxide dismutase enzyme into hydrogen peroxide $\left(\mathrm{H}_{2} \mathrm{O}_{2}\right)$, followed by a redox process with $\mathrm{Fe}^{2+}$ or $\mathrm{Cu}^{+}$, known as Fenton reaction, to give ${ }^{\bullet} \mathrm{OH}$. UVA has also been shown to release $\mathrm{Fe}^{2+}$ from ferritin that favors the Fenton reaction. ${ }^{34}$

By contrast to one-electron oxidation, ${ }^{\bullet} \mathrm{OH}$ does not show any specificity and reacts with all the components of DNA..$^{35}$ Therefore, the reaction of ${ }^{\bullet} \mathrm{OH}$ with purine bases leads to OG and FapyG for guanine (Figure 1.2) and 8-oxo-7,8-dihydroadenine and 4,6-diamino-5-formamidopyrimidine for adenine through formation of C8hydroxylated radicals (Scheme 1.7). Regarding to pyrimidine bases, ${ }^{\bullet} \mathrm{OH}$ reacts with the C5-C6 double bond, leading to the formation of $\mathrm{Tg}$ and 5,6-dihydroxy-5,6dihydrocytosine or with the methyl group of thymine leading to $\mathrm{HmU}$ and ForU (Figure 1.2). Besides, ${ }^{\bullet} \mathrm{OH}$ reacts with the 2 -deoxyribose moieties by hydrogen abstraction leading to DNA strand breaks. ${ }^{36}$

A second oxidative pathway, denominated Type II, involves the production of singlet oxygen $\left({ }^{1} \mathrm{O}_{2}\right)$ that results from energy transfer from the triplet excited photosensitizer to molecular oxygen (Scheme 1.6). These reactive species show strong affinity for molecules rich in double bonds, giving rise to dioxetanes, endoperoxides or ene-oxidation products. In DNA, guanine is the only target, that is converted into a 4,8-endoperoxide through Diels Alder [4+2] photocycloaddition reaction, followed by a rearrangement and reduction to give OG (Scheme 1.7).37 


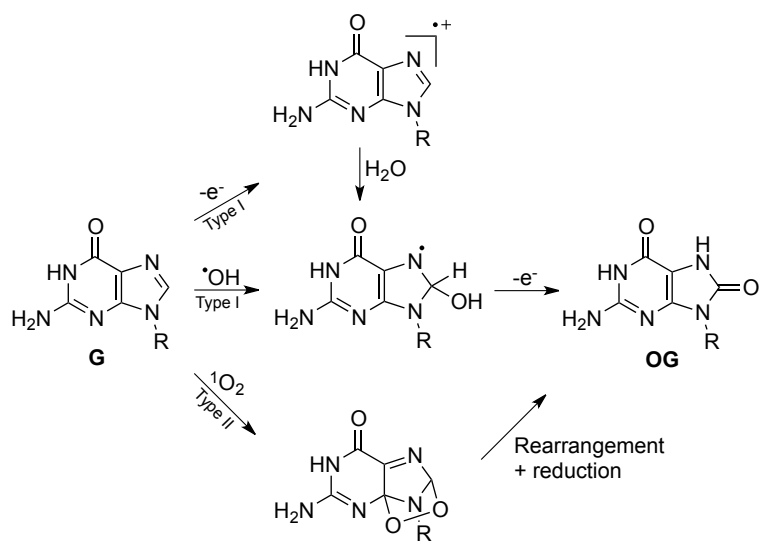

Scheme 1.7. The main mechanisms leading to the formation of OG from G. From top to bottom: (i) electron abstraction followed by hydration of the resulting radical cation, (ii) formation of a reducing radical by addition of ${ }^{\bullet} \mathrm{OH}$ radical, and (iii) formation of an endoperoxide by addition of singlet oxygen.

In contrast to the abovementioned UVA photosensitized mechanisms leading to oxidative lesions, the third one involves the formation of CPDs through triplet-triplet energy transfer (TTET, Scheme 1.6). ${ }^{8}$ This photoreaction requires an endogenous or exogenous chromophore that efficiently absorbs UVA, that has a high energy of triplet state and a large ISC quantum yield to convert the singlet into the triplet excited state. Under these conditions, a TTET can take place from the excited photosensitizer to a nearby DNA base, being thymine the main target because of its low-lying triplet excited state, which leads to the formation of CPDs. ${ }^{38}$ Exogenous chromophores such as anti-inflammatory compounds or antibacterial agents of the fluoroquinolone family are potent TTET photosensitizers and have an important impact in human health. ${ }^{39-40}$ Recently, our group reported that 6-4PPs and ForU lesions are able to act as DNA endogenous sensitizers. It was found that pyrimidone chromophore in 6-4PP lesion and ForU absorb in UVA, and that the high energy of their triplet and high ISC, allow phototriggering the $[2+2]$ cycloaddition between two adjacent thymines. ${ }^{41-42}$ 


\subsection{DNA repair}

When they are not repaired correctly, the UV lesions inhibit DNA replication and transcription, leading to cell death or mutagenesis and carcinogenesis. ${ }^{14-15}$ To maintain their genetic integrity, organisms have developed various DNA repair mechanisms, which can be divided into two general classes: (1) the dark excision pathway such as nucleotide excision repair ${ }^{43}$ or base excision repair ${ }^{44}$ that removes the damaged bases and replaces them with newly synthesized DNA and (2) light triggered repair that is catalyzed by photoactivated enzymes called DNA photolyases..$^{45}$

\subsubsection{Nucleotide excision repair}

Nucleotide excision repair (NER) removes a wide range of structurally unrelated DNA lesions that signiticantly modify the tridimensional structure of DNA double helix such as CPDs and 6-4PPs, bulky chemical adducts, DNA-intrastrand crosslinks, and some oxidative lesions. NER operates through a "cut-and-patch" mechanism by excising and removing a short stretch of DNA (24 to 32 nucleotides long) containing the lesion, which is restored using the nondamaged strand of the DNA double helix as a template (Figure 1.3). Then, the resulting gap is filled by DNA polymerases and sealed by a DNA ligase enzyme. Two pathways (TCR ${ }^{46}$ or GGR ${ }^{47}$ ) depending on the DNA damage signalling step are involved, being TCR much faster than GGR. However, TCR is limited to the lesions present in the transcribed strand of active genes and GGR removes lesions in the whole genome with a lower efficiency. For bypirimidine lesions, GGR removes 6-4PPs and Dews faster than CPDs because of significant perturbations induced in the duplex by the cyclobutane structure. ${ }^{48-49}$ In cultured cells, 6-4PPs are typically removed within a few hours while CPDs can be found three days after exposure, being the repair rate even slower in skin. Moreover, the four CPDs are repaired at different rates: $\mathrm{CT}<\mathrm{CC}<\mathrm{TC}<\mathrm{TT} .{ }^{50}$ 


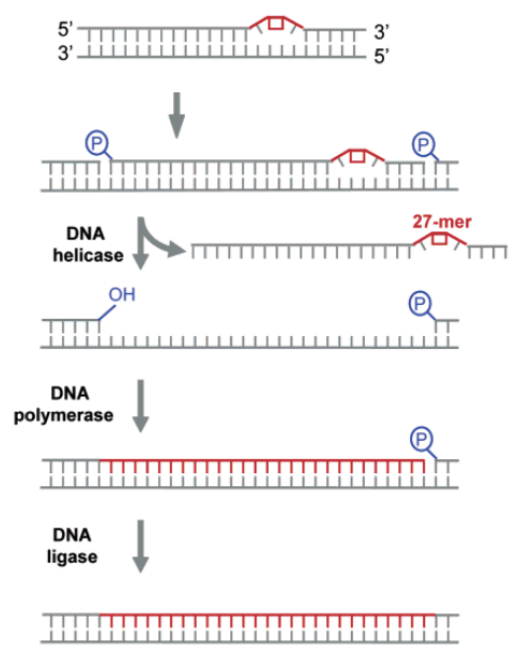

Figure 1.3. Repair mechanism of UV photoproducts by nucleotide excision repair.

\subsubsection{Base excision repair}

Base excision repair (BER) removes small DNA modifications such as oxidized or methylated bases through $\mathrm{N}$-glycosylases action. ${ }^{51}$ These enzymes are substratespecific and each recognizes a limited number of modified bases such as OG for OGG1 or oxidized pyrimidines for NTHL1 and NEIL1 glycosylases. After binding to the modified bases, glycosylases cleave the $\mathrm{N}$-glycosidic bond, leaving an abasic site in DNA. This is then cleaved by an endonuclease, in particular APE1, leaving a onenucleotide gap with a $3^{\prime}$-end $\mathrm{OH}$ and a $5^{\prime}$-end ribosyl-phosphate. The resulting singlestrand break can be repaired by a short-patch (where a single nucleotide is replaced) or long-patch BER (where 2-10 new nucleotides are synthesized) using the complementary strand as a template. By contrast with NER, BER is a fast system, which repairs single-strands breaks in less than one hour and OG in a few hours after exposure to UVA. ${ }^{52}$ 


\subsubsection{Photoreactivation}

By contrast to NER, photoreactivation repairs bypirimidine lesions by means of a specific class of flavoprotein and photoenzyme called photolyase that uses sunlight as energy source and only involves a protein to recognize and repair the lesion. Two types of photolyase with similar sequences and folding structure specifically repair the two bypirimidine photoproducts upon the absorption of blue light. They are classified as CPD or as (6-4) photolyases. ${ }^{45}, 53$ CPD photolyases have been found in diverse groups such as archaea, bacteria, fungi, viruses, plants, invertebrates, and many vertebrates including aplacental mammals, whereas, (6-4) photolyases have been determined in certain organisms like Drosopbila, silkworm, Xenopus laevis, and rattlesnakes. However, photolyases are absent in humans and other placental mammals although they keep homologs flavoproteins denominated cryptochromes, which are involved in various blue light-regulated functions, but typically do not repair DNA.54-55

Therefore, CPD or (6-4) photolyases are homologous monomeric proteins of 450-550 amino acids that contain a non-covalently bound flavin adenine dinucleotide (FAD) chromophore, which can be found in three redox states denominated fully oxidized $\left(\mathrm{FAD}_{\mathrm{ox}}\right)$, semi-reduced $\left(\mathrm{FADH}^{\bullet}\right)$ and fully reduced $\left(\mathrm{FADH}^{-}\right.$, Figure 1.4) forms. Among them, the fully reduced $\mathrm{FADH}^{-}$, which absorbs $350-500 \mathrm{~nm}$ light, is the repair-active form, which is essential for specifically binding to damaged DNA and for catalysis. ${ }^{56} \mathrm{FAD}_{\mathrm{ox}}$ and $\mathrm{FADH}^{\bullet}$ can be converted to $\mathrm{FADH}^{-}$through intraprotein electron transfer (ET) photoreaction called photoactivation that involves a chain of three tryptophan residues. ${ }^{57}$ Moreover, photolyases present a second chromophore, 8hydroxy-5-deazaflavin (8-HDF, Figure 1.4) or methenyltetrahydrofolate (MTHF, Figure 1.4), which acts as a light-harvesting antenna with a higher extinction coefficient than $\mathrm{FADH}^{-}$thereby increasing the effective absorption cross section of the enzyme by a transfer of excitation energy to the catalytic FAD cofactor. ${ }^{58}$ 


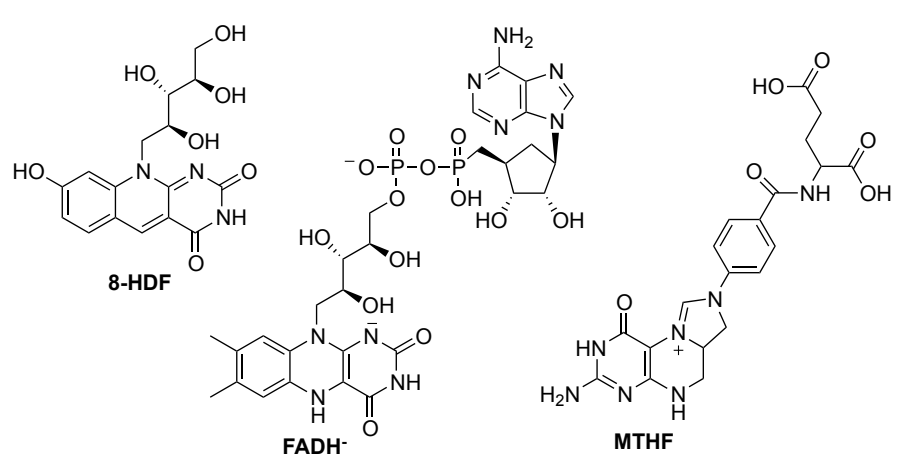

Figure 1.4. The structure of the non-covalently bound chromophores of a photolyase.

The photorepair mechanism by CPD photolyase has been fully established. The initial step consists in the absorption of a photon by the chromophore 8-HDF or MTHF, followed by energy transfer to the $\mathrm{FADH}^{-}$chromophore. Then, the photoexcited $\mathrm{FADH}^{-}$donates an electron to the $\mathrm{CPD}$, inducing ring splitting of the C-C bonds to restore the two intact pyrimidines. By contrast of CPD photolyase mechanism, repair of 6-4PPs is chemically more challenging because cleavage of the $\mathrm{C}-\mathrm{C}$ bond between the 5' and 3' pyrimidine nucleobases alone would yield two damaged nucleobases, thus an additional back transfer of a functional group $\left(\mathrm{OH}\right.$ or $\left.\mathrm{NH}_{2}\right)$ from the 5' to the 3' nucleobase must take place.

\subsubsection{CPD photolyase mechanism}

As mentioned before, CPD photolyase mechanism has been established and all steps have been described by means of ultrafast time-resolved spectroscopies. ${ }^{59-62}$ The photoreaction may be summarized as follows (Scheme 1.8). Prior to repair, CPD photolyase binds to DNA and flips the CPD lesion out of the DNA duplex ${ }^{63}$ into the active site cavity where FAD is buried to make a stable enzyme-substrate complex and ensure an efficient ET between CPD and the FAD cofactor. After photoexcitation of FAD in its fully reduced $\mathrm{FADH}^{-}$form, an electron is tranferred to the CPD in $250 \mathrm{ps}$, 
leading to the semireduced radical $\mathrm{FADH}^{\bullet}$ and to the radical anion of the $\mathrm{CPD}$, which rapidly splits in 90 ps and rearranges into one intact base and the radical anion of the other base. After the dimer splitting, an electron is transferred back to FADH ${ }^{\bullet}$ to regenerate the second base and the catalytically form $\mathrm{FADH}^{-}$in 700 ps. ${ }^{64}$ Then, the CPD photolyase releases the repaired bypirimidine lesion from the binding pocket in $\sim 50 \mu \mathrm{s}^{65}$, and can bind and repair another CPD.

The photorepair mechanism is a monophotonic process with a high quantum yield of 0.7-0.9,45 which suggests non-negligible competition between CPD splitting (90 ps) and back electron transfer from $\mathrm{FADH}^{\bullet}$ to $\mathrm{FADH}^{-}$, which happens on a nanosecond timescale $(2.4 \mathrm{~ns})$. Although the CPD repair mechanism has been determined, the exact quantum yield and the rate of unproductive back electron transfer (2.4 ns or $350 \mathrm{ps}$ ) are still under discussion.66-67

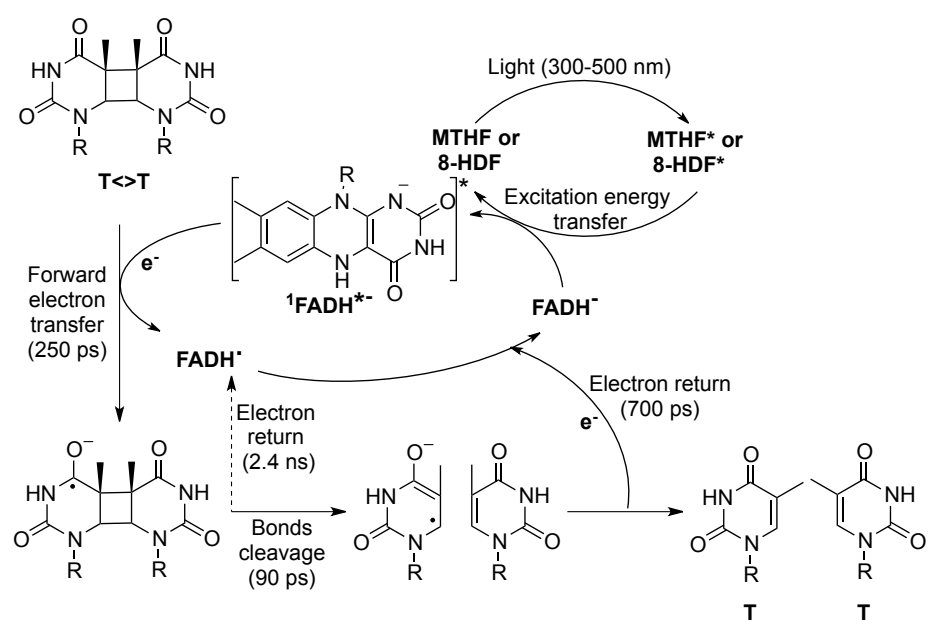

Scheme 1.8. Photorepair mechanism of CPD lesions by CPD photolyases. 


\subsubsection{2 (6-4) photolyase mechanism}

Two main reasons attracted the interest for the repair of 6-4PPs. First, (6-4) photolyases restore (6-4) photolesions but with a lower quantum yield of repair of ca. 0.1 compared to that of CPD lesion of ca. 0.7-0.9.45 Secondly, whereas the two-step retro$[2+2]$ cycloaddition reaction of the CPD lesion leads to the repaired nucleobases, the restoration of (6-4) lesions is structurally and chemically more challenging, as it requires an $\mathrm{OH}$ or $\mathrm{NH}_{2}$ group transfer from 5' to the 3' nucleobase. By contrast with the CPD photolyase mechanism, researchers are still addressing the question of how (6-4) photolyases perform such return of the $\mathrm{OH}$ or $\mathrm{NH}_{2}$ group, and several mechanisms have been proposed until now.

\section{Thermal oxetane mechanism}

The strong structural similarity between (6-4) and CPD photolyases suggests an analogy in the photorepair mechanisms for (6-4) and CPD lesions. Therefore, it was initially proposed, that upon binding to the lesion, (6-4) photolyases first catalyze the formation of a four-membered ring intermediate (oxetane or azetidine), similar to the cyclobutane for CPDs, by a thermal reaction in the dark (Scheme 1.9).45 Then, the splitting of this intermediate to the repaired pyrimidine bases would follow just as for CPD photolyases, by ET from the excited fully reduced $\mathrm{FADH}^{-}$to the lesion.

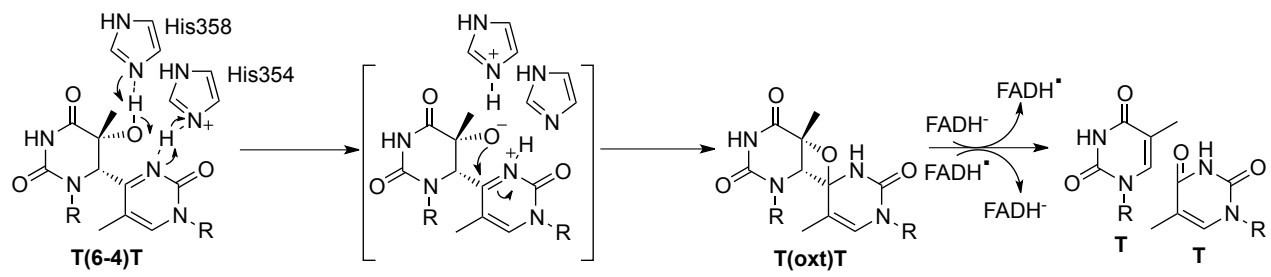

Scheme 1.9. First repair mechanism proposed for 6-4PP. 
Although theoretical studies indicate that the formation of oxetane intermediate is energetically rather unfavorable for an enzyme, ${ }^{68}$ the identification of two histidines (His) residues (His358 and His354) in the binding pocket, which are crucial for catalysis, would provide sufficient energy to close the four-membered ring intermediate by proton transfer. ${ }^{69}$ The His residue may protonate the (6-4) photoproduct, or at least form a strong $\mathrm{H}$-bond with the $\mathrm{N} 3$ of the 3' residue to generate a highly electrophilic iminium ion, which can be attacked by the $\mathrm{OH}$ or $\mathrm{NH}_{2}$ group to form the oxetane or azetidine intermediate.

\section{Non-oxetane mechanism}

In 2008, a new photorepair mechanism was postulated based on the crystal structures of Drosophila melanogaster (6-4) photolyase bound to a 15-mer DNA duplex containing a central $\mathrm{T}(6-4) \mathrm{T}$ lesion before and after in situ repair. ${ }^{70-71}$ The crystal structure before repair revealed that the lesion was flipped out of the double helix into the binding pocket of the enzyme, in a similar way to the CPD case, together with the conservation of histidines His365 and His369, which are essential for catalysis, and of a tyrosine residue (Y423). Unexpectedly, oxetane intermediate was not catalyzed in the dark by these amino acids through the abovementioned proton transfer since the lesion showed its original T(6-4)T structure. Moreover, the crystal of the enzyme-substrate after repair, using white light and dithionite as reducing agent, showed a repaired T-T in the active site with almost no change in the position of the amino residues located in the close vicinity to the lesion. ${ }^{70}$ Therefore, the structural data of the complex enzyme-substrate before and after in situ repair does not support the oxetane-based mechanism assumed previously and a direct ET from $\mathrm{FADH}^{-}$to the 6-4PP was proposed, see Scheme 1.10.

Therefore, after ET from a photoexcited $\mathrm{FADH}^{-}$, a radical anion of the 6-4PP is formed and a $\mathrm{C} 5-\mathrm{OH}$ or a $\mathrm{C} 5-\mathrm{NH}_{2}$ is converted into a better leaving group by His 365 through protonation. After that, the protonated transient $\mathrm{NH}_{3}$ group or water mole- 
cule released in $\mathrm{C} 5$ attacks the acylimine in $\mathrm{C} 4$ ' and it rapidly fragments to give the repaired T-T, accompanied by back electron transfer to $\mathrm{FADH}^{\bullet}$ and loss of a proton. However, this "transient water pathway" was not supported by computational studies because of a high activation barrier, but variants of this mechanism are still under discussion..$^{72}$

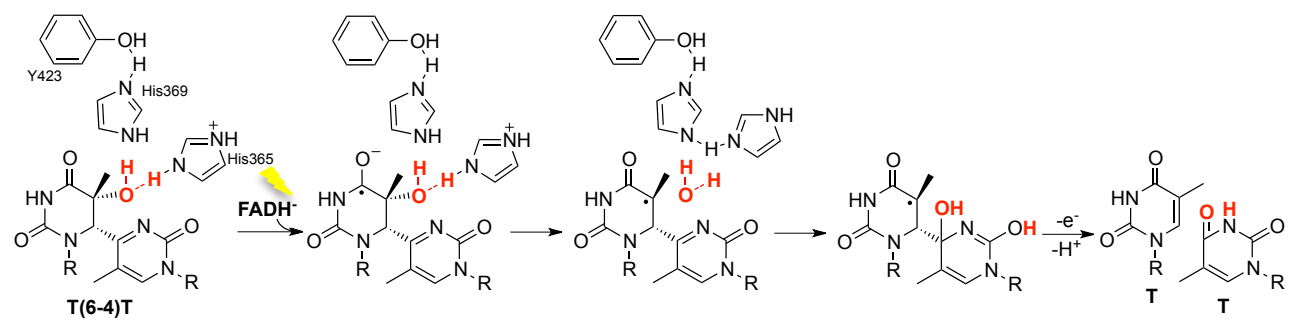

Scheme 1.10. First non-oxetane repair mechanism of 6-4PP by (6-4) photolyase. This pathway involves the formation of a transient water molecule, indicated in red.

In 2010, a modified mechanism by the (6-4) photolyases of Arabidopsis thaliana was postulated. This time, ultrafast fluorescence and transient absorption spectroscopy supports the formation of an oxetane or azetidine structure as short-lived species. ${ }^{73}$ In this alternative mechanism, the main steps are the initial electron transfer from the excited reduced $\mathrm{FADH}^{-}$to the 6-4PP in $225 \mathrm{ps}$ (similar to the photorepair reaction of CPD $)^{64}$ and the following proton transfer from protonated His 364 in 425 ps. A transient zwitterion is generated where an oxygen-atom can attack the C4 position at the $3^{\prime}$ base to form a transient oxetane structure, which rearranges to the repaired pyrimidine bases with electron return to $\mathrm{FADH}^{\bullet}$, see Scheme 1.11. This last step takes place on a timescale of tens nanoseconds, it is much longer than for CPD case where repair is completed by electron return to $\mathrm{FADH}^{\bullet}$ in 700 ps.

In addition, $\mathrm{FADH}^{\bullet}$ radicals formed by the initial ET were re-reduced very quickly (in 50 ps) by back ET by contrast to CPD (in $2.4 \mathrm{~ns}$ ). These findings revealed that the reason for the low repair quantum yield of (6-4) photolyase of ca. 0.1 is the 
fast back electron transfer compared to the proton transfer step (of ca. 425 ps), which suggests that it is the critical step in the (6-4) lesion repair mechanism.

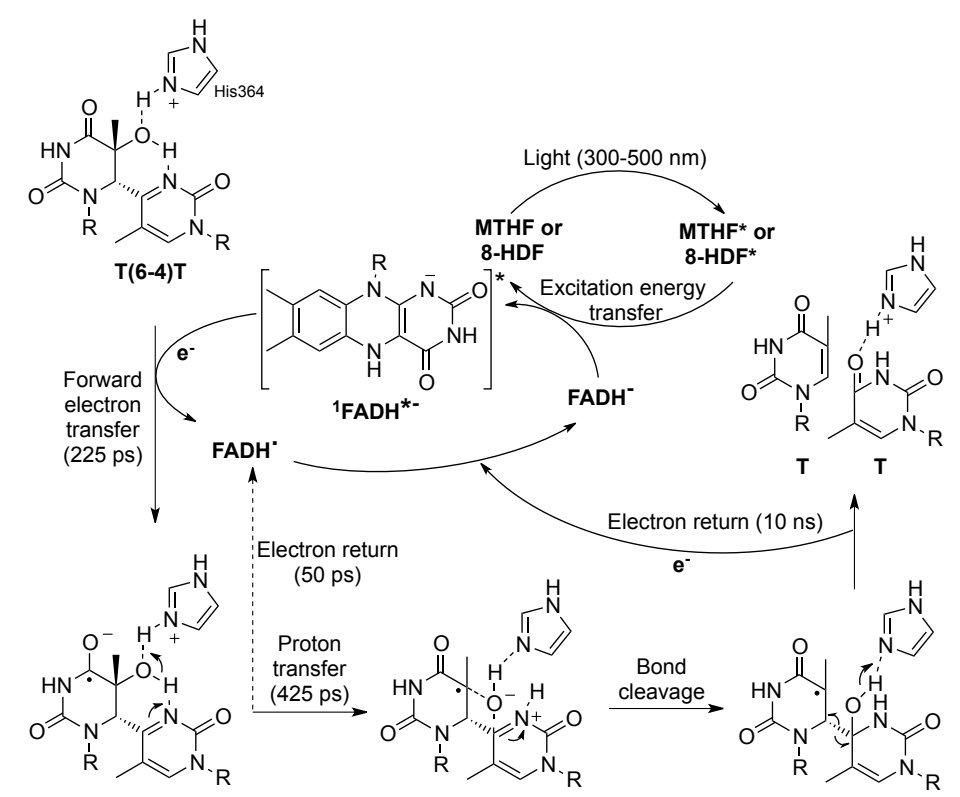

Scheme 1.11. Repair photocycle of (6-4)PP by (6-4) photolyase of Arabidopsis thaliana. ${ }^{73}$

\section{Two photon mechanism}

Theoretical and experimental studies assumed that the (6-4) and CPD photolyase mechanisms are a monophotonic process where FAD cofactor absorbs only a single photon. However, the possibility of a two-photon process has been reported by a computational study in $2010 .{ }^{74}$ They suggested that a first ET from $\mathrm{FADH}^{-}$converts $\mathrm{T}(6-4) \mathrm{T}$ to the oxetane; followed by a second $\mathrm{ET}$ from $\mathrm{FADH}^{-}$to split the oxetane ring into the two intact thymines with the return of the electron.

In this context, in 2013 an experimental two-photon process was proposed to the repair of a $\mathrm{T}(6-4) \mathrm{T}$ by Xenopus laevis (6-4) photolyase under single turnover flash experiments. ${ }^{75}$ They observed that a first ET converts $\mathrm{T}(6-4) \mathrm{T}$ into an intermediate $\mathrm{X}$, 
which absorbs at shorter wavelengths than the $\mathrm{T}(6-4) \mathrm{T}$, and with a lifetime long enough (100 s) to allow the absorption of a second photon that converts $\mathrm{X}$ to the final repaired product (Scheme 1.12). Interestingly, the quantum yield that they obtained for the conversion of $\mathrm{X}$ to intact thymines was $0.5-1$, which is similar to the repair quantum yield of CPD photolyase. This analogy of cycloreversion of the fourmembered ring by CPD and (6-4) photolyases made them suggest that X might be the oxetane ring intermediate. Therefore, a new mechanism that involves an oxetane ring intermediate was proposed although a positive proof of this intermediate is still lacking.

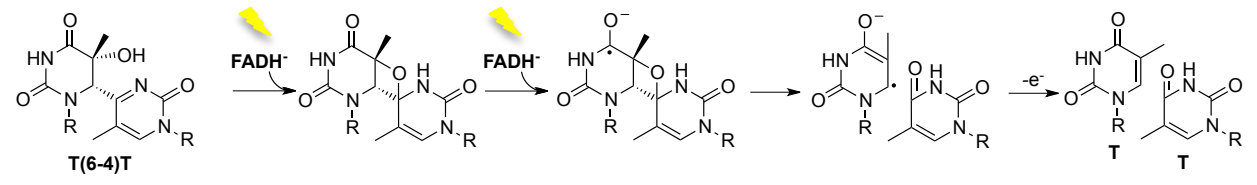

Scheme 1.12. First experimental repair mechanism of (6-4)PP by (6-4) photolyase through two photon process.

\subsection{Investigation of the (6-4) repair mechanism with model compounds}

As mentioned before, photorepair of (6-4) DNA lesions by photolyases involved a photoinduced electron transfer (PET) from FAD cofactor to the bipyrimidine lesion; however, a critical issue is whether (photo)chemical conversion of the 6-4PPs to oxetanes or azetidines is a necessary step. Besides, calculations at various levels of theory ${ }^{76}$ questioned the ability of the (6-4) photolyase to form the oxetane and azetidine intermediates during the repair reaction because the enormous energy necessary to run the reaction is too high for an enzyme. Unfortunately, the oxetane or azetidine intermediate involved in the proposed mechanism does not seem to be stable above $80^{\circ} \mathrm{C}$, making their characterization difficult. ${ }^{77}$ 
In this context, model systems have been developed to gain insight into the ET and ring opening of these four-member ring heterocycles. Stable oxetane adducts (ox, Figure 1.5), formed between benzophenone and thymine by Pàterno-Büchi reaction, have been reported to reverse by PET in the presence of various electron donors, which makes the theory of oxetane intermediate chemically feasible..$^{78}$ Moreover, laser flash photolysis and steady-state studies with thymine oxetane adducts provided additional support for the facile cleavage of the oxetane anionic radical. ${ }^{78-80}$ The strongest support came from the investigation of photoinduced cycloreversion of an oxetane ring covalently linked to flavin (ox-flav, Figure 1.5). ${ }^{81}$ In this model system, it was found that the cycloreversion is only possible through the reductive pathway with the flavin in its reduced and deprotonated state.

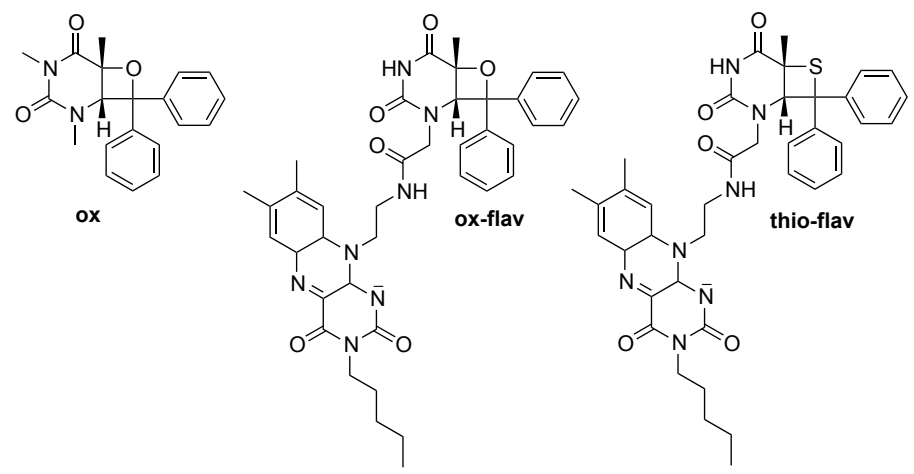

Figure 1.5. Oxetane and thiethane models studied by PET.

In contrast to the oxetane or azetidine intermediate, its sulfur analog, a thietane, formed by $360 \mathrm{~nm}$ irradiation of a dinucleotide 5'-O-thymidylyl-4-thiothymidine, was reported to be moderately stable and to interconvert with the corresponding $\mathrm{s}^{5}-(6-$ 4) $\mathrm{PP}$ at room temperature (Scheme 1.13).82 


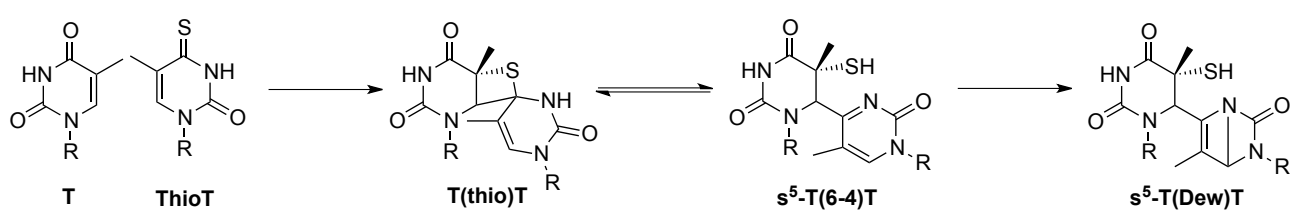

Scheme 1.13. Mechanism formation of thio analog of 6-4PP.

Longer irradiation time led to the total conversion of compound $\mathrm{s}^{5}-\mathrm{T}(6-4) \mathrm{T}$ into its Dewar valence isomer s $\mathrm{s}^{5} \mathrm{~T}$ (Dew) $\mathrm{T}$. Indeed, $\mathrm{T}$ (thio) $\mathrm{T}$ and $\mathrm{s}^{5}-\mathrm{T}(6-4) \mathrm{T}$ consisted of a mixture of two slowly interconverting compounds in a $3 / 1$ ratio. The thietane structure was evidenced by ${ }^{1} \mathrm{H}$ NMR since the chemical shift of the H6 proton of the T unit of $\mathrm{T}$ (thio) $\mathrm{T}$ is at $6.30 \mathrm{ppm}$, which is very similar to the value $(6.10 \mathrm{ppm})$ of the H6 proton of a model thietane obtained by irradiation of 1,3-dimethyl-4-thiothymine in the presence of methacrylonitrile. ${ }^{83}$

In order to investigate if the enzymatic repair reaction with (6-4) photolyases could occur, a stable thiethane intermediate $\mathrm{T}$ (thio) $\mathrm{T}$ was inserted in an oligonucleotide $\mathrm{d}$ (GTATs ${ }^{4}$ TATG). It was shown that $\mathrm{T}$ (thio) $\mathrm{T}$ is not enzymatically repaired by Drosophila (6-4) photolyases, this can be attributed to inefficient binding rather than to lack of ET reactivity. ${ }^{84}$ The lack of binding might also be due to substantial structural differences that result from differences in the lengths of C-S and either C-O or C-N bonds (1.8 versus $1.5 \AA$ ). However, the methyl s ${ }^{5}-\mathrm{T}(6-4) \mathrm{T}$, which presumably cannot form the thiethane intermediate, bound the enzyme with high affinity and it was repaired sucessfully.

In this context, to support that thietanes could be cleaved by single electron donation, the cycloreversion of a stable thiethane (thio-flav, Figure 1.5), formed between thymine and thiobenzophenone and covalently linked flavin moiety, has been achieved by intramolecular PET. 85 As shown for the oxetane case, the cycloreversion required the flavin in its reduced and deprotonated state. 


\subsection{References}

1. Jablonski, A. Z., Über den mechanismus der photolumineszenz von farbstoffphosphoren. Phys. 1935, 94, 38-46.

2. Speiser, S., Photophysics and mechanisms of intramolecular electronic energy transfer in bichromophoric molecular systems: solution and supersonic jet studies. Chem. Rev. 1996, 96, 1953-1976.

3. Kavarnos, G. J.; Turro, N. J., Photosensitization by reversible electron transfer: theories, experimental evidence and examples. Chem. Rev. 1986, 86, 401-449.

4. Weller, A., Photoinduced electron transfer in solution: exciplex and radical ion pair formation free enthalpies and their solvent dependence. Phys. Chem. Neue Folge. 1982, 133, 93-98.

5. Griesbeck, A.; Oelgemöller, M.; Ghetti, F., CRC Handbook of Organic Photochemistry and Photobiology. 3rd Ed. 2012, 95-118.

6. Bach, T., Stereoselective intermolecular $[2+2]$ photocycloaddition reactions and their application in synthesis. Synthesis 1998, 5, 683-703.

7. Schuster, D. I.; Lem, G.; Kaprinidis, N. A., New insights into an old mechanism: [2+2] photocycloaddition of enones to alkenes. Chem. Rev. 1993, 93, 3-22.

8. Cuquerella, M. C.; Lhiaubet-Vallet, V.; Bosca, F.; Miranda, M. A., Photosensitised pyrimidine dimerisation in DNA. Chem. Sci. 2011, 2, 1219-1232.

9. Buchi, G.; Inman, C. G.; Lipinsky, E. S., Light-catalyzed organic reactions. I. The reaction of carbonyl compounds with 2-methyl-2-butene in the presence of ultraviolet light. J. Am. Chem. Soc. 1954, 4327-4331.

10. Paterno, E., Organic synthesis induced by light. Introductory note. Gaz: Chim. Ital. 1909, 39, 237-250.

11. Freilich, S. C.; Peters, K. S., Observation of the 1,4 biradical in the PaternoBuchi reaction. J. Am. Chem. Soc. 1981, 103, 6255-6257.

12. Sampedro, D., Computational exploration of the photocycloaddition of imines to alkenes. ChemPbysChem 2006, 7, 2456-2459. 
13. McKenzie, R. L.; Aucamp, P. J.; Bais, A. F.; Björn, L. O.; Ilyas, M.; Madronich, S., Ozone depletion and climate change: impacts on UV radiation. Photochem. Photobiol. 2011, 10, 182-198.

14. Melnikova, V. O.; Ananthaswamy, H. N., Cellular and molecular events leading to the development of skin cancer. Mutat. Res. 2005, 571, 91-106.

15. Coohill, T. P.; Peak, M. J., The effects of the ultraviolet wavelengths of radiation present in sunlight on human cells in vitro. Photochem. Photobiol. 1987, 46, 1043-1050.

16. Schuch, A. P.; Machado, C. C.; Makita, K.; Martins, C. F., DNA damage as a biological sensor for environmental sunlight. Photochem. Photobiol. 2013, 12, 1259-1272.

17. Cadet, J.; Sage, E.; Douki, T., Ultraviolet radiation-mediated damage to cellular DNA. Mutat. Res. 2005, 571, 3-17.

18. Kielbassa, C.; Roza, L.; Epe, B., Wavelength dependence of oxidative DNA damage induced by UV and visible light. Carcinogenesis 1997, 18, 811-816.

19. Gomez, M.; Banyasz, A.; Douki, T.; Markovitsi, D.; Ravanat, J., Direct oxidative damage of naked DNA generates upon absorption of UV radiation by nucleobases. J. Phys. Chem. Lett. 2016, 7, 3945-3948.

20. Mouret, S.; Baudouin, C.; Charveron, M.; Favier, A.; Cadet, J.; Douki, T., Cyclobutane pyrimidine dimers are predominant DNA lesions in whole human skin exposed to UVA radiation. Proc. Natl. Acad. Sci. 2006, 103, 13765-13770.

21. Mouret, S.; Philippe, C.; Chantegrel, J.; Banyasz, A.; Karpati, S.; Markovitsi, D.; Douki, T., UVA-induced cyclobutane pyrimidine dimers in DNA: a direct photochemical mechanism? Org. Biomol. Chem. 2010, 8, 1706-1711.

22. Pfeiter, G. P., Formation and processing of UV photoproducts: effects of DNA sequence and chromatin environment. Photochem. Photobiol. 1997, 62, 270-283.

23. Yoon, J. H.; Lee, C. S.; O’Connor, T. R.; Yasui, A.; Pfeifer, G. P., The DNA damage spectrum produced by simulated sunlight. J. Mol. Biol. 2000, 299, 681-693. 
24. Garcés, F.; Dávila, C. A., Alteration in DNA irradiated with ultraviolet radiation. The formation process of cyclobutylpyrimidine dimers: cross sections, action spectra and quantum yields. Photochem. Photobiol. 1982, 35, 9-16.

25. Schereier, W. J.; Schrader, T. E.; Koller, F. O.; Gilch, P.; Crespo-Hernández, C. E.; Swaminathan, V. N.; Carell, T.; Zinth, W.; Kohler, B., Thymine dimerization in DNA is a ultrafast photoreaction. Science 2007, 315, 625-629.

26. Tornaletti, S. R. D.; Pfeifer, G. P., The distribution of UV photoproducts along the human p53 gene and its relation to mutation in skin cancer. Oncogene 1993, 8, 2051-2057.

27. Douki T, C. J., Individual determination of the yield of the main UV-induced dimeric pyrimidine photoproducts in DNA suggests a high mutagenicity of CC photolesions. Biochemistry 2001, 40, 2495-2501.

28. Peng, W.; Shaw, B. R., Accelerated deamination of cytosine residues in UVinduced cyclobutane pyrimidine dimers leads to CC to T'T transitions. Biochemistry 1996, 35, 10172-10181.

29. Cadet, J.; Mouret, S.; Ravanat, J.; Douki, T., Photoinduced damage to cellular DNA: direct and photosensitized reactions. Photochem. Photobiol. 2012, 88, 1048-1065.

30. Ravanat, J. L.; Douki, T.; Cadet, J., Direct and indirect effects of UV radiation on DNA and its components. J. Photochem. Photobiol. B: Biol. 2001, 63, 88-102.

31. Foote, C. S., Definition of Type I and Type II. Photochem. Photobiol. 1991, 54, 659.

32. Cadet, J.; Douki, J.; Ravanat, J.; Mascio, P., Sensitized formation of oxidatively generated damage to cellular DNA by UVA radiation. Photochem. Photobiol. 2009, 8, 903-911.

33. Gniadecki, R. T.; Thorn, T.; Vicanova, J.; Petersen, A. B.; Wulf, H. C., Role of mitochondria in ultraviolet-induced oxidative stress. J. Cell. Biochem. 2000, 80, 216-222. 
34. Pourzand, C.; Watkin, R. D.; Brown, J. E.; Tyrell, R. M., Ultraviolet A radiation induces immediate release of iron in human primary skin fibroblasts: the role of ferritin. Proc. Natl. Acad. Sci. 1999, 96, 6751-6756.

35. Cadet, J.; Delatour, T.; Douki, T.; Gasparutto, D.; Pouget, J.; Ravanat, J.; Sauvagio, S., Hydroxyl radicals and DNA base damage. Mutation Research 1999, 424, 921.

36. Pogozelski, W. K.; Tullius, T. D., Oxidative strand scission of nucleic acids: routes initiated by hydrogen abstraction from the sugar moiety. Chem. Rev. 1998, 98, 1089-1107.

37. Cadet, J.; Ravanat, J. L.; Martinez, G. R.; Medeiros, M. H.; Mascio, P., Singlet oxygen oxidation of isolated and cellular DNA: product formation and mechanistic insights. Photochem. Photobiol. 2006, 82, 1219-1225.

38. Bosca, F.; Lhiaubet, V.; Cuquerella, M. C.; Castell, J. V.; Miranda, M. A., The triplet energy of thymine in DNA. J. Am. Chem. Soc. 2006, 128, 6318-6319.

39. Cuquerella, M. C.; Lhiaubet-Vallet, V.; Cadet, J.; Miranda, M. A., Benzophenone photosensitized DNA damage. Acc. Chem. Res. 2012, 45, 1558-1570.

40. Lhiaubet-Vallet, V.; Cuquerella, M. C.; Castell, J. V.; Bosca, F.; Miranda, M. A., Triplet excited fluoroquinolones as mediators for thymine cyclobutane dimer formation in DNA. J. Phys. Chem. B 2007, 111, 7409-7414.

41. Vendrell-Criado, V.; Rodríguez-Muñiz, G. M.; Cuquerella, M. C.; LhiaubetVallet, V.; Miranda, M. A., Photosensitization of DNA by 5-methyl-2-pyrimidone deoxyribonucleoside: (6-4) photoproduct as a possible Trojan horse. Angew. Chem. Int. Ed. 2013, 52, 1-5.

42. Aparici-Espert, I.; Garcia-Lainez, G.; Andreu, I.; Miranda, M. A.; LhiaubetVallet, V., Oxidatively generated lesions as internal photosensitizer for pyrimidine dimerization in DNA. ACS Chem. Biol. 2018, 13, 542-547. 
43. Le May, N. E., J. M.; Coin, F., True lies: the double life of the nucleotide excision repair factors in transcription and DNA repair. J. Nucleic acids 2010, 2010, 1 10.

44. Liu, Y. P., R.; Beard, W. A.; Kedar, P. S.; Hou, E. W.; Shock, D. D.; Wilson, S. H., Coordination of steps in single-nucleotide base excision repair mediated by apurinic/apyrimidinic endonuclease 1 and DNA polymerase beta. J. Biol. Chem. 2007, $282,13532-13541$.

45. Sancar, A., Structure and function of DNA photolyase and cryptochrome blue-light photoreceptors. Chem. Rev. 2003, 103, 2203-2207.

46. Fousteri, M.; Mullenders, L., Transcription-coupled nucleotide excision repair in mammalian cells: molecular mechanisms and biological effects. Cell Research 2008, $18,73-85$.

47. Gillet, L. C.; Schärer, O. D., Molecular mechanisms of mammalian global genome nucleotide excision repair. Chem. Rev. 2006, 106, 253-276.

48. Perdiz, D.; Gróf, P.; Mezzina, M.; Nikaido, O.; Moustacchi, E.; Sage, E., Distribution and repair of bipyrimidine photoproducts in solar UV-irradiated mammalian cells. J. Biol. Chem. 2000, 275, 26732-26742.

49. Courdavault, S.; Baudouin, C.; Charveron, M.; Canguilhem, B.; Favier, A.; Cadet, J.; Douki, T., Repair of the three main types of bipyrimidine DNA photoproducts in human keratocytes exposed to UVB and UVA radiations. DNA repair 2005, 4, 836-844.

50. Mouret, S.; Charveron, M.; Favier, A.; Cadet, J.; Douki, T., Differential repair of UVB-induced cyclobutane pyrimidine dimers in cultured human skin cells and whole human skin. DNA repair 2008, 7, 704-712.

51. Hedge, M. L.; Hazra, T. K.; Mitra, S., Early steps in the DNA base excision/single-strand interruption repair pathway in mammalian cells. Cell. Res. 2008, $18,27-47$. 
52. Surjana, D.; Halliday, G. M.; Damian, D. L., Nicotinamide enhances repair of ultraviolet radiation-induced DNA damage in human keratinocytes and ex vivo skin. Carcinogenesis 2013, 34, 1144-1149.

53. Sancar, A., Structure and function of photolyase and in vivo enzymology: 50th anniversary. J. Biol. Chem. 2008, 283, 32153-32157.

54. Sancar, A., No end of history for photolyases. Science 1996, 272, 48-49.

55. Chaves, I.; Pokomy, R.; Byrdin, M.; Hoang, N.; Ritz, T.; Brettel, K.; Essen, L. O.; van der Horst, G. T.; Batschauer, A.; Ahmad, M., The cryptochromes: blue light photoreceptors in plants and animals. Annu. Rev. Plant. Biol. 2011, 62, 335-364.

56. Kim, S. T. S., A.; Essenmacher, C.; Badcock, G. T., Time-resolved EPR studies with DNA photolyase: Excited-state $\mathrm{FADH}_{0}$ abstracts an electron form Trp306 to generate $\mathrm{FADH}^{-}$, the catalytically active form of the cofactor. Proc. Natl. Acad. Sci. 1993, 90, 8023-8027.

57. Aubert, C.; Vos, M. H.; Mathis, P.; Eker, A. P.; Brettel, K., Intraprotein radical transfer during photoactivation of DNA photolyase. Nature 2000, 405, 586590.

58. Kiontke, S. G., P.; Haselsberger, R.; Batschauer, A.; Essen, L. O., Structural and evolutionary aspects of antenna chromophore usage by class II photolyases. J. Biol. Chem. 2014, 289, 19659-19669.

59. Mees, A.; Klar, T.; Gnau, P.; Hennecke, A.; Eker, A. P.; Carell, T.; Essen, O., Crystal structure of a photolyase bound to a CPD-like DNA lesion after in situ repair. Science 2004, 306, 1789-1793.

60. MacFarlane, A. W.; Stanley, R., Cis-syn thymidine dimer repair by DNA photolyase in real time. Biochemistry 2003, 42, 8558-8568.

61. Kao, Y. T.; Saxena, C.; Wang, L.; Sancar, A.; Zhong, D., Direct observation of thymine dimer repair in DNA by photolyase. Proc. Natl. Acad. Sci. 2005, 102, 16128 16132. 
62. Thiagarajan, V. B., M.; Eker, A. P.; Muller, P.; Brettel, K., Kinetics of cyclobutane thymine dimer splitting by DNA photolyase directly monitored in the UV. Proc. Natl. Acad. Sci. 2011, 108, 9402-9407.

63. Christine, K. S.; Macfarlane, A. W.; Yang, K.; Stanley, R. J., Cyclobutypyrimidine dimer base flipping by DNA photolyase. J. Biol. Chem. 2002, 277, 38339-38344.

64. Liu, Z.; Tan, C.; Guo, X.; Kao, Y. T.; Li, J.; Wang, L.; Sancar, A.; Zhong, D., Dynamics and mechanism of cyclobutane pyrimidine dimer repair by DNA photolyase. Proc. Natl. Acad. Sci. 2011, 108, 14831-14836.

65. Espagne, A.; Byrdin, M.; Eker, A. P. M.; Brettel, K., Very fast product release and catalytic turnover of DNA photolyase. ChemBioChem 2009, 10, 1777-1780.

66. Brettel, K. B., M., DNA photolyase: Is the nonproductive back electron transfer really much lower than forward transfer? Proc. Natl. Acad. Sci. 2012, 109, E1462.

67. Zhong, D.; Sancar, A.; Stuchebrukhov, A., Reply to Brettel and Byrdin: On the efficiency of DNA repair by photolyase. Proc. Natl. Acad. Sci. 2012, 109, E1463.

68. Heelis, P. F.; Shubin, L., Photoenzymic repair of the DNA 6-4 photoproduct - A density functional theory and semiempirical study. J. Am. Chem. Soc. 1997, 119, 2936-2937.

69. Hitomi, K. N., H.; Kim, S. T.; Mizukoshi, T.; Ishikawa, T.; Iwai, S.; Todo, T., Role of two histidines in the (6-4) photolyase reaction. J. Biol. Chem. 2001, 276, 1010310109.

70. Maul, M. J. B., T. R.; Glas, A. F.; Cryle, M. J.; Domratcheva, T.; Schneider, S.; Schlichting, I.; Carell, T., Crystal structure and mechanism of a DNA (6-4) photolyase. Angew. Chem. Int. Ed. 2008, 47, 10076-10080.

71. Glas, A. F.; Schneider, S.; Maul, M. J.; Hennecke, U.; Carell, T., Crystal structure of the $\mathrm{T}(6-4) \mathrm{C}$ lesion in complex with a (6-4) DNA photolyase and repair of UV-induced (6-4) and Dewar photolesions. Chemistry 2009, 15, 10387-10396. 
72. Yamamoto, J.; Plaza, P.; Brettel, K., Repair of (6-4) lesions in DNA by (6-4) photolyase: 20 years of quest for the photoreaction mechanism. Photochem. Photobiol. 2017, 93, 51-66.

73. Li, J.; Liu, Z.; Tan, C.; Guo, X.; Wang, L.; Sancar, A.; Zhong, D., Dynamics and mechanism of repair of ultraviolet-induced (6-4) photoproduct by photolyase. Nature 2010, 466, 887-890.

74. Sadeghian, K.; Bocola, M.; Merz, T.; Schüz, M., Theoretical study on the repair mechanism of the (6-4) photolesion by the (6-4) photolyase. J. Am. Chem. Soc. 2010, 132, 16285-16295.

75. Yamamoto, J.; Martin, R.; Iwai, S.; Plaza, P.; Brettel, K., Repair of the (6-4) photoproduct by DNA photolyase requires two photons. Angew. Chem. Int. Ed. 2013, 52, 7432-7436.

76. Wang, Y.; Gaspar, P. P.; Taylor, J. E., Quantum chemical study of the electron transfer-catalyzed splitting of oxetane and azetidine intermediates proposed in the photoenzymatic repair of (6-4) photoproduct of DNA. J. Am. Chem. Soc. 2000, 122, 5510-5519.

77. Rahn, R. O.; Hosszu, J. L., Photochemical studies of thymine in ice. Photochem. Photobiol. 1969, 131-137.

78. Prakash, G.; Falvey, D. E., Model studies of the (6-4) photoproduct DNA photolyase: synthesis and photosensitized splitting of a thymine-5,6-oxetane. J. Am. Chem. Soc. 1995, 117, 11375-11376.

79. Joseph, A.; Falvey, D. E., Photoinduced electron transfer cleavage of oxetane adducts of uracil and cytosine. Photochem. Photobiol. 2002, 1, 632-635.

80. Joseph, A.; Prakash, G.; Falvey, D. E., Model studies of the (6-4) photoproduct photolyase enzyme: laser flash photolysis experiments confirm radical ion intermediates in the sensitized repair of thymine oxetane adducts. J. Am. Chem. Soc. 2000, 122, 11219-11225. 
81. Cichon, M. K.; Arnorld, S.; Carell, T., A (6-4) photolyase model: repair of DNA (6-4) lesions requires a reduced and deprotonated flavin. Angew. Chem. Int. Ed. 2002, 41, 767-770.

82. Clivio, P.; Fourrey, J.; Gasche, J., DNA photodamage mechanistic studies: characterization of a thietane intermediate in a model reaction relevant to "6-4" lesions. J. Am. Chem. Soc. 1991, 113, 5481-5483.

83. Fourrey, J. L.; Jouin, P.; Moron, J., Thiocarbonyl photochemistry. III. Thiethanes obtention from 4-thiouracil derivatives. Tetrabedron Lett. 1974, 35, 30053006.

84. Zhao, X.; Liu, J.; Hsu, D. S.; Zhao, S.; Taylor, J. S.; Sancar, A., Reaction mechanism of (6-4) photolyase. J. Biol. Chem. 1997, 272, 32580-32590.

85. Friedel, M. G.; Cichon, M. K.; Carell, T., Model compounds for (6-4) photolyases: a comparative flavin induced cleavage study of oxetanes and thietanes. Org. Biomol. Chem. 2005, 3, 1937-1941. 

Chapter 2:

General Objectives 

As mentioned in the introduction, this doctoral Thesis is centered on the photorepair mechanism of UV induced DNA damage. Particularly, in the cycloreversion step that is the result of a photochemically induced electron injection from the catalytic flavin-adenosine cofactor $\mathrm{FADH}^{-}$to the lesion or to the four-membered ring intermediates proposed as a key step of CPD or (6-4) photolyase mechanism, respectively. This mechanism has been resolved for the cyclobutane pyrimidine dimer, but is still under discussion for the (6-4) photoproduct. Indeed, the study of this process is not straightforward due not only to the ultrashort (some picoseconds) lifetime of the excited flavin but also to the unstable character of the oxetane or azetidine intermediates. In this context, strategies based on the development of models to mimic the four-membered ring intermediate have to be developed to investigate the electron transfer step responsible for the photocycloreversion. Therefore, the specific objectives to be addressed are:

1) To synthesize an azetidine model related to the intermediate of 6-4PP in TC sequences. To study its photoreduction triggered by photosensitizers mimicking the flavin cofactor of the photolyase through spectroscopic, electrochemical and analytical experiments as well as computational chemistry.

2) To study the photooxidation of the azetidine model with appropiate photosensitizers and compare the obtained results with those of the reductive process.

3) To design, synthesize and perform the photoreduction of oxetane and cyclobutane thymine dimer models, as four-membered ring intermediates of 6-4PP and CPD lesions, containing 8-oxoguanosine and guanosine as intrinsic photoreductants.

4) To develop the methodology to incorporate the azetidine into DNA and to investigate its photocycloreversion using 8-oxo-2'-deoxyguanosine as intrinsic photosensitizer. To extend the study using the real photorepair machinery: the CPD and (64) photolyase enzymes. 

Chapter 3:

\section{Photoreduction of an Azetidine Model of the (6-4) Photoproduct}





\subsection{Introduction}

In spite of continuous exposure of the genetic material to radiation and genotoxic chemicals, DNA repair toolbox allows correcting lesions and safeguarding the integrity of the genome. The importance of this machinery has been highlighted by the Nobel Prize in Chemistry 2015 awarded jointly to Lindahl, Modrich and Sancar for their research into the mechanisms that cells use to repair DNA. ${ }^{1}$ Among others, it has been emphasized the importance of DNA photoreactivation catalyzed by photolyases enzymes. As mentioned previously, they repair specifically bipyrimidine lesions, ie. CPDs and 6-4PPs, through a photochemically induced electron transfer from the catalytic flavin-adenosine cofactor $\mathrm{FADH}^{-}$to the lesion or to an oxetane/azetidine intermediate. ${ }^{2-4}$ The CPD photorepair mechanism has been solved completely; however, there is still a lively discussion on the photorepair of (6-4) DNA lesions. Until now, three mechanisms have been proposed, two of them involving formation of an oxetane/azetidine intermediate triggered or not by light, followed by an electron-induced cycloreversion of this four-membered ring intermediate (Scheme 3.1, pathway $(\mathrm{A})$ and $\left.\left(\mathrm{A}^{\prime}\right)\right)$. The remaining mechanism lacks the oxetane/azetidine intermediate and consists on a direct photoreduction of the 6-4PP by the singlet excited state of the reduced flavin cofactor $\mathrm{FADH}^{-}$(Scheme 3.1, pathway(B)).

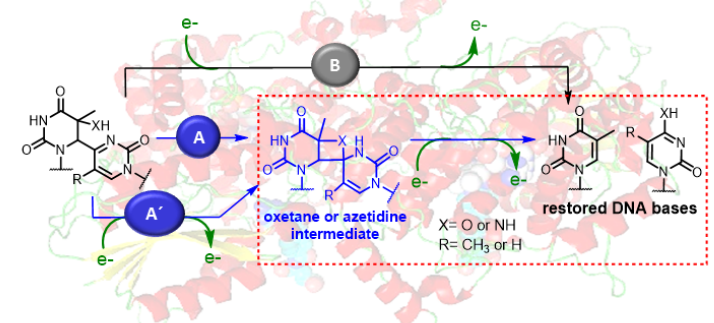

Scheme 3.1. Proposed mechanisms for the repair of the 6-4PPs by (6-4) photolyase. 
Chapter 3: Photoreduction of an Azetidine Model of the (6-4) Photoproduct

Investigation of the photorepair mechanism of 6-4PPs involves an additional diffilculty compared to the CPD lesion that is the instability of the four-membered ring oxetane/azetidine intermediates. This prevents not only their isolation and characterization but also their use as substrates to investigate the electron-induced cycloreversion. Therefore, this step has been largely studied using oxetane models (see Chapter 1$) ;{ }^{5}$ however, azetidine models have not been reported until now. The latter are particularly relevant to the understanding of (6-4) photolyase repair mechanism at TC sequences, the most frequent 6-4PP lesion.

A main difficulty to study azetidines resides in the synthesis of the model. As aforementioned, four-membered ring can be achieved through [2+2] photocycloadditions of alkenes to $\mathrm{C}=\mathrm{C}, \mathrm{C}=\mathrm{O}$ or $\mathrm{C}=\mathrm{N} \cdot{ }^{6}$ In this context, oxetane models can be easily obtained from Paternò-Büchi reaction between the benzophenone carbonyl group and the double bond of the thymine ${ }^{7}$, whereas the photochemical formation of the azetidine ring is only obtained from a "blocked" imine and an alkene. This means that the imino group must form part of a five- or six-membered ring to give rise to photochemical reactions such as photocycloaddition. The reason is ascribed to the fact that in their excited state the imino groups have a very fast deactivation due to the "twist" movement that takes place at the carbon-nitrogen double bond through which the syn and anti isomers of imines are interconverted when it is not confined in a ring system.

However, this condition alone is not sufficient for imine photoreactivity and a conjugated electron-withdrawing group like a carbonyl group or a heteroatom attached to the C-N double bond is required for cycloaddition to occur.6,8 Theoretical calculations with isoxazolines showed that electron-withdrawing groups destabilize the fast IC pathway from $S_{1}$, increasing the energy barrier. This way deactivation to ground state is slow enough to allow the system to spend time in $S_{1}$ where cycloaddition to alkenes is possible. ${ }^{9}$ Therefore, it has been reported that the organic compounds suitable for the formation of azetidines by photocycloaddition are heterocy- 
cles such as oxadiazoles $(\mathrm{OXA})^{10}$, isoindolenones $(\mathrm{INDO})^{11}$, isoxazolines $(\mathrm{AZO})^{8}$ and 6-azauracils (azaU) ${ }^{12}$ (Figure 3.1).

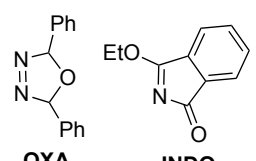

OXA INDO

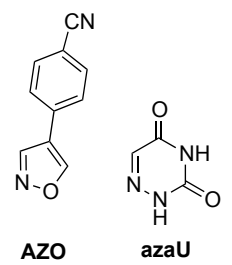

Figure 3.1. Imine compounds suitable for [2+2] photocycloadditions with alkenes.

With this background, this chapter proposes to study the photoreductive cycloreversion of an azetidine model in relation to the repair of 6-4PPs in DNA. In this context, we have chosen azaU that contains an imino group in a 6-membered ring, as a starting imine compound for the synthesis. In addition, this compound is of great interest since (i) it can be considered as a modified pyrimidine base of DNA, (ii) it has been used as a growth inhibitor in microorganisms and (iii) it shows better photochemical reactivity than canonical nucleobases, i.e. it presents an efficient ISC to $\mathrm{T}_{1}$ $\left(\pi \pi^{*}\right)$ being this process the dominant pathway in the relaxation of the $S_{1} \cdot{ }^{13}$

Interestingly, the photoreductive approach has only been applied to the ring splitting of azetidin-2-ones using triethylamine or DABCO as electron donors (Scheme 3.2). However, these compounds cannot be formally considered as azetidines as the nitrogen atom belongs to a strained $\beta$-lactam. ${ }^{14-15}$ 


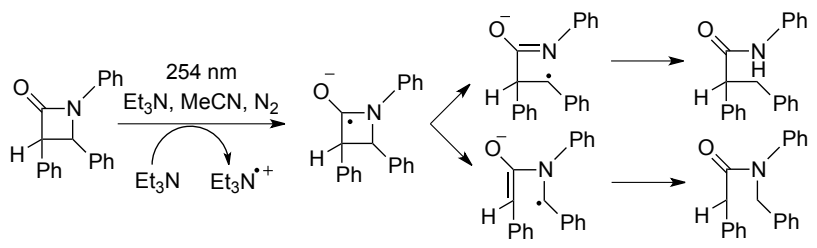

Scheme 3.2. Mechanistic pathways for ring splitting of an azetidin-2-one derivative in the presence of triethylamine.

Thus, in this chapter a photoreductive approach has been applied to the ring splitting of a stable azabipyrimidinic azetidine $\mathbf{T}_{\mathrm{m}}<>\mathbf{A Z} \mathbf{T}_{\mathrm{m}}$ (Figure 3.2), where the nitrogen atom belongs to the modified base azaU, as a close model for the intermediate $\mathbf{T}(\mathbf{a z t}) \mathbf{C}$ proposed for the repair of 6-4PPs at TC sequences. Parallel studies of photoinduced reductive cycloreversion on related cyclobutane and azetidine derivatives $\left(\mathbf{T}_{\mathrm{m}}<>\mathbf{T}_{\mathrm{m}}\right.$ and $\mathbf{D C H}<>\mathbf{A Z} \mathbf{T}_{\mathrm{m}}$, Figure 3.2) have been included to ascertain how the presence of the nitrogen atom in the four-membered ring affects in the ring splitting.
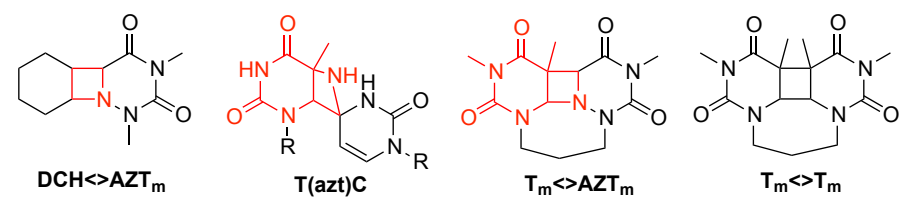

Figure 3.2. Structures of the azetidine model $\mathbf{T}_{\mathrm{m}}<>\mathbf{A Z} \mathbf{T}_{\mathrm{m}}$ studied in this chapter related to the intermediate $\mathbf{T}(\mathbf{a z t}) \mathbf{C}$ involved in the photorepair mechanism of 64PPs at TC sequences and the cyclobutane and azetidine derivatives $\mathbf{T}_{\mathrm{m}}<>\mathbf{T}_{\mathrm{m}}$ and $\mathrm{DCH}<>\mathrm{AZT}_{\mathrm{m}}$. 


\subsection{Results and discussion}

\subsubsection{Synthesis}

We decided to synthesize the dinucleotide azetidine model $\mathbf{T}_{\mathrm{m}}<>\mathbf{A Z} \mathbf{T}_{\mathrm{m}}$ in which 6-azauracil is connected with thymine through a trimethylene bridge. This previously reported compound ${ }^{16-18}$ is a simplified model without phosphate backbone or sugar ring conformations, which eases the study of ring cycloreversion. However, $\mathbf{T}_{\mathrm{m}}<>\mathbf{A} \mathbf{Z} \mathbf{T}_{\mathrm{m}}$ was obtained by alternative synthesis from thymine as shown in the Scheme 3.3. Due to the low solubility of 6-azauracil and thymine in most common organic solvents, soluble 1,3-dimethyl derivatives were employed. The synthesis of $\mathbf{T}_{\mathrm{m}}-\mathbf{a z a} \mathbf{U}_{\mathrm{m}}$ was achieved by reaction of 1-bromo-3-chloropropane with N3methylthymine 1 and subsequent reaction of the resulting 1-(3-chloropropyl)-3methylthymine 2 with the N3-methyl derivative of 6-azauracil. Then, $\mathbf{T}_{\mathrm{m}}<>\mathbf{A Z} \mathbf{T}_{\mathrm{m}}$ was obtained as the main photoproduct by irradiation of $\mathbf{T}_{\mathrm{m}}-\mathbf{a z a} \mathbf{U}_{\mathrm{m}}$ at $\boldsymbol{\lambda}>290 \mathrm{~nm}$ in the presence of acetone as photosensitizer, which was used to avoid photocycloreversion of the product under direct irradiation.

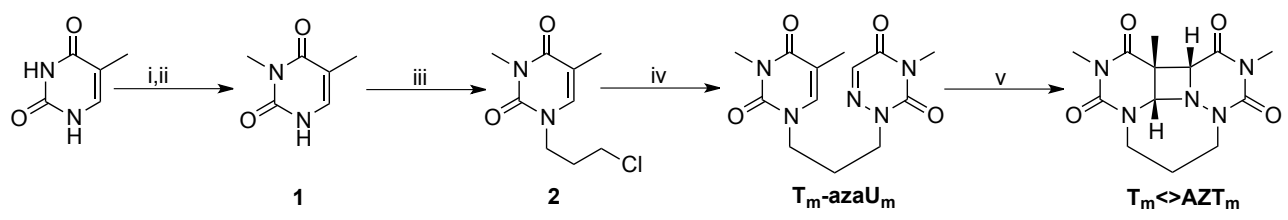

Scheme 3.3. Synthetic strategy to prepare $\mathbf{T}_{\mathrm{m}}<>\mathbf{A Z} \mathbf{T}_{\mathrm{m}}$. Reagents and conditions: (i) $\mathrm{Ac}_{2} \mathrm{O}, 140{ }^{\circ} \mathrm{C}, 5 \mathrm{~h}$; (ii) $\mathrm{MeI}, \mathrm{NaH}, \mathrm{DMF}, \mathrm{rt}, 18 \mathrm{~h}$; (iii) 1-bromo-3chloropropane, $\mathrm{NaH}, \mathrm{DMF}$, rt, 18h; (iv) N3-methyl-6-azauracil, $\mathrm{NaH}, \mathrm{MeCN}, 80^{\circ} \mathrm{C}$, 18h; (v) hv, $\lambda>290 \mathrm{~nm}$, acetone, $1 \mathrm{~h}$. 
Chapter 3: Photoreduction of an Azetidine Model of the (6-4) Photoproduct

Therefore, the intramolecular [2+2] cycloaddition of $\mathbf{T}_{\mathbf{m}}-\mathbf{a z a} \mathbf{U}_{\mathrm{m}}$ occurs through a photosensitized reaction (Scheme 3.4) where acetone after absorbing light, reaches its singlet excited state $\left({ }^{1} \mathrm{AC}^{*}\right)$ and quantitatively crosses to its triplet state $\left({ }^{3} \mathrm{AC}^{*}\right)$. Then, TTET occurs to populate the triplet excited state of azaU ( $\left.{ }^{3} \mathrm{azaU}^{*}\right)$, which reacts with the linked thymine to form the azetidine ring. Here, acetone is a suitable sensitizer, as its triplet energy $\left(337 \mathrm{~kJ} \mathrm{~mol}^{-1}\right)^{19}$ is higher than that of azaU $\left(324 \mathrm{~kJ} \mathrm{~mol}^{-1}\right)^{20}$, which was estimated by phosphorescence emission measurements. In addition, high yield for the photocycloaddition was obtained since (i) the quantum yield of ${ }^{3} \mathrm{azaU}^{*}$ formation was estimated to be unity by acetone triplet sensitization, which is five times larger than that of uracil13 and, (ii) the trimethylene bridge favors the rate of internal dimerization. ${ }^{21-22}$

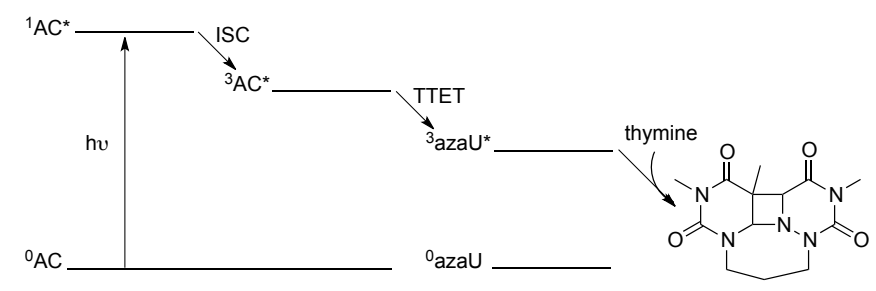

Scheme 3.4. Intramolecular $[2+2]$ cycloaddition between aza-U and thymine through photosensitized pathway.

Once purified, the product lacks the $270 \mathrm{~nm}$ absorption (Figure 3.3, A) in agreement with the saturation of the C5-C6 and C5-N6 double bonds. Accordingly, photoreversion is observed when the product is irradiated at $\lambda=254 \mathrm{~nm}$ (Figure 3.3, B) through appearance of the UV absorption at $270 \mathrm{~nm}$ of pyrimidine analogs, supporting the formation of an aza-cyclobutane ring. 

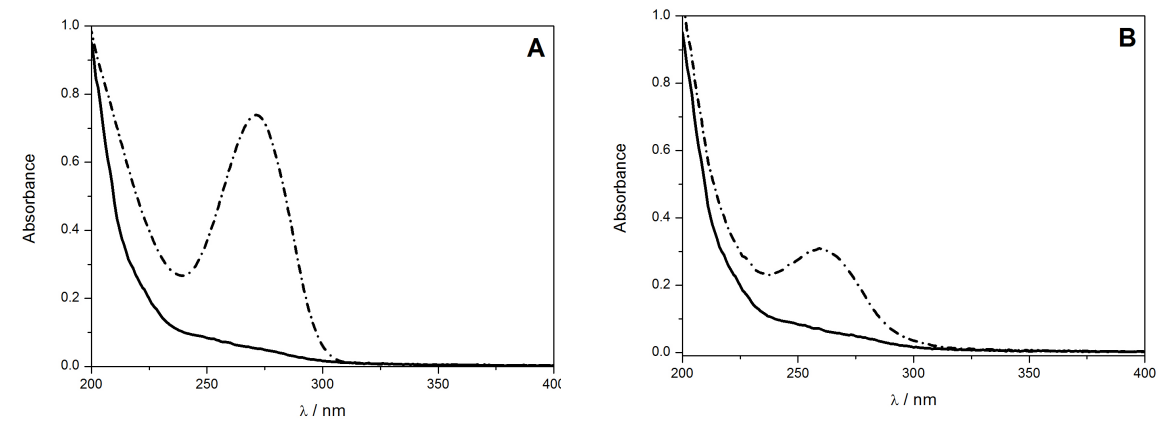

Figure 3.3. (A) UV absorption spectra of $\mathbf{T}_{\mathrm{m}}<>\mathbf{A Z} \mathbf{T}_{\mathrm{m}}\left(1 \times 10^{-5} \mathrm{M}\right.$, solid line) and $\mathbf{T}_{\mathrm{m}}-\mathbf{a z a U}_{\mathrm{m}}\left(5 \times 10^{-5} \mathrm{M}\right.$, short dash dot line) in acetonitrile. (B) UV absorption spectra of an acetonitrile solution of $\mathbf{T}_{\mathbf{m}}<>\mathbf{A Z} \mathbf{T}_{\mathbf{m}}$ before (solid line) and after (short dash dot line) irradiation for $30 \mathrm{~min}$ at $254 \mathrm{~nm}$.

The intramolecular photodimerization of $\mathbf{T}_{\mathrm{m}}-\mathbf{a z a} \mathbf{U}_{\mathrm{m}}$ leds to only one photoproduct as no traces of other photoisomers have been observed. The cis-syn configuration of $\mathbf{T}_{\mathrm{m}}<>\mathbf{A} \mathbf{Z} \mathbf{T}_{\mathrm{m}}$ was determined by NOE experiments (Figure 3.4) through the interaction of azetidine ring protons $\mathrm{H}_{\mathrm{a}}$ and $\mathrm{H}_{\mathrm{b}}$ with the C5-methyl group of the dihydrothymine moiety. The cis-anti and trans-anti isomers cannot be formed because of length of trimethylene chain. In addition, the structure of photodimer is also supported by the base-base stacking effect that should favor the formation of $c i s-s y n$ isomer. ${ }^{23}$ Thermal stability of $\mathbf{T}_{\mathrm{m}}<>\mathbf{A Z} \mathbf{T}_{\mathrm{m}}$ in acetonitrile was also evaluated by UV/VIS measurements at 298K, and no spectral changes were detected after $48 \mathrm{~h}$. 
Chapter 3: Photoreduction of an Azetidine Model of the (6-4) Photoproduct

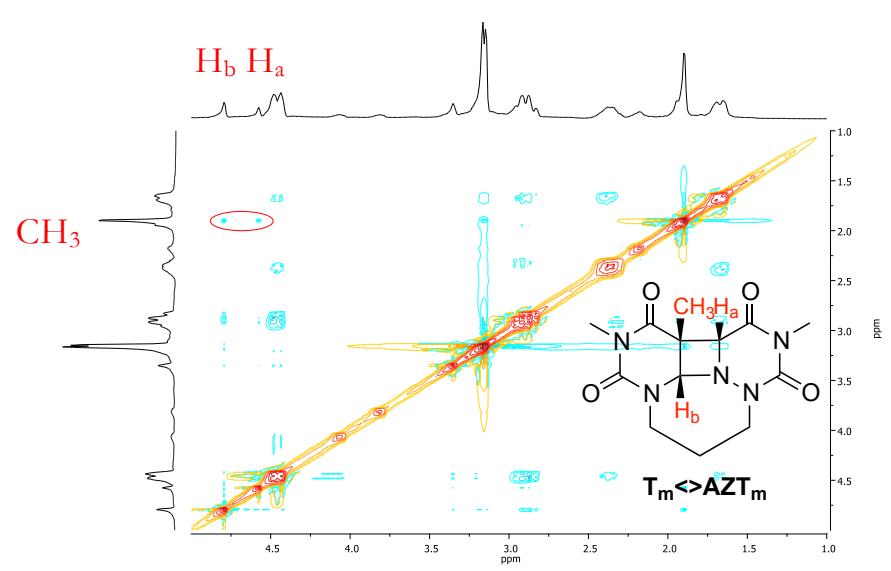

Figure 3.4 NOESY spectrum of $\mathbf{T}_{\mathrm{m}}<>\mathrm{AZT}_{\mathrm{m}}$.

The related cyclobutane derivative $\mathbf{T}_{\mathrm{m}}<>\mathbf{T}_{\mathrm{m}}$ was prepared by a three-step synthesis from the commercial O,O'-bis(trimethylsilyl)-thymine, as shown in Scheme 3.5. The synthesis of $\mathbf{T}_{\mathrm{m}}-\mathbf{T}_{\mathrm{m}}$ was achieved by reaction of 1,3-dibromopropane with O,O'bis(trimethylsilyl)-thymine followed of the $\mathrm{N} 3$ methylation. Then, $c i s-\operatorname{syn} \mathbf{T}_{\mathrm{m}}<>\mathbf{T}_{\mathrm{m}}$ was obtained by an intramolecular [2+2] cycloaddition of $\mathbf{T}_{\mathbf{m}}-\mathbf{T}_{\mathrm{m}}$ in the presence of acetone as mentioned before.

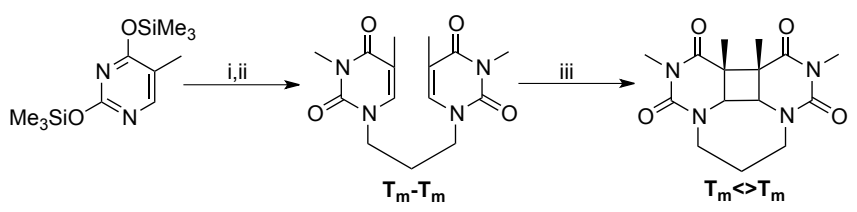

Scheme 3.5. Synthetic strategy to prepare $\mathbf{T}_{\mathrm{m}}<>\mathbf{T}_{\mathrm{m}}$. Reagents and conditions. (i) 1,3-dibromopropane, DMF, $170{ }^{\circ} \mathrm{C}, 18 \mathrm{~h}$; (ii) MeI, NaH, DMF, rt, 18h; (iii) hv, $\lambda>$ $290 \mathrm{~nm}$, acetone, $2 \mathrm{~h}$. 
Another azetidine model $\left(\mathbf{D C H}<>\mathbf{A} \mathbf{Z} \mathbf{T}_{\mathrm{m}}\right)$ was synthetized where the thymine moiety of $\mathbf{T}_{\mathrm{m}}<>\mathbf{A Z} \mathbf{T}_{\mathrm{m}}$ was replaced with a cyclohexane ring. In this case, two azetidine isomers cis and trans $\mathbf{D C H}<>\mathbf{A Z T}_{\mathbf{m}}$ were obtained from 6-aza-1,3dimethyluracil 4 through an acetone photosensitized reaction in the presence of cyclohexene as previously reported ${ }^{12}$ (Scheme 3.6.).

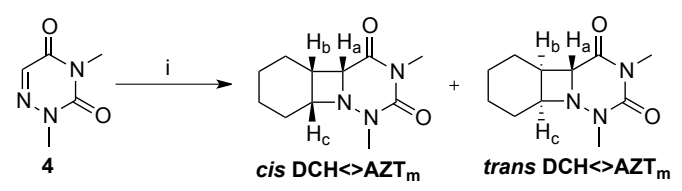

Scheme 3.6. Synthesis of cis and trans $\mathbf{D C H}<>\mathbf{A Z} \mathbf{T}_{\mathrm{m}}$. Reagents and conditions: (i) $\mathrm{h} v, \boldsymbol{\lambda}>290 \mathrm{~nm}$, acetone, $6 \mathrm{~h}$

The two isomers were easily separated by flash chromatography. Their stereochemistry was determined from NMR analysis. A tentative assignment was proposed from the ${ }^{1} \mathrm{H}$ NMR spectra because, in the case of the cis isomer, the $\mathrm{H}_{a}, \mathrm{H}_{\mathrm{b}}$ and $\mathrm{H}_{\mathrm{c}}$ protons are located on the same side of the azetidine ring. Thus, $\mathrm{H}_{\mathrm{a}}$ should be observed as a doublet as it is coupled to $\mathrm{H}_{\mathrm{b}}$. By contrast, in the trans isomer, $\mathrm{H}_{\mathrm{a}}$ does not interact with the other two protons of the ring, and thus appears as a singlet at ca. $4.0 \mathrm{ppm}$. However, this signal cannot be observed clearly as it overlaps with $\mathrm{H}_{\mathrm{c}}$. The NOESY 2D NMR spectrum of cis $\mathbf{D C H}<>\mathbf{A Z} \mathbf{T}_{\mathbf{m}}$ is in agreement with the proposed cis configuration as an interaction was observed between $\mathrm{H}_{\mathrm{a}}$ and $\mathrm{H}_{\mathrm{b}}$ protons located at 4.33 and $4.01 \mathrm{ppm}$, respectively (Figure 3.5). 


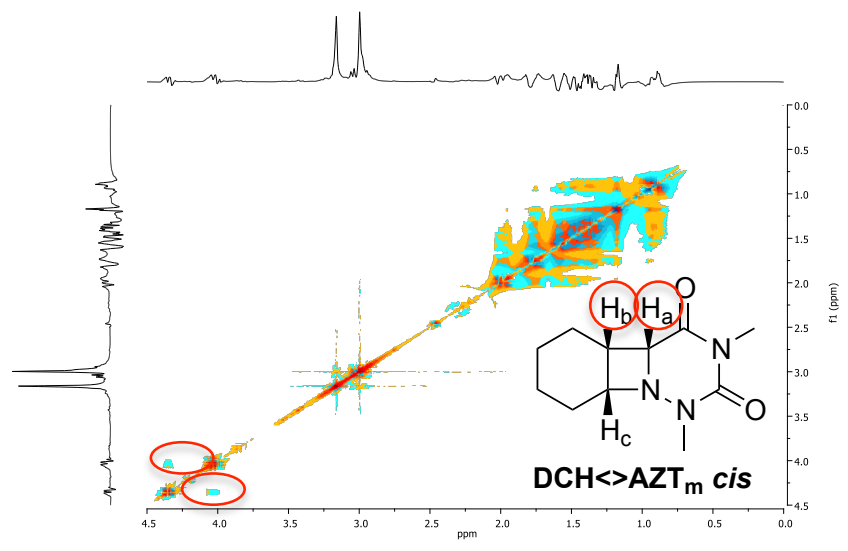

Figure 3.5. NOESY spectrum of $c$ is $\mathbf{D C H}<>\mathbf{A Z T}_{\mathrm{m}}$.

\subsubsection{Fluorescence experiments}

The trimethylene bridge used for $\mathbf{T}_{\mathbf{m}}<>\mathbf{A Z} \mathbf{T}_{\mathrm{m}}$ has been previously used in formation ${ }^{21-23}$ and repair ${ }^{24-26}$ studies of CPD models, and it appears to favor the interaction of the lesion with flavin singlet excited state that points to a relatively long lifetime of the dimer radical (cation or anion) involved which is presumably due to stabilization. ${ }^{26-27}$ It is assumed that the electron transfer process occurs from the singlet excited state of the reduced flavin $\mathrm{FADH}^{-}$to the azetidine moiety. Thus, direct mechanistic information shoud, in principle, be obtained by monitoring the changes in the intensity and/or kinetics of the cofactor emission in the presence of the azetidine by steady-state and/or time-resolved fluorescence, respectively.

However, the very short lifetime of excited $\mathrm{FADH}^{-}$(in the subnanosecond timescale) does not provide a time-window compatible with diffusion-controlled intermolecular reaction..$^{28}$ To overcome this limitation, a series of photosensitizers (PS, Figure 3.6) with singlet lifetime in the nanosecond range (see $\tau$, Table 3.1) and oxidation potential close to that of ${ }^{1} \mathrm{FADH}^{-*}\left(\mathrm{E}_{\mathrm{ox}}^{\mathrm{D} *} \text { of ca. }-2.9 \mathrm{~V} \text { vs. } \mathrm{Ag} / \mathrm{AgCl}\right)^{29}$ were selected (Table 3.1).24-25, 30 They include N,N,N',N'-tetramethyl-1,4-phenyldiamine (TMPD), 
N,N,N',N'-tetramethylbenzidine (TMB), N,N-dimethylaniline (DMA), carbazole (CAR), acenaphthene (ACE), 1-methoxynaphthalene (1-MN), 2-methoxynaphthalene (2-MN) and chrysene (CHRY).

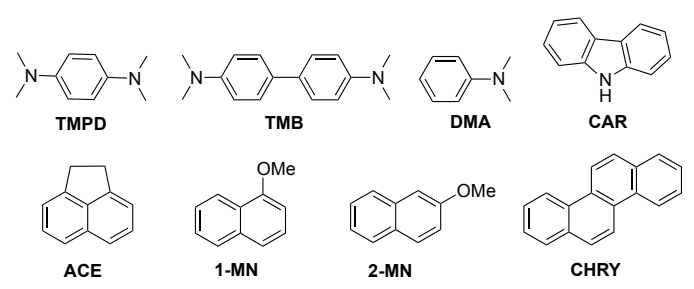

Figure 3.6. Structure of the used photosensitizers (PS).

Table 3.1. Oxidation potential $\left(\mathrm{E}_{\mathrm{ox}}^{\mathrm{D}}\right)$ and oxidation potential in the singlet excited state $\left(\mathrm{E}_{\mathrm{ox}}^{\mathrm{D}^{*}}\right)$ of the selected PS, and bimolecular rate constant $\left(\mathrm{k}_{\mathrm{q}}\right)$ for the quenching of the PS by $\mathbf{T}_{\mathrm{m}}<>\mathbf{A Z Z} \mathbf{T}_{\mathrm{m}}$ determined by time-resolved fluorescence.

\begin{tabular}{c|ccccc}
$\mathbf{P S}$ & $\begin{array}{c}\mathbf{E}_{\mathbf{o x}}^{\mathbf{D}^{*}} / \mathbf{V} \mathbf{v s .} \\
\mathbf{A g} / \mathbf{A g C l}\end{array}$ & $\begin{array}{c}\mathbf{E}_{\mathbf{o x}}^{\mathbf{D}} / \mathbf{V} \mathbf{v s .} \\
\mathbf{A g} / \mathbf{A g C l}\end{array}$ & $\begin{array}{c}\mathbf{E}^{*} / \mathbf{k c a l} \\
\mathbf{m o l}^{-1}\end{array}$ & $\boldsymbol{\tau} / \mathbf{n s}$ & $\mathbf{k}_{\mathbf{q}}[a] / \mathbf{x} 10^{9} \mathbf{M}^{-1} \mathbf{s}^{-1}$ \\
\hline TMPD & $-3.3^{24}$ & 0.2 & 79.7 & 1.5 & N.D.[b] \\
TMB & $-3.2^{24}$ & 0.4 & 83.2 & 6.0 & $8.0 \pm 0.7$ \\
DMA & $-3.0^{24}$ & 0.8 & 89.4 & 2.8 & $7.3 \pm 1.0$ \\
FADH & -2.925 & & & & \\
CAR & $-2.5^{30}$ & 1.2 & 83.1 & 7.4 & $4.0 \pm 0.2$ \\
ACE & -2.524 & 1.4 & 89.9 & 10.6 & $3.7 \pm 0.2$ \\
1-MN & $-2.5^{25}$ & 1.4 & 89.0 & 6.2 & $4.0 \pm 0.3$ \\
2-MN & -2.324 & 1.4 & 85.5 & 6.6 & $2.5 \pm 0.2$ \\
CHRY & -2.124 & 1.3 & 79.3 & 11.9 & $1.7 \pm 0.2$
\end{tabular}

[a] The experiments were performed twice and the errors correspond to average deviations. [b] Not determined because of the temporal resolution of the setup. 
In this way, the PS is an excited-state electron donor, which after absorption of a photon $\left({ }^{1} \mathrm{PS}^{*}\right)$, donates an electron to generate the radical anion in the carbonyl group of the thymine or azaU moiety of $\mathbf{T}_{\mathrm{m}}<>\mathbf{A Z} \mathbf{T}_{\mathrm{m}}$, leading finally to the repaired bases after $\mathrm{C}-\mathrm{C}$ bond cleavage as shown in Scheme 3.7. Comparison of the fluorescence experiments performed with $\mathbf{T}_{\mathrm{m}}<>\mathbf{A Z} \mathbf{T}_{\mathrm{m}}, \mathbf{T}_{\mathrm{m}}<>\mathbf{T}_{\mathrm{m}}$ and $\mathbf{D C H}<>\mathbf{A Z} \mathbf{T}_{\mathrm{m}}$ would inform on the site for the radical anion formed and on which moiety of $\mathbf{T}_{\mathrm{m}}<>\mathbf{A Z} \mathbf{T}_{\mathrm{m}}$ is involved in the electron transfer mechanism.

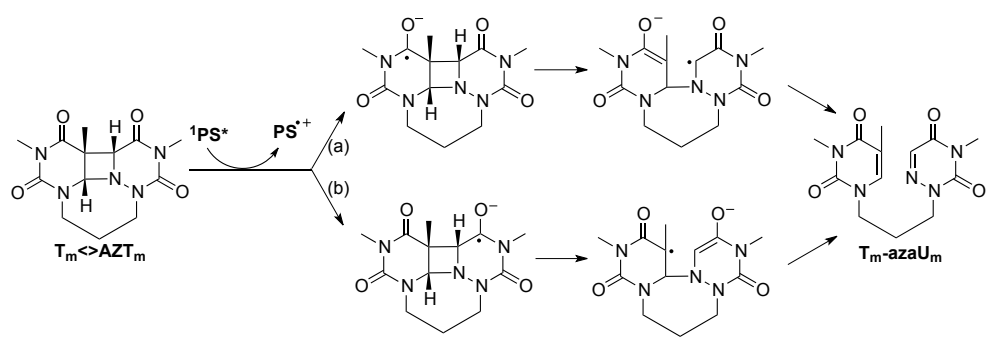

Scheme 3.7. Reductive PET mechanisms of $\mathbf{T}_{\mathrm{m}}<>\mathbf{A Z} \mathbf{T}_{\mathrm{m}}$ in the presence of PS.

In a first stage, steady-state fluorescence experiments were performed to support the above mentioned PET mechanism from the singlet excited state of PS. Thus, acetonitrile solutions of the photosensitizer were prepared with an absorbance of ca. 0.15 at the excitation wavelength $\left(\lambda_{\text {exc }}=310\right.$ or $\left.375 \mathrm{~nm}\right)$ and the fluorescence intensity was measured in the absence and in the presence of $\mathbf{T}_{\mathrm{m}}<>\mathbf{A Z Z} \mathbf{T}_{\mathrm{m}}$. Steady-state fluorescence measurements revealed a decrease of the emission intensity for all the photosensitizers, especially for TMB and DMA (Figure 3.7b and 3.7c). In these cases, the concentrated quencher absorbs partial light when the sample is excited at $310 \mathrm{~nm}$, reducing the portion of light absorbed by the photosensitizer, which thus results in a strong decrease of the emission intensity. 

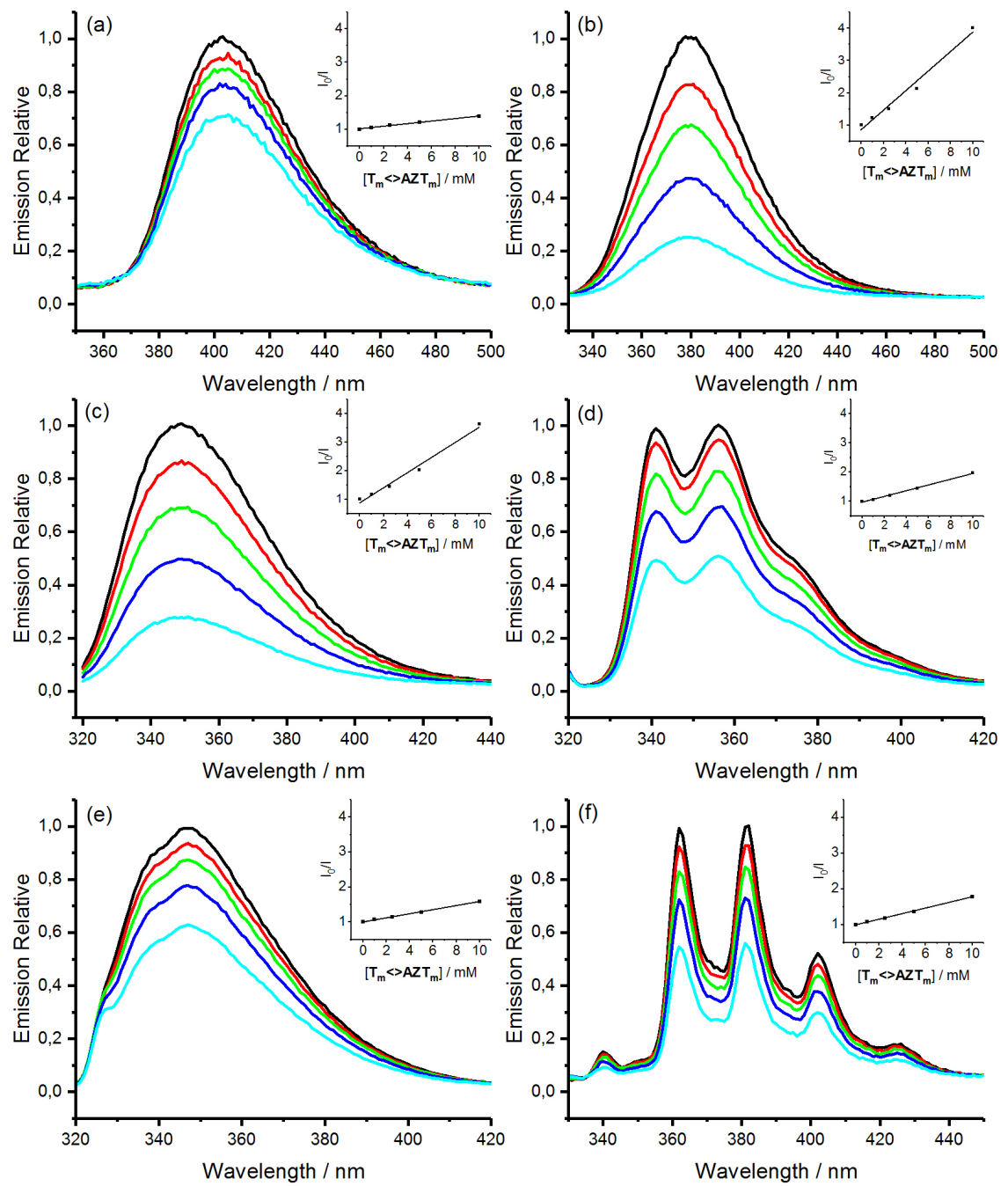

Figure 3.7. Fluorescence emission spectra for (a) TMPD $\left(\lambda_{\text {exc }}=330 \mathrm{~nm}\right)$; (b) $\operatorname{TMB}\left(\lambda_{\text {exc }}=310 \mathrm{~nm}\right) ;(\mathrm{c})$ DMA $\left(\lambda_{\text {exc }}=310 \mathrm{~nm}\right) ;(\mathrm{d}) \operatorname{CAR}\left(\lambda_{\text {exc }}=320 \mathrm{~nm}\right) ;(\mathrm{e}) 2-\mathrm{MN}$ $\left(\lambda_{\text {exc }}=328 \mathrm{~nm}\right)$; (f) CHRY $\left(\lambda_{\text {exc }}=320 \mathrm{~nm}\right)$; in the presence of increasing amounts of $\mathbf{T}_{\mathrm{m}}<>\mathbf{A Z T}_{\mathrm{m}}(0-10 \mathrm{mM})$. Inset: corresponding Stern-Volmer plot. 
To evaluate if a dynamic quenching was taking place, i.e. that the decrease of the fluorescence intensity is not due to a filter effect or to preassociation between PS and azetidine before excitation, time-resolved fluorescence experiments were performed. The singlet excited state lifetime of all photosensitizers was shortened in the presence of $\mathbf{T}_{\mathrm{m}}<>\mathbf{A Z} \mathbf{T}_{\mathrm{m}}$ (Figure 3.8). The bimolecular rate constants $\mathrm{k}_{\mathrm{q}}$ were determined from the Stern-Volmer plots representing the reciprocal of the PS lifetime as a function of $\mathbf{T}_{\mathrm{m}}<>\mathbf{A Z} \mathbf{T}_{\mathrm{m}}$ concentration (Figure 3.8, inset). According to the relationship given in equation 3.1 between $\mathrm{k}_{\mathrm{q}}$ and $\Delta \mathrm{G}_{\mathrm{ct}}$ obtained from Rehm Weller equation 3.2, the quenching process was more efficient as $\mathrm{E}_{\mathrm{ox}}^{\mathrm{D}^{*}}$ became increasingly negative (Table 3.1 and Figure 3.9 left), reaching the diffusion limit near $-3.0 \mathrm{~V}$.

By contrast, no clear correlation was obtained between $\mathrm{k}_{\mathrm{q}}$ and the PS singlet excited state energy $\mathrm{E}^{*}$ (Figure 3.9 right) ruling out a singlet-singlet energy transfer process as responsible for the deactivation of ${ }^{1} \mathrm{PS}^{*}$. Altogether, these data point to an electron transfer mechanism between ${ }^{1} \mathrm{PS}^{*}$ and $\mathbf{T}_{\mathrm{m}}<>\mathbf{A Z Z} \mathbf{T}_{\mathrm{m}}$. It is noticeable that $\mathrm{E}_{\mathrm{ox}}^{\mathrm{D} *}$ for the $\mathrm{FADH}^{-}$photolyase cofactor is of ca. $-2.9 \mathrm{~V} ; 25$ this value, when included in Figure 3.9 left, corresponds to a rate constant for the electron transfer process with $\mathbf{T}_{\mathrm{m}}<>\mathbf{A Z T}_{\mathrm{m}}$ of ca. $6 \times 10^{9} \mathrm{M}^{-1} \mathrm{~s}^{-1}$.

$$
\begin{gathered}
k_{q}=\frac{k_{\text {diff }}}{1+0.25\left\{\exp \left(\frac{\Delta G_{c t}^{f}}{R T}\right)+\exp \left(\frac{\Delta G_{c t}}{R T}\right)\right\}} \\
\text { where } \Delta G_{c t}=23.06\left(E_{\text {ox }}^{D *}-E_{r e d}^{A}\right)
\end{gathered}
$$



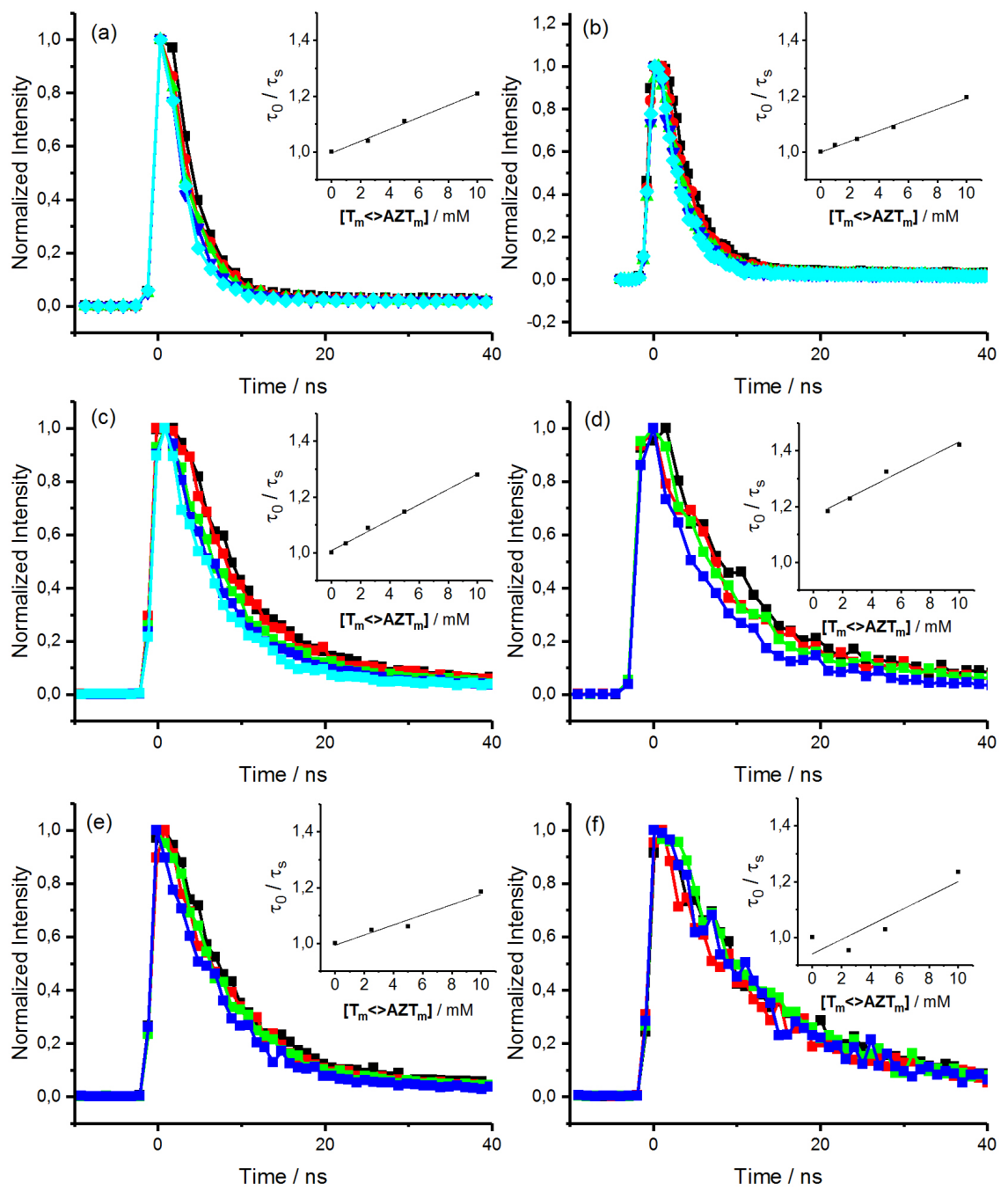

Figure 3.8. Fluorescence kinetic traces and their corresponding Stern-Volmer plot obtained for (a) TMB, (b) DMA, (c) CAR, (d) ACE, (e) 2-MN, (f) CHRY in the presence of increasing amounts of $\mathbf{T}_{\mathbf{m}}<>\mathbf{A Z} \mathbf{T}_{\mathrm{m}}$ (from 0 to $10 \mathrm{mM}$ ), upon excitation at $310 \mathrm{~nm}$. 


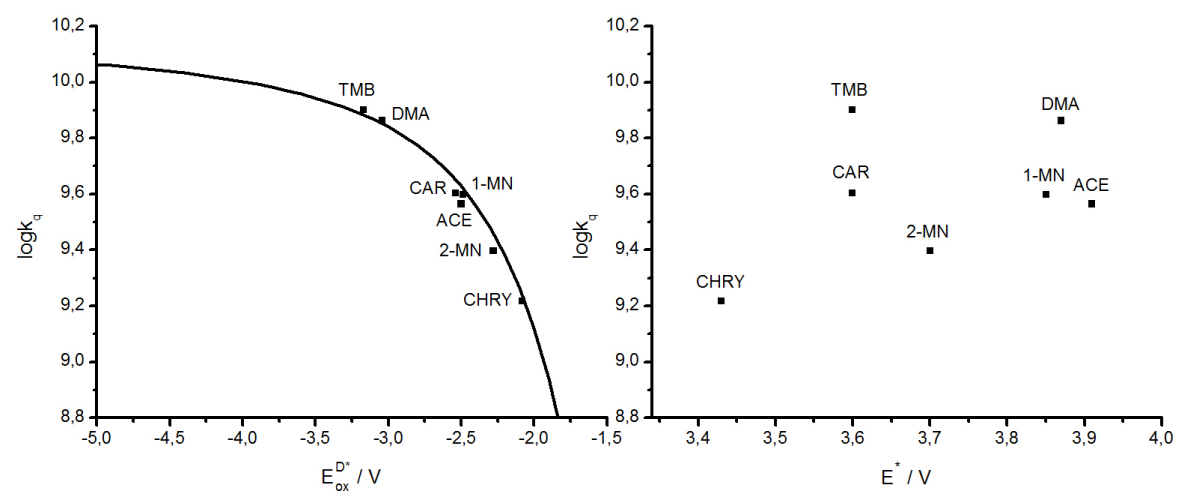

Figure 3.9. (Left) Representation of $\log k_{\mathrm{q}}$ obtained by time resolved fluorescence versus singlet excited state oxidation potential $\left(\mathrm{E}_{\mathrm{ox}}^{\mathrm{D}^{*}}\right)$ and (right) singlet excited state energy of the considered photosensitizers $\left(E^{*}\right)$.

To compare the influence of the aza group in the four-membered ring splitting, fluorescence experiments were performed for $\mathbf{T}_{\mathrm{m}}<>\mathbf{T}_{\mathrm{m}}$ (Table 3.2, Figure 3.10). In this context, 1-MN and CHRY were selected as photosensitizers. As for $\mathbf{T}_{\mathrm{m}}<>\mathbf{A Z} \mathbf{T}_{\mathrm{m}}$, steady-state and time-resolved fluorescence revealed a decrease of emission and shortening of singlet excited state lifetime of both photosensitizers. The bimolecular rate constants of the electron transfer process $\left(\mathrm{k}_{\mathrm{q}}\right)$ were 4.0 and $1.7 \times 10^{9} \mathrm{M}^{-1} \mathrm{~s}^{-1}$ for $1-\mathrm{MN}$ and CHRY, respectively. This tendency is in agreement with the redox potential of the photosensitizer in the singlet excited state that points to $1-\mathrm{MN}$ as a better reductant than CHRY. Similar values of $\mathrm{k}_{\mathrm{q}}$ were obtained for $\mathbf{T}_{\mathrm{m}}<>\mathbf{A Z} \mathbf{T}_{\mathrm{m}}$ and $\mathbf{T}_{\mathrm{m}}<>\mathbf{T}_{\mathrm{m}}$, this is suggestive of a close value for the reduction potential of both substrates. In addition, these results suggest that the thymine moiety is relevant in the electron transfer process. 
Table 3.2. Oxidation potential in the singlet excited state $\left(\mathrm{E}_{\mathrm{ox}}^{\mathrm{D} *}\right)$ of the selected PS, and bimolecular rate constant $\left(\mathrm{k}_{\mathrm{q}}\right)$ for the quenching of the PS by $\mathbf{T}_{\mathrm{m}}<>\mathbf{T}_{\mathrm{m}}$ determined by time-resolved fluorescence.

\begin{tabular}{c|ccccc}
$\mathbf{P S}$ & $\begin{array}{c}\mathbf{E}_{\mathbf{o x}}^{\mathbf{D}} / \mathbf{V} \mathbf{v s .} \\
\mathbf{A g} / \mathbf{A g C l}\end{array}$ & $\begin{array}{c}\mathbf{E}_{\mathbf{o x}}^{\mathbf{D}} / \mathbf{V} \mathbf{v s .} \\
\mathbf{A g} / \mathbf{A g C l}\end{array}$ & $\begin{array}{c}\mathbf{E}^{*} / \mathbf{k c a l} \\
\mathbf{m o l}^{-1}\end{array}$ & $\boldsymbol{\tau} / \mathbf{n s}$ & $\mathbf{k}_{\mathbf{q}} / \mathbf{x} \mathbf{1 0} \mathbf{M}^{-1} \mathbf{s}^{-1}$ \\
\hline $1-\mathrm{MN}$ & $-2.5^{25}$ & 1.4 & 89.0 & 6.2 & 3.9 \\
$\mathrm{CHRY}$ & $-2.1^{24}$ & 1.3 & 79.3 & 11.9 & 1.1
\end{tabular}
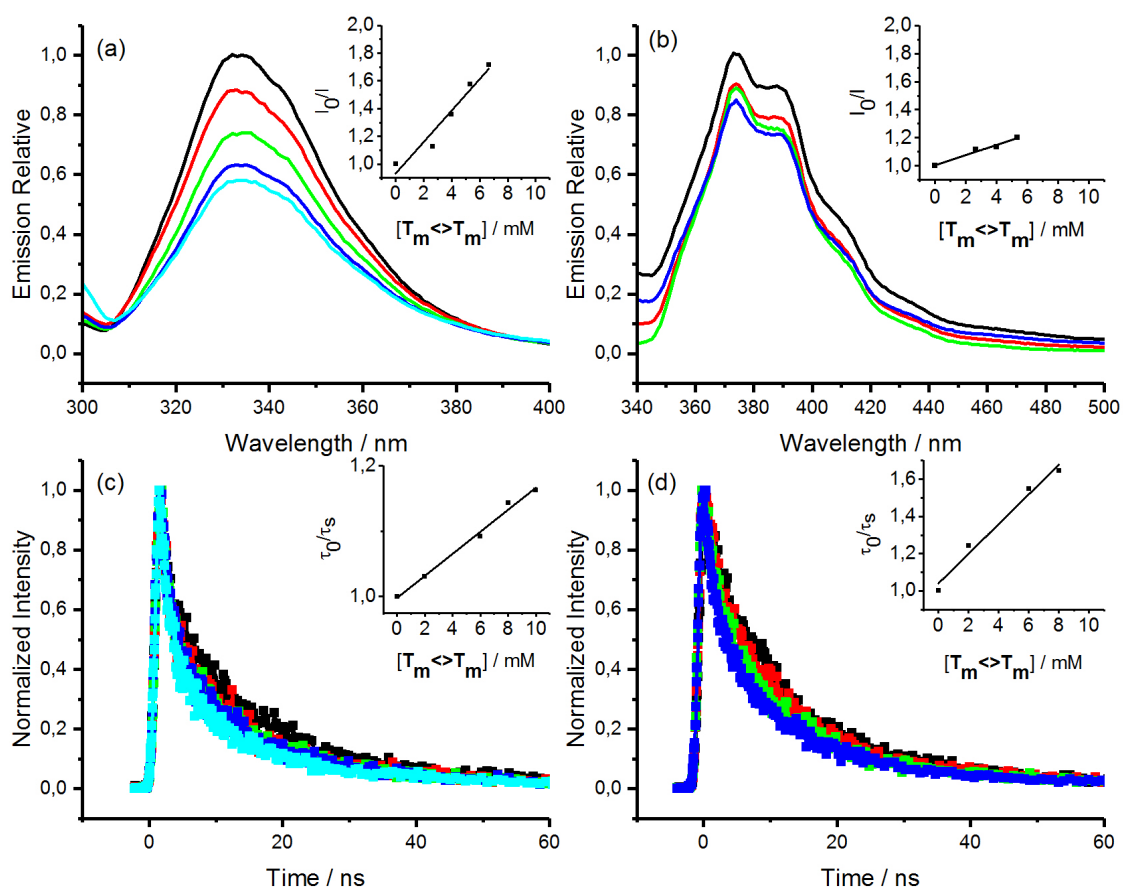

Figure 3.10. Fluorescence emission spectra (top) and kinetic traces (bottom) for $(\mathrm{a}, \mathrm{c}) 1-\mathrm{MN}\left(\lambda_{\text {exc }}=310 \mathrm{~nm}\right)$; (b, d) CHRY $\left(\lambda_{\text {exc }}=310 \mathrm{~nm}\right)$ in the presence of increasing amounts of $\mathbf{T}_{\mathrm{m}}<>\mathbf{T}_{\mathrm{m}}(0-7 \mathrm{mM})$. Inset: corresponding Stern-Volmer plot. 
In order to support that the electron transfer involved the thymine nucleobase, fluorescence experiments were performed for $\mathbf{D C H}<>\mathbf{A Z} \mathbf{T}_{\mathrm{m}}$ that only has the azaU moiety. In this context, DMA and CAR were used as photoreductants for the steadystate fluorescence experiments. The emission spectra of CAR and DMA suffered only weak changes when trans $\mathbf{D C H}<>\mathbf{A Z} \mathbf{T}_{\mathrm{m}}$ azetidine was added, which means that electron injection is not an efficient process (Figure 3.11).
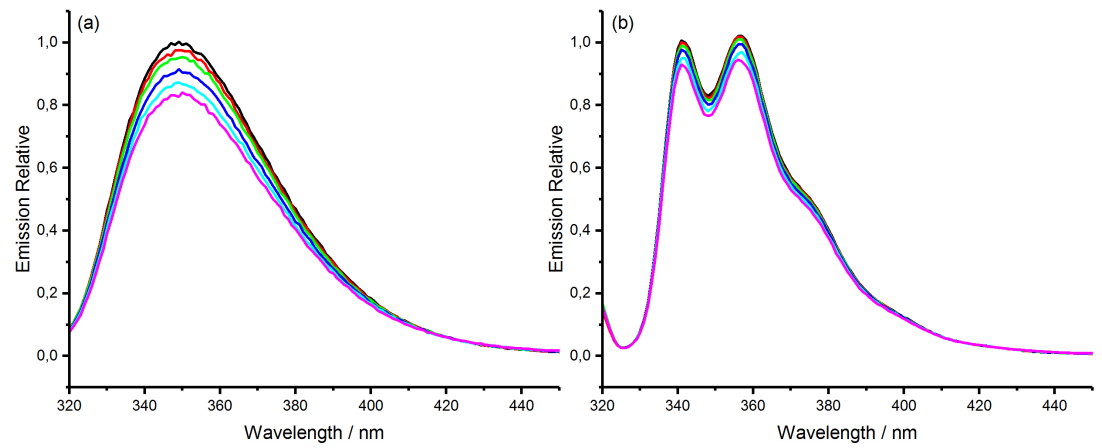

Figure 3.11. Fluorescence emission spectra for (a) DMA $\left(\lambda_{\text {exc }}=310 \mathrm{~nm}\right)$ and (b) CAR $\left(\boldsymbol{\lambda}_{\text {exc }}=310 \mathrm{~nm}\right)$ in the presence of increasing amounts of trans $\mathbf{D C H}<>\mathbf{A Z} \mathbf{T}_{\mathrm{m}}$.

\subsubsection{Electrochemical measurements}

As shown in the Rehm-Weller equation 3.3, oxidation and reduction potentials of the species involved in the photoinduced electron transfer are the essential factors that determine the feasibility of this process. In this context, the electrochemical properties of $\mathbf{T}_{\mathrm{m}}<>\mathrm{AZT}_{\mathrm{m}}$ and $\mathbf{T}_{\mathrm{m}}<>\mathbf{T}_{\mathrm{m}}$ were investigated by cyclic voltammetry. According to the electron injection, $\mathbf{T}_{\mathrm{m}}<>\mathbf{A} \mathbf{Z} \mathbf{T}_{\mathrm{m}}$ and $\mathbf{T}_{\mathrm{m}}<>\mathbf{T}_{\mathrm{m}}$ exhibited similar behavior with an irreversible wave appearing at very negative potential of ca. $-2.6 \mathrm{~V}$ vs. $\mathrm{Ag} / \mathrm{AgCl}$ (Figure 3.12). This value is compatible with that reported for the cis-syn cyclobutane dimers of 1,3-dimethythymine. ${ }^{31-32}$ Therefore, this result supports the similar values of $\mathrm{k}_{\mathrm{q}}$ obtained for the fluorescence experiments. 
Once obtained the $\mathrm{E}_{\text {red }}^{\mathrm{A}}$ value, $\Delta \mathrm{G}_{\mathrm{ct}}$ (without taking into account the coulombic interaction) was determinated in order to know if PET is an endergonic or exergonic process. Using the $\mathrm{E}_{\mathrm{Ox}}^{\mathrm{D}}$ values for TMB and CHRY photosensitizers, which are the best and worse electron donors, values of ca. $-13.3 \mathrm{kcal} / \mathrm{mol}$ and $11.9 \mathrm{kcal} / \mathrm{mol}$ were obtained, respectively. This agrees with a more efficient process for TMB than for CHRY with a better $\mathrm{k}_{\mathrm{q}}$ obtained by time-resolved fluorescence measurements.

$$
\Delta G_{c t}=23.06\left(E_{o x}^{D}-E_{r e d}^{A}\right)-E^{*}
$$

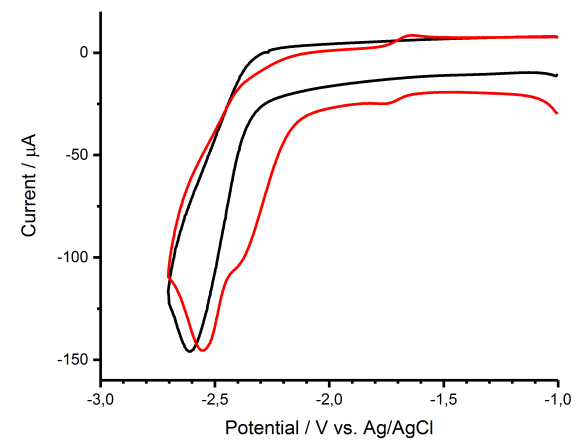

Figure 3.12. Cyclic voltammograms of $\mathbf{T}_{\mathbf{m}}<>\mathbf{A Z} \mathbf{T}_{\mathrm{m}}(2 \mathrm{mM}$, red line) and $\mathbf{T}_{\mathrm{m}}<>\mathbf{T}_{\mathrm{m}}\left(2 \mathrm{mM}\right.$, black line) in $\mathrm{N}_{2}$-purged DMF using $0.1 \mathrm{M} \mathrm{n}-\mathrm{Bu}_{4} \mathrm{NClO}_{4}$ as electrolyte. Scan rate: $0.1 \mathrm{Vs}^{-1}$.

\subsubsection{Photocycloreversion of $\mathrm{T}_{\mathrm{m}}<>\mathrm{AZT}_{\mathrm{m}}$}

Steady-state photolysis was performed to ensure that the fluorescence quenching resulted in the expected ring splitting reaction. For this purpose, TMPD was selected as photosensitizer on the basis of the above results. Thus, an acetonitrile solution of $\mathbf{T}_{\mathrm{m}}<>\mathbf{A Z T}_{\mathrm{m}}$ in the presence of TMPD was irradiated monochromatically at $350 \mathrm{~nm}$ to make sure that the light is selectively absorbed by TMPD, avoiding this way the possibility of direct photolysis. ${ }^{17-18}$ The sample was purged with nitrogen, irradiated for different times, and analyzed by HPLC to determine the amounts of obtained 
photoproduct and remaining $\mathbf{T}_{\mathrm{m}}<>\mathbf{A Z Z} \mathbf{T}_{\mathrm{m}}$ (Figure 3.13). Only one photoproduct was observed, which was assigned to compound $\mathbf{T}_{\mathrm{m}}-\mathbf{a z a} \mathbf{U}_{\mathrm{m}}$ by comparison with an authentic sample. This demonstrates that the electron transfer process results in a clean cycloreversion of the azetidine ring.
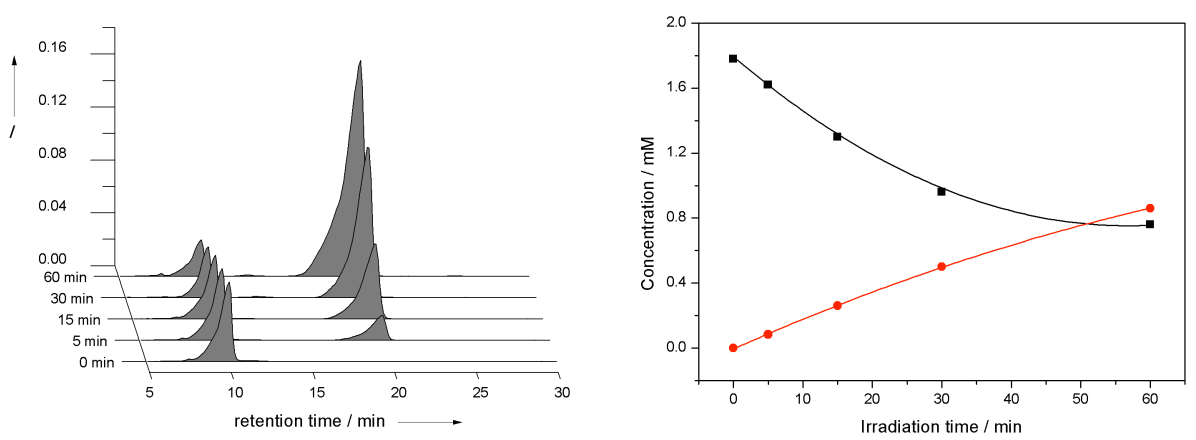

Figure 3.13. HPLC chromatogram (left) obtained after 0, 5, 15, 30, $60 \mathrm{~min}$ of irradiation of a mixture of $\mathbf{T}_{\mathbf{m}}<>\mathbf{A Z} \mathbf{T}_{\mathbf{m}}(2 \mathrm{mM})$ and TMPD $(4 \mathrm{mM})$ in acetonitrile with $350 \mathrm{~nm}$ light. Variation of the concentration of $\mathbf{T}_{\mathrm{m}}<>\mathbf{A Z} \mathbf{T}_{\mathrm{m}}$ (square) and repaired $\mathbf{T}_{\mathrm{m}}-\mathbf{a z a} \mathbf{U}_{\mathrm{m}}$ (circle) with the irradiation time.

In addition, experimental evidence in support of the electron transfer mechanism was obtained by UV/Vis spectrophotometry. As shown in Figure 3.14, irradiation of TMPD in the presence of $\mathbf{T}_{\mathrm{m}}<>\mathbf{A Z} \mathbf{T}_{\mathrm{m}}$ resulted in the formation of a species absorbing between 500 and $650 \mathrm{~nm}$, which is coincident in shape and position with the visible band of TMPD radical cation. ${ }^{33}$ However, it is important to mention that the observed species does not correspond to the "in cage" $\mathrm{TMPD}^{\bullet+}$ responsible for $\mathbf{T}_{\mathrm{m}}<>\mathbf{A Z} \mathbf{T}_{\mathrm{m}}$ cycloreversion but to the longer-lived free radical cation escaped from the solvent cage, which is present at very low concentration (ca. $5 \times 10^{-6} \mathrm{M}$ ). 


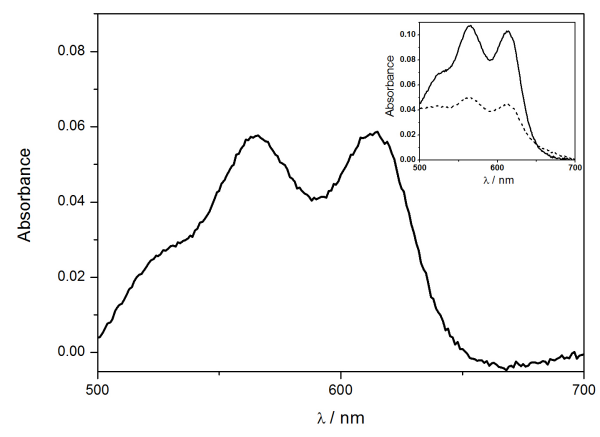

Figure 3.14. UV absorption spectra of TMPD $^{\bullet+}\left(5 \times 10^{-6} \mathrm{M}\right)$ formed by irradiation of TMPD (4 mM) in the presence of $\mathbf{T}_{\mathrm{m}}<>\mathbf{A Z} \mathbf{T}_{\mathrm{m}}(2 \mathrm{mM})$ for $60 \mathrm{~min}$ at $350 \mathrm{~nm}$. For a better clarity, absorption from control experiment (TMPD irradiated alone) was subtracted. Inset: UV absorption spectra of TMPD $^{\bullet+}$ : TMPD irradiated in presence of $\mathbf{T}_{\mathbf{m}}<>\mathbf{A Z} \mathbf{T}_{\mathbf{m}}$ (line) and alone (dash line) for $60 \mathrm{~min}$ at $350 \mathrm{~nm}$.

\subsubsection{Quantum-chemistry determination of the photoreductive properties of T<>AZT}

This study was performed in collaboration with the research group of Dr. RocaSanjúan (University of Valencia). To gain insight into the energetics involved in the injection of one electron in the azetidine and cyclobutane derivatives, the electron affinities (EAs) were computed for the $N$-demethylated compounds $\mathbf{T}<>$ AZT $\mathbf{T}$ and $\mathbf{T}<>\mathbf{T}$ with the density functional theory (DFT) method, the M06-2X functional and the $6-31++G(\mathrm{~d}, \mathrm{p})$ basis set. Benchmark calculations on the thymine nucleobase compared with high-level complete-active-space self-consistent field second-order perturbation theory (CASPT2) results indicate an accurate performance of the DFT/M062X method for the adiabatic EAs (AEAs). Table 3.3 compiles the computed gas phase and acetonitrile solution values, which also allows an estimation of the solvent effect. As expected, solvation has an important influence on the EA values because it stabi- 
lizes to a higher extent the ionic states as compared to the neutral state. Moreover, Table 3.3 shows that EAs of methylated and demethylated compounds of $\mathbf{T}<>\mathbf{A Z T}$ differ slightly, thus theoretical studies with demethylated give us reliable results as for methylated compounds. According to $\mathbf{T}<>\mathbf{T}$, EAs are very similar to $\mathbf{T}<>\mathbf{A Z T}$, which agrees with the comparable experimental quenching rates $\left(k_{q}\right)$ for both compounds.

Table 3.3. Adiabatic electron affinities (AEAs) in $\mathrm{eV}\left(\mathrm{kcal} \mathrm{mol}^{-1}\right.$ within parentheses) for $\mathbf{T}<>\mathbf{A Z T}, \mathbf{T}_{\mathrm{m}}<>\mathbf{A Z T}_{\mathrm{m}}$ and $\mathbf{T}<>\mathbf{T}$ in the gas phase and in acetonitrile computed with the DFT/M06-2X method and the 6-31++G(d,p) basis set.

\begin{tabular}{lc}
\hline & AEA \\
\hline $\mathbf{T}<>$ AZT & \\
Gas phase & $0.50(11.6)$ \\
Acetonitrile & $2.16(49.9)$ \\
$\mathbf{T}_{\mathrm{m}}<>\mathbf{A Z T}_{\mathbf{m}}$ & \\
Gas phase & $0.30(9.0)$ \\
Acetonitrile & $2.09(48.2)$ \\
$\mathbf{T}<>\mathbf{T}$ & \\
Gas phase & $0.31(7.1)$ \\
Acetonitrile & $2.00(46.1)$ \\
\hline
\end{tabular}

\subsubsection{Decomposition mechanisms of $\mathrm{T}<>\mathrm{AZT}$ radical anion}

Injection of one electron into the azetidine $\mathbf{T}<>\mathbf{A Z T}$ produces the rupture of the $\mathrm{C}-\mathrm{C}$ bond due to the fact that the anion of the four-membered ring is not a stable structure (Scheme 3.8). The extra electron from the photosensitizer is partially located in the $\sigma^{*}$ orbital of the $\mathrm{C}-\mathrm{C}$ bond thus weakening this bond, which breaks. For $\mathbf{T}<>\mathbf{T}$, previous studies ${ }^{34}$ and the current work also indicate a more favorable open structure for the anion. 


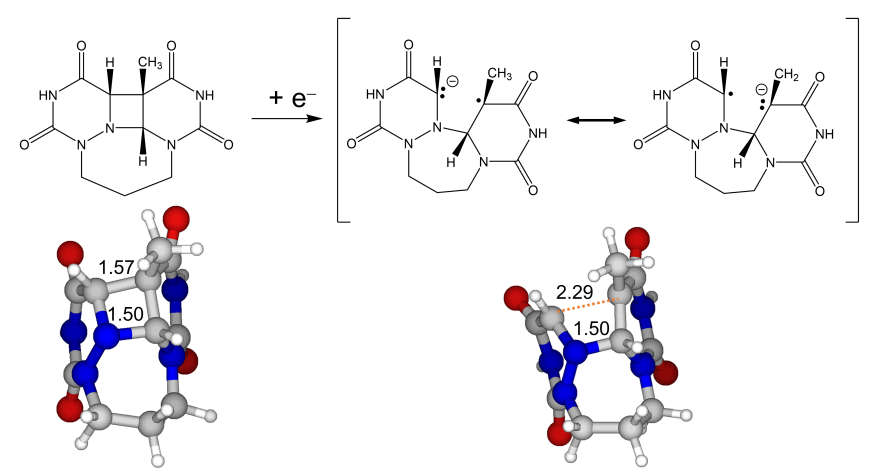

Scheme 3.8. Chemical structures (top) and geometries (bottom) of the neutral $\mathbf{T}_{\mathrm{m}}<>\mathbf{A Z} \mathbf{T}_{\mathrm{m}}$ (left) and its radical anion $\mathbf{T}_{\mathrm{m}}<>\mathbf{A Z} \mathbf{T}_{\mathrm{m}}{ }^{\bullet-}$ (right). Relevant bond distances for the reaction are shown in $\AA$.

Evolution of the system towards recovery of the thymine and 6-azauracil molecules has also been studied with appropriate reaction path computational strategies showing an electronic energy barrier height of around $13 \mathrm{kcal} \mathrm{mol}^{-1}$ (Scheme 3.9). Due to technical difficulties to accurately determine the transition state (TS) structure, an approximated procedure has been used. This approach, based on minimum energy path (MEP) and linear interpolation of internal coordinates (LIIC) calculations, gives rise to a connected path between reactant and product and provides an upper-bound value for the barrier. Therefore, the exact energy barrier height shall be expected at slightly lower energies in the range of $10-13 \mathrm{kcal} \mathrm{mol}^{-1}$. 


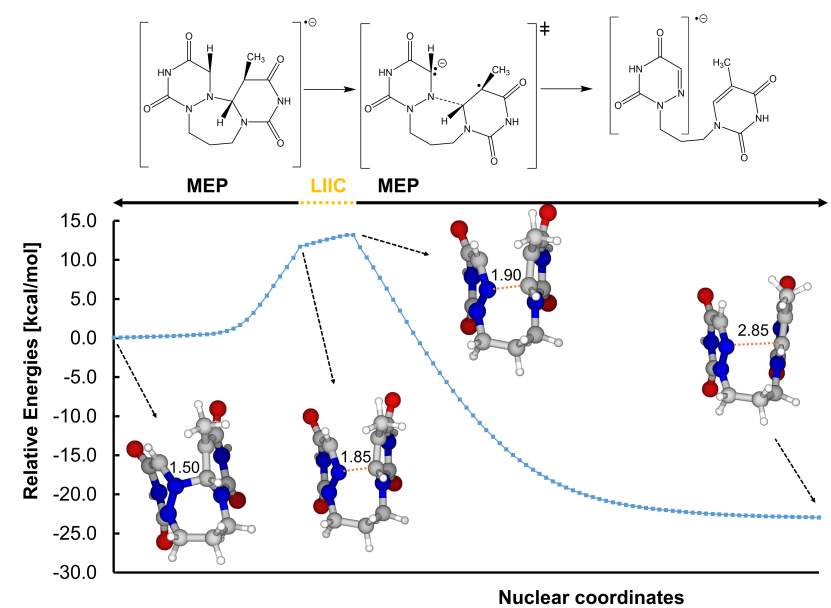

Scheme 3.9. Chemical structure of relevant points (top) and energy profile (bottom) for the C-N and C-C bond breakings of $\mathbf{T}_{\mathrm{m}}<>\mathrm{AZT}_{\mathrm{m}}{ }^{\bullet-}$ obtained with MEP and LIIC computational strategies. Relevant bond distances for the reaction are shown in $\AA$.

Regarding the electronic-structure properties, analysis of the spin density obtained with the complete-active space self-consistent field (CASSCF) method indicates a delocalization of the extra electron over the two carbon atoms which breaks at the reactant side of the cycloreversion mechanism (Figure 3.15). Once the two monomers are regenerated, the unpaired electron is localized in 6-azauracil. Therefore, these data showed that the reductive mechanism of $\mathbf{T}_{\mathrm{m}}<>\mathbf{A Z} \mathbf{T}_{\mathrm{m}}$ occurs via pathway $\mathrm{b}$ in the Scheme 3.7. 

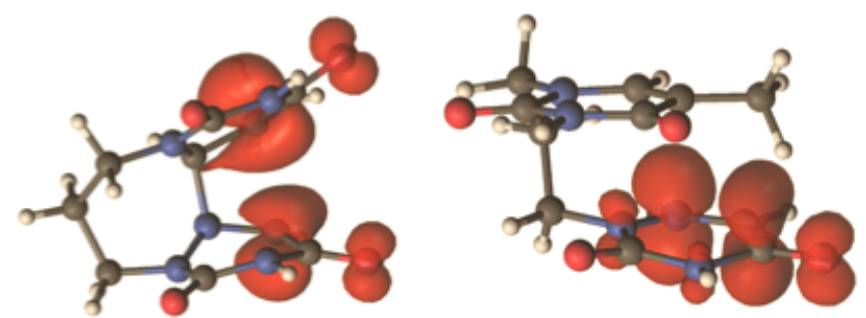

Figure 3.15. CASSCF spin-density representations for the $\mathbf{T}<>$ AZT radical anion systems at the reactant (left) and product (right) geometries, respectively. Red $=$ excess of spin density, green $=$ defect of spin density.

Globally, the in-vacuo mechanism described above implies energy barriers lower than $13 \mathrm{kcal} \mathrm{mol}^{-1}$ for the radical anion system. The cycloreversion process is clearly exergonic for $\mathbf{T}<>\mathbf{A Z T} \mathbf{T}^{\bullet-}$. To further improve the description, we have focused on the relevant points for the present mechanism (reactants, TSs and products) and we have determined several thermochemical magnitudes using the highly accurate CASPT2 method. In particular, the activation energy $\left(\mathrm{E}^{\#}\right)$ and thermodynamics $(\Delta \mathrm{E})$, the zero-point vibrational energy (ZPVE) corrected values $\left(\Delta \mathrm{E}_{0}^{\#}\right.$ and $\left.\Delta \mathrm{E}_{0}\right)$ and the Gibbs free energies ( $\Delta G^{\#}$ and $\Delta G$ ) have been computed at both gas and solution phases. Results are compiled in Table 3.4. As it can be seen, the CASPT2 method confirms the relatively low DFT energy barrier heights and the highly exergonic character of the $\mathbf{T}<>\mathbf{A Z} \mathbf{T}^{\bullet-}$ ring opening reaction. Regarding the solvent effects, in contrast to the redox properties determined in the previous section, no significant changes are observed for the $\mathbf{T}<>\mathbf{A Z T} \mathbf{T}^{\bullet-}$ cycloreversion mechanisms. This is due to the fact that along the ring opening reactions the total charge is preserved. The ZPVE correction gives rise to lower $\Delta \mathrm{Es}$ as a consequence of the bond breakings occurring along the reactions. The same behavior is observed for $\Delta \mathrm{G}$, which reflects the entropy increase in the decomposition process. 
Table 3.4. Computed energy differences between products and the reactants $(\Delta \mathrm{E}$, $\Delta \mathrm{E}_{0}$ and $\left.\Delta \mathrm{G}\right)$ and activation energies $\left(\Delta \mathrm{E}^{\ddagger}, \Delta \mathrm{E}_{0}{ }^{\ddagger}\right.$ and $\left.\Delta \mathrm{G}^{\ddagger}\right)$ for the cycloreversion of the $\mathbf{T}<>$ AZT radical anion. Energies in $\mathrm{kcal}^{\mathrm{mol}}{ }^{-1}$.

\begin{tabular}{|c|c|c|}
\hline \multirow[t]{2}{*}{ Methodology } & \multicolumn{2}{|c|}{$\mathrm{AZT}^{\circ}$} \\
\hline & $\Delta \mathrm{E}^{[\mathrm{a}]}$ & $\Delta \mathrm{E}^{\ddagger}$ \\
\hline M06-2X & $-29.07^{[b]}$ & $11.69-13.20^{[\mathrm{b}]}$ \\
\hline CASPT2//M06-2X & $-25.16^{[b]}$ & $11.88-12.85^{[\mathrm{b}]}$ \\
\hline \multirow[t]{2}{*}{ PCM-M06-2X } & $-29.20[c]$ & $11.22-12.51[\mathrm{c}]$ \\
\hline & $\Delta \mathrm{E}_{0}$ & $\Delta \mathrm{E}_{0}^{\ddagger}$ \\
\hline M06-2X & $-29.87[\mathrm{~d}]$ & $-[\mathrm{e}]$ \\
\hline \multirow[t]{2}{*}{ CASPT2//M06-2X } & $-25.96[d]$ & $-[\mathrm{e}]$ \\
\hline & $\Delta \mathrm{G}$ & $\Delta \mathrm{G}^{\neq}$ \\
\hline M06-2X & $-30,99[\mathrm{ff}]$ & $-[\mathrm{e}]$ \\
\hline CASPT2//M06-2X & $-27.08^{[f]}$ & $-[\mathrm{e}]$ \\
\hline
\end{tabular}

[a] Energies related to the lowest-energy conformer of the product (see text). [b] Electronic energy (gas phase). [c] Electronic energy including solvent effects (acetonitrile). [d] Electronic energy with ZPVE corrections (gas phase). [e] No TS found. [f] Electronic energy with thermal and entropic contributions at $298.15 \mathrm{~K}$ and $1 \mathrm{~atm}$ (gas phase).

\subsection{Conclusions}

The present study has clearly demonstrated for the first time that the photoinduced injection of one electron into a dimeric azetidine derived from thymine leads to a clean cycloreversion and therefore to the "repair" of the nucleobases. In a first stage, the electron donation capabilities of the azetidine heterocycle have been assessed using photoreductant singlet excited state. In this context, comparison of the bimolecular rate constants obtained for $\mathbf{T}_{\mathrm{m}}<>\mathbf{A Z} \mathbf{T}_{\mathrm{m}}$ and $\mathbf{T}_{\mathrm{m}}<>\mathbf{T}_{\mathrm{m}}$ revealed that the presence of the nitrogen atom in the ring does not affect the reduction process; however the 
Chapter 3: Photoreduction of an Azetidine Model of the (6-4) Photoproduct

thymine moiety is essential for the occurrence of the electron transfer, which agrees with the lack of fluorescence quenching in the case of $\mathbf{D C H}<>\mathbf{A Z T}_{\mathbf{m}}$. The electrochemical experiments support these redox properties as close reduction potentials have been registered. This fact has also been corroborated by DFT quantum chemistry, which has determined similar electron affinities. Concerning the $\mathbf{T}_{\mathrm{m}}<>\mathbf{A Z Z} \mathbf{T}_{\mathrm{m}}$ radical anion, the injection of an electron in the heterocycle provokes the direct cleavage of the interbase $\mathrm{C}-\mathrm{C}$ bond at $\mathrm{C} 5$, followed by splitting of the $\mathrm{C}-\mathrm{N}$ at position 6 , which involves a low energy barrier of ca. $13 \mathrm{kcal} \mathrm{mol}^{-1}$, and finally leaves a radical anion centered on the 6-azauracil moiety. Altogether the obtained results are relevant to understand the process involved in of (6-4) photolyase and support the feasibility of the mechanistic pathway involving reductive splitting of an azetidine intermediate.

\subsection{Experimental section}

\subsubsection{Synthesis}<smiles>Cc1cn(C)c(=O)n(C)c1=O</smiles>

3,5-Dimethylpyrimidine-2,4(1H,3H)-dione (1): A solution of thymine $(4.0 \mathrm{~g}, 31.7 \mathrm{mmol})$ in acetic anhydride $(100 \mathrm{~mL})$ was refluxed overnight. The reaction mixture was then cooled to $\mathrm{rt}$ and dried under vacuum. The crude product was repeatedly treated with toluene and concentrated under vacuum to give the acetyl derivative (4.8 g, 90\%). The resultant acetyl derivative (4.8 g, 29.6 mmol) was added to a suspension of $\mathrm{NaH}(0.75 \mathrm{~g}, 31.4 \mathrm{mmol})$ in anhydrous acetonitrile $(100 \mathrm{~mL})$. After stirring for 1 hour at $\mathrm{rt}$, the reaction mixture was treated with iodomethane $(2.1 \mathrm{~mL}, 34.2 \mathrm{mmol})$ and stirred for $12 \mathrm{~h}$ at $70{ }^{\circ} \mathrm{C}$. The solvent was removed, and the residue was purified by flash chromatography (silica gel, 98\% dichloromethane/methanol) to give $\mathbf{1}(2.8 \mathrm{~g}, 62 \%)$ as a white solid powder. ${ }^{1} \mathbf{H}$ NMR $\left(300 \mathrm{MHz}, \mathrm{CDCl}_{3}\right) \delta 10.36$ (br s, $\left.1 \mathrm{H}\right), 7.06(\mathrm{~d}, J=4.5 \mathrm{~Hz}, 1 \mathrm{H}), 3.34$ (s, 3H), 1.93 (s, 3H). ${ }^{13} \mathbf{C}$ NMR $\left(75 \mathrm{MHz}, \mathrm{CDCl}_{3}\right) \delta 164.7$ (C), $153.6(\mathrm{C}), 134.4(\mathrm{CH}), 110.4(\mathrm{C}), 27.4$ 
Chapter 3: Photoreduction of an Azetidine Model of the (6-4) Photoproduct

$\left(\mathrm{CH}_{3}\right), 12.7\left(\mathrm{CH}_{3}\right)$. HRMS (ESI): $m /$ z calcd for $\mathrm{C}_{6} \mathrm{H}_{9} \mathrm{~N}_{2} \mathrm{O}_{2}[\mathrm{M}+\mathrm{H}]^{+}$141.0664, found 141.0660 .

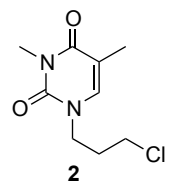

1-(3-Chloropropyl)-3,5-dimethylpyrimidine-2,4-(1H,3H)-dione (2):

N3-methylthymine $1(2.5 \mathrm{~g}, 17.8 \mathrm{mmol})$ was added to a suspension of mixture was stirred at room temperature for 1 hour followed by the addition of 1 bromo-3-chloropropane $(1.9 \mathrm{~mL}, 19.62 \mathrm{mmol})$, and stirred for $12 \mathrm{~h}$ at $70{ }^{\circ} \mathrm{C}$. The solvent was removed, and the residue was purified by flash chromatography (silica gel, 98\% dichloromethane/methanol) to give the product $\mathbf{2}(2.8 \mathrm{~g}, 60 \%)$ as a yellow oil. ${ }^{1} \mathbf{H}$ NMR $\left(300 \mathrm{MHz}, \mathrm{CDCl}_{3}\right) \delta 7.06(\mathrm{~s}, 1 \mathrm{H}), 3.92(\mathrm{t}, J=6.6 \mathrm{~Hz}, 2 \mathrm{H}), 3.60(\mathrm{t}, J=6.0 \mathrm{~Hz}$, $2 \mathrm{H}), 3.37(\mathrm{~s}, 3 \mathrm{H}), 2.25-2.13(\mathrm{~m}, 2 \mathrm{H}), 1.96(\mathrm{~s}, 3 \mathrm{H}) .{ }^{13} \mathbf{C} \mathbf{N M R}\left(75 \mathrm{MHz}, \mathrm{CDCl}_{3}\right) \delta$ $163.8(\mathrm{C}), 151.4(\mathrm{C}), 138.3(\mathrm{CH}), 109.6(\mathrm{C}), 46.9\left(\mathrm{CH}_{2}\right), 41.4\left(\mathrm{CH}_{2}\right), 30.9\left(\mathrm{CH}_{2}\right), 27.8$ $\left(\mathrm{CH}_{3}\right), 12.8\left(\mathrm{CH}_{3}\right)$. HRMS (ESI): $m / z$ calcd for $\mathrm{C}_{9} \mathrm{H}_{14} \mathrm{~N}_{2} \mathrm{O}_{2} \mathrm{Cl}[\mathrm{M}+\mathrm{H}]^{+}$217.0744, found 217.0744.

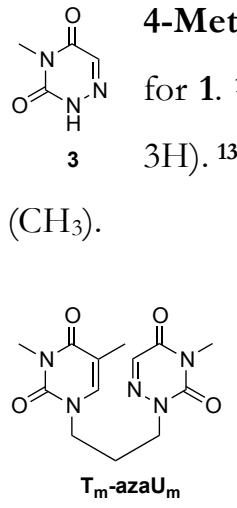

-Methyl-1,2,4-triazine-3,5(2H,4H)-dione (3) was prepared as described for 1. ${ }^{1} \mathbf{H}$ NMR (300 MHz, $\left.\mathrm{CDCl}_{3}\right) \delta 10.39$ (br s, $\left.1 \mathrm{H}\right), 7.37$ (s, 1H), 3.28 (s, H). ${ }^{13} \mathbf{C}$ NMR $\left(75 \mathrm{MHz}, \mathrm{CDCl}_{3}\right) \delta 156.2(\mathrm{C}), 149.9(\mathrm{C}), 135.3(\mathrm{CH}), 26.3$

$T_{\mathrm{m}}$-aza

2-[3-(3,5-Dimethyl-2,4-dioxo-3,4-dihydropyrimidin-1(2H)yl)propyl]-4-methyl-1,2,4-triazine-3,5(2H,4H)-dione $\quad\left(\mathrm{T}_{\mathrm{m}}-\right.$ $\left.\mathbf{a z a U}_{\mathrm{m}}\right)$ : Potassium carbonate $(2.4 \mathrm{~g}, 17.7 \mathrm{mmol})$ was added to a solution of $2(2.8 \mathrm{~g}, 13.0 \mathrm{mmol})$ and $\mathbf{3}(1.5 \mathrm{~g}, 11.8 \mathrm{mmol})$ in dry acetonitrile $(100 \mathrm{~mL})$. The resulting solution was stirred 18 hours at $80{ }^{\circ} \mathrm{C}$. The solvent was removed under reduced pressure, and the crude product was washed by hot methanol to give $\mathbf{T}_{\mathrm{m}}$-azaU $\mathbf{U}_{\mathrm{m}}(2.5 \mathrm{~g}, 70 \%)$ as a beige solid. ${ }^{1} \mathbf{H}$ NMR $(300 \mathrm{MHz}$, $\left.\mathrm{CDCl}_{3}\right) \delta 7.41(\mathrm{~s}, 1 \mathrm{H}), 7.05(\mathrm{~s}, 1 \mathrm{H}), 4.05(\mathrm{t}, J=6.7 \mathrm{~Hz}, 2 \mathrm{H}), 3.80(\mathrm{t}, J=7.0 \mathrm{~Hz}, 2 \mathrm{H})$, 3.34 (s, 6H), $2.27-2.09$ (m, 2H), $1.94(\mathrm{~s}, 3 \mathrm{H}) ;{ }^{13} \mathbf{C}$ NMR (75 MHz, $\left.\mathrm{CDCl}_{3}\right) \delta 163.7$ 
Chapter 3: Photoreduction of an Azetidine Model of the (6-4) Photoproduct

(C), $155.8(\mathrm{C}), 151.4(\mathrm{C}), 148.8(\mathrm{C}), 137.8(\mathrm{CH}), 134.1(\mathrm{CH}), 109.8(\mathrm{C}), 48.6\left(\mathrm{CH}_{2}\right)$, $46.6\left(\mathrm{CH}_{2}\right), 27.8\left(\mathrm{CH}_{3}\right), 27.5\left(\mathrm{CH}_{2}\right), 26.8\left(\mathrm{CH}_{3}\right), 12.9\left(\mathrm{CH}_{3}\right)$. HRMS (ESI): $m / z$ calcd for $\mathrm{C}_{13} \mathrm{H}_{18} \mathrm{~N}_{5} \mathrm{O}_{4}[\mathrm{M}+\mathrm{H}]^{+} 308.1359$, found 308.1350 .

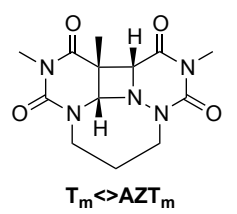

Azetidine photoproduct $\left(\mathbf{T}_{\mathrm{m}}<>\mathbf{A Z} \mathbf{T}_{\mathrm{m}}\right)$ : In a Pyrex vessel $(\boldsymbol{\lambda}>290$ $\mathrm{nm})$, acetone solutions of $\mathbf{T}_{\mathrm{m}}-\mathbf{a z a} \mathbf{U}_{\mathrm{m}}\left(1 \times 10^{-2} \mathrm{M}\right)$ were irradiated under nitrogen atmosphere for 1 hour using a medium pressure mercury lamp $(125 \mathrm{~W})$. After that time, no traces of the substrate were detected by TLC using hexane/ethyl acetate (1:3) as eluent. The irradiated solution was evaporated to dryness. The residue was washed with cold ethanol and dried in vacuo to give $\mathbf{T}_{\mathbf{m}}<>\mathbf{A Z T}_{\mathbf{m}}(75 \%)$ as a white solid. ${ }^{1} \mathbf{H} \mathbf{~ N M R}\left(300 \mathrm{MHz}, \mathrm{CDCl}_{3}\right) \delta 4.79$ (s, 1H), 4.57 (s, 1H), 4.49 - $4.41(\mathrm{~m}, 2 \mathrm{H}), 3.14(\mathrm{~s}, 3 \mathrm{H}), 3.12(\mathrm{~s}, 3 \mathrm{H}), 2.97-2.82(\mathrm{~m}, 2 \mathrm{H})$, $2.45-2.26(\mathrm{~m}, 1 \mathrm{H}), 1.88(\mathrm{~s}, 3 \mathrm{H}), 1.75-1.55(\mathrm{~m}, 1 \mathrm{H}) .{ }^{13} \mathbf{C} \mathbf{~ N M R}\left(75 \mathrm{MHz}, \mathrm{CDCl}_{3}\right) \delta$ 165.8 (C), 164.5 (C), 150.9 (C), 149.9 (C), $80.5(\mathrm{CH}), 67.2(\mathrm{CH}), 49.1\left(\mathrm{CH}_{2}\right), 48.5$ $\left(\mathrm{CH}_{2}\right), 45.2(\mathrm{C}), 28.4\left(\mathrm{CH}_{3}\right), 26.8\left(\mathrm{CH}_{3}\right), 23.3\left(\mathrm{CH}_{2}\right), 22.5\left(\mathrm{CH}_{3}\right)$. HRMS (ESI): $\mathrm{m} / \mathrm{z}$ calcd for $\mathrm{C}_{13} \mathrm{H}_{17} \mathrm{~N}_{5} \mathrm{O}_{4} \mathrm{Na}[\mathrm{M}+\mathrm{Na}]^{+} 330.1178$, found 330.1180 .

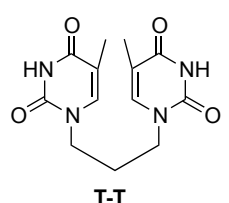

T-T

1,1'-(propane-1,3-diyl)bis(5-methylpyrimidine-2,4(1H,3H)dione) (T-T): O,O'-Bis(trimethylsilyl)-thymine $(1.0 \mathrm{~g}, 3.86 \mathrm{mmol})$ was added to a solution of 1,3-dibromopropane $(0.38 \mathrm{~g}, 1.68 \mathrm{mmol})$ in $6 \mathrm{~mL}$ of anhydrous DMF. The solution was heated to $170^{\circ} \mathrm{C}$ and stirred overnight. The reaction was cooled to $0{ }^{\circ} \mathrm{C}$ and $2 \mathrm{~mL}$ of water was added to the reaction mass to precipitate the product. The precipitated was stirred for $15 \mathrm{~min}$ at $0{ }^{\circ} \mathrm{C}$. The solids were filtered off, washed with $20 \mathrm{~mL}$ of chloroform/methanol (1:1) and dried in vacuo to give compound T-T $(0.9 \mathrm{~g}, 83 \%)$ as a white solid. ${ }^{\mathbf{1}} \mathbf{H}$ NMR (300 MHz, DMSO-d $\left.d_{6}\right) 11.20(\mathrm{~s}, 2 \mathrm{H}), 7.51(\mathrm{~s}, 2 \mathrm{H}), 3.64(\mathrm{t}, J=6.9 \mathrm{~Hz}, 4 \mathrm{H}), 2.05-1.79(\mathrm{~m}$, 2H). ${ }^{13} \mathrm{C}$ NMR (75 MHz, DMSO-d $d_{6} \delta 164.2(2 \mathrm{C}), 150.9(2 \mathrm{C}), 141.2(2 \mathrm{CH}), 108.5$ (2C), $44.8\left(2 \mathrm{CH}_{2}\right), 27.8\left(\mathrm{CH}_{2}\right), 11.9\left(2 \mathrm{CH}_{3}\right)$. 
Chapter 3: Photoreduction of an Azetidine Model of the (6-4) Photoproduct

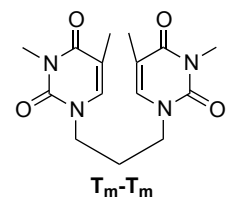

1,1'-(Propane-1,3-diyl)bis(3,5-dimethylpyrimidine-2,4(1H,3H)-

dione) $\left(\mathbf{T}_{\mathrm{m}}-\mathbf{T}_{\mathrm{m}}\right)$ : $\mathbf{T}-\mathbf{T}(0.9 \mathrm{~g}, 3.07 \mathrm{mmol})$ was added to a suspension of $\mathrm{NaH}(0.15 \mathrm{~g}, 6.14 \mathrm{mmol})$ in anhydrous DMF $(10 \mathrm{~mL})$. After stirring for 1 hour at room temperature, the reaction mixture was treated with iodomethane $(0.4 \mathrm{~mL}, 6.14 \mathrm{mmol})$ and stirred overnight at $\mathrm{rt}$. The reaction was cooled to $0{ }^{\circ} \mathrm{C}$ and the precipitated formed was filtered off, washed with $10 \mathrm{~mL}$ of water and dried in vacuo to give compound $\mathbf{T}_{\mathbf{m}}-\mathbf{T}_{\mathbf{m}}(0.9 \mathrm{~g}, 90 \%)$ as a white solid. ${ }^{1} \mathbf{H}$ NMR $\left(300 \mathrm{MHz}, \mathrm{CDCl}_{3}\right) \delta 6.99$ (s, 2H), $3.73(\mathrm{t}, J=7.0 \mathrm{~Hz}, 4 \mathrm{H}), 3.29$ (s, 6H), $2.06-$ $2.00(\mathrm{~m}, J=5.3 \mathrm{~Hz}, 2 \mathrm{H}), 1.87$ (s, 6H). ${ }^{13} \mathrm{C} \mathbf{~ N M R}\left(75 \mathrm{MHz}, \mathrm{CDCl}_{3}\right): 163.8(2 \mathrm{C}), 151.7$ (2C), $138.0(2 \mathrm{CH}), 110.2(2 \mathrm{C}), 46.8\left(2 \mathrm{CH}_{2}\right), 28.6\left(\mathrm{CH}_{2}\right), 28.0\left(2 \mathrm{CH}_{3}\right), 13.0\left(2 \mathrm{CH}_{3}\right)$.

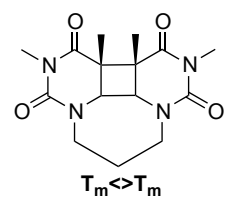

Cyclobutane photoproduct $\left(\mathbf{T}_{\mathrm{m}}<>\mathbf{T}_{\mathrm{m}}\right)$ : An acetone solution of $\mathbf{T}_{\mathrm{m}}-\mathbf{T}_{\mathrm{m}}\left(1 \times 10^{-2} \mathrm{M}\right)$ was irradiated in a Pyrex vessel $(\lambda>290 \mathrm{~nm})$ under nitrogen atmosphere for 2 hours using a medium pressure mercury lamp $(125 \mathrm{~W})$. After that time, no traces of the substrate were detected by TLC using hexane/ethyl acetate (1:3) as eluent. The irradiated solution was evaporated to dryness. The residue was washed with cold ethanol and dried in vacuo to give $\mathbf{T}_{\mathbf{m}}<>\mathbf{T}_{\mathrm{m}}(0.6,70 \%)$ as a white solid. ${ }^{1} \mathbf{H}$ NMR $\left(300 \mathrm{MHz}, \mathrm{CDCl}_{3}\right) \delta 4.48$ - $4.45(\mathrm{~m}, 1 \mathrm{H}), 4.44-4.40(\mathrm{~m}, 1 \mathrm{H}), 3.77(\mathrm{~s}, 2 \mathrm{H}), 3.13$ (s, 6H), $2.74-2.66(\mathrm{~m}, 2 \mathrm{H})$, $2.43-2.19(\mathrm{~m}, 1 \mathrm{H}), 1.65-1.63(\mathrm{~m}, 1 \mathrm{H}), 1.58(\mathrm{~s}, 6 \mathrm{H}) .{ }^{13} \mathbf{C} \mathbf{~ N M R}\left(75 \mathrm{MHz}, \mathrm{CDCl}_{3}\right) \delta$ $168.9(2 \mathrm{C}), 151.3(2 \mathrm{C}), 59.9(2 \mathrm{CH}), 48.9\left(2 \mathrm{CH}_{2}\right), 45.8(2 \mathrm{C}), 28.1\left(2 \mathrm{CH}_{3}\right), 23.8\left(\mathrm{CH}_{2}\right)$, $21.2\left(2 \mathrm{CH}_{3}\right)$.

$\begin{array}{ll}U_{N^{-}} & \text {1,3-Dimethyl-6-azauracil (4): 6-azauracil }(1.0 \mathrm{~g}, 8.84 \mathrm{mmol}) \text { was added to a } \\ N_{4} & \text { After stirring for } 1 \text { hour at } \mathrm{rt} \text {, the reaction mixture was treated with iodome- }\end{array}$ thane $(1.2 \mathrm{~mL}, 19.45 \mathrm{mmol})$ and stirred for 16 hours. The solvent was removed, and the residue was purified by flash chromatography (silica gel, 100\% dichloromethane) to give $4(0.8 \mathrm{~g}, 70 \%)$ as a white solid powder. ${ }^{1} \mathbf{H}$ NMR $\left(300 \mathrm{MHz}, \mathrm{CDCl}_{3}\right) \delta 7.36$ (s, 
1H), 3.63 (s, 3H), 3.33 ppm (s, 3H). ${ }^{13} \mathbf{C}$ NMR (75 MHz, $\left.\mathrm{CDCl}_{3}\right) \delta 156.4(\mathrm{C}), 149.1$ (C), $133.9(\mathrm{CH}), 39.8\left(\mathrm{CH}_{3}\right), 27.0 \mathrm{ppm}\left(\mathrm{CH}_{3}\right)$.

Cyclohexene-1,3-dimethyl-6-azauracil $\left(\mathbf{D C H}<>\mathbf{A Z T}_{\mathrm{m}}\right)$. A solution of 4 (0.5 g, $3.54 \mathrm{mmol}), 10 \mathrm{~mL}$ of acetone and $2.5 \mathrm{~mL}$ of cyclohexene in $30 \mathrm{~mL}$ of acetonitrile was placed in a Pyrex vessel $(\lambda>290 \mathrm{~nm})$, purged with nitrogen and irradiated for 6 hours using a medium pressure mercury lamp $(125 \mathrm{~W})$. The solvent was removed, and the residue was purified by flash chromatography (silica gel, $93 \%$ dichloromethane/ethyl acetate) to give two products as white solids.

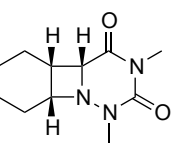

cis $\mathbf{D C H}<>\mathbf{A Z T}_{\mathrm{m}}(0.32 \mathrm{~g}, 41 \%):{ }^{1} \mathbf{H} \mathbf{N M R}\left(300 \mathrm{MHz}, \mathrm{CDCl}_{3}\right) \delta 4.33$ $(\mathrm{d}, \mathrm{J}=9.0 \mathrm{~Hz}, 1 \mathrm{H}), 4.01($ apparent q, $J=8.5 \mathrm{~Hz}, 1 \mathrm{H}), 3.15(\mathrm{~s}, 3 \mathrm{H}), 2.98$ cis DCH $>$ AZT ${ }_{\mathrm{m}} \quad(\mathrm{s}, 3 \mathrm{H}), 2.93(\mathrm{~m}, 1 \mathrm{H}), 2.04-1.93(\mathrm{~m}, 1 \mathrm{H}), 1.84-1.69(\mathrm{~m}, 1 \mathrm{H}), 1.65-$ $1.30(\mathrm{~m}, 4 \mathrm{H}), 0.99-0.87 \mathrm{ppm}(\mathrm{m}, 2 \mathrm{H}) .{ }^{13} \mathbf{C}$ NMR (75 MHz, $\left.\mathrm{CDCl}_{3}\right) \delta 170.0(\mathrm{C})$, $152.5(\mathrm{C}), 66.0(\mathrm{CH}), 63.9(\mathrm{CH}), 36.2(\mathrm{CH}), 30.4\left(\mathrm{CH}_{3}\right), 26.8\left(\mathrm{CH}_{3}\right), 23.3\left(\mathrm{CH}_{2}\right), 22.0$ $\left(\mathrm{CH}_{2}\right), 21.4\left(\mathrm{CH}_{2}\right), 19.9 \mathrm{ppm}\left(\mathrm{CH}_{2}\right)$. HRMS (ESI): $\mathrm{m} /$ \% calcd for $\mathrm{C}_{11} \mathrm{H}_{18} \mathrm{~N}_{3} \mathrm{O}_{2}$ $[\mathrm{M}+\mathrm{H}]^{+}$224.1399, found 224.1407.

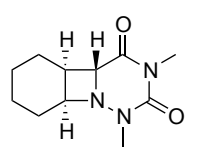

trans $\mathbf{D C H}<>\mathbf{A Z T}_{\mathrm{m}}(0.28 \mathrm{~g}, 36 \%):{ }^{1} \mathbf{H} \mathbf{N M R}\left(300 \mathrm{MHz}, \mathrm{CDCl}_{3}\right) \delta$ $4.03-3.91(\mathrm{~m}, 2 \mathrm{H}), 3.24(\mathrm{~s}, 3 \mathrm{H}), 3.19(\mathrm{~s}, 3 \mathrm{H}), 2.42(\mathrm{q}, J=8.8 \mathrm{~Hz}, 1 \mathrm{H})$, $2.20-1.58(\mathrm{~m}, 5 \mathrm{H}), 1.49-1.31(\mathrm{~m}, 1 \mathrm{H}), 1.16-0.96 \mathrm{ppm}(\mathrm{m}, 1 \mathrm{H}) .{ }^{13} \mathrm{C}$ NMR $\left(75 \mathrm{MHz}, \mathrm{CDCl}_{3}\right) \delta 171.4(\mathrm{C}), 151.9(\mathrm{C}), 67.1(\mathrm{CH}), 66.9(\mathrm{CH})$, $38.4(\mathrm{CH}), 33.0\left(\mathrm{CH}_{3}\right), 28.1\left(\mathrm{CH}_{2}\right), 27.4\left(\mathrm{CH}_{2}\right), 27.1\left(\mathrm{CH}_{3}\right), 22.2\left(\mathrm{CH}_{2}\right), 20.2\left(\mathrm{CH}_{2}\right)$. HRMS (ESI): $m / z$ calcd for $\mathrm{C}_{11} \mathrm{H}_{18} \mathrm{~N}_{3} \mathrm{O}_{2}[\mathrm{M}+\mathrm{H}]^{+} 224.1399$, found 224.1392 .

\subsubsection{NMR Spectra $\left({ }^{1} \mathrm{H}, \mathrm{DEPT},{ }^{13} \mathrm{C}\right)$}


Chapter 3: Photoreduction of an Azetidine Model of the (6-4) Photoproduct

$$
\text { 至 }
$$
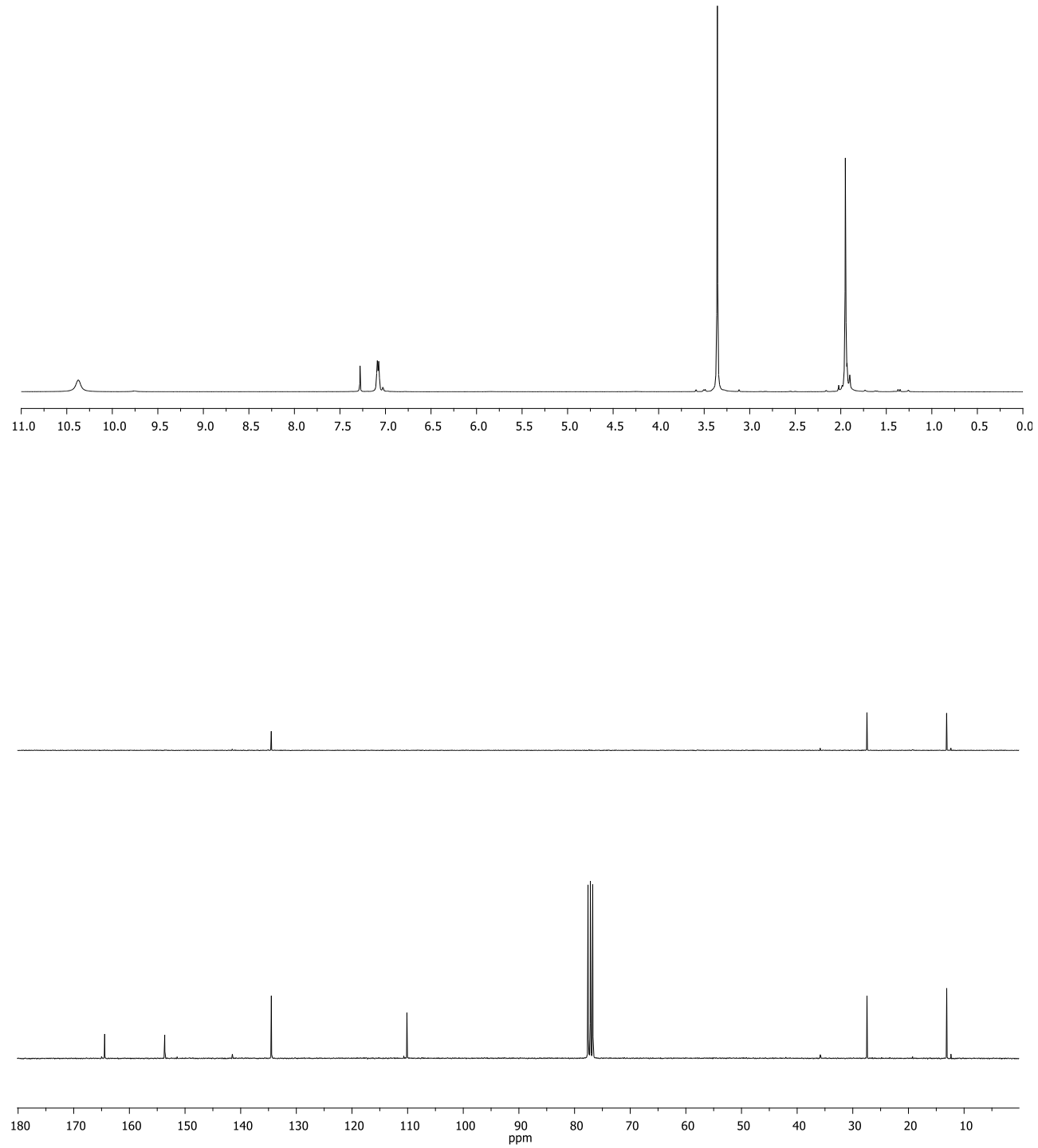

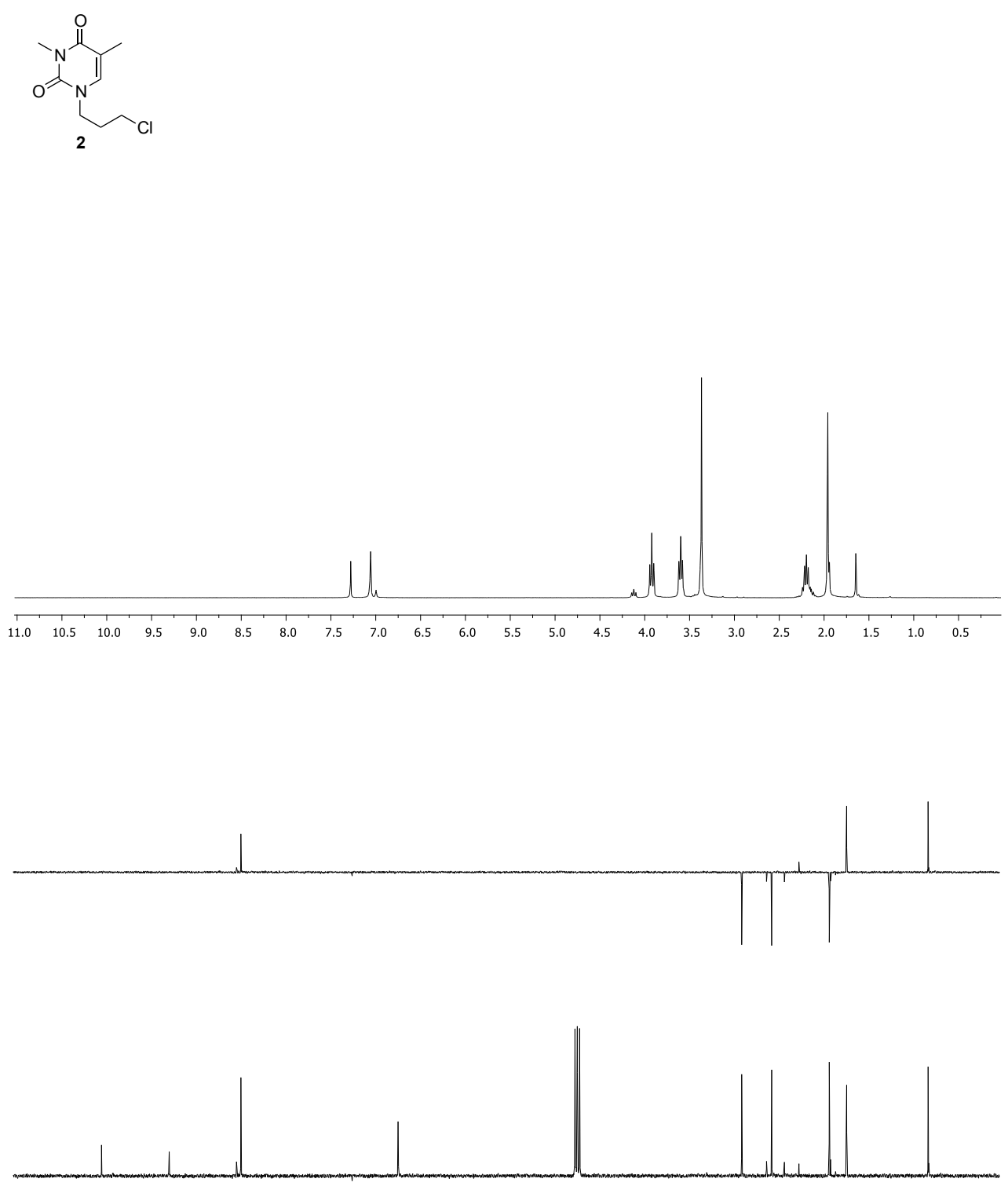

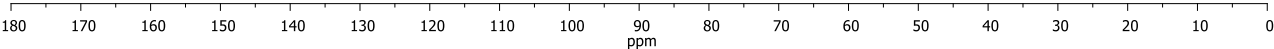


Chapter 3: Photoreduction of an Azetidine Model of the (6-4) Photoproduct
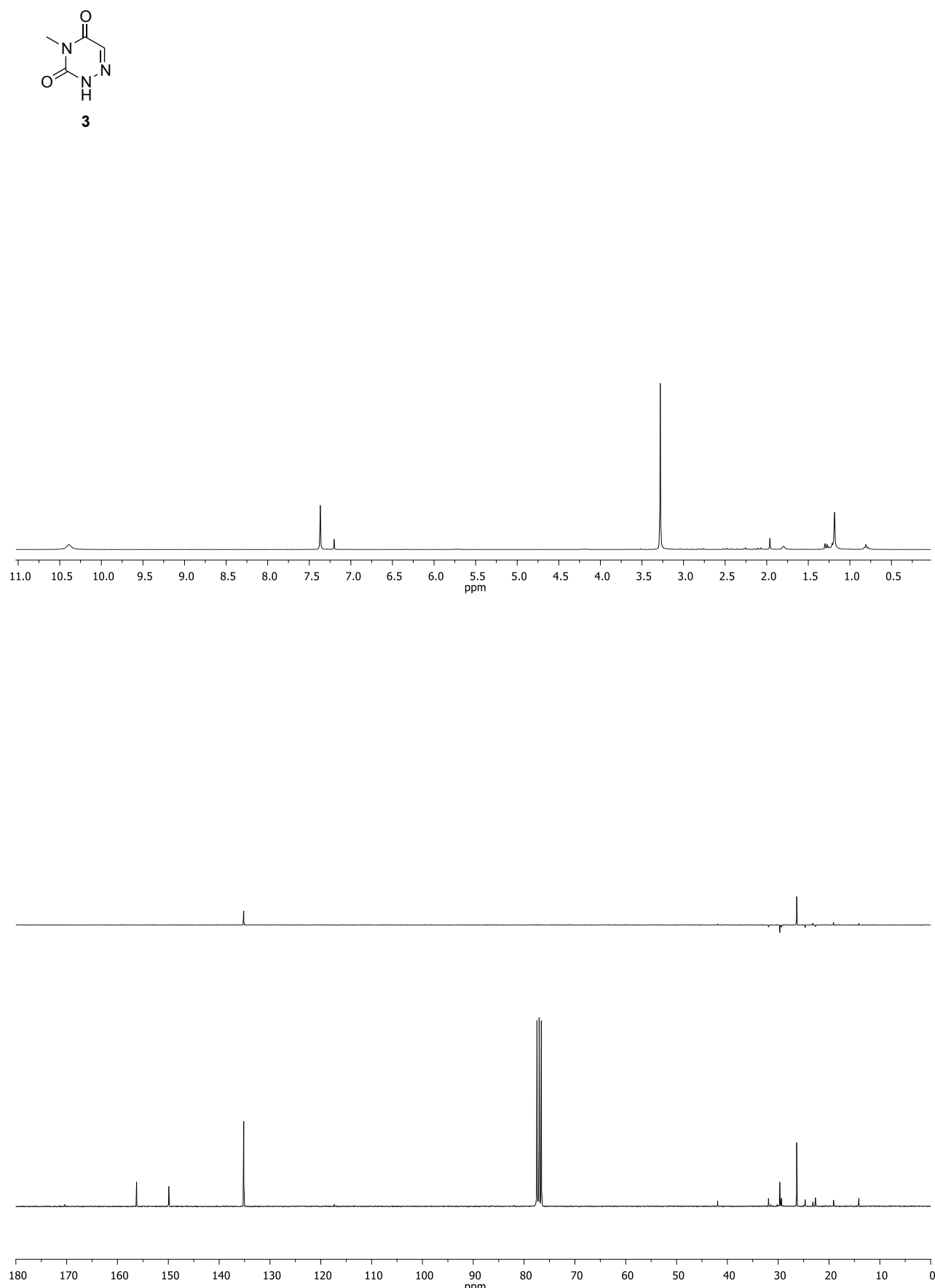
Chapter 3: Photoreduction of an Azetidine Model of the (6-4) Photoproduct
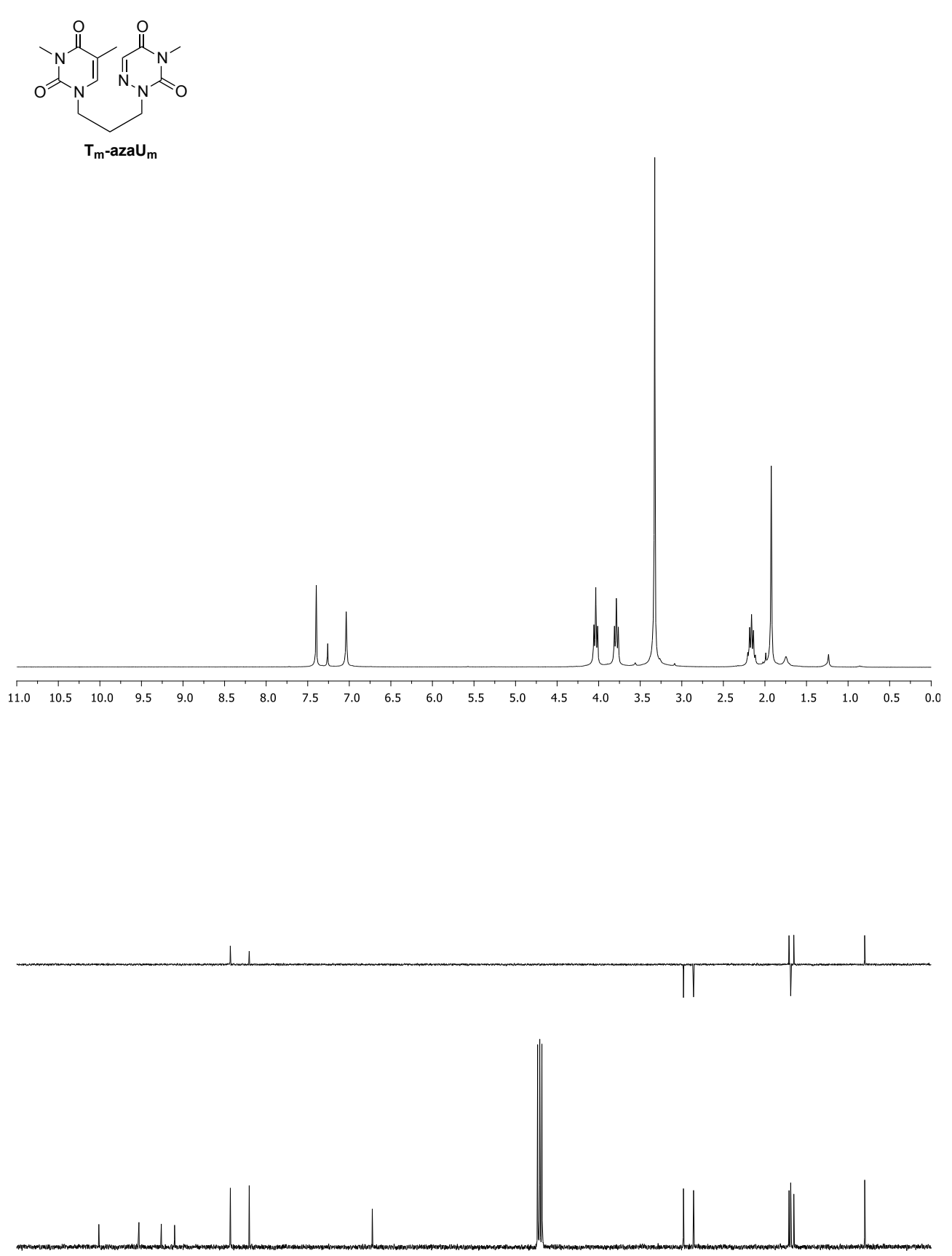

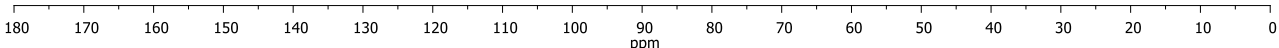



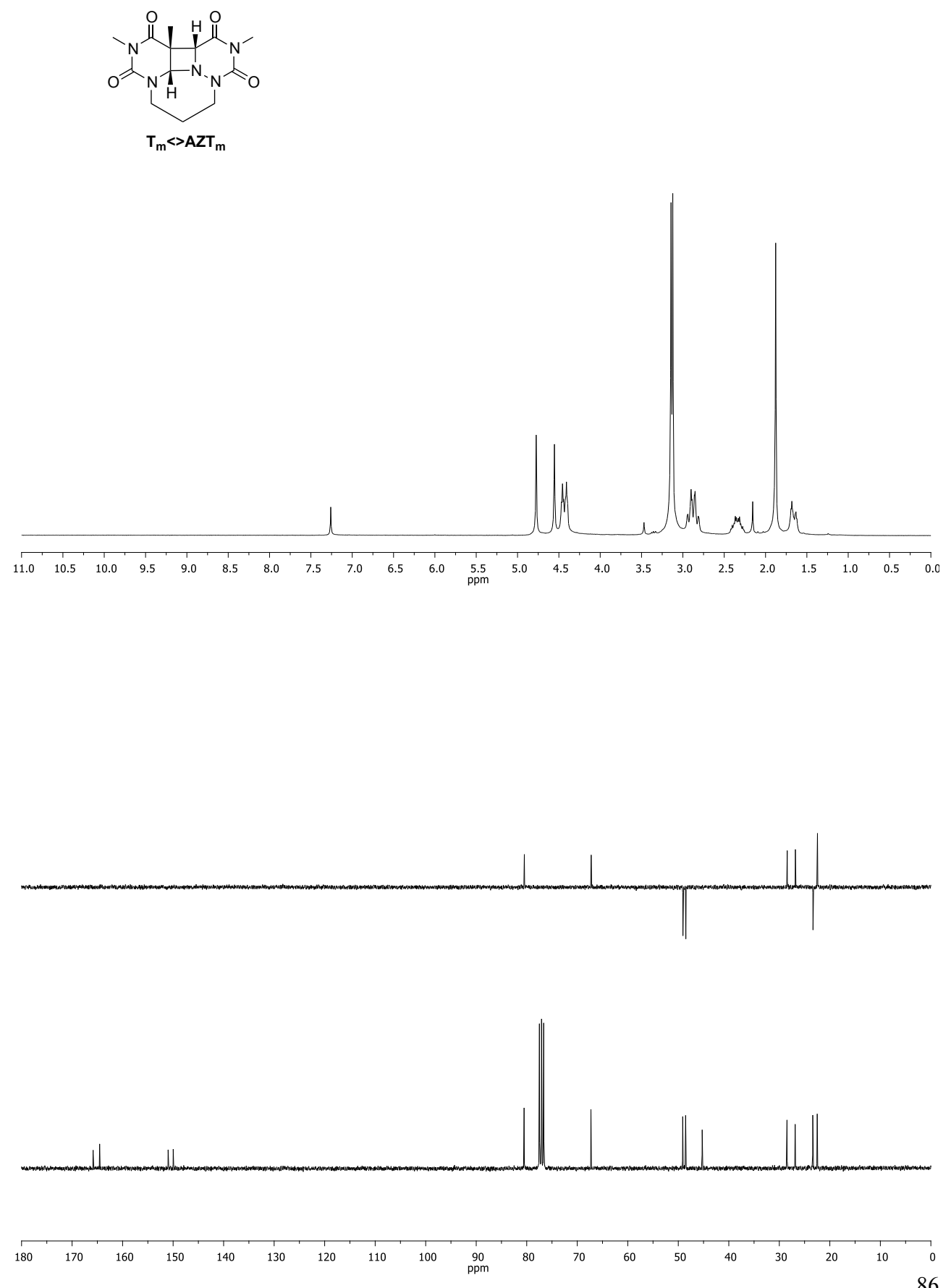


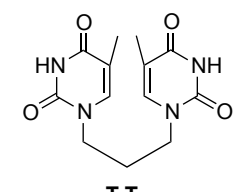

T-T
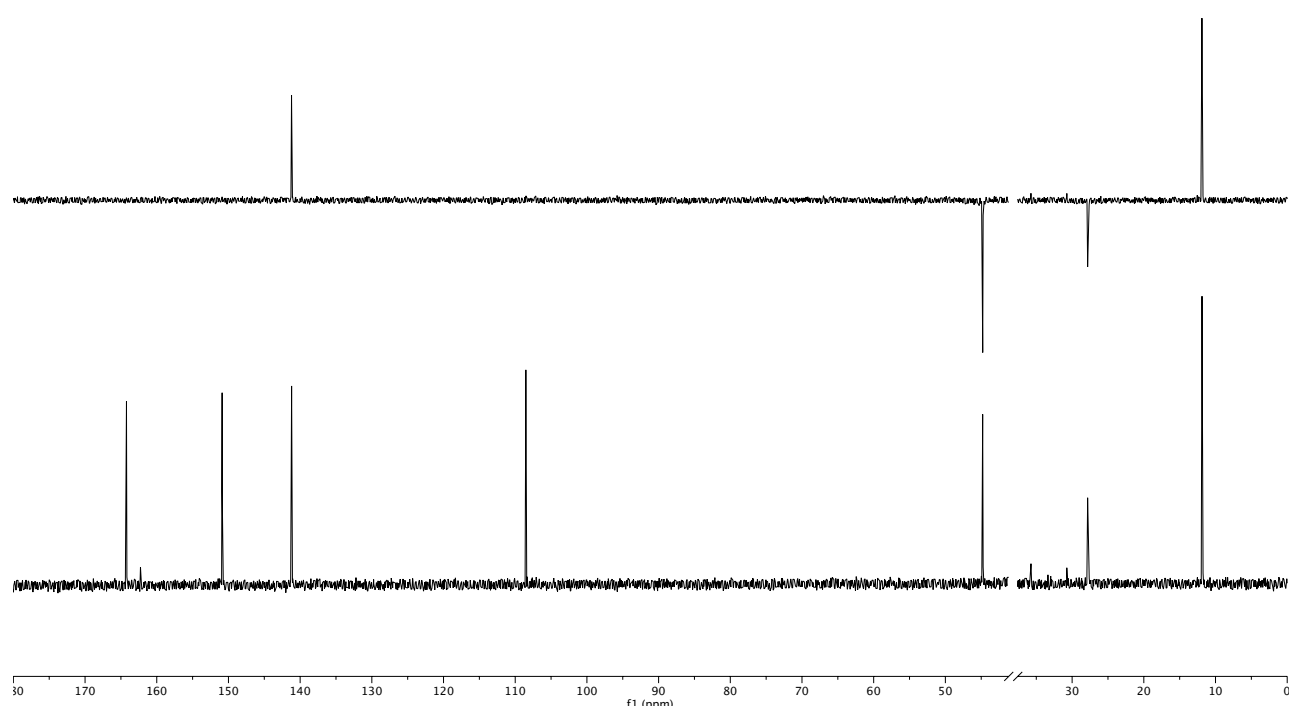


$$
\text { (c) }
$$
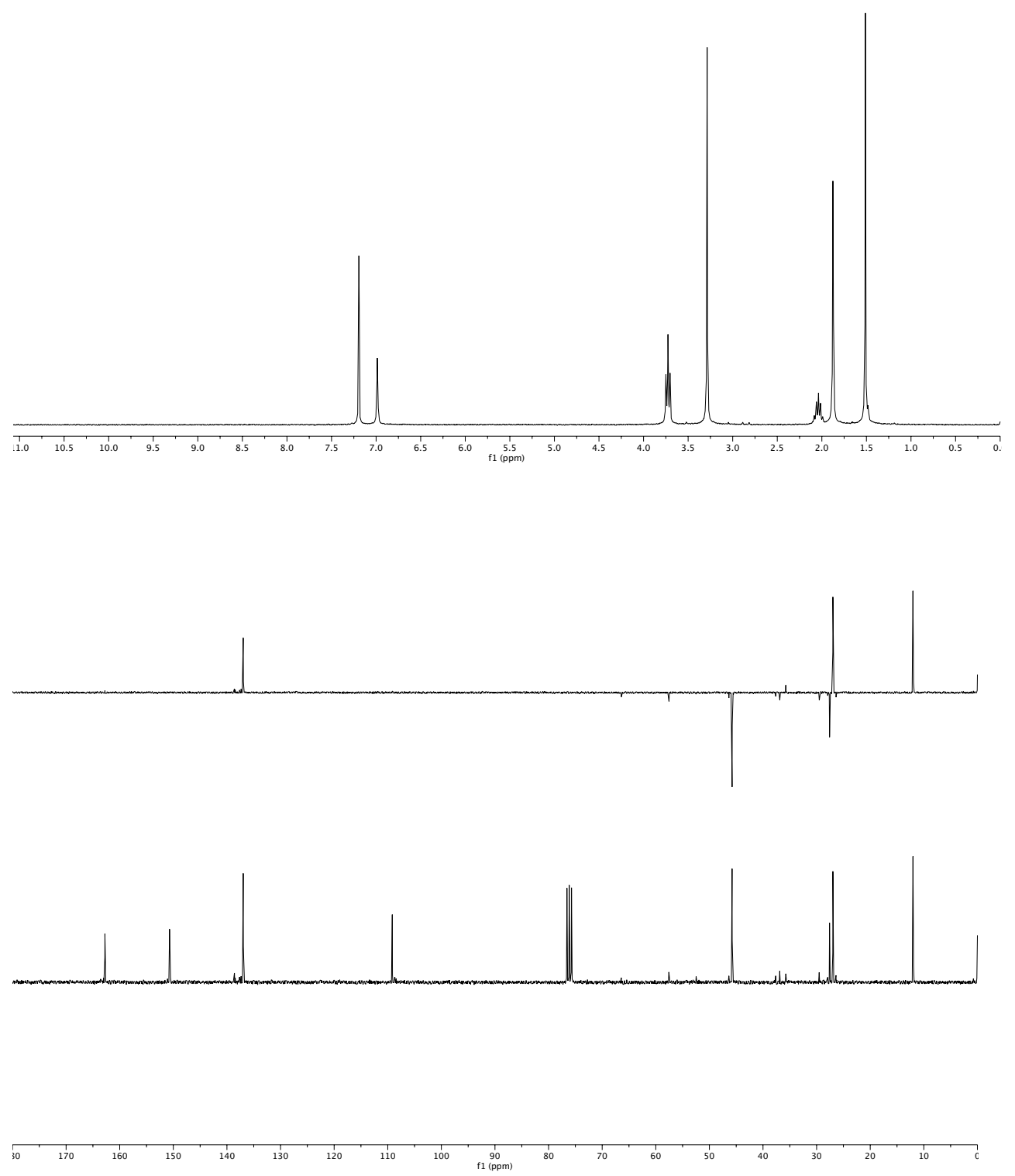

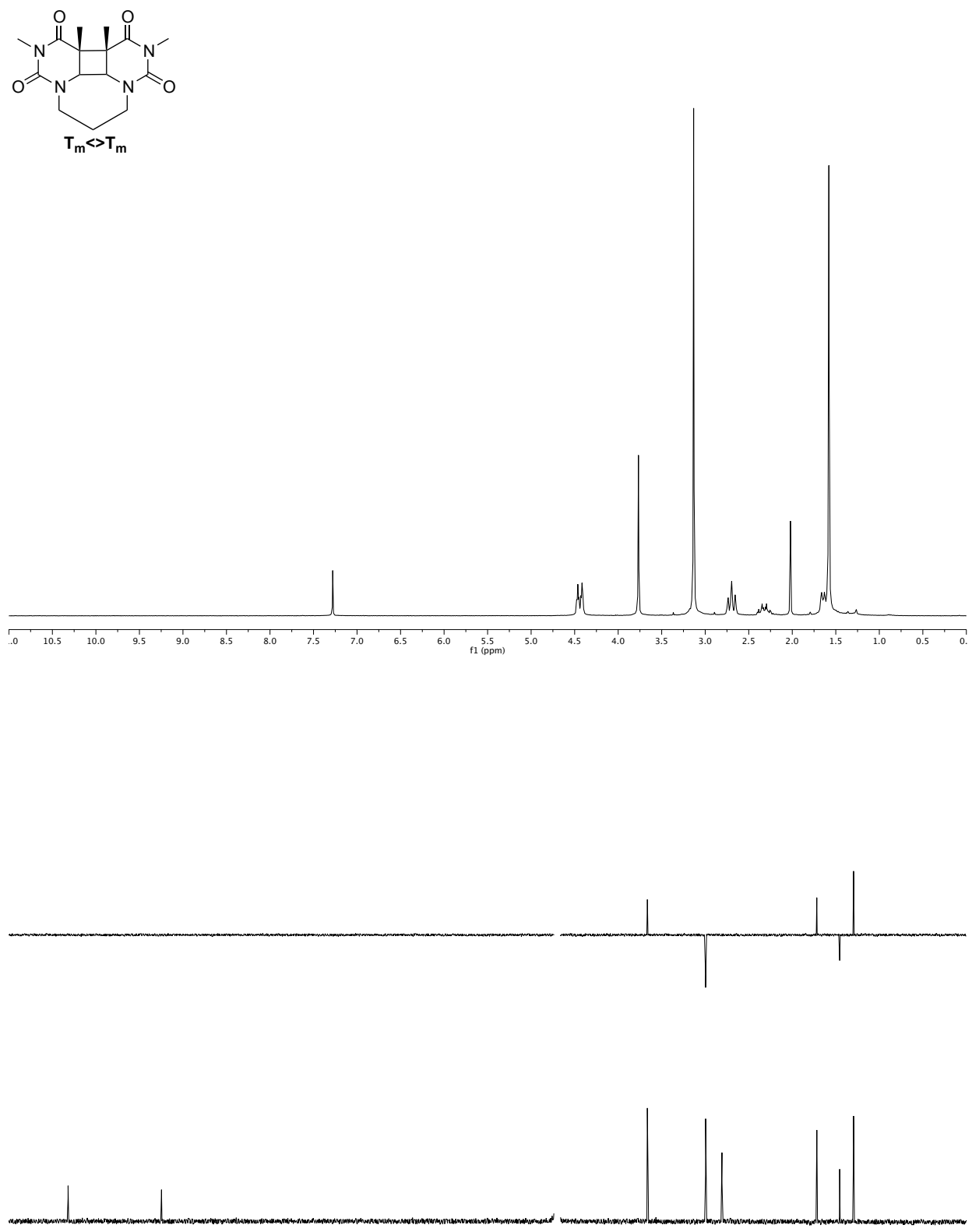
Chapter 3: Photoreduction of an Azetidine Model of the (6-4) Photoproduct
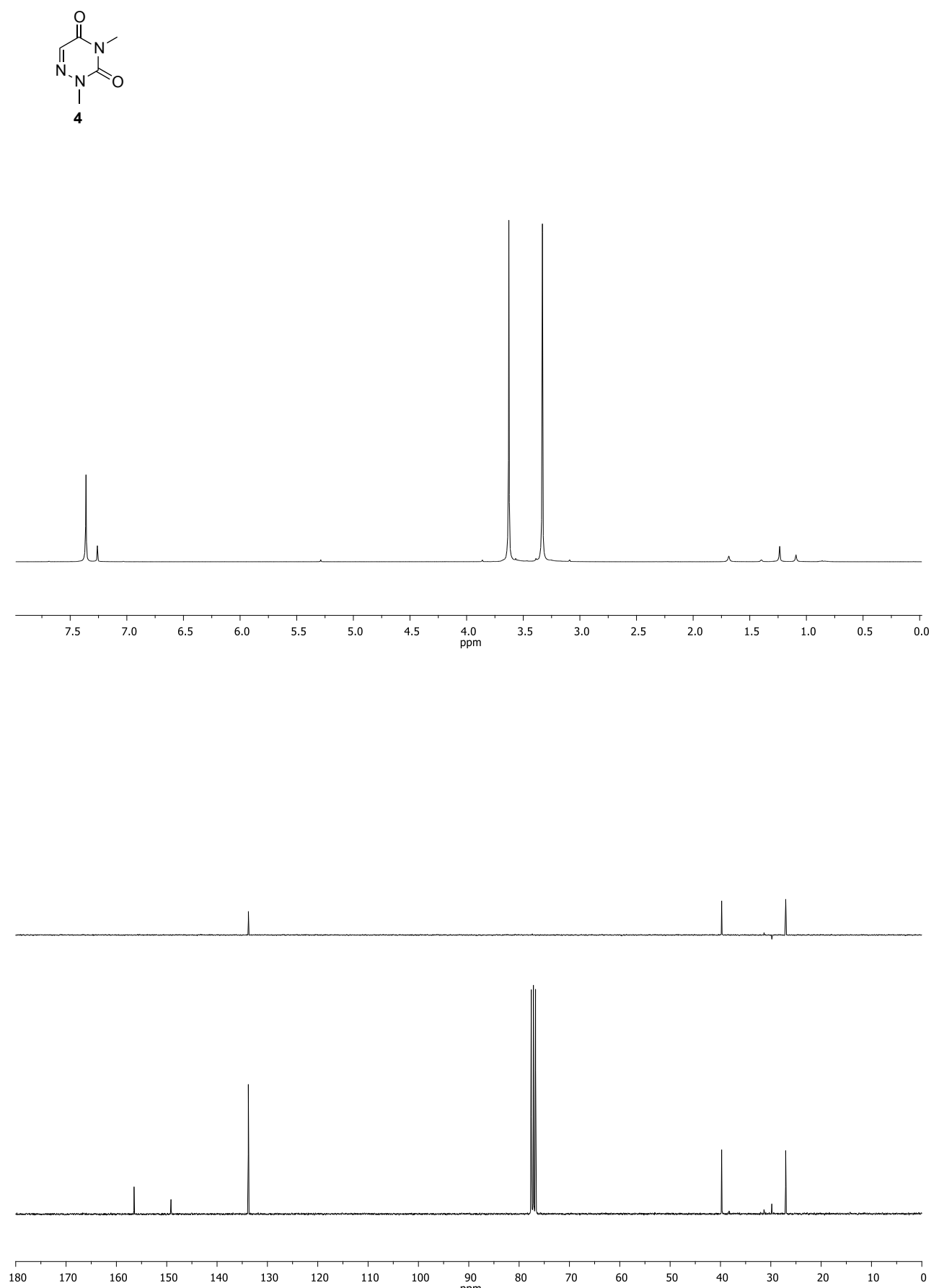


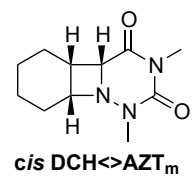

cis $\mathrm{DCH}<>\mathrm{AZT}_{\mathrm{m}}$
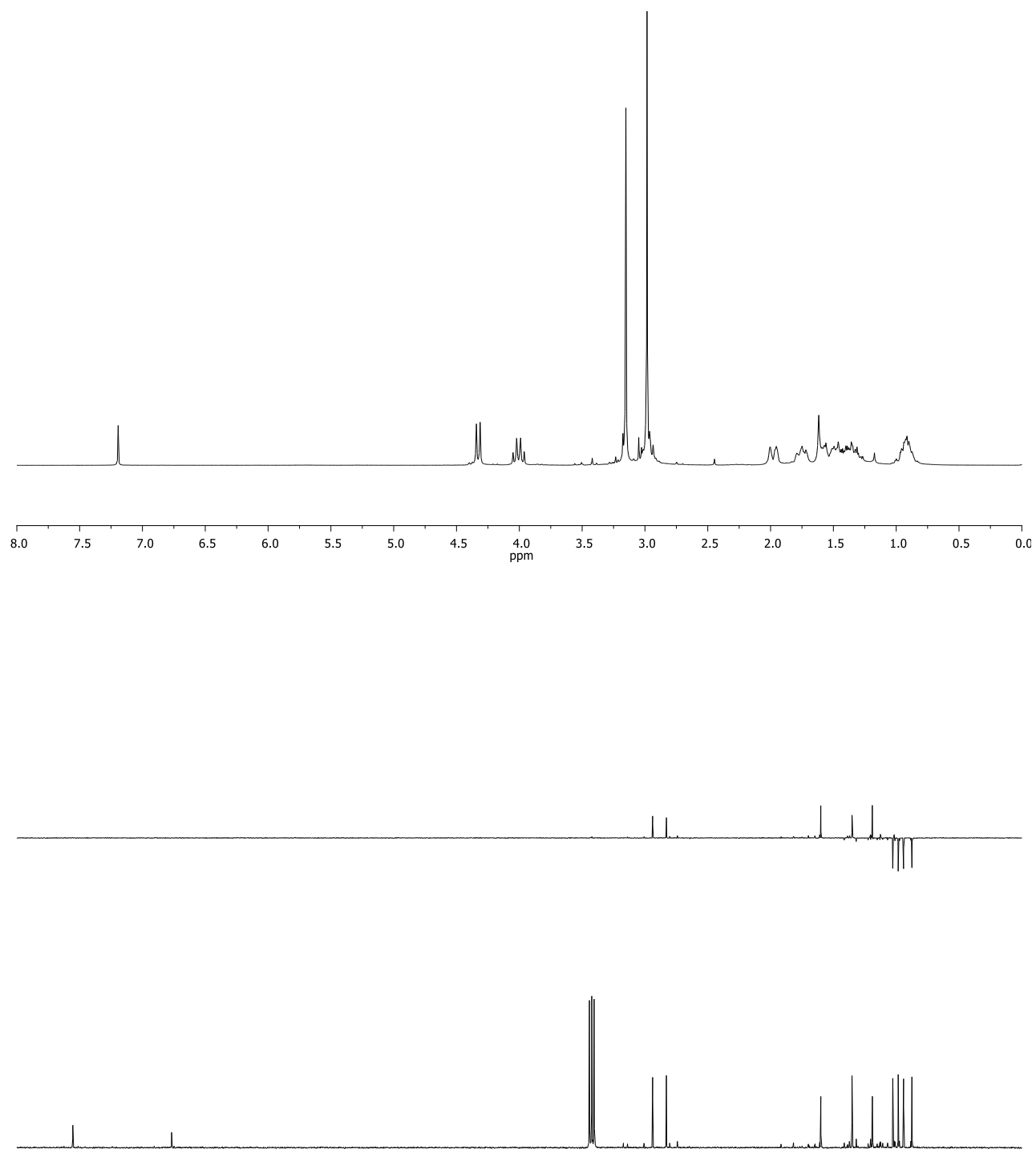

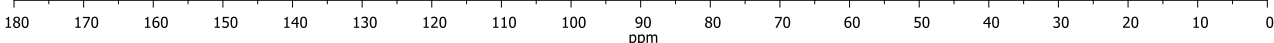


Chapter 3: Photoreduction of an Azetidine Model of the (6-4) Photoproduct
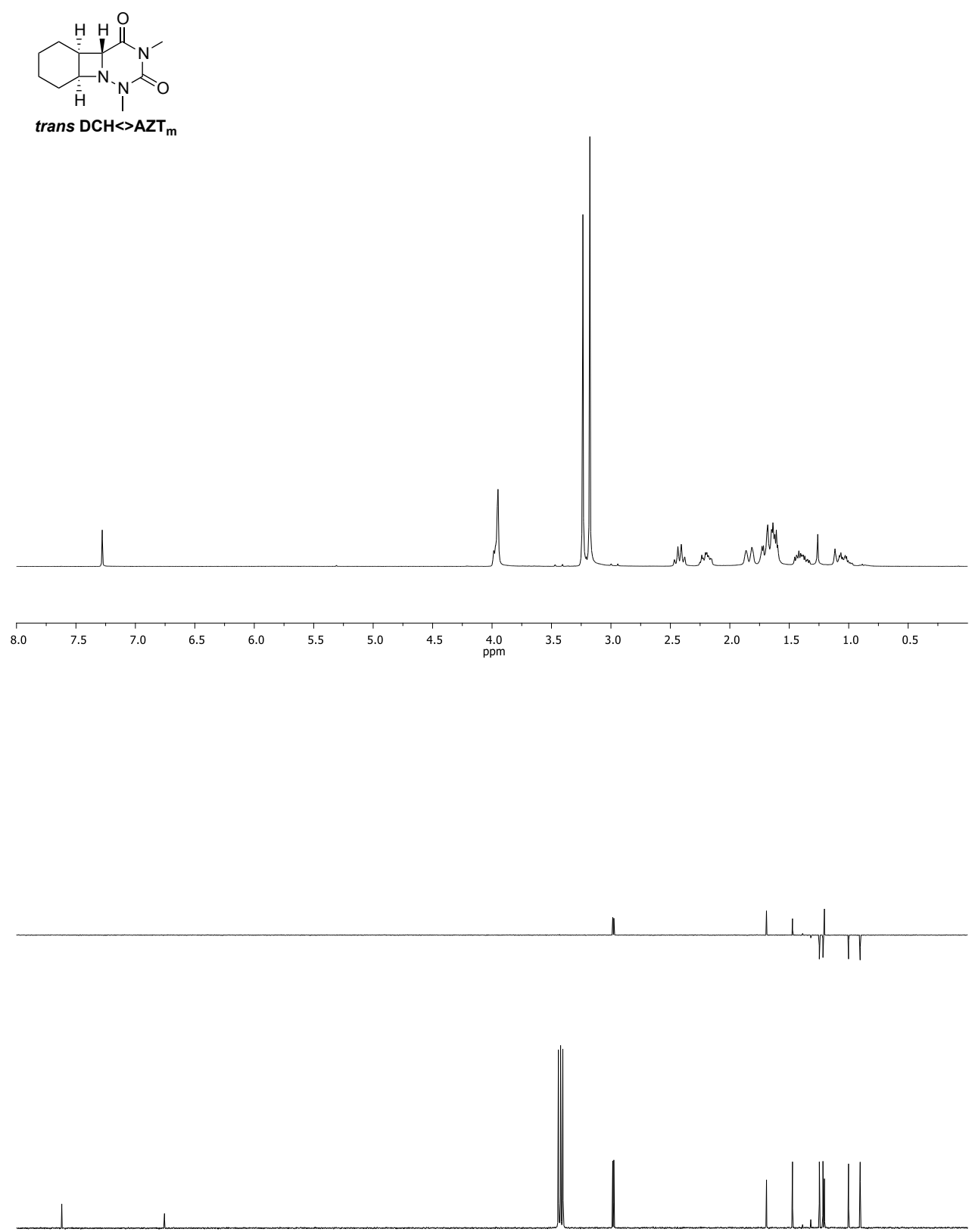

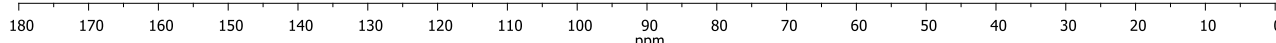




\subsubsection{Annex}

Figure S1. NOESY spectrum of compounds cis and trans $\mathbf{D C H}<>\mathbf{A Z T}_{\mathrm{m}}$.
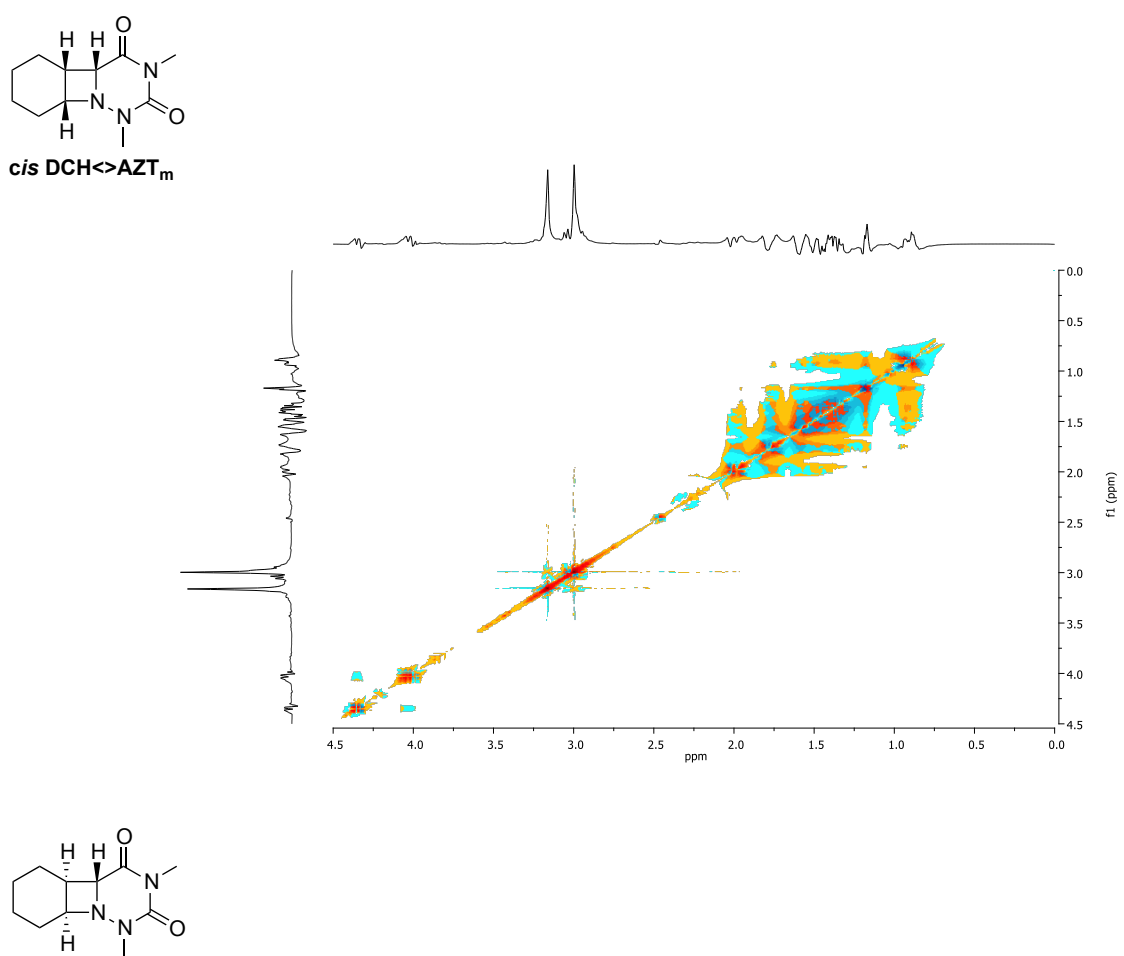

trans $\mathrm{DCH}<>\mathrm{AZT}_{\mathrm{m}}$

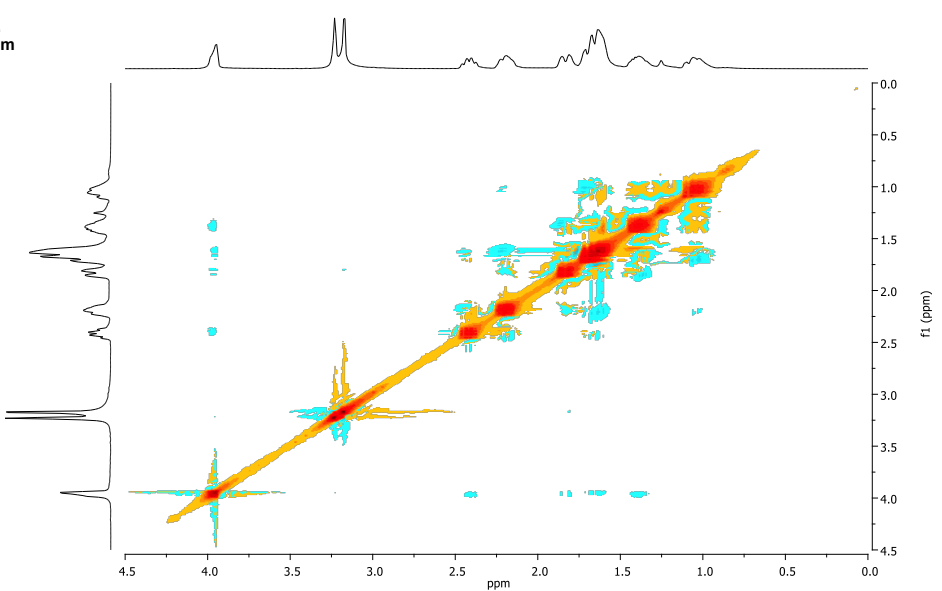




\subsection{References}

1. Gustafsson,

C.

M., http://www.nobelprize.org/nobel_prizes/chemistry/laureates/2015/advanced.html.

2. Yamamoto, J.; Plaza, P.; Brettel, K., Repair of (6-4) lesions in DNA by (6-4) photolyase: 20 years of quest for the photoreaction mechanism. Photochem. Photobiol. 2017, 93, 51-66.

3. Liu, Z.; Tan, C.; Guo, X.; Kao, Y. T.; Li, J.; Wang, L.; Sancar, A.; Zhong, D., Dynamics and mechanism of cyclobutane pyrimidine dimer repair by DNA photolyase. Proc. Natl. Acad. Sci. 2011, 108, 14831-14836.

4. Li, J.; Liu, Z.; Tan, C.; Guo, X.; Wang, L.; Sancar, A.; Zhong, D., Dynamics and mechanism of repair of ultraviolet-induced (6-4) photoproduct by photolyase. Nature 2010, 466, 887-890.

5. Perez-Ruiz, R.; Jimenez, M. C.; Miranda, M. A., Hetero-cycloreversions mediated by photoinduced electron transfer. Acc. Chem. Res. 2014, 47, 1359-1368.

6. Padwa, P., Photochemistry of the carbon-nitrogen double bond. Chem. Rev. 1977, 77, 37-68.

7. Cuquerella, M. C.; Lhiaubet-Vallet, V.; Bosca, F.; Miranda, M. A., Photosensitised pyrimidine dimerisation in DNA. Chem. Sci. 2011, 2, 1219-1232.

8. Kawamura, Y.; Kumagai, T.; Mukai, T., Photocycloaddition reaction of 3-aryl2-isoxazolines with indene. Chem. Lett. 1985, 14, 1937-1940.

9. Sampedro, D., Computational exploration of the photocycloaddition of imines to alkenes. ChemPhysChem 2006, 7, 2456-2459.

10. Oe, K.; Tashiro, M.; Tsuge, O., Photochemistry of heterocyclic compounds. Photochemical reaction of 2,5-diaryl-1,3,4-oxadiazoles with indene. J. Org. Chem. 1977, 42, 1496-1499.

11. Anderson, D. R.; Keute, J. S.; Koch, T. H.; Moseley, R. H., Di-tert-butyl nitroxide quenching of the photoaddition of olefine to the carbon-nitrogen double bond of 3-ethoxyisoindolenone. J. Am. Chem. Soc. 1977, 99, 6332-6340. 
12. Swenton, J. S.; Hyatt, J. A., Photosensitized cycloadditions to 1,3-dimethyl-6azauracil and 1,3-dimethyl-6-azathymine. An imine linkage unusually reactive toward photocycloaddition. J. Am. Chem. Soc. 1974, 96, 4879-4885.

13. Kobayashi, T.; Harada, Y.; Suzuki, T.; Ichimura, T., Excited state characteristics of 6-azauracil in acetonitrile: drastically different relaxation mechanism from uracil. J. Phys. Chem. A 2008, 112, 13308-13315.

14. Perez-Ruiz, R.; Saez, J. A.; Domingo, L. R.; Jiménez, M. C.; Miranda, M. A., Ring splitting of azetidin-2-ones via radical anions. Org. Biomol. Chem. 2012, 10, 79287932.

15. Perez-Ruiz, R. S., J. A.; Jimenez, M. C.; Miranda, M. A., Cycloreversion of beta-lactams via photoinduced electron transfer. Org. Biomol. Chem. 2014, 12, 84288432.

16. Zasada-Parzynska, A.; Celewicz, L.; Golankiewicz, K., Synthesis and photochemical properties of pyrimidine-6-azapyrimidine analogs of dinucleotides with propanone bridge. Synth. Commun. 1984, 16, 1177-1185.

17. Jankowska, J.; Koroniak, H.; Golankiewicz, K., The influence of alkyl substituents on internal photodimerization of pyrimidine-6-azapyrimidine dinucleotide analogues. Heterocycles 1984, 22, 1363-1368.

18. Golankiewicz, K.; Jankowska, J.; Koroniak, H., Internal photodimerization of some pyrimidine-6-azapyrimidine dinucleotide analogues. Heterocycles 1984, 22, 67-72.

19. Kochevar, I. E.; Wagner, P. J., Quenching of triplet phenyl ketones by olefins. J. Am. Chem. Soc. 1972, 94, 3859-3865.

20. Tatake, V. G.; Desai, T. S.; Sane, P. V., Isothermal luminescence and thermoluminescence of nucleic acid bases following gamma irradiation. Photochem. Photobiol. 1976, 24, 463-472.

21. Leonard, N.; Golankiewicz, K.; McCredie, R.; Johnson, S.; Paul, I., Synthetic spectroscopic models related to coenzymes and base pairs. III. A 1,1'-trimethylenelinked thymine photodimer of cis-syn structure. J. Am. Chem. Soc. 1969, 91, 5855-5862. 
Chapter 3: Photoreduction of an Azetidine Model of the (6-4) Photoproduct

22. Leonard, N. J., Trimethylene bridges as synthetic spacers for the detection of intramolecular interactions. Acc. Chem. Res. 1979, 12, 423-429.

23. Browne, D. T. E., J.; Leonard, N. J., Synthetic spectroscopic models related to coenzymes and base pairs. II Evidence for intramolecular base-base interactions in dinucleotide analogs. J. Am. Chem. Soc. 1968, 90, 7302-7323.

24. Yeh, S. R.; Falvey, D. E., Model studies of DNA photorepair: energetic requirements for the radical anion mechanism determined by fluorescence quenching. J. Am. Chem. Soc. 1992, 114, 1971-1977.

25. Scannell, M. P.; Fenick, D. J.; Yeh, S.; Falvey, D. E., Model studies of DNA photorepair: reduction potentials of thymine and cytosine cyclobutane dimers measured by fluorescence quenching. J. Am. Chem. Soc. 1997, 119, 1971-1977.

26. Hartman, R. F.; Rose, S. D.; Pouwels, P. J. W.; Kaptein, R., Flavin-sensitized photochemically induced dynamid nuclear polarization detection of pyrimidine dimer radicals. Photochem. Photobiol. 1992, 56, 305-310.

27. Pouwels, J. W.; Kaptein, R.; Hartman, R. F.; Rose, S. D., Photo-CIDNP study of pyrimidine dimer splitting I: reactions involving pyrimidine radical cation intermediates. Photochem. Photobiol. 1995, 61, 563-574.

28. Kao, Y. T.; Saxena, C.; He, T. F.; Guo, L.; Wang, L.; Sancar, A.; Zhong, D., Ultrafast dynamics of flavins in five redox states. J. Am. Chem. Soc. 2008, 130, 1313213139.

29. Kao, Y. T. S., Q. H.; Saxena, C.; Wang, L.; Zhong, D., Dynamics and mechanism of DNA repair in a biomimetic system: flavin-thymine dimer adduct. $J$. Am. Chem. Soc. 2012, 134, 1501-1503.

30. Trzcionka, J.; Lhiaubet-Vallet, V.; Paris, C.; Belmadoui, N.; Climent, M. J.; Miranda, M. A., Model studies on a carprofen derivative as dual photosensitizer for thymine dimerization and (6-4) photoproduct repair. ChemBioChem 2007, 8, 402-407. 
31. Boussicault, F.; Kruger, O.; Robert, M.; Wille, U., Dissociative electron transfer to and from pyrimidine cyclobutane dimers: An electrochemical study. Org. Biomol. Chem. 2004, 2, 2742-2750.

32. Boussicault, F. R., M., Electron transfer in DNA and in DNA-related biological processes. Electrochemical insights. Chem. Rev. 2008, 108, 2622-2645.

33. Ide, H.; Otsuki, N.; Nishimoto, S.; Kagiya, T., Photoreduction of thymine glycol sensitized by aromatics amines in aqueous solution. J. Chem. Soc. Perkin. Trans. 2 $1985,1387-1392$.

34. Durbeej, B.; Eriksson, L. A., Thermodynamics of the photoenzymatic repair mechanism studied by density functional theory. J. Am. Chem. Soc. 2000, 122, 1012610132. 

Chapter 4:

Study of Azetidines Cycloreversion by Oxidative Electron Transfer 



\subsection{Introduction}

As mentioned previously, the UV-induced CPDs and 6-4PPs are repaired by photolyases through a photoreductive process from the reduced flavin cofactor $\mathrm{FADH}^{-}$. However, although CPD photolyase uses a reductive single electron transfer to initiate the cycloreversion, it has been shown that the cycloreversion of the CPD can also be achieved in vitro through an oxidative pathway. ${ }^{1}$ In this context, an important result has been the long range repair of thymine dimers incorporated in DNA duplex by means of a reaction involving an electron transfer to a rhodium intercalator activated with visible light. ${ }^{2}$ This result opened the door to consider the photooxidative approach as a relevant tool for DNA therapies, and thus, to develop new photoactive chemotherapeutic agents.

In model systems, numerous molecules and ions have been found to be able to split photochemically $\mathrm{T}<>\mathrm{T}$ in solution through an oxidative pathway. ${ }^{3-7}$ Most of the experimental investigations were performed using photosensitizers to oxidize $\mathrm{T}<>\mathrm{T}$, but oxidizing radicals and radical ions were also employed. Compounds such as potassium hexacyanoferrate (III) $\left(\mathrm{K}_{3} \mathrm{Fe}(\mathrm{CN})_{6}{ }^{7}\right.$ and uranyl(IV) sulfate $\left(\mathrm{UO}_{2} \mathrm{SO}_{4}\right)^{7}$ have been reported as inorganic photooxidants, or anthraquinone-2-sulfonate (AQS) ${ }^{4}$, anthraquinone $(\mathrm{AQ})^{5}$, 9,10-dicyanoanthracene (DCA)4, 2,3-dichloro-5,6-dicyano-1,4-quinone (DDQ) $)^{5}$ and protonated 2', 3', 4', 5'-tetraacetylriboflavin $\left(\mathrm{Ac}_{4} \mathrm{rfH}^{+}\right)^{6,8}$ for the organic counterparts (Figure 4.1).
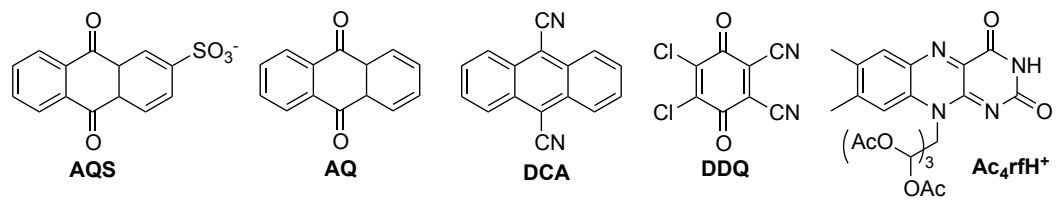

Figure 4.1. Most important photooxidants used in the studies for oxidative repair of $\mathrm{T}<>\mathrm{T}$ dimers. 
The assumed mechanism for oxidative splitting of $\mathrm{T}<>\mathrm{T}$ is given in Scheme 4.1. ${ }^{9}$ Electron transfer from $\mathrm{T}<>\mathrm{T}$ to the oxidant leads to formation of an unstable radical cation $\mathrm{T}^{\bullet+}<>\mathrm{T}$ of the dimer, with the charge located on the nitrogen atom, which rapidly fragments (lifetime $<1 \mathrm{~ns})^{10}$. However, as the radical cation is delocalized, it is not unreasonable to propose that the C5-C5' and C6-C6' bonds are equally weakened and thus that the cleavage of two cyclobutyl bonds might be a two-step, or a concerted process. The measurement of the isotope effects on the fragmentation of $\mathrm{C} 5-\mathrm{C} 5$ ' and C6-C6' bonds of a model photodimer by an anthraquinone-sensitized cleavage supported a stepwise mechanism where the first C6-C6' bond cleavage is effectively irreversible. ${ }^{11}$ Theoretical studies also supported the two-step mechanism via biradical structure formed after the opening C6-C6' bond. ${ }^{12}$ Therefore, the oxidative mechanism follows two-sequential cleavage of the C6-C6' bond and the C5-C5' bond, which finally leads to a repaired base and its radical cation $\mathrm{T}^{\bullet+}$. The latter was found to initiate a radical chain through oxidation of further dimer $\mathrm{T}<>\mathrm{T} .{ }^{13}$ Photochemically induced dynamic nuclear polarization (CIDNP) experiments using AQS as PS, demonstrated the existence of both $\mathrm{T}^{\bullet+}<>\mathrm{T}$ and its dissociation product, the monomer radical cation $\mathrm{T}^{\bullet+} \cdot{ }^{\cdot 14}$

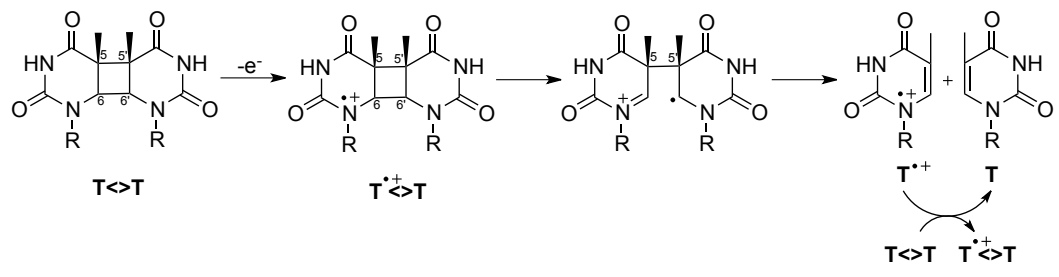

Scheme 4.1. Oxidative pathway for $\mathrm{T}<>\mathrm{T}$ splitting.

In case of 6-4PPs, theoretical calculations have studied the possible involvement of both the anionic and cationic pathways in the regeneration of native pyrimidines from azetidine and oxetane lesions. ${ }^{15}$ In this context, the oxidative cycloreversion has been widely reported for oxetane models with photooxidants such as cyanonaphtha- 
Chapter 4: Study of Azetidines Cycloreversion by Oxidative Electron Transfer

lenes or tetraacetylriboflavin; and involvement of the singlet excited state of the PS was supported by fluorescence experiments. ${ }^{16-17}$

A nonconcerted two-step mechanism has been proposed for the splitting on the basis of the loss of products stereochemistry. ${ }^{16}$ In addition, theoretical calculations supported this two-step mechanism for the 6-4PPs oxetane intermediate through an initial C-C bond breaking, followed by cleavage of the C-O bond. ${ }^{15}$ Interestingly, the detection of 1,4-radical cations involved in the cycloreversion of hydroxymethyldiphenyloxetane by intramolecular nucleophilic trapping with (thia)pyrylium salts showed that the process occurs through a nonconcerted mechanism starting with an initial cleavage of the $\mathrm{C}-\mathrm{O}$ bond. ${ }^{18}$

Although there are a plenty of research articles with regard to oxidative cycloreversion of oxetane models, only one work dealing with azetidine models has been reported until now. ${ }^{19}$ It addresses the cycloreversion of 1,2,3-triphenylazetidine achieved by oxidation with tris(4-bromophenyl)aminium hexachloroantimonate (also named BAHA) to lead to cis- and trans-stilbene, together with $N$-benzylideneaniline. The process was shown to ocurr through a stepwise cycloreversion of the azetidine radical through pathways a or b (Scheme 4.2).

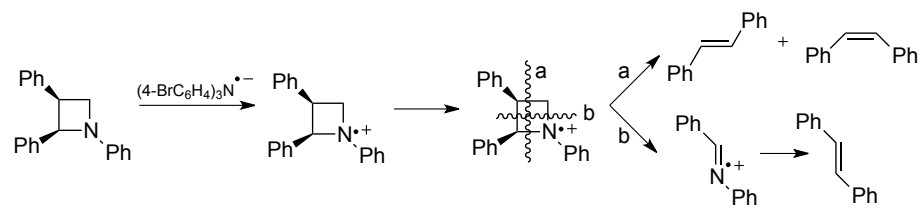

Scheme 4.2. Mechanistic pathways for the cycloreversion of azetidine radical cations.

In this context, oxidative electron transfer has only been investigated with triphenylazetidines, which are structurally unrelated to pyrimidine bases. Thus, in this chapter a photooxidative approach has been applied to the ring splitting of azabipyrimidinic azetidine $\mathbf{T}_{\mathrm{m}}<>\mathbf{A Z} \mathbf{T}_{\mathrm{m}}$ previously studied in Chapter 3. Moreover, 
parallel studies of photoinduced oxidative cycloreversion of $\mathbf{T}_{\mathrm{m}}<>\mathbf{T}_{\mathrm{m}}$ and $\mathbf{D C H}<>\mathbf{A Z T}_{\mathbf{m}}$ (Figure 4.2) have been included to investigate how the presence of the nitrogen atom in the four-membered ring affects in the ring splitting.

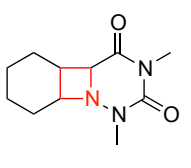

$\mathrm{DCH}<>\mathrm{AZT}_{\mathrm{m}}$

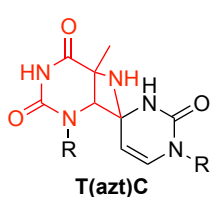

$\mathbf{T}($ azt)C
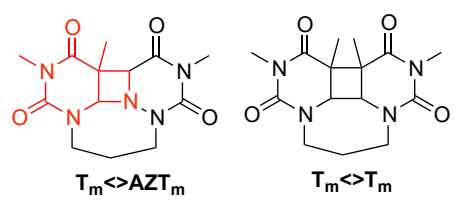

Figure 4.2. Structures of the azetidine models $\left(\mathbf{D C H}<>\mathbf{A Z} \mathbf{T}_{\mathrm{m}}\right.$ and $\mathbf{T}_{\mathrm{m}}<>\mathbf{A Z} \mathbf{T}_{\mathrm{m}}$ ) studied in this chapter, in relation with the intermediate $\mathbf{T}(\mathbf{a z t}) \mathbf{C}$ involved in the photorepair mechanism of 6-4PPs at TC sequences, and the cyclobutane derivative $\mathbf{T}_{\mathrm{m}}<>\mathbf{T}_{\mathrm{m}}$.

\subsection{Results and discussion}

\subsubsection{Fluorescence experiments}

Assuming that the photooxidative cycloreversion of azetidine is a nonconcerted two-step mechanism, two pathways can be proposed on the basis of the location of the formed radical cation. Thus, the photosensitizer is an electron acceptor in its excited state that, accepts an electron to generate the radical cation centered on the nitrogen atom of the thymine or azaU moiety of $\mathbf{T}_{\mathrm{m}}<>\mathbf{A Z} \mathbf{T}_{\mathrm{m}}$, followed by a two-step cleavage of C6-C6' and C5-C5' to lead to the repaired bases $\mathbf{T}_{\mathbf{m}}$-azaU $\mathbf{U}_{\mathbf{m}}$ as shown in Scheme 4.3.

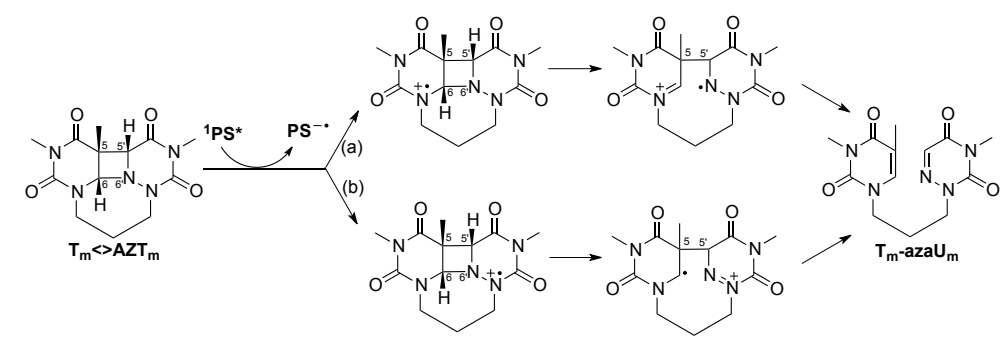

Scheme 4.3. Oxidative PET mechanisms of $\mathbf{T}_{\mathrm{m}}<>\mathrm{AZ} \mathbf{T}_{\mathrm{m}}$ in the presence of PS. 
In a first step, fluorescence experiments were performed with photooxidants in order to evaluate the possibility of an electron transfer process from $\mathbf{T}_{\mathrm{m}}<>\mathbf{A Z} \mathbf{T}_{\mathrm{m}}$ to the singlet-excited state of the photosensitizer. In addition, the same experiments were achieved for $\mathbf{T}_{\mathrm{m}}<>\mathbf{T}_{\mathrm{m}}$ and $\mathbf{D C H}<>\mathbf{A Z} \mathbf{T}_{\mathrm{m}}$ in order to know which moiety is key in the photooxidative process.

Therefore, 1,4-dicyanonaphthalene (DCN) and 9,10-dicyanoanthracene (DCA) were selected as the electron acceptors (Figure 4.3), their reduction potential in the singlet excited state $\left(\mathrm{E}_{\text {red }}^{\mathrm{D}^{*}}\right)$ being of ca. 2.4 and $1.8 \mathrm{~V}$ vs. $\mathrm{Ag} / \mathrm{AgCl}$, respectively.20

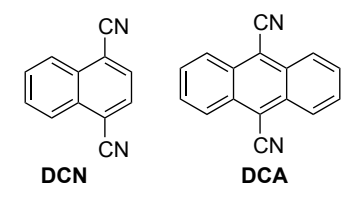

Figure 4.3. Structure of the used photosensitizers (PS).

Acetonitrile solutions of the photosensitizer were prepared with an absorbance of ca. 0.15 at the excitation wavelength $\left(\lambda_{\text {exc }}=310\right.$ or $\left.375 \mathrm{~nm}\right)$ and the fluorescence intensity was measured in the absence and in the presence of $\mathbf{T}_{\mathrm{m}}<>\mathbf{A Z T}_{\mathrm{m}}, \mathbf{T}_{\mathrm{m}}<>\mathbf{T}_{\mathrm{m}}$ or $\mathbf{D C H}<>\mathbf{A Z T}_{\mathrm{m}}$. As shown in Figure 4.4, steady-state fluorescence measurements revealed a strong decrease of the emission intensity of DCN and DCA for $\mathbf{T}_{\mathrm{m}}<>\mathbf{A Z T}_{\mathrm{m}}$ with $\mathrm{k}_{\mathrm{q}}$, obtained from Stern-Volmer plots, of 72.8 and $7.0 \times 10^{9} \mathrm{M}^{-1} \mathrm{~s}^{-1}$, respectively. By contrast, small quenching of the photosensitizer was obtained for $\mathbf{T}_{\mathrm{m}}<>\mathbf{T}_{\mathrm{m}}$ and lower values of $\mathrm{k}_{\mathrm{q}}$ of ca. 15.8 and $0.4 \times 10^{9} \mathrm{M}^{-1} \mathrm{~s}^{-1}$ were determined for the DCN and DCA (Figure 4.4), respectively.

However, the rate constant for DCN/ $\mathbf{T}_{\mathrm{m}}<>\mathbf{A Z} \mathbf{T}_{\mathrm{m}}$ was abnormally high as it is faster than the diffusion control in acetonitrile, $\mathrm{k}_{\text {diff }}$ of ca. $1.9 \times 10^{10} \mathrm{M}^{-1} \mathrm{~s}^{-1}$. Actually, partial light absorption by the concentrated quencher occurs when the sample is excited at $310 \mathrm{~nm}$, reducing the portion of light absorbed by the photosensitizer, which results in a decrease of the emission intensity. By contrast, the longer excitation wave- 
length used for DCA $\left(\boldsymbol{\lambda}_{\mathrm{exc}}=375 \mathrm{~nm}\right)$ should ensure a more selective excitation of the photooxidant.
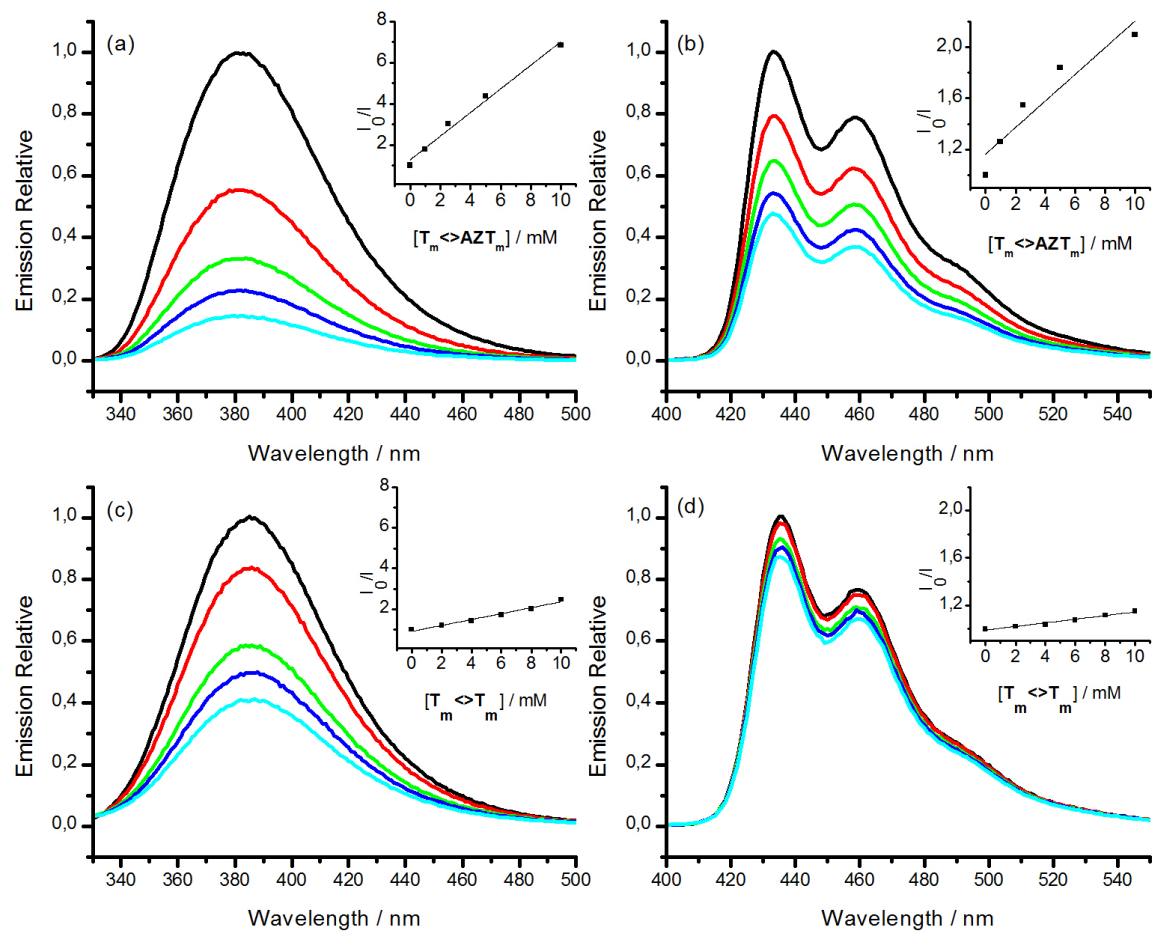

Figure 4.4. Fluorescence emission spectra for (a, c) DCN $\left(\lambda_{\text {exc }}=310 \mathrm{~nm}\right)$; (b,d) DCA $\left(\lambda_{\text {exc }}=375 \mathrm{~nm}\right)$; in the presence of increasing amounts of $\mathbf{T}_{\mathrm{m}}<>\mathbf{A Z} \mathbf{T}_{\mathrm{m}}$ (top) and $\mathbf{T}_{\mathrm{m}}<>\mathbf{T}_{\mathrm{m}}$ (bottom) from 0 to $10 \mathrm{mM}$.

To overcome this drawback and to evaluate whether a dynamic quenching is taking place, time-resolved fluorescence studies were performed (Figure 4.5). For the photooxidation process, a bimolecular quenching rate constant of ca. $7.8 \times 10^{9} \mathrm{M}^{-1} \mathrm{~s}^{-1}$ was obtained for the quenching of DCN and DCA by $\mathbf{T}_{\mathrm{m}}<>\mathbf{A Z T}_{\mathrm{m}}$. As expected, lower values $\left(\mathrm{k}_{\mathrm{q}}\right.$ of 3.0 and $\left.<0.5 \times 10^{9} \mathrm{M}^{-1} \mathrm{~s}^{-1}\right)$ were determined with the cyclobutane derivatives as deactivating species (Table 4.1). This revealed a more difficult oxidation 
of this compound by comparison with $\mathbf{T}_{\mathrm{m}}<>\mathbf{A Z} \mathbf{T}_{\mathrm{m}}$, which suggested that the azaU moiety plays a role in the photooxidative process.
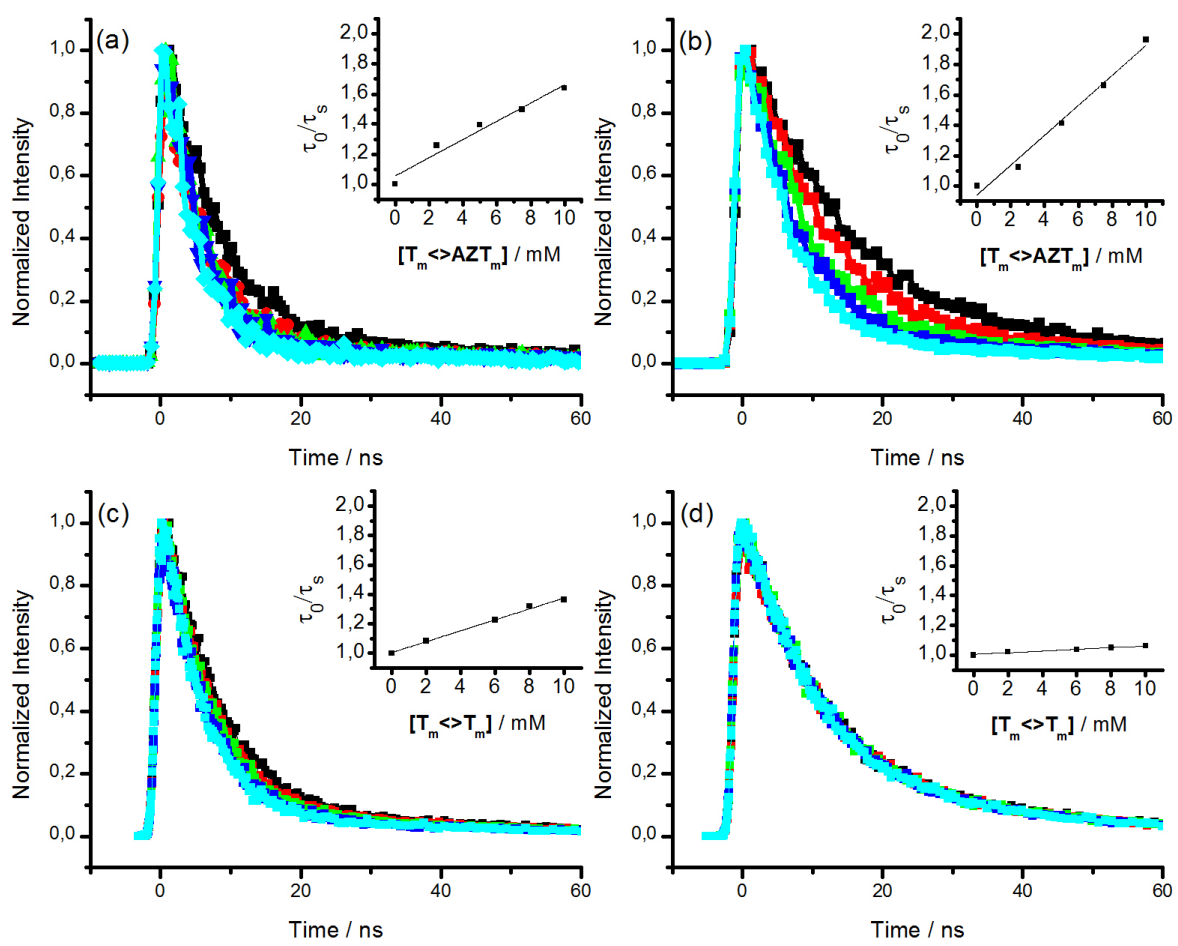

Figure 4.5. Fluorescence kinetic traces and their corresponding Stern-Volmer plot obtained for (a) DCN $\left(\lambda_{\text {exc }}=310 \mathrm{~nm}\right)$ and (b) DCA $\left(\lambda_{\text {exc }}=375 \mathrm{~nm}\right)$ in the presence of increasing amounts of $\mathbf{T}_{\mathrm{m}}<>\mathbf{A Z Z} \mathbf{T}_{\mathrm{m}}$ (from 0 to $10 \mathrm{mM}$ ); (c) DCN $\left(\boldsymbol{\lambda}_{\text {exc }}=310\right.$ $\mathrm{nm})$ and $(\mathrm{d})$ DCA $\left(\boldsymbol{\lambda}_{\mathrm{exc}}=375 \mathrm{~nm}\right)$ in the presence of increasing amounts of $\mathbf{T}_{\mathrm{m}}<>\mathbf{T}_{\mathrm{m}}$ (from 0 to $10 \mathrm{mM}$ ). 
Table 4.1. Reduction potential in the singlet excited state $\left(\mathrm{E}_{\text {red }}^{\mathrm{D}^{*}}\right)$ of the selected PS, and bimolecular rate constant $\left(\mathrm{k}_{\mathrm{q}}\right)$ for the quenching of the PS by $\mathbf{T}_{\mathrm{m}}<>\mathbf{A Z} \mathbf{T}_{\mathrm{m}}$ or $\mathbf{T}_{\mathrm{m}}<>\mathbf{T}_{\mathrm{m}}$ determined by time-resolved fluorescence.

\begin{tabular}{ccccc}
\hline PS & $\boldsymbol{\tau} / \mathbf{n s}$ & $\begin{array}{c}\mathbf{E}_{\text {red }}^{\mathbf{D}^{*}} / \mathbf{V} \mathbf{v s .} \\
\mathbf{A g} / \mathbf{A g C l}\end{array}$ & \multicolumn{2}{c}{$\mathbf{k}_{\mathrm{q}} / \mathbf{x} \mathbf{1 0} \mathbf{~ M}^{-1} \mathbf{s}^{-1}$} \\
\hline & & & $\mathbf{T}_{\mathrm{m}}<>\mathbf{A Z T}_{\mathrm{m}}$ & $\mathbf{T}_{\mathrm{m}}<>\mathbf{T}_{\mathrm{m}}$ \\
DCN & 7.5 & 2.4 & 7.8 & 3.0 \\
DCA & 12.5 & 1.8 & 7.8 & $<0.5$ \\
\hline
\end{tabular}

In order to support that the presence of azaU has an influence on the oxidative azetidine splitting, steady-state and time-resolved fluorescence experiments were performed with cis and trans $\mathbf{D C H}<>\mathbf{A Z T}_{\mathrm{m}}$ azetidines. As anticipated, the photooxidant emissions suffered an important quenching for both azetidines although a more efficient quenching was observed for the cis isomer (Figure 4.6). Moreover, the singlet lifetime of all these photooxidants was shortened in the presence of the azetidine derivatives as shown in Figure 4.7. The bimolecular quenching rate constants $\left(\mathrm{k}_{\mathrm{q}}\right.$, Table 4.2) were determined by Stern-Volmer plot using the data obtained by time-resolved fluorescence. ${ }^{21} \mathrm{~A}$ remarkable difference of quenching efficiency was observed between cis and trans (see $\mathrm{k}_{\mathrm{q}}$, Table 4.2) as shown for steady-state fluorescence (Figure 4.6), with similar $\mathrm{k}_{\mathrm{q}}$ values than for $\mathbf{T}_{\mathrm{m}}<>\mathbf{A Z} \mathbf{T}_{\mathrm{m}}$ suggesting a close value of their oxidation potentials. 

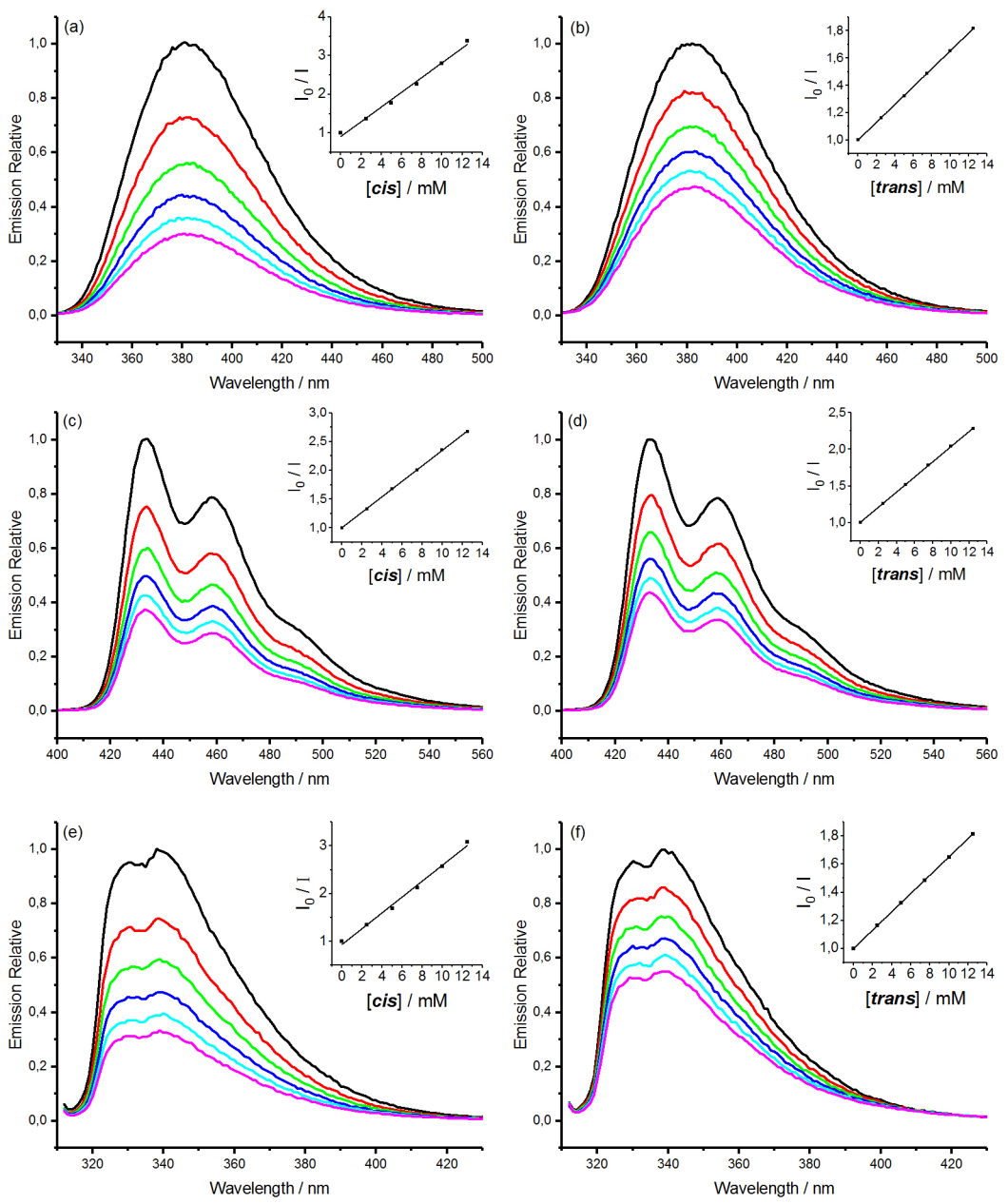

Figure 4.6. Fluorescence emission spectra for $(\mathrm{a}, \mathrm{b}) \mathrm{DCN}\left(\boldsymbol{\lambda}_{\mathrm{exc}}=310 \mathrm{~nm}\right) ;(\mathrm{c}, \mathrm{d})$ DCA $\left(\lambda_{\text {exc }}=375 \mathrm{~nm}\right) ;(\mathrm{e}, \mathrm{f}) \mathrm{CNN}\left(\lambda_{\mathrm{exc}}=310 \mathrm{~nm}\right)$ in the presence of increasing amounts of cis (left panel) and trans (right panel) $\mathbf{D C H}<>\mathbf{A Z T}_{\mathrm{m}}$ from 0 to $12.5 \mathrm{mM}$. 

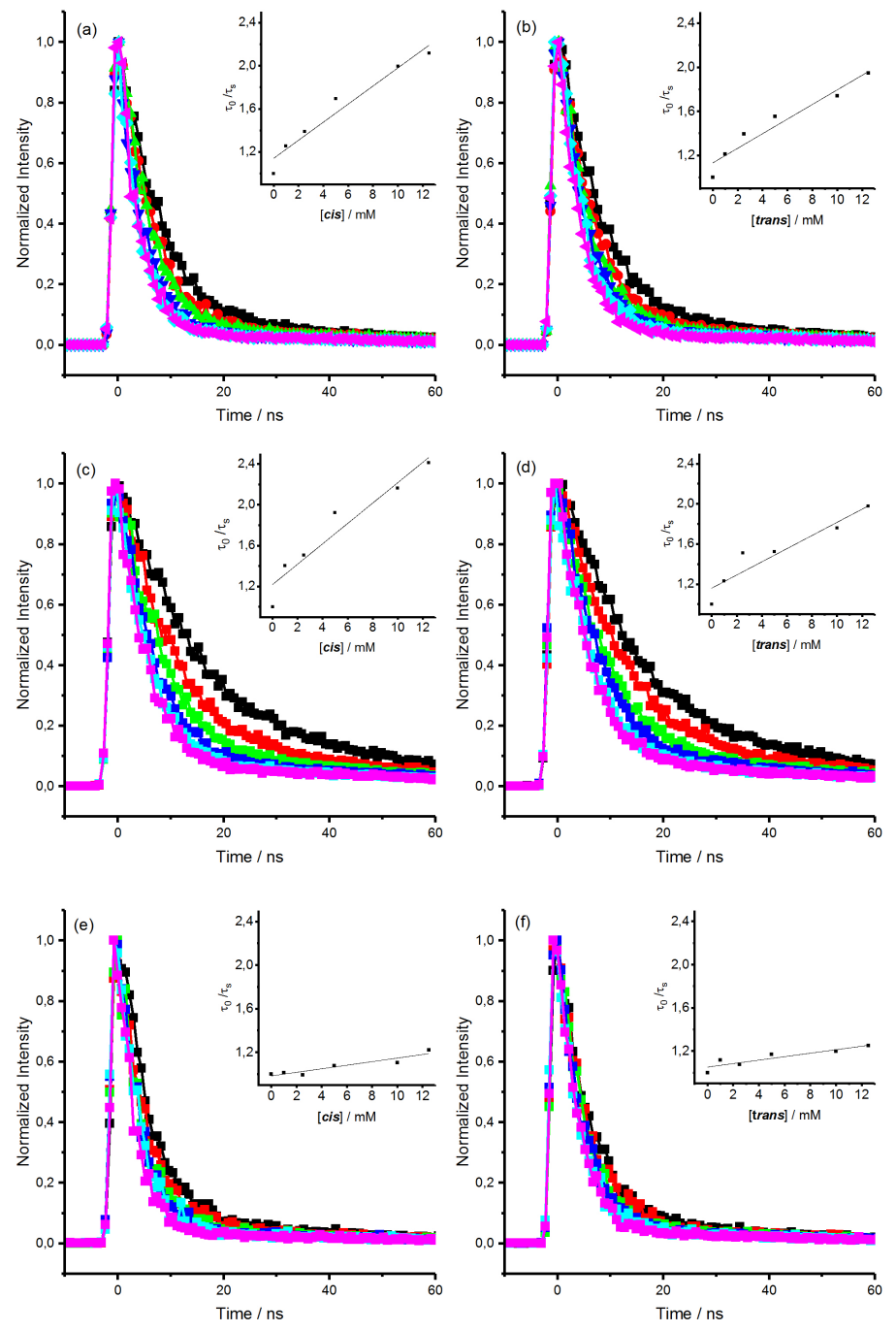

Figure 4.7. Fluorescence kinetic traces and their corresponding Stern-Volmer plot obtained for $(\mathrm{a}, \mathrm{b}) \operatorname{DCN}\left(\lambda_{\text {exc }}=310 \mathrm{~nm}\right)$; $(\mathrm{c}, \mathrm{d})$ DCA $\left(\boldsymbol{\lambda}_{\mathrm{exc}}=375 \mathrm{~nm}\right) ;(\mathrm{e}, \mathrm{f}) \mathrm{CNN}$ $\left(\lambda_{\text {exc }}=310 \mathrm{~nm}\right)$ in the presence of increasing amounts of cis (left panel) and trans (right panel) $\mathbf{D C H}<>\mathbf{A Z T}_{\mathrm{m}}$ from 0 to $12.5 \mathrm{mM}$. 
Chapter 4: Study of Azetidines Cycloreversion by Oxidative Electron Transfer

The two isomers have the same bonds and connectivity; however, they differ in the arrangement of the dihydroazauracil and cyclohexane rings. A similar stereoselectivity has previously been reported for photoreduction of the cis-syn and trans-syn diastereoisomers of cyclobutane dimethylthymine dimer. ${ }^{22}$ The more difficult reduction of the cis-syn isomer was attributed to a stereoelectronic effect as the formed anion radical suffers an unfavorable charge-dipole interaction with the carbonyl in $\mathrm{C}_{4}$ of the other pyrimidine ring. Nevertheless, this explanation cannot be extended to our cis and trans isomers as the cyclohexane ring does not provide pronounced electronic effects, and the most likely explanation relies on different constraints of the azetidine ring.

Table 4.2. Photophysical properties of the selected photosensitizers and their quenching rate constants by cis and trans $\mathbf{D C H}<>\mathbf{A Z} \mathbf{T}_{\mathrm{m}}$ determined by timeresolved fluorescence.

\begin{tabular}{ccccc}
\hline PS & $\begin{array}{c}\mathbf{E}^{*} / \mathbf{k J} \\
\text { mol- }^{-}\end{array}$ & $\begin{array}{c}\mathbf{E}_{\text {red }}^{\mathrm{D}^{*}} / \mathbf{V} \mathbf{v s .} \\
\mathbf{A g} / \mathbf{A g C l}\end{array}$ & \multicolumn{2}{c}{$\mathbf{k}_{\mathrm{q}} / \mathbf{\times 1 0 ^ { 9 }} \mathbf{M}^{-1} \mathbf{s}^{-1}$} \\
\hline & & & cis & trans \\
DCN & 362 & 2.4 & 10 & 7.7 \\
DCA & 276 & 1.8 & 6.4 & 4.6 \\
\hline
\end{tabular}

According to the Rehm-Weller equation, the quenching process was more efficient as $\mathrm{E}_{\text {red }}^{\mathrm{D}^{*}}$ increased, which supports an electron transfer mechanism for quenching. Indeed, singlet-singlet energy transfer can be ruled out because the absorption bands of $\mathbf{T}_{\mathrm{m}}<>\mathbf{A Z T} \mathbf{T}_{\mathrm{m}}, \mathbf{T}_{\mathrm{m}}<>\mathbf{T}_{\mathrm{m}}$ and $\mathbf{D C H}<>\mathbf{A Z} \mathbf{T}_{\mathrm{m}}$ compounds are below $300 \mathrm{~nm}$. Thus, their singlet energies ( $\left.\mathrm{E}^{*}\right)$ can be safely estimated to be $>400 \mathrm{~kJ} \mathrm{~mol}^{-1}$, that is more than $25 \mathrm{~kJ} \mathrm{~mol}^{-1}$ higher than energy of the highest $\mathrm{E}^{*}$ of the selected photosensitizer (Table 4.2). 


\subsubsection{Electrochemical measurements}

The different behavior observed for $\mathbf{T}_{\mathrm{m}}<>\mathbf{A Z} \mathbf{T}_{\mathrm{m}}$ and $\mathbf{D C H}<>\mathbf{A Z} \mathbf{T}_{\mathrm{m}}$ in comparison with $\mathbf{T}_{\mathrm{m}}<>\mathbf{T}_{\mathrm{m}}$ must be in connection with their oxidation potential. Thus, their electrochemical properties were studied by cyclic voltammetry as shown in Figure 4.8. According to the electron removal, two irreversible waves were observed for the dyad $\mathbf{T}_{\mathrm{m}}<>\mathbf{A Z} \mathbf{T}_{\mathrm{m}}$, the first one with a peak potential $\mathrm{E}_{\mathrm{p}}(1)$ at ca. $1.34 \mathrm{~V}$ and the second one $\mathrm{E}_{\mathrm{p}}(2)$ at ca. $1.93 \mathrm{~V}$. Interestingly, cis $\mathbf{D C H}<>\mathbf{A Z T} \mathbf{T}_{\mathrm{m}}$ showed a single wave at $1.30 \mathrm{~V}$, which is really close to the first $\mathrm{E}_{\mathrm{p}}$ of $\mathbf{T}_{\mathrm{m}}<>\mathbf{A Z} \mathbf{T}_{\mathrm{m}}$, and supports the similar $\mathrm{k}_{\mathrm{q}}$ obtained by fluorescence experiments. However, a more positive value at $1.55 \mathrm{~V}$ was observed for trans $\mathbf{D C H}<>\mathbf{A Z} \mathbf{T}_{\mathrm{m}}$, which means that the oxidation process is more difficult for trans than for $c$ is as reflected by fluorescence experiments.
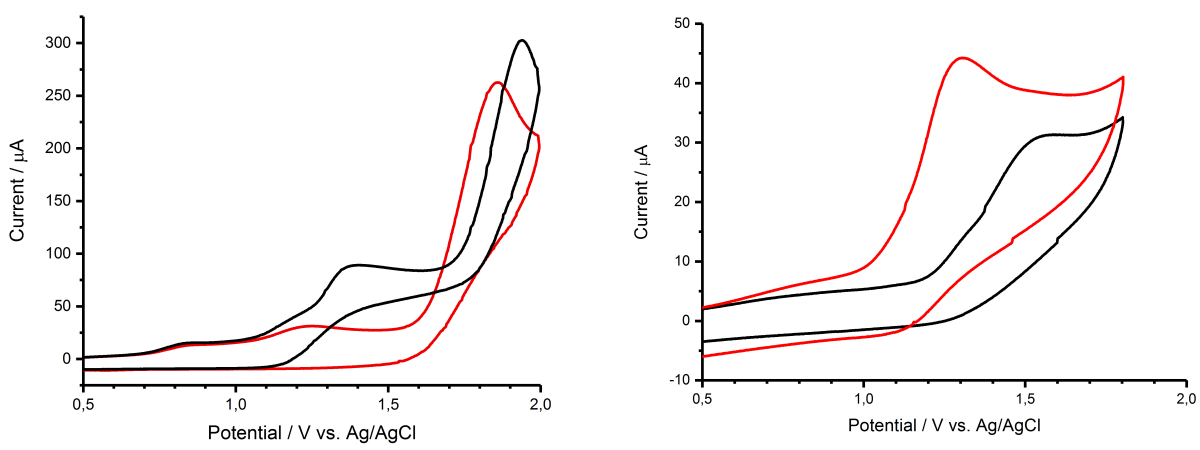

Figure 4.8. Cyclic voltammograms of: (left) $\mathbf{T}_{\mathrm{m}}<>\mathbf{A Z} \mathbf{T}_{\mathrm{m}}$ (black line, $2 \mathrm{mM}$ ) and $\mathbf{T}_{\mathrm{m}}<>\mathbf{T}_{\mathrm{m}}$ (red line, $2 \mathrm{mM}$ ) and (right) cis (red line, $1 \mathrm{mM}$ ) and trans (black line, 1 $\mathrm{mM}) \mathbf{D C H}<>\mathbf{A Z T}_{\mathrm{m}}$ in $\mathrm{N}_{2}$-purged acetonitrile using $0.1 \mathrm{M} \mathrm{n}-\mathrm{Bu}_{4} \mathrm{NClO}_{4}$ as electrolyte. Scan rate: 0.1 V.s.

The second peak of $\mathbf{T}_{\mathrm{m}}<>\mathbf{A Z T}_{\mathrm{m}}$ is similar to that observed for $\mathbf{T}_{\mathrm{m}}$-azaU $\mathbf{U}_{\mathrm{m}}$ oxidation (Figure 4.9), which indicates that the first wave leads to the cleavage of the dimer and formation of $\mathbf{T}_{\mathrm{m}}-\mathbf{a z a} \mathbf{U}_{\mathrm{m}} \cdot{ }^{23}$ This behavior is similar to that described for 
oxetanes or thietanes, where removal of one electron leads to cleavage of the heterocyclic 4-membered ring. ${ }^{24-25}$

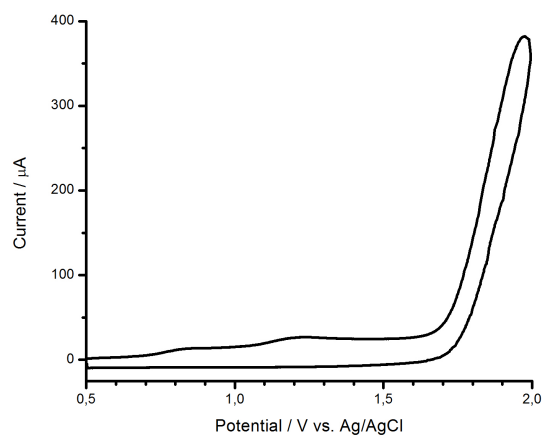

Figure 4.9. Cyclic voltammogram of azaU- $\mathbf{T}_{\mathrm{m}}(2 \mathrm{mM})$ in $\mathrm{N}_{2}$-purged acetonitrile using $0.1 \mathrm{M} \mathrm{n}-\mathrm{Bu}_{4} \mathrm{NClO}_{4}$ as electrolyte. Scan rate: 0.1 V.s.

Concerning, $\mathbf{T}_{\mathrm{m}}<>\mathbf{T}_{\mathrm{m}}$, a single irreversible wave was observed with an $\mathrm{E}_{\mathrm{p}}$ of $1.85 \mathrm{~V}$ (Figure 4.8), which is in agreement with its more difficult oxidation than that of the other compounds, and with the previously reported value of $1.83 \mathrm{~V}$ (vs. $\mathrm{Ag} / \mathrm{AgCl}) .{ }^{26}$ Altogether, these results show that there is an important stabilization of the radical cations when a nitrogen atom is part of the four-membered ring as for $\mathbf{T}_{\mathrm{m}}<>\mathbf{A Z} \mathbf{T}_{\mathrm{m}}$ and $\mathbf{D C H}<>\mathbf{A Z} \mathbf{T}_{\mathrm{m}}$, which are more easily oxidizable than $\mathbf{T}_{\mathrm{m}}<>\mathbf{T}_{\mathrm{m}}$.

\subsubsection{Photocycloreversion for $\mathrm{T}_{\mathrm{m}}<>\mathrm{AZT}_{\mathrm{m}}$ model}

The photocycloreversion of cyclobutane thymine dimers in the presence of compounds acting as oxidants in their excited states has been largely reported in the literature (see for example refs $20,27-28$ ). Hence, photooxidation of $\mathbf{T}_{\mathrm{m}}<>\mathbf{A Z Z} \mathbf{T}_{\mathrm{m}}$ was attempted in the presence of DCN as the electron acceptor and analyzed by HPLC to determine the photoproducts. A deaerated acetonitrile solution of $\mathbf{T}_{\mathrm{m}}<>\mathbf{A Z} \mathbf{T}_{\mathrm{m}}(1.5$ $\mathrm{mM}$ ) was irradiated at $330 \mathrm{~nm}$ in the presence of DCN (4 mM) for $100 \mathrm{~min}$. As shown in Figure 4.10, under these conditions the azetidine was split giving rise to the 
dyad $\mathbf{T}_{\mathrm{m}}-\mathbf{a z a} \mathbf{U}_{\mathrm{m}}$. However, a quantitative formation of $\mathbf{T}_{\mathrm{m}}-\mathbf{a z a} \mathbf{U}_{\mathrm{m}}$ was not observed (Figure 4.10 , inset). This can be attributed to the highly reactive pyrimidine radical cations formed, which after deprotonation, hydration and addition of $\mathrm{O}_{2}$ lead to the formation of secondary products. ${ }^{29}$ Indeed, a similar behavior has been described in the literature for the photocycloreversion of cyclobutane thymine (and cytosine) dimers in the presence of electron acceptors. ${ }^{4}$ In addition, photocycloreversion of both isomers of $\mathbf{D C H}<>\mathbf{A Z T}_{\mathbf{m}}$ with DCN did not lead to methylated azaU and cyclohexene. This can be due to (i) the formation of side products from the radical cation azaU or (ii) a different splitting pathway of the azetidine heterocycle to generate a twelvemembered ring.

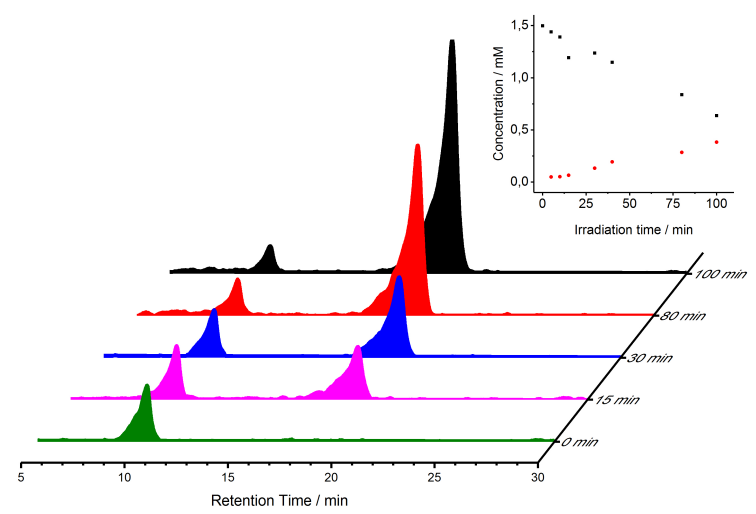

Figure 4.10. HPLC chromatograms, monitored at $\lambda=220 \mathrm{~nm}$, of the photooxidative cycloreversion of $\mathbf{T}_{\mathrm{m}}<>\mathbf{A Z} \mathbf{T}_{\mathrm{m}}$ (at retention time of ca. $10 \mathrm{~min}$ ) and $\mathbf{T}_{\mathrm{m}}-\mathbf{a z a} \mathbf{U}_{\mathrm{m}}$ (at retention time of ca. $20 \mathrm{~min}$ ) in the presence of DCN under monochromatic irradiation at $330 \mathrm{~nm}$. Inset: Time course of the photoreaction $\left(\mathbf{T}_{\mathrm{m}}<>\mathbf{A Z} \mathbf{T}_{\mathrm{m}}\right.$, squares and $\mathbf{T}_{\mathrm{m}}$-azaU $\mathbf{m}_{\mathrm{m}}$ circles). 
Chapter 4: Study of Azetidines Cycloreversion by Oxidative Electron Transfer

\subsubsection{Quantum-chemistry determination of the photooxidative properties for $\mathrm{T}_{\mathrm{m}}<>\mathrm{AZ} \mathrm{T}_{\mathrm{m}}$ model}

The study was performed in collaboration with the research group of Dr. RocaSanjúan (University of Valencia). To gain insight into the energetics involved in the removal of one electron in the azetidine and cyclobutane derivatives, the ionization potentials (IPs) were computed for the $N$-demethylated compounds $\mathbf{T}<>$ AZT and $\mathbf{T}<>\mathbf{T}$ with the density functional theory (DFT) method, the M06-2X functional and the $6-31++\mathrm{G}(\mathrm{d}, \mathrm{p})$ basis set. Benchmark calculations on the thymine nucleobase compared with high-level complete-active-space self-consistent field second-order perturbation theory (CASPT2) results indicate an accurate performance of the DFT/M062X method for the vertical and adiabatic IPs (VIPs and AIPs). Table 4.3 compiles the computed gas phase and acetonitrile solution values, which also allows an estimation of the solvent effect. As expected, solvation has an important influence on the IP because it stabilizes to a higher extent the ionic states as compared to the neutral state. Moreover, Table 3.3 shows that EAs of methylated and demethylated compounds of T<>AZT differ slightly, thus theoretical studies with demethylated compound give us reliable results as for methylated compounds. Regarding IPs, Table 4.3 indicates significantly lower IPs values for $\mathbf{T}<>\mathbf{A Z T}$ than for $\mathbf{T}<>\mathbf{T}$. This allows us to interpret the fact that higher experimental quenching rates $\left(\mathrm{k}_{\mathrm{q}}\right)$ were measured for $\mathbf{T}<>\mathbf{A Z T}$ than for $\mathbf{T}<>\mathbf{T}$ in the case of photooxidants (DCN and DCA) (Table 4.2). 
Table 4.3. Vertical (V) and adiabatic (A) ionization potentials (IPs) in eV (kcal mol-1 $^{-1}$ within parentheses) for $\mathbf{T}<>\mathbf{A Z T}, \mathbf{T}_{\mathrm{m}}<>\mathbf{A Z} \mathbf{T}_{\mathrm{m}}$ and $\mathbf{T}<>\mathbf{T}$ in the gas phase and in acetonitrile computed with the DFT/M06-2X method and the 6-31++G** basis set.

\begin{tabular}{lcc}
\hline & VIP & AIP \\
\hline T<>AZT & & \\
Gas phase & $9.02(207.9)$ & $8.06(185.8)$ \\
Acetonitrile & $7.14(164.7)$ & $6.23(143.7)$ \\
$\mathbf{T}_{\mathbf{m}}<>$ AZT $_{\mathbf{m}}$ & & \\
Gas phase & $8.77(202.2)$ & $7.86(181.3)$ \\
Acetonitrile & $7.04(162.2)$ & $6.14(141.7$ \\
$\mathbf{T}<>\mathbf{T}$ & & \\
Gas phase & $9.30(214.5)$ & $8.64(199.2)$ \\
Acetonitrile & $7.57(174.6)$ & $6.92(159.6)$ \\
\hline
\end{tabular}

\subsubsection{Decompostition mechanism of $\mathrm{T}<>\mathrm{AZT}$ radical cation}

For the photooxidation, the four-membered ring is preserved in the cationic structure, in contrast to the findings obtained for the anion, which was discussed in Chapter 3. The reaction mechanism of $\mathbf{T}<>\mathbf{A Z Z} \mathbf{T}^{\bullet+}$ implies a two-step N-C and C-C bond breaking, in which the first cleavage $(\mathrm{N}-\mathrm{C})$ requires ca. $7.5 \mathrm{kcal} \mathrm{mol}^{-1}$ and gives rise to a flat energy profile, although with no stable structure (Figure 4.11). In such region, the carbon atom of the broken bond becomes a carbocation. It can be seen in the gas-phase with the complete-active space selfconsistent field (CASSCF) spindensity representations of Figure 4.12 that the unpaired electron is localized over the nitrogen atom and to some extent also over the adjacent nitrogen atom. The second step in the mechanism corresponds to the $\mathrm{C}-\mathrm{C}$ bond breaking and increases the energy barrier for the overall process up to $12.5 \mathrm{kcal} \mathrm{mol}^{-1}$. Therefore, these data showed 
that the oxidative mechanism of $\mathbf{T}_{\mathbf{m}}<>\mathbf{A} \mathbf{Z} \mathbf{T}_{\mathbf{m}}$ occurs through the pathway a in Scheme 4.3.

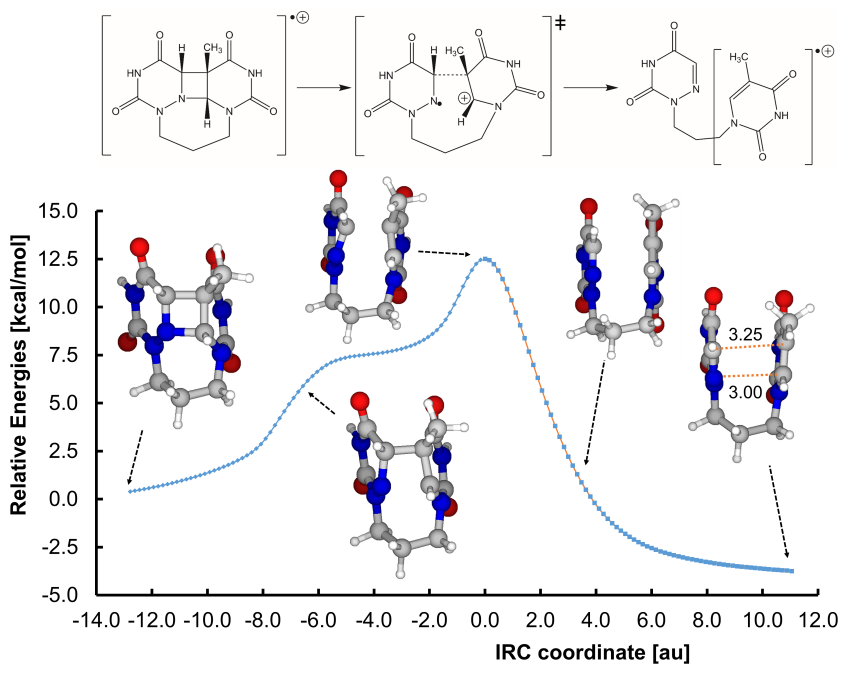

Figure 4.11. Chemical structure of relevant points (top) and energy profile (bottom) for the C-N and C-C bond breakings of $\mathbf{T}<>\mathbf{A Z T}^{\bullet+}$ obtained with MEP computational strategy.
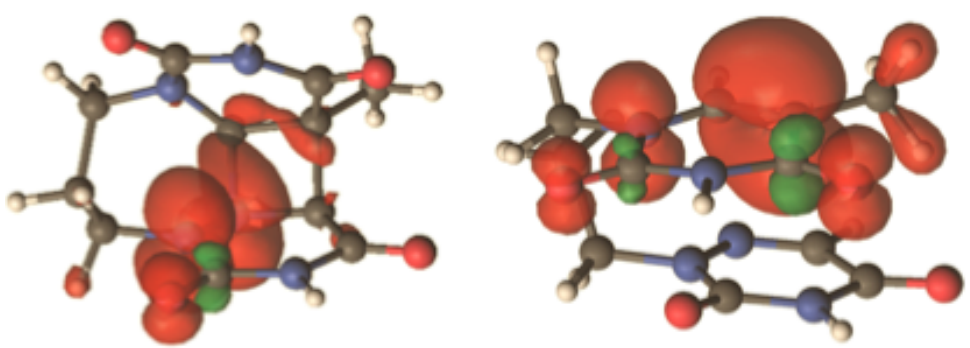

Figure 4.12. CASSCF spin-density representations for the $\mathbf{T}_{\mathrm{m}}<>\mathbf{A Z} \mathbf{T}_{\mathrm{m}}$ radical cation systems at the reductant (left) and product (right) geometries. Red $=$ excess of spin density, Green = defect of spin density. 
Globally, the in-vacuo mechanism described above implies energy barriers lower than $13 \mathrm{kcal} \mathrm{mol}^{-1}$ for cation system. To further improve the description, we have focused on the relevant points for the present mechanism (reactants, TSs and products) and we have determined several thermochemical magnitudes using the highly accurate CASPT2 method. Results are compiled in Table 4.4. For $\mathbf{T}_{\mathrm{m}}<>\mathbf{A Z \mathbf { Z T } _ { \mathrm { m } }}{ }^{\bullet+}$, both methods differ in the sign of $\Delta \mathrm{E}$; however, the values are around zero. Regarding the solvent effects, in contrast to the redox properties determined in the previous section, no significant changes are observed for the $\mathbf{T}_{\mathrm{m}}<>\mathbf{A Z} \mathbf{T}_{\mathrm{m}}{ }^{\bullet+}$ cycloreversion mechanism. This is due to the fact that along the ring opening reactions the total charge is preserved. The ZPVE correction gives rise to lower $\Delta$ Es as a consequence of the bond breakings occurring along the reactions. The same behavior is observed for $\Delta G$, which reflects the entropy increase in the decomposition process.

Table 4.4. Computed energy differences between products and the reactants $\left(\Delta \mathrm{E}, \Delta \mathrm{E}_{0}\right.$ and $\left.\Delta \mathrm{G}\right)$ and activation energies $\left(\Delta \mathrm{E}^{\ddagger}, \Delta \mathrm{E}_{0}^{\ddagger}\right.$ and $\left.\Delta \mathrm{G}^{\ddagger}\right)$ for the cycloreversion of the $\mathbf{T}<>$ AZT radical cation. Energies in $\mathrm{kcal}^{\mathrm{mol}}{ }^{-1}$.

\begin{tabular}{lcc}
\hline Methodology & \multicolumn{2}{c}{ AZT $^{\mathbf{0}^{+}}$} \\
\hline \multirow{2}{*}{ M06-2X } & $\Delta \mathrm{E}$ & $\Delta \mathrm{E}^{\ddagger}$ \\
\cline { 2 - 3 } CASPT2//M06-2X & -4.89 & 12.52 \\
PCM-M06-2X & 2.21 & 14.77 \\
& -3.09 & 13.52 \\
M06-2X & $\Delta \mathrm{E}_{0}$ & $\Delta \mathrm{E}_{0}^{\ddagger}$ \\
\cline { 2 - 3 } CASPT2//M06-2X & -7.36 & 10.73 \\
& -0.26 & 12.98 \\
M06-2X & $\Delta \mathrm{G}$ & $\Delta \mathrm{G}^{\ddagger}$ \\
\cline { 2 - 3 } CASPT2//M06-2X & -8.99 & 11.47 \\
\hline
\end{tabular}


Chapter 4: Study of Azetidines Cycloreversion by Oxidative Electron Transfer

\subsection{Conclusions}

The photooxidative cycloreversion of azetidine models $\mathbf{T}_{\mathrm{m}}<>\mathbf{A} \mathbf{Z} \mathbf{T}_{\mathrm{m}}$ has been addressed through a multidisciplinary study including spectroscopic, electrochemical and analytical experiments complemented by computational chemistry. In a first stage, the electron accepting capabilities of this four-membered heterocycle have been established by means of photooxidant singlet excited state quenching. In this context, comparison of the bimolecular rate constants obtained for $\mathbf{T}_{\mathrm{m}}<>\mathbf{A Z} \mathbf{T}_{\mathrm{m}}$ with $\mathbf{D C H}<>\mathbf{A Z T} \mathbf{T}_{\mathrm{m}}$ and $\mathbf{T}_{\mathrm{m}}<>\mathbf{T}_{\mathrm{m}}$ has revealed that the presence of the nitrogen atom in the ring affects its oxidation. The electrochemical experiments fully support these redox features as close oxidation peaks have been registered for $\mathbf{T}_{\mathrm{m}}<>\mathbf{A Z} \mathbf{T}_{\mathrm{m}}$ and $\mathbf{D C H}<>\mathbf{A Z T}_{\mathrm{m}}$, by contrast their oxidation waves are differing in $0.5 \mathrm{~V}$ with $\mathbf{T}_{\mathrm{m}}<>\mathbf{T}_{\mathrm{m}}$. This variation has also been corroborated by DFT quantum chemistry, which has determined different ionization potentials of ca. 6.23 and $6.92 \mathrm{eV}$ for $\mathbf{T}_{\mathrm{m}}<>\mathbf{A Z} \mathbf{T}_{\mathrm{m}}$ and $\mathbf{T}_{\mathrm{m}}<>\mathbf{T}_{\mathrm{m}}$, respectively. Concerning the fate of the formed $\mathbf{T}_{\mathrm{m}}<>\mathbf{A Z T}_{\mathrm{m}}$ ion radicals, the cycloreversion of the ring to restore thymine and 6azauracil bases has been evidenced by steady state photolysis followed by HPLC analysis. The photooxidative version involves a bond cleavage of $\mathrm{C}-\mathrm{N}$ followed by $\mathrm{C}-\mathrm{C}$, with a similar energy barrier and a final radical cation on the thymine nucleobase. Altogether, these experimental and theoretical findings represent an important advance in the photoinduced cycloreversion of azetidines by electron transfer. 


\subsection{References}

1. Sancar, A., Structure and function of DNA photolyase and cryptochrome blue-light photoreceptors. Chem. Rev. 2003, 103, 2203-2207.

2. Dandliker, P. J.; Holmlin, R. E.; Barton, J. L., Oxidative thymine dimer repair in the DNA helix. Science 1997, 275, 1465-1468.

3. Roth, H. D.; Lamola, A. A., Cleavage of thymine dimers sensitized by quinones. Chemically induced dynamic nuclear polarization in radical ions. J. Am. Chem. Soc. 1972, 94, 1013-1014.

4. Fenick, D. J.; Carr, H. S.; Falvey, D. E., Synthesis and photochemical cleavage of cis-syn pyrimidine cyclobutane dimer analogs. J. Org. Chem. 1995, 60, 624-631.

5. Sasson, S.; Elad, D., Photosensitized monomerization of 1,3-dimethyluracil photodimers. J. Org. Chem. 1972, 37, 3164-3167.

6. Hartman, R. F.; Rose, S. D.; Pouwels, P. J. W.; Kaptein, R., Flavin-sensitized photochemically induced dynamic nuclear polarization detection of pyrimidine dimer radicals. Photochem. Photobiol. 1992, 56, 305-310.

7. Rosenthal, I.; Rao, M. M., Transition metal-ion photosensitized monomerization of pyrimidine dimers. Biochem. Biophys. Acta 1975, 378, 165-168.

8. Hartman, R. F.; Rose, S. D., A possible chain reaction in photosensitized splitting of pyrimidine dimers by a protonated, oxidized flavin. J. Org. Chem. 1992, 57, 2302-2306.

9. Schmuck, C.; Wennemers, H., Highlights in Bioorganic Chemistry: Methods and Applications. 2005, 352-368.

10. Pouwels, J. W.; Kaptein, R.; Hartman, R. F.; Rose, S. D., Photo-CIDNP study of pyrimidine dimer splitting I: reactions involving pyrimidine radical cation intermediates. Photochem. Photobiol. 1995, 61, 563-574.

11. Austin, R.; McMordie, S.; Begley, T. P., Mechanistic studies on DNA photolyase. Secondary deuterium isotope effects on the cleavage of the uracil photodimer radical cation and anion. J. Am. Chem. Soc. 1992, 114, 1886-1887. 
12. Voityuk, A. A.; Rösch, N., A quantum chemical study of photoinduced DNA repair: on the splitting of pyrimidine model dimers initiated by electron transfer. $J$. Am. Chem. Soc. 1996, 118, 9750-9758.

13. Krüger, O.; Wille, U., Oxidative cleavage of a cyclobutane pyrimidine dimer by photochemically generated nitrate radicals. Org. Lett. 2001, 3, 1455-1458.

14. Young, T. N., R.; Rose, S. D., Photo-CIDNP detection of pyrimidine dimer radical cations in anthraquinone sulfonate-sensitized splitting. Photochem. Photobiol. 1990, 52, 661-668.

15. Wang, Y.; Gaspar, P. P.; Taylor, J. E., Quantum chemical study of the electron transfer-catalyzed splitting of oxetane and azetidine intermediates proposed in the photoenzymatic repair of (6-4) photoproduct of DNA. J. Am. Chem. Soc. 2000, 122, 5510-5519.

16. Nakabayashi, K.; Kojima, J.; Yasuda, T. M.; Shima, K., Organic photochemical reactions. XXVI. Photosensitized ring-cleavage reactions of 2,2diaryloxetanes by aromatic nitriles. Bull. Chem. Soc. Jpn. 1989, 62, 96-101.

17. Prakash, G.; Falvey, D. E., Model studies of the (6-4) photoproduct DNA photolyase: synthesis and photosensitized splitting of a thymine-5,6-oxetane. J. Am. Chem. Soc. 1995, 117, 11375-11376.

18. Miranda, M. A.; Izquierdo, M. A., Stepwise cycloreversion of oxetane radical cations with initial C-O bond cleavage. J. Am. Chem. Soc. 2002, 124, 6532-6533.

19. Andreu, I.; Delgado, J.; Espinós, A.; Pérez-Ruiz, R.; Jiménez, M. C.; Miranda, M. A., Cycloreversion of azetidines via oxidative electron transfer: steady-state and time-resolved studies. Org. Lett. 2008, 10, 5207-5210.

20. Pac, C.; Ohtsuki, T.; Shiota, Y.; Yanagida, S.; Sakurai, H., Photochemical reactions of aromatic compounds. XLII. Photosensitized reactions of some selected diarylcyclobutanes by aromatic nitriles and chloranil. Implications of charge-transfer contributions on exciplex reactivities. Bull. Chem. Soc. Jpn 1986, 59, 1133-1139. 
21. Stern, O.; Volmer, M., Über die abklingungszeit der fluoreszenz. Physik. Z. 1919, 20, 183-188.

22. Scannell, M. P.; Fenick, D. J.; Yeh, S.; Falvey, D. E., Model studies of DNA photorepair: reduction potentials of thymine and cytosine cyclobutane dimers measured by fluorescence quenching. J. Am. Chem. Soc. 1997, 119, 1971-1977.

23. Boussicault, F.; Kruger, O.; Robert, M.; Wille, U., Dissociative electron transfer to and from pyrimidine cyclobutane dimers: An electrochemical study. Org. Biomol. Chem. 2004, 2, 2742-2750.

24. Boussicault, F.; Robert, M., Electrochemical approach to the repair of oxetanes mimicking DNA (6-4) photoproducts. J. Phys. Chem. B 2006, 110, 2198721993.

25. Boussicault, F.; Robert, M., Electron transfer in DNA and in DNA-related biological processes. Electrochemical insights. Chem. Rev. 2008, 108, 2622-2645.

26. Wenska, G.; Paszyc, S., Electron-acceptor-sensitized splitting of cyclobutanetype thymine dimers. J. Photochem. Photobiol. B: Biol. 1990, 8, 27-37.

27. Fenick, D. J.; Carr, H. S.; Falvey, D. E., Synthesis and photochemical cleavage of cis-syn pyrimidine cyclobutane dimer analogs. J. Org. Chem. 1996, 60, 624-631.

28. Pac, C.; Miyamoto, I.; Masaki, Y.; Furusho, S.; Yanagida, S.; Ohno, T.; Yoshimura, A., Chloranil-photosensitized monomerization of dimethylthymine cyclobutane dimers and effect of magnesium perchlorate. Photochem. Photobiol. 1990, 52, 973-979.

29. Cadet, J.; Vigny, P., In Bioorganic Photochemistry. H. Morrison, Ed. Wiley: New York 1990, 1-272. 


\section{Chapter 5:}

\section{Photorepair of Thymine Derived Cyclobutanes and Oxetanes by \\ 8-oxoGuanine}





\subsection{Introduction}

Over millions of years, nature has developed catalytic RNA enzymes denominated ribozymes that play an important role in the evolution of life via the 'RNA World' hypothesis. ${ }^{1}$ This hypothesis explains how RNA, which served as genome and as catalyst, passed its genomic role to DNA and its catalytic role to proteins. Indeed, numerous in vitro selection experiments have confirmed the concept that RNA can catalyze a wide range of chemical reactions such as ligation, hydrolysis, and $\mathrm{C}-\mathrm{C}$ bond formation. ${ }^{2}$ In this context, it would be interesting to investigate whether nucleic acid enzymes are capable of catalyzing photochemical reactions to repair the UV photoproducts in DNA as photolyases do. In 2004, this theory was supported by means of a deoxyribozyme formed by guanine bases in a quadruplex structure. ${ }^{3}$ Interestingly, this deoxyribozyme acts as an antenna for excitation at $305 \mathrm{~nm}$, wavelength longer than those absorbed by normal bases, and repairs efficiently cyclobutane dimers via an electron donation from an excited guanine base. Therefore, this work opened the door to investigate whether nucleic acids in their excited state are able to donate one electron to thymine dimers and repair them by analogy with CPD photolyase.

In 2007, the importance of $G$ adjacent bases in the formation of CPDs was investigated by steady-state photolysis of series of duplexes (18 nucleotides) containing a single TT central sequence flanked by purines or pyrimidines bases. ${ }^{4}$ Interestingly, lower levels of $\mathrm{T}<>\mathrm{T}$ were found when guanosine was adjacent to the cyclobutane dimer at the 5'-side. This purine effect could be explained by considering the fact that, among the DNA bases, $G$ has the lowest oxidation potential $\left(E_{o x}(G)=1.3 \mathrm{~V}\right.$ vs. NHE), and thus $G$ is the most easily oxidizable base. ${ }^{5}$ Therefore, by analogy with CPD photolyase, electron transfer from the excited guanosine to the TT dimer was proposed to lead to the formation of the TT dimer radical anion that would cleave to 
finally give rise to the initial $\mathrm{T}$ bases (Scheme 5.1). The overall process results in a decrease of the stationary level of CPD.

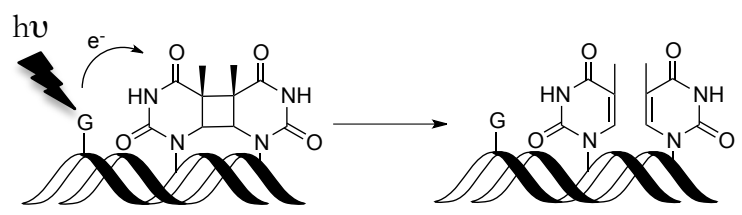

Scheme 5.1. Mechanistic pathway of CPD repair from excited guanine nucleobase by PET.

However, it was reported that the purine effect in the dimerization of TT at 302 $\mathrm{nm}$ was not the result of a purine-sensitized repair mechanism as previously mentioned because of the absence of purine absorption at this wavelength. ${ }^{6}$ It was thus proposed that it is the consequence of the pyrimidine excited state quenching by electron transfer from the flanking base, which would result in the formation of an exciplex that could deactivate to the ground state via nonradiative decay rather than exciplex fluorescence or TT dimer formation. In further support of exciplex mechanism, a lower photodimer yield was observed when $\mathrm{G}$ is located in the 5'-position relative to the dipyrimidine site. This points to a better stacking of purine-pyrimidine sequences compared to pyrimidine-purine sequences, which would facilitate electron transfer and exciplex formation. Moreover, the determination of quantum yields for thymine photodimerization containing a single T'T with flanking purines proved this purine effect, which was attributed to a combination of ground state donor-acceptor interactions between $\pi$-stacked purine and thymine bases and to an excited state exciplex formation. ${ }^{7}$

By contrast, femtosecond transient absorption experiments showed that electron transfer from excited $\mathrm{G}$ or $\mathrm{A}$ to $\mathrm{T}<>\mathrm{T}$ does no ocurr to a significant extent in singlestranded trinucleotides. ${ }^{8}$ However, this could be attributable to poor stacking between 
the electron donor and the CPD in these short and single stranded sequences. Thus, electron transfer may be too slow to compete with fast nonradioactive deactivation of the purine ${ }^{1} \pi \pi^{*}$ excited state, that has a lifetime of less than 1 ps. ${ }^{9}$

Recently, a new mechanism based on intrastrand excimer, in which the excitation is shared by two neighboring stacked bases, was proposed to repair $\mathrm{T}<>\mathrm{T}$ flanked by GA nucleotides through a charge transfer state. ${ }^{10}$ In this case, the excitation of $G$ induces an electron migration to a nearby A giving rise to the formation of the zwitterion $\mathrm{G}^{\bullet+} \mathrm{A}^{\bullet-}$ that has a lifetime long enough (300 ps) to donate an electron to the adjacent $\mathrm{T}<>\mathrm{T}$ lesion, which finally leads to the repaired thymine bases. By contrast, direct photoinduced repair via charge transfer from photoexcited $A$ or $G$ to adjacent $\mathrm{T}<>\mathrm{T}$ was negligible, which agrees with the previous femtosecond transient absorption investigation. ${ }^{8}$

In 2011, an unusual example was reported where a DNA lesion has the capacity to repair another one, the CPDs, imitating this way the function of the flavin cofactor in CPD photolyase mechanism. ${ }^{11-12}$ For small oligonucleotides, it has been shown that, after photoexcitation, the oxidized guanine, 8-oxoGuanine (OG), transfers an electron to an adjacent CPD lesion, which induces the splitting of the cyclobutane ring and regeneration of $O G$ and thymine bases after a subsequent back electron transfer.

Interesting properties make OG a better candidate for DNA-damage repair than G. Although $G$ has the lowest redox potential among the DNA bases ${ }^{5}$ and this value can be modulated in sequences with consecutive $G^{13}$, the $O G$ has a redox potential of ca. $0.5 \mathrm{~V}$ lower than that of $\mathrm{G}\left(\mathrm{E}_{\mathrm{ox}}=0.7 \mathrm{~V}\right.$ vs. NHE) and a red-shifted UV absorption with a band above $300 \mathrm{~nm}$ that allows selective excitation.

Since this research, the interest in the photophysical properties of OG has increased and, theoretical ${ }^{14-17}$ and ultrafast studies ${ }^{18-20}$ have been carried out in order to prove the ability of OG to act as an electron donor in the photoinduced electron transfer mechanism proposed for CPD repair. The lifetime of the excited state also 
plays an important role. In this context, it was found that the lifetime of first excited state of OG is of ca. 0.9 ps, i.e. twice longer than that of G. ${ }^{18}$ The ultrafast nonradiative process could avoid the occurrence of photochemical pathways and, thus, reduce CPD repair by PET despite favorable thermodynamics. However, the deactivation pathways may be different in the presence of base stacking and pairing. In this context, the UV excitation of a dinucleotide containing OG at 5' side of an adenine showed the formation of a radical ion pair with a lifetime of 60 ps that was assigned to an interbase charge transfer state, which lives much longer than the excited state of $\mathrm{A}$ or OG monomers. ${ }^{19}$ Therefore, this result proved the ability of OG to transfer an electron to a stacked adenine, mimicking thus the first step of the flavin cofactor $\mathrm{FADH}^{-}$involved in the repair by photolyase. In addition, this photoreactivation may be possible despite the short lifetime of the radical ion pair in DNA because the reductive splitting step of CPD has been shown to occur in less than 100 ps. ${ }^{21}$ Altogether, these results show that there is an ongoing discussion whether the excitation of a single purine nucleobase such as $G$ or OG promotes or not the repair of a neighboring $\mathrm{T}<>\mathrm{T}$ dimer through electron transfer.

Regarding model systems, there is a plenty of examples using CPD unit covalently linked to chromophores such as indole ${ }^{22}$, aryl amine ${ }^{23}$, methoxybenzene ${ }^{24}$, flavin ${ }^{25-27}$ or tryptophan ${ }^{28}$; but also using the oxetane unit ${ }^{27,29}$ as a model for the intermediate involved in 6-4PP repair. By contrast, no model systems of CPDs or oxetanes incorporating $O G$ or $G$ as electron donor have been reported. With this background, we considered to study the ability of OG and G covalently linked to an oxetane unit and to a thymine dimer to photoinduce the cycloreversion process. Firtsly, the splitting process of an oxetane model (10, Figure 5.1) attached to OG nucleoside was studied. Secondly, four models containing the CPD or oxetane unit were attached to OG or G nucleobases by a flexible linker $(15,16,20,21$, Figure 5.1) in order to study and compare the PET process between the electron donors, G and OG, and the electron acceptors CPD and oxetane. 

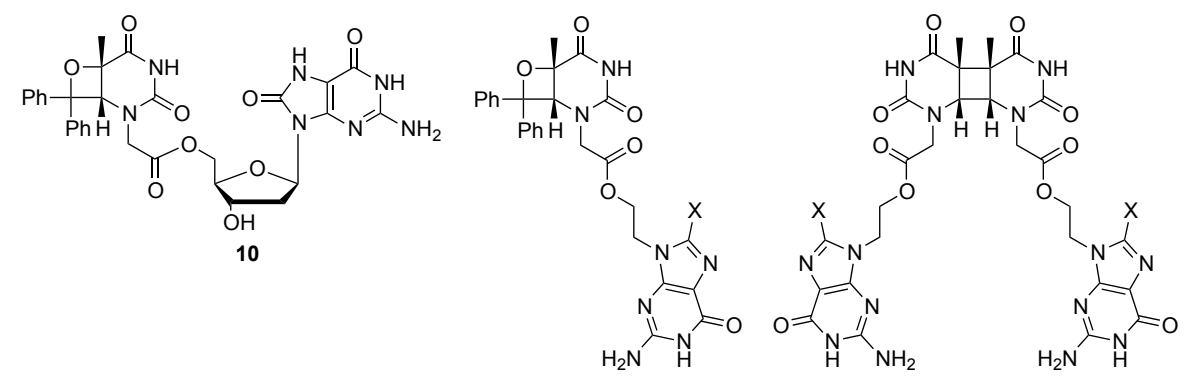

$$
\begin{aligned}
& \text { 15: } \mathrm{X}=\mathrm{OH} \\
& \text { 16: } \mathrm{X}=\mathrm{H}
\end{aligned}
$$$$
\begin{aligned}
& \text { 20: } \mathrm{X}=\mathrm{OH} \\
& \text { 21: } \mathrm{X}=\mathrm{H}
\end{aligned}
$$

Figure 5.1. Structure of the five models with oxetane $(10,15,16)$ and thymine dimer $(\mathbf{2 0}, \mathbf{2 1})$ attached to $O G$ or $G$ chromophores.

\subsection{Results and discussion}

\subsubsection{Synthesis}

Firstly, we decided to synthesize the model 10 (Figure 5.1) where OG nucleoside is covalently attached to an oxetane compound through the 5'-OH of the 2'deoxyribose. Previous thymine dyads designed in our group showed a strong intramolecular interaction between the thymine and a chromophore in the excited state when this chromophore is attached in the 5' position of the 2'-deoxyribose moiety. ${ }^{30}$ The five-step synthesis of the 3'-protected OG derivative 5, required for the synthesis of model compound 10, is shown in Scheme 5.2.

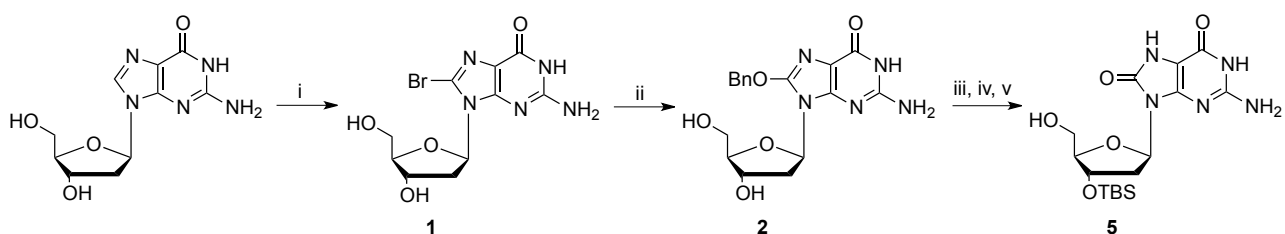

Scheme 5.2. Synthetic strategy to prepare 5. Reagents and conditions: (i) NBS, $\mathrm{MeCN} / \mathrm{H}_{2} \mathrm{O}$, rt, 45 min; (ii) $\mathrm{Na}, \mathrm{BnOH}, \mathrm{DMSO}, 65^{\circ} \mathrm{C}$, 24h; (iii) $1 \mathrm{M} \mathrm{HCl}, \mathrm{MeOH}$, rt, 1h; (iv) TBDMSCl, Im, DMF, rt, 24h; (v) TFA $/ \mathrm{H}_{2} \mathrm{O}, \mathrm{CH}_{2} \mathrm{Cl}_{2}, 0^{\circ} \mathrm{C}$, $4 \mathrm{~h}$. 
To obtain the OG moiety, 2'-deoxyguanosine was brominated at the $\mathrm{C} 8$ position to give 1 , this step was followed by a nucleophilic substitution with sodium benzylate leading to compound 2. The latter is highly unstable in acidic conditions and yields OG nucleoside 3 upon stirring with $1 \mathrm{M} \mathrm{HCl}$ for $1 \mathrm{~h}$. Then, the hydroxyl groups were protected with tert-butyldimethylsilyl chloride to give 4 and subsequent selective deprotection of the 5 ' position under mild acidic conditions led to compound $\mathbf{5}$.

The synthesis of the oxetane model $\mathbf{1 0}$ started with the commercial thymine acetic acid as shown in Scheme 5.3. First, the benzyl ester $\mathbf{6}$ was prepared by esterification. Then, it was irradiated in the presence of benzophenone in a Pyrex vessel $\boldsymbol{\lambda}>$ $290 \mathrm{~nm}$ ) with a medium pressure mercury lamp (125W) to give a mixture of thymine oxetanes as the result of a Paternò-Büchi reaction. ${ }^{29}$ Then, the stable oxetane 7 was isolated by flash chromatography. The catalytic hydrogenolysis of the benzyl ester 7 led to the oxetane acetic acid derivative $\mathbf{8}$, and subsequent esterification with $\mathbf{5}$ gave the corresponding model 9. Finally, deprotection of 3'-hydroxyl group under mild conditions gave the oxetane model 10 .

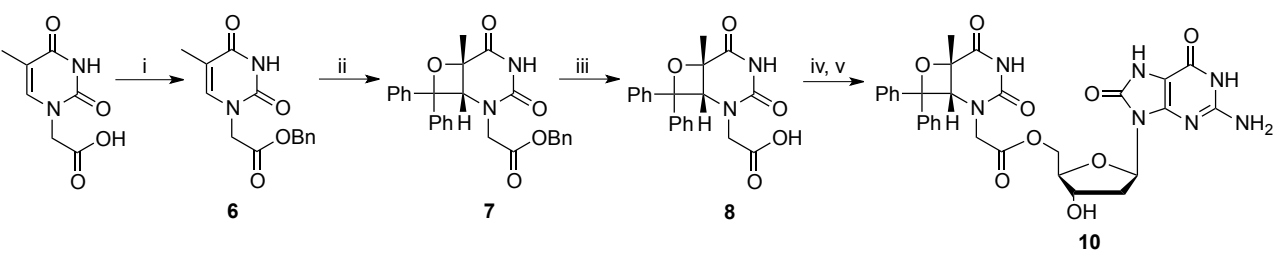

Scheme 5.3. Synthetic strategy to prepare 10. Reagents and conditions: (i) CDI, BnOH, DMF, rt, 18h; (ii) benzophenone, hv, $\lambda>290 \mathrm{~nm}, \mathrm{MeCN}, 16 \mathrm{~h}$; (iii) $10 \% \mathrm{Pd} / \mathrm{C}, \mathrm{H}_{2}, \mathrm{AcOH}, \mathrm{rt}$, 4h; (iv) 5, EDC, TBTU, DMAP, DMF, rt, 20h; (v) TBAF, THF, rt, 16h.

Secondly, we decided to synthesize four models $(15,16,20$ and 21, Figure 5.1) where $O G$ or $G$ nucleobases are covalently attached to a cyclobutane thymine dimer or an oxetane through a flexible and long linker. This linker was shown to favor the splitting efficiency of the four-membered ring in polar solvents through a preferred U- 
shaped conformation, induced by hydrophobic interactions, which shortens the spatial distance between donor and acceptor. ${ }^{31}$ The first step of the synthesis was to obtain the hydroxyethyl derivatives $\mathbf{1 2}$ and $\mathbf{1 4}$ as shown in Scheme 5.4. The compound $\mathbf{1 2}$ was easily synthetized from 2-amino-6-chloropurine in two-steps. First, the linker was introduced through alkylation with 2-bromoethoxy-tert-butyldimethylsilane to afford the N-9 alkylated isomer 11 with a high selectivity, this step was followed by hydrolysis to give the deprotected compound 12 . Subsequent bromination at the C8 position of 12 and hydrolysis with sodium acetate in acetic acid led to compound 14 .

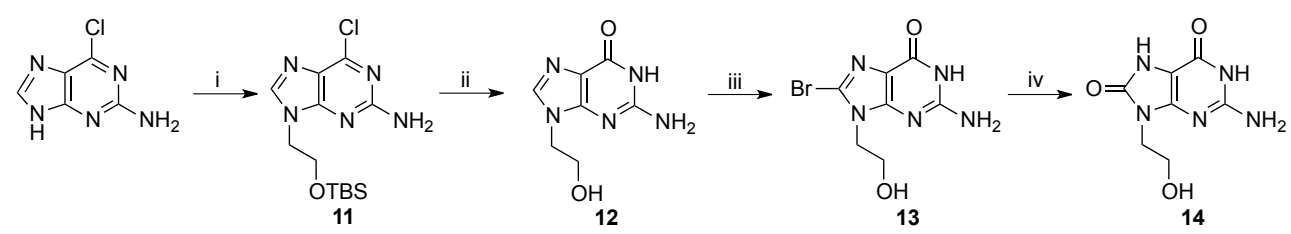

Scheme 5.4. Synthetic strategy to prepare 12 and 14 . Reagents and conditions: (i) 2-bromoethoxy-tert-butyldimethylsilane, $\mathrm{NaH}, \mathrm{DMF}, \mathrm{rt}, 24 \mathrm{~h}$; (ii) $2 \mathrm{M} \mathrm{HCl}$, $100{ }^{\circ} \mathrm{C}, 2 \mathrm{~h}$; (iii) NBS, $\mathrm{MeCN} / \mathrm{H}_{2} \mathrm{O}, \mathrm{rt}, 30 \mathrm{~min}$; (iv) $\mathrm{AcONa}, \mathrm{AcOH}, 130^{\circ} \mathrm{C}, 7 \mathrm{~h}$.

The synthesis of the oxetane models $\mathbf{1 5}$ and $\mathbf{1 6}$ is shown in Scheme 5.5, they were obtained by esterification of the oxetane acetic acid derivative 8 , with 14 and 12 , respectively.

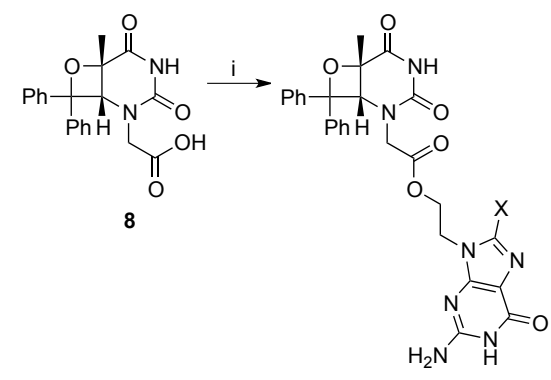

$$
\begin{aligned}
& \text { 15: } \mathrm{X}=\mathrm{OH} \\
& \text { 16: } \mathrm{X}=\mathrm{H}
\end{aligned}
$$

Scheme 5.5. Synthetic strategy to prepare 15 and 16. Reagents and conditions: (i) 12 or 14, EDC, TBTU, DMAP, DMF, rt, 24h. 
For the CPD dyads, the cyclobutane pyrimidine dimer was covalently attached to two chromophores to avoid the formation of a mixture of mono- or disubstituted compounds. The preparation of the cis-syn thymine dimer was performed in four steps from thymine acetic acid as shown in Scheme 5.6. First, the ethyl ester 17 was prepared by Fischer esterification; then it was irradiated in a Pyrex vessel $(\lambda>290$ $\mathrm{nm})$ with a medium pressure mercury lamp (125W) using acetone as photosensitizer to give a mixture of four isomers. The cis-syn thymine dimer $\mathbf{1 8}$ was separated by flash chromatography and its structural assignment was confirmed by ${ }^{1} \mathrm{H}$ NMR, in particular the chemical shift of the proton at N3 at ca. 10.51 ppm was the same as described in the literature. ${ }^{26}$ Hydrolysis yielded the cis-syn thymine dimer acetic acid derivative 19, and subsequent esterification with $\mathbf{1 4}$ and $\mathbf{1 2}$ gave the corresponding 20 and $\mathbf{2 1}$ cyclobutane dimer models, respectively.

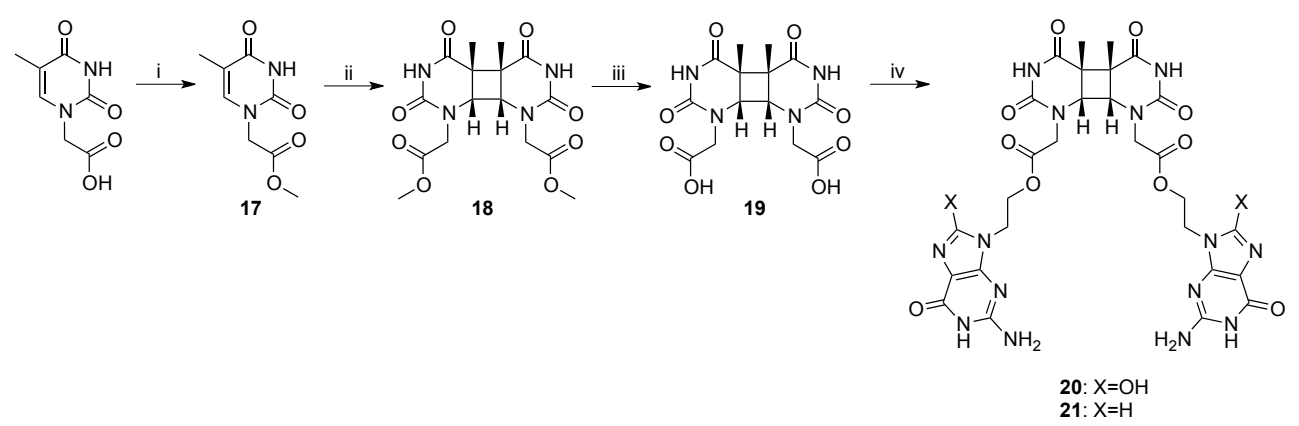

Scheme 5.6. Synthetic strategy to prepare 20 and 21. Reagents and conditions: (i) $\mathrm{H}_{2} \mathrm{SO}_{4}, \mathrm{MeOH}, 100{ }^{\circ} \mathrm{C}, 24 \mathrm{~h}$; (ii) acetone/ $\mathrm{MeCN}, \mathrm{h} v, \lambda>290 \mathrm{~nm}, 72 \mathrm{~h}$; (iii) 5 $\mathrm{M} \mathrm{HCl}, 100^{\circ} \mathrm{C}, 30 \mathrm{~min}$; (iv) 12 or 14, EDC, TBTU, DMAP, DMF, rt, 24h.

\subsubsection{Photocycloreversion}

The UV absorbance spectra of $\mathbf{1 0}$ is shown in Figure 5.2 (left). We can observe the characteristic band of OG chromophore with a maximum at ca. $295 \mathrm{~nm}$. In order to study the splitting of the four-membered ring model, steady-state photolysis 
was performed using a monochromatic excitation at $308 \mathrm{~nm}$ where the absorption of the oxetane unit (Figure 5.2, right) is negligible, and thus the photons are mainly absorbed by the OG chromophore.
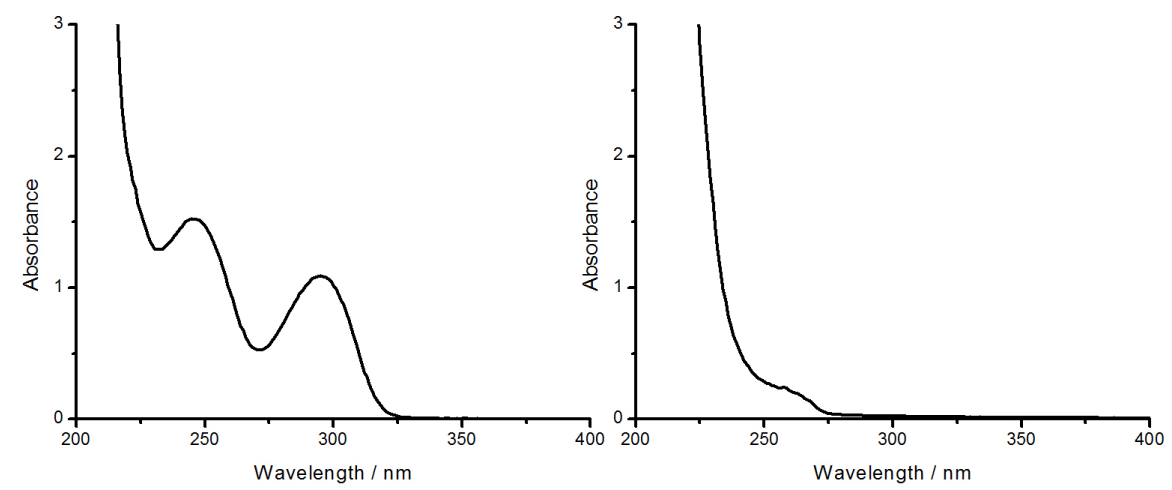

Figure 5.2. UV spectra of $\mathbf{1 0}(0.2 \mathrm{mM})$ in neutral PBS (left) and $\mathbf{7}(0.2 \mathrm{mM})$ in $\mathrm{MeCN}$ (right).

Firstly, the photosplitting of the model 10 at $308 \mathrm{~nm}$ was followed by UV spectrophotometry, monitoring the absorption bands at $270 \mathrm{~nm}$ and $250 \mathrm{~nm}$ that inform on the generation of the thymine and benzophenone (BP) chromophores, respectively, as shown in Scheme 5.7.

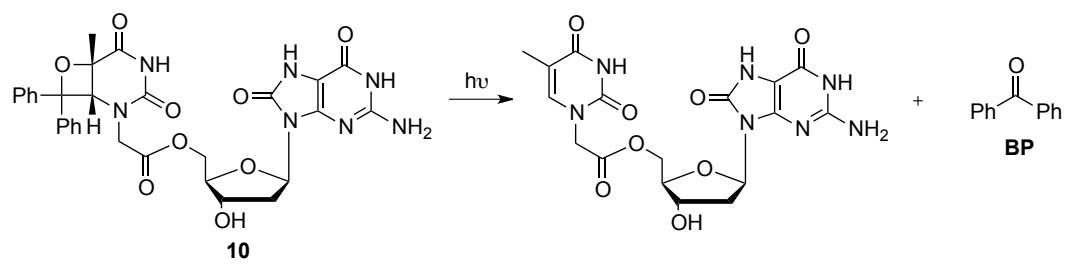

Scheme 5.7. Cleavage reaction of 10 .

As shown in Figure 5.3 left, an increase of the absorption at $270 \mathrm{~nm}$ and 250 $\mathrm{nm}$ was observed during the irradiation at $308 \mathrm{~nm}$, which agrees with the formation of thymine chromophore attached to OG and BP. In order to ensure that the photo- 
cleavage resulted in the expected photoproducts, the course of the reaction was monitored by HPLC at different irradiations times. Figure 5.3 right shows the cleavage of the oxetane model 10, that eluted at $5.7 \mathrm{~min}$, into thymine photoproduct, with retention time of $4.14 \mathrm{~min}$, and BP, with retention time of $14.1 \mathrm{~min}$. This assignment was confirmed by HPLC co-elution with standard compounds.
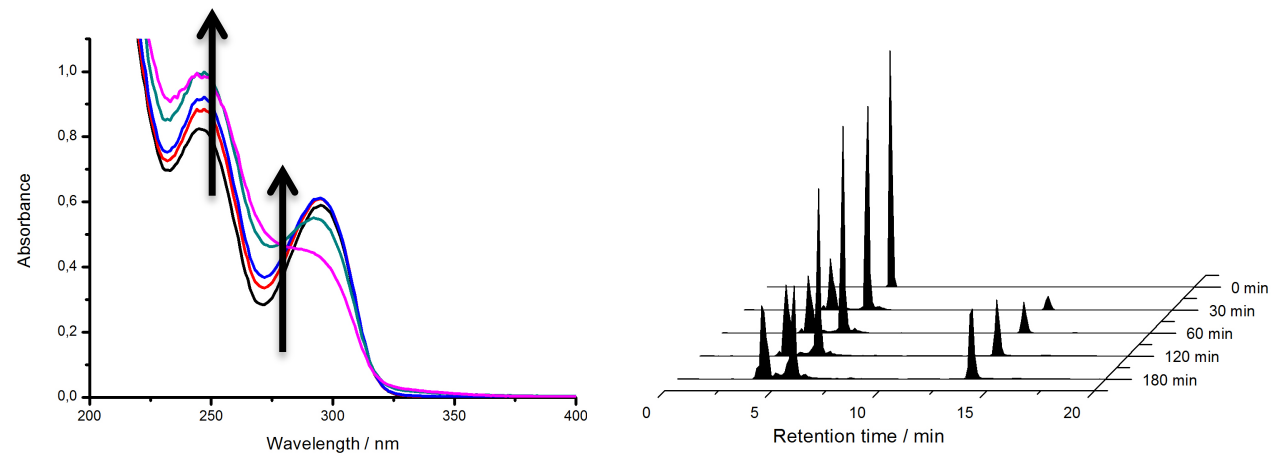

Figure 5.3. Cleavage reaction of $\mathbf{1 0}$ monitored by UV spectroscopy (left) and HPLC (right) at 0, 30, 60, 120 and 180 min of irradiation at $308 \mathrm{~nm}$ in neutral PBS.

Therefore, these data show that the photocycloreversion of oxetane model 10 involves the excited state of OG, which donates an electron to split the four-membered ring. Fluorescence experiments can support this PET mechanism from singlet excited state of PS. However, given the ultralow fluorescence quantum yields of OG (1.3 x10$\left.{ }^{4}\right)^{20}$, it was not possible to observe this intramolecular fluorescence quenching as an evidence of the electron transfer process. However, this ultrafast process can be investigated by means of femtosecond transient absorption spectroscopy, following the evolution of singlet-singlet transient absorption band (from ${ }^{1} \pi \pi^{*}$ ) of OG but also intermediate species such as radical cation or anion. In this context, preliminary femtosecond transient absorption studies with 10 and OG nucleoside were performed in Carlos Crespo's group during my first predoctoral stay. Therefore, if the electron 
transfer takes place from $O G$, the radical cation of $O G$ and radical anion of BP should be detected. In this way, back to back experiments (same aborption for both compounds) with $308 \mathrm{~nm}$ excitation were performed for OG and 10 in neutral PBS as shown in Figure 5.4. As seen for both compounds, a transient species grows in from 0.0 to $0.4 \mathrm{ps}$ at $570 \mathrm{~nm}$ along with another band shoulder around $350 \mathrm{~nm}$. In the subsequent time region from 0.4 to $4 \mathrm{ps}$, the absorption bands decrease. The spectra do not show changes after 4 ps, indicating that the excited molecules had come back to the ground state.

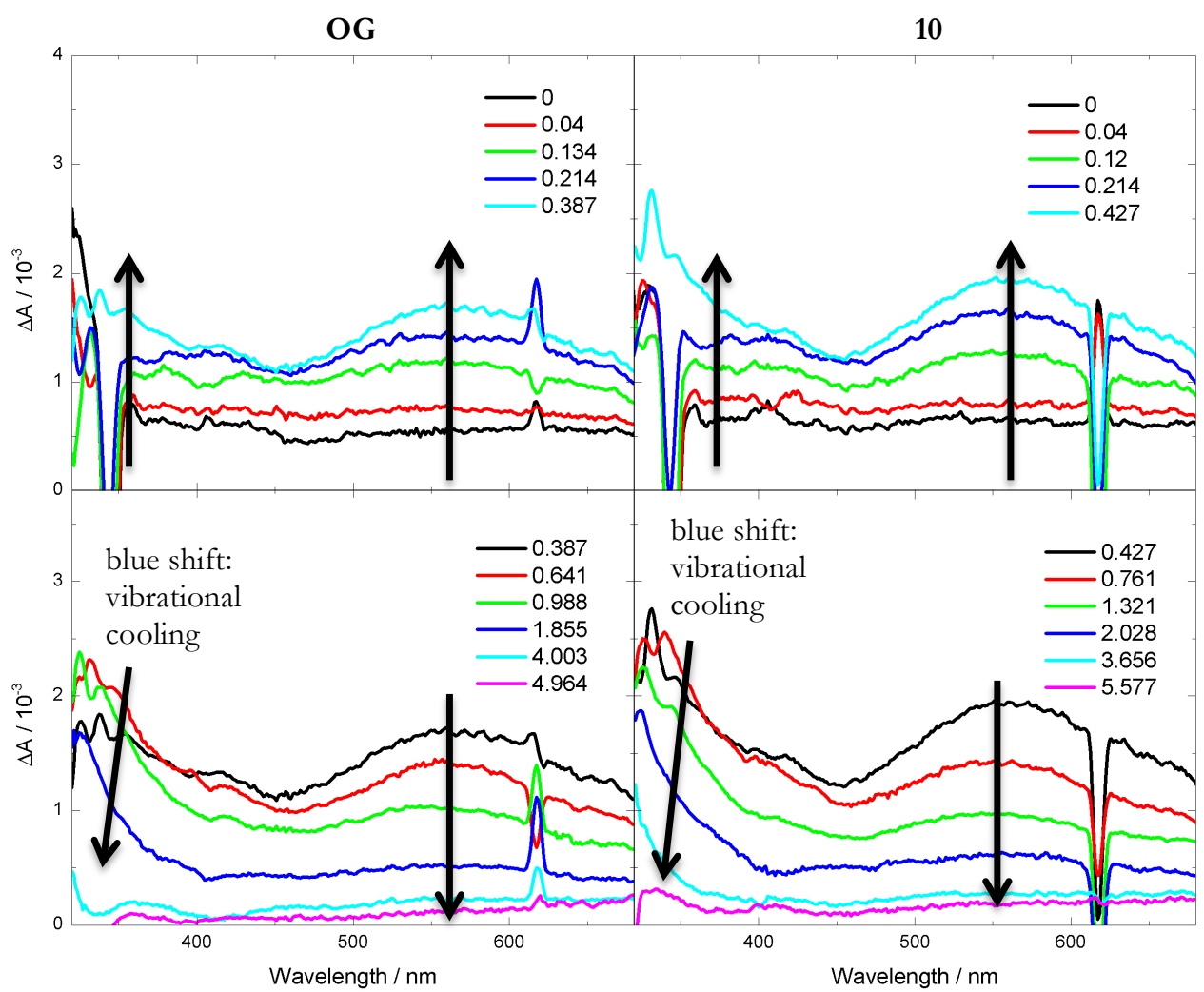

Figure 5.4. Transient absorbance changes of OG (OD 0.26) and $\mathbf{1 0}(\mathrm{OD} 0.31)$ in neutral PBS. Overtone at $616 \mathrm{~nm}(2 \times 308 \mathrm{~nm})$. 
In order to examine the excited state dynamics, we performed a global fitting analysis with a sequential kinetic model convoluted with an instrument response time of $200 \pm 50$ fs and coherence spike function. Figure 5.5 shows the best fits at 354 and $568 \mathrm{~nm}$. Two lifetimes of $280 \mathrm{fs}$ and $1.51 \mathrm{ps}$ for $\mathrm{OG}$ and $240 \mathrm{fs}$ and $1.29 \mathrm{ps}$ for 10, were determined. Therefore, we have assigned the $570 \mathrm{~nm}$ transient species to the ${ }^{1} \pi \pi^{*}$ electronic transition of OG neutral form and the initial growth of the $350 \mathrm{~nm}$ signal to vibrational cooling of the hot ground-state of OG neutral form, as already described for other nucleosides.

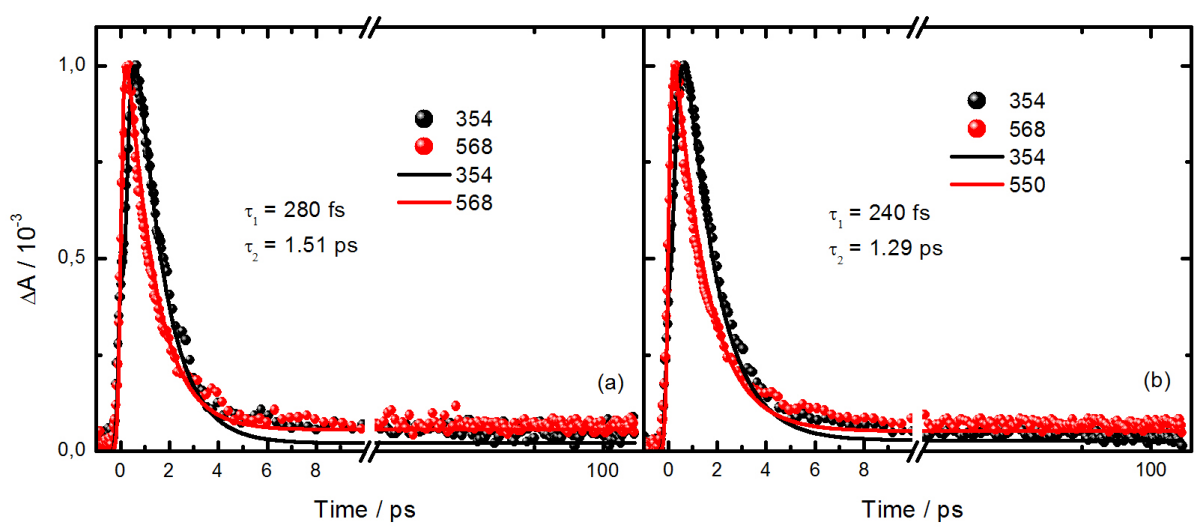

Figure 5.5. Kinetic traces and fits obtained from the global fitting analysis for: (a) OG and (b) 10 in neutral PBS.

Comparison of the spectra and kinetics of both compounds show the same excited species. It indicates that the transient species of $\mathbf{1 0}$ proceed only from excited state of OG. These results suggest that the molecules are unstacked and not close enough to allow efficient electron transfer. In this way, we carried out the experiments of transient absorption for OG and $\mathbf{1 0}$ in ethylene glycol, which is more viscous than PBS and might favor the stacked conformation. The same transient species were observed, and the only difference relies on an increase of the lifetimes. Unfortunately, 
comparison of the data obtained with OG and $\mathbf{1 0}$ did not allow to draw clear conclusions.

In summary, since the oxetane has no significant absorption at $308 \mathrm{~nm}$, OG absorbs a photon producing the excited state ${ }^{1} \mathrm{OG}^{*}$-OXT (Scheme 5.8). Then the excited state may relax as follows: fluorescence $\left(k_{\mathrm{f}}\right)$, internal conversion $\left(\mathrm{k}_{\mathrm{ic}}\right)$, and electron transfer to the linked oxetane $\left(k_{\mathrm{et}}\right)$. The charge-separated species formed via electron transfer can undergo two processes, oxetane splitting $\left(\mathrm{k}_{\text {spl }}\right)$ or back electron transfer (kbet). Therefore, there are two different competitions in these processes: (1) the photophysical process $\left(\mathrm{k}_{\mathrm{f}}\right.$ and $\left.\mathrm{k}_{\mathrm{ic}}\right)$ with electron transfer $\left(\mathrm{k}_{\mathrm{et}}\right)$ and $(2)$ the splitting $\left(\mathrm{k}_{\mathrm{spl}}\right)$ with back electron transfer ( $\left.k_{\mathrm{bet}}\right)$.

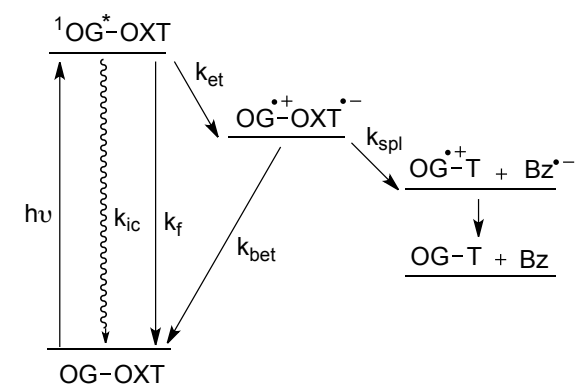

Scheme 5.8. Photophysical and photochemical processes of model 10 where OG is attached to an oxetane (OXT).

In model systems, back electron transfer $\left(\mathrm{k}_{\mathrm{bet}}\right)$ was reported to lead to low splitting efficiencies. ${ }^{27-28}$ However, internal conversion $\left(k_{i c}\right)$ also plays an important role for PS with short lived excited states, as observed for flavin model systems..$^{32}$ Therefore, in the case of 10 , as the fluorescence process $\left(\mathrm{k}_{\mathrm{f}}\right)$ can be neglected for OG, the main process competing with electron transfer is the nonradiative process $\left(k_{i c}\right)$. During our measurements, the observed species for $\mathbf{1 0}$ proceed only from $\left({ }^{1} \pi \pi^{*}\right)$ excited state of OG in both solvents, PBS and ethylene glycol. This might be due to the fact that $\mathbf{1 0}$ is almost unstacked, and radical ion pairs are not formed in a detectable quantity, the 
Chapter 5: Photorepair of Thymine Derived Cyclobutanes and Oxetanes by 8-oxoGuanine

electron transfer process being too inefficient to compete with the fast nonradioactive deactivation of OG.

In addition, the absorption bands of the formed radicals can be overlapped in the spectra with OG absorption bands at 350 and $570 \mathrm{~nm}$. It was reported that the radical cation of OG has a narrow absorption band with maximum at $320 \mathrm{~nm}$ and a shoulder near $400 \mathrm{~nm}$, which decays on millisecond time scales. ${ }^{33}$ Concerning the radical anion of $\mathrm{BP}$, formed after cleaving the radical anion of oxetane, it has a maximum band at $680 \mathrm{~nm} .{ }^{34}$ However, the latter species is in the limit of our detection window and, thus, it cannot be detected under our experimental conditions. Altogether, these data made us redefine the model in order to use a long and flexible linker, to bring closer the donor and acceptor moieties. The selected linker has been used in previous femtosecond spectroscopy of a thymine dimer model attached to a flavin or indole chromophore. ${ }^{32,35}$ Therefore, we synthetized four models where OG and G nucleobases are covalently linked to an oxetane unit (15 and 16, Figure 5.1) and to a cyclobutane thymine dimer (20 and $\mathbf{2 1}$, Figure 5.1) by a 5-chain flexible linker, in order to compare the ability of $O G$ and $G$ as electron donors.

The UV absorption spectra of 15, 16, 20 and 21 are shown in Figure 5.6a. In case of 15 and 20 , we can observe the characteristic band of OG chromophore with a maximum at ca. $295 \mathrm{~nm}$, and that exhibits a red shifted absorption by respect to the guanine derivatives $\mathbf{1 6}$ and $\mathbf{2 1}$. In order to study the splitting of the four-membered ring models, steady-state photolysis was performed using a monochromatic excitation at $280 \mathrm{~nm}$. We selected this wavelength because it corresponds to the point where the four compounds show the same absorbance and, thus, the splitting can be achieved, for the same irradiation time, by the same number of absorbed photons for the four dyads. In addition, at this wavelength and under the conditions of concentration used (of ca. $10^{-4} \mathrm{M}$ ) the absorption of the CPD and oxetane unit is very small as shown in 
Figure 5.6b., and thus the photons are mainly absorbed by the chromophores OG and G.
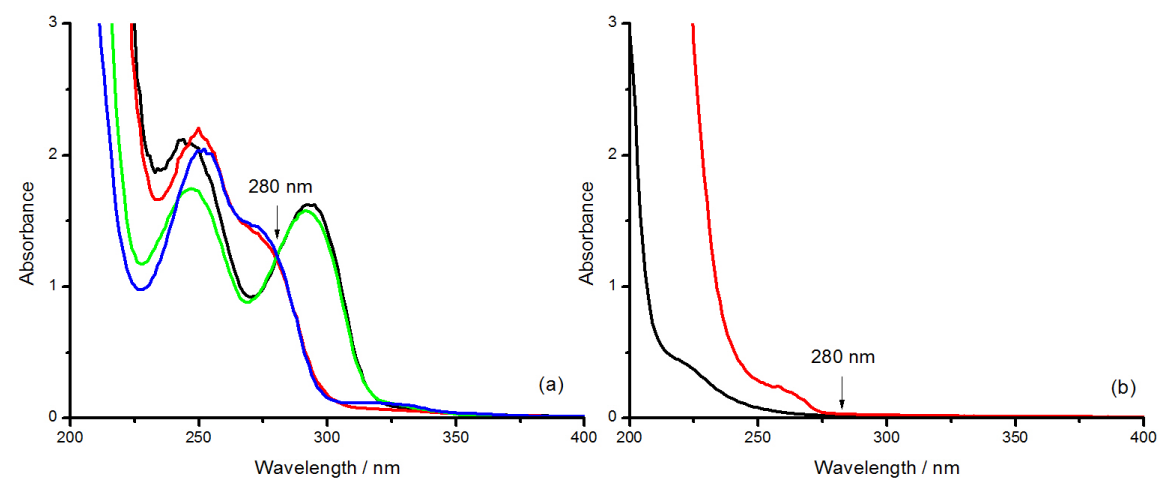

Figure 5.6. UV absorption spectra of: (a) $15(0.2 \mathrm{mM}$, black), $16(0.2 \mathrm{mM}$, red), 20 (0.1 mM, green) and 21 (0.1 mM, blue) in PBS at pH 7.4; (b) $18(0.1 \mathrm{mM}$, black) and 7 (0.2 mM, red) in MeCN.

Firstly, the photosplitting of the models 15, 16, 20 and 21 at $280 \mathrm{~nm}$ was followed by UV spectrophotometry, monitoring the absorption band at $270 \mathrm{~nm}$ that informs on the generation of the thymine chromophore present in the purported photoproducts 22 and 23 (Scheme 5.9). 


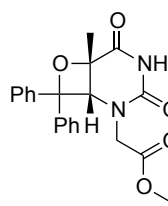<smiles></smiles>

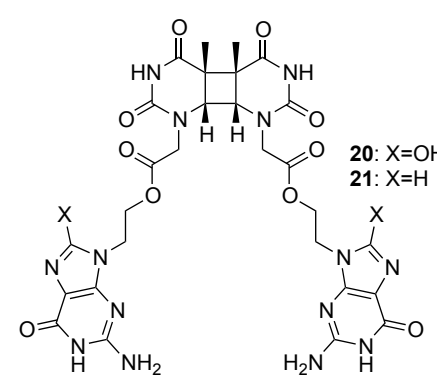

Scheme 5.9. Cleavage reaction of 15, 16, 20, 21 to form 22 or 23 and BP (in the case of $\mathbf{1 5}$ and $\mathbf{1 6}$ ).

As shown in Figure 5.7, an increase of the absorption at $270 \mathrm{~nm}$ was observed during the irradiation of all the compounds. The changes are, however, more pronounced in the case of the OG derived dyads pointing toward a higher efficiency of the process when the oxidized guanine acts as photoreductant. Moreover, another band was observed at around $250 \mathrm{~nm}$ for oxetane models 15 and 16, which agrees with the formation of BP as a result of the cycloreversion. 


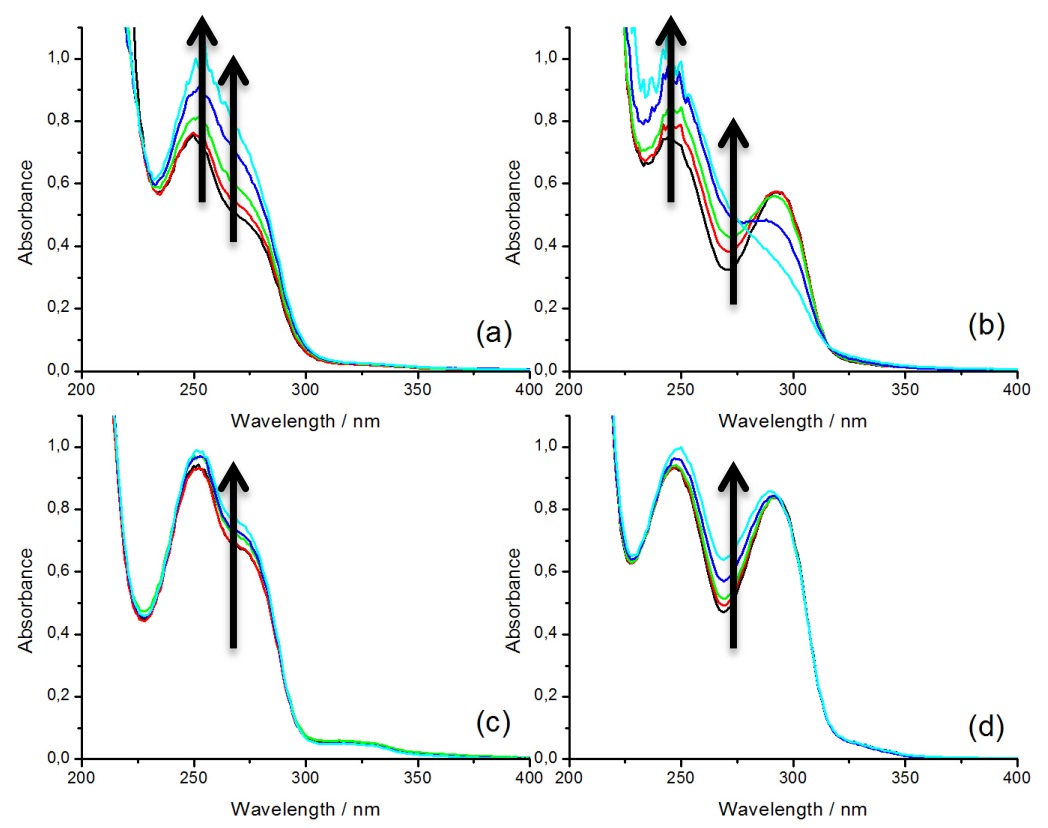

Figure 5.7. UV absorption spectra of: (a) 16 (0.2 mM), (b) $15(0.2 \mathrm{mM})$, (c) 21 $(0.1 \mathrm{mM})$, (d) $20(0.1 \mathrm{mM})$ in PBS at $\mathrm{pH} 7.4$ obtained after 0, 30, 60, 120 and $180 \mathrm{~min}$ of irradiation at $280 \mathrm{~nm}$.

In addition, the cleavage was achieved at $295 \mathrm{~nm}$ to check that the observed cycloreversion is not the result of direct absorption by the oxetane and CPD unit. As shown in Figure 5.8 similar results were obtained for the two irradiation conditions, i.e. at 280 and $295 \mathrm{~nm}$. 


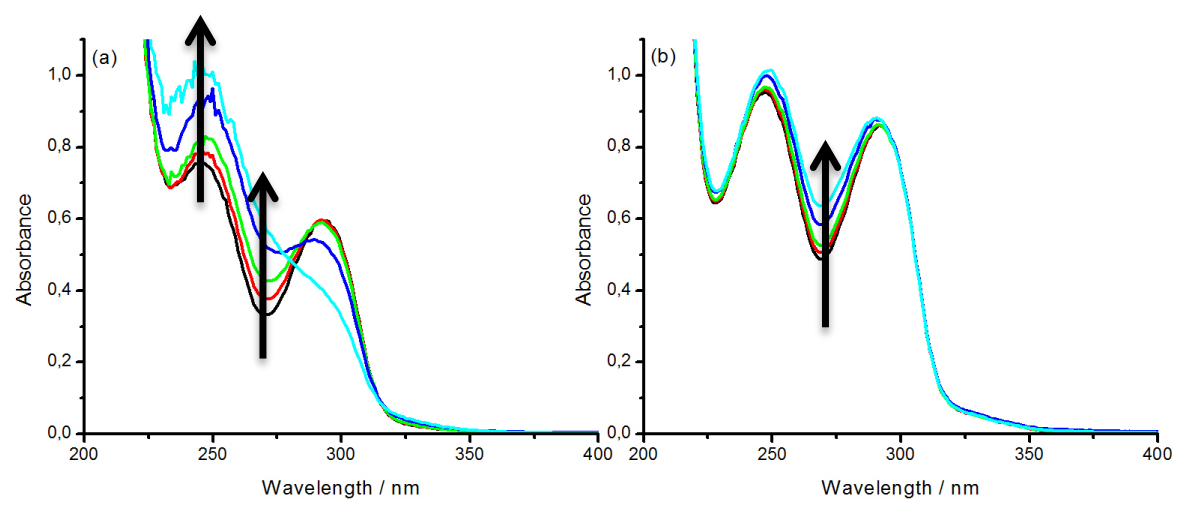

Figure 5.8. UV spectra of: (a) $15(0.2 \mathrm{mM})$, (b) $20(0.1 \mathrm{mM})$ in PBS at pH 7.4 obtained after 0, 30, 60, 120 and 180 min of irradiation at $295 \mathrm{~nm}$.

In order to ensure that the photocleavage resulted in the expected photoproducts, the course of the reaction was monitored by HPLC at different irradiations times. Figure 5.9 shows the cleavage of the oxetane models 15 and $\mathbf{1 6}$, that eluted at 30.8 min, into 22 or $\mathbf{2 3}$, with retention time of $19.4 \mathrm{~min}$, and BP, with retention time of $38.4 \mathrm{~min}$.
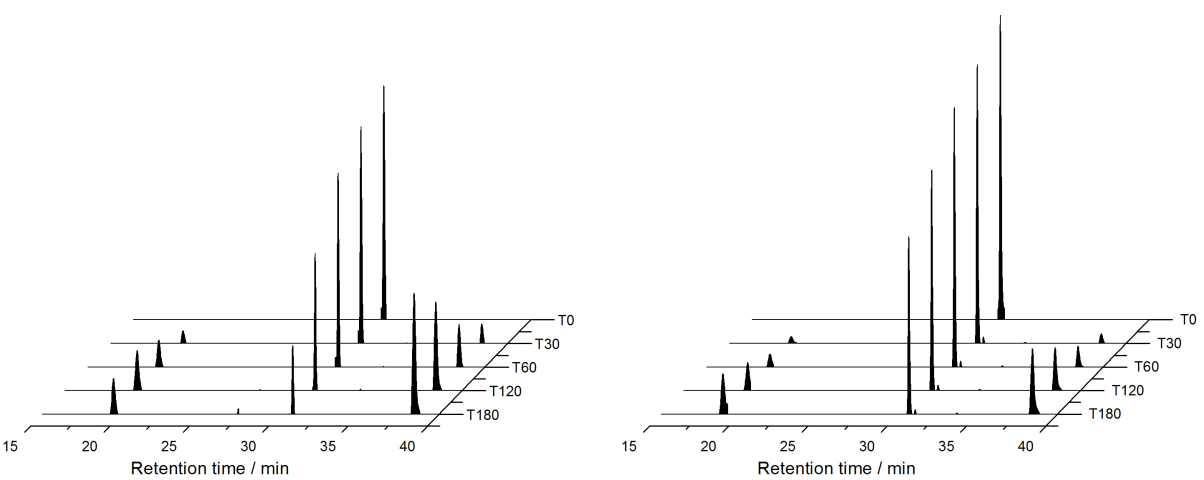

Figure 5.9. HPLC chromatograms obtained after 0, 30, 60, 120, 180 min of irradiation of 15 (0.2 mM, left) and 16 (0.2 mM, right) in PBS at pH 7.4 with $280 \mathrm{~nm}$ light. 
For the CPD case, Figure 5.10. shows the photochemical reaction of models 20 and 21, with retention time of $26.1 \mathrm{~min}$ and $23.1 \mathrm{~min}$, respectively, to give the photosplitted product $\mathbf{2 2}$ or $\mathbf{2 3}$ with retention time of $18.5 \mathrm{~min}$. A clean conversion was observed, as no other products were detectable in all cases.
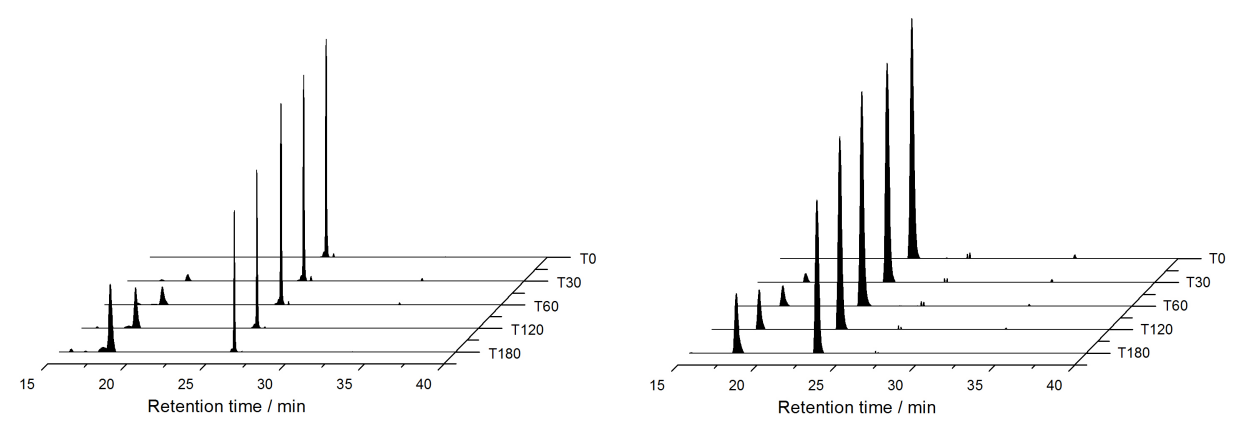

Figure 5.10. HPLC chromatograms obtained after 0, 30, 60, 120, 180 min of irradiation of $20(0.1 \mathrm{mM}$, left $)$ and $21(0.1 \mathrm{mM}$, right $)$ in PBS at $\mathrm{pH} 7.4$ with $280 \mathrm{~nm}$ light.

To determine the efficiency of the oxetane and CPD models, we measure the splitting quantum yield, $\phi=$ (rate of formation of repaired model)/(rate of photon absorbed). ${ }^{28}$ Solutions $(3 \mathrm{~mL}$ ) of 15, 16, 20 and 21 were irradiated at $280 \mathrm{~nm}$ and the increase in absorbance at $270 \mathrm{~nm}$ was monitored due to the regeneration of the 5,6double bond of pyrimidine 22 or 23 and BP $\left(\varepsilon_{\text {thymine }}=7500 \mathrm{~L} \mathrm{~mol}^{-1} \mathrm{~cm}^{-1}\right.$ and $\varepsilon_{\text {thy- }}$ ${ }_{\text {mine }+\mathrm{BP}}=19400 \mathrm{~L} \mathrm{~mol}^{-1} \mathrm{~cm}^{-1}$ determined by UV spectroscopy). The splitting concentration $\left(\mathrm{c}_{\mathrm{sp}}\right)$ was obtained through $\Delta \mathrm{A}_{270} / \Delta \varepsilon_{270}$ as stated by the Beer-Lambert equation. Therefore, the plot of $\mathrm{c}_{\mathrm{spl}}$ against the irradiation time gives a well-fitted straight line whose slope (B), which is a splitting rate of the model compounds. Moreover, to reduce competition in absorbing the incident light at $280 \mathrm{~nm}$ between the models and the photoproducts, the splitting extent was controlled within $5 \%$ in all measurements. 
Regarding to the number of photons absorbed by the models, the intensity of the light beam was measured by ferrioxalate actinometry, being $\mathrm{I}_{0}=2.29 \times 10^{-8}$ einstein $\min ^{-1}{ }^{36}$ Therefore, the rate of photons absorbed by models $\left(\mathrm{I}_{\mathrm{A}}\right)$ was obtained from the absorbance at $280 \mathrm{~nm}$ (irradiation wavelength) before irradiation, in terms of Beer's law (equation 5.1).

$$
I_{A}=I_{0}\left(1-10^{A^{280}}\right)
$$

These values allowed the determination of the quantum yield $\Phi$ through equation 5.2 where $V_{0}$ is the volume of irradiation solution.

$$
\phi=\frac{\text { rate of formation of repaired model }}{\text { rate of photon absorbed }}=\frac{B}{I_{A}} V_{O}
$$

The observed quantum yields for 15, 16, 20 and 21 were calculated and listed in Table 5.1. A more efficient splitting was observed for oxetane than for CPD models. In addition, different quantum yields were determined when $O G$ and $G$ are acting as the photoreductants; the photocycloreversion being almost twice as efficient in the case of the former. These values are in agreement with previous studies that have shown that the cleavage quantum yield is often much less than unity due to efficient back electron transfer when a thymine dimer or oxetane is covalently tethered to an electron-donating chromophore such as indole ${ }^{28-29}$ or flavin ${ }^{27}$. Therefore, all photoinduced electron transfer-initiated chemical reactions compete with an exothermic back electron-transfer process, and quantum yields approaching the unity are reached when the rate of the chemical process is fast relative to the rate of back electron transfer.

Moreover, the obtained results are in agreement with the quantum yields reported for models where oxetane and CPD are covalently linked to flavin, with a value two fold higher for the heterocycle than for the cyclobutane ring splitting (ca. of 0.02 and 0.01 , respectively). ${ }^{27}$ Indeed, this difference can be explained by comparing the splitting rate of the oxetane radical anion $\left(>10^{7} \mathrm{~s}^{-1}\right)^{34}$, which is faster than that of the 
CPD radical anion $\left(\sim 10^{6} \mathrm{~s}^{-1}\right)^{37}$. Thus, the fast splitting of the oxetane radical anion competes more efficiently with BET within the zwitterionic intermediate than the radical anion of CPD does. Interestingly in photolyases, the higher efficiency of CPD photolyase (ca. of 0.7-0.9) by contrast with (6-4) photolyase (ca. of 0.1 ) has been attributed to factors that suppress back electron transfer. ${ }^{38}$

Table 5.1. Splitting efficiencies for compounds 15, 16, 20 and 21 in neutral PBS.

\begin{tabular}{ccccc}
\hline & 15 & 16 & 20 & $\mathbf{2 1}$ \\
\hline$\phi$ & 0.08 & 0.04 & 0.02 & 0.01
\end{tabular}

Regarding to the chromophore, the splitting is more efficient for 15 and $\mathbf{2 0}$ models, that is, when excited OG is the electron donor. The efficiency and ability of these photosensitizers to induce a reductive dimer splitting is related to their excited-state oxidation potentials $\mathrm{E}_{\mathrm{ox}}^{\mathrm{D}^{*}}$, which are listed in Table 5.2. They were determined from literature values from the oxidation potential $\left(\mathrm{E}_{\mathrm{ox}}^{\mathrm{D}}\right)^{11}$ and the singlet state energy, $\mathrm{E}^{*}$ (using 310 and $300 \mathrm{~nm}$ absorption tail as the 0-0 transition energy for $O G$ and $G$, respectively), using the equation 5.3.

$$
E_{o x}^{D *}=E_{o x}^{D}-\frac{E^{*}}{23.06}
$$

Interestingly, the $\mathrm{OG}$ has a more negative $\mathrm{E}_{\mathrm{ox}}^{\mathrm{D}^{*}}$ than $\mathrm{G}$ or even than $\mathrm{FADH}^{-}$, which agrees with its higher efficiency for cycloreversion. In order to know if the PET from $G$ and $O G$ to oxetane or CPD is an energetically favorable process, the free energy associated with the PET $\left(\Delta \mathrm{G}_{\mathrm{ct}}\right)$ was determined according to the Rehm-Weller equation 5.4 and using the previously reported $\mathrm{E}_{\text {red }}^{\mathrm{A}}$ of ca. -2.2 V vs. NHE for both thymine dimer ${ }^{39}$ and oxetane $e^{40}$.

$$
\Delta G_{c t}=23.06\left(E_{o x}^{D}-E_{r e d}^{A}\right)-E^{*}
$$


As shown in Table 5.2, the process is more exergonic as $\mathrm{E}_{\mathrm{ox}}^{\mathrm{D}^{*}}$ became increasingly negative, being most favorable for OG. These results agree with a PET mechanism from the singlet excited state of $O G$ and $G$ to the CPD and oxetane units.

Table 5.2. Photophysical properties of the selected PS.

\begin{tabular}{ccccc}
\hline PS & $\begin{array}{c}\mathbf{E}_{\mathbf{o x}}^{\mathbf{D}} / \mathbf{V} \text { vs. } \\
\mathbf{N H E}\end{array}$ & $\begin{array}{c}\mathbf{E}_{\mathbf{0 x}}^{\mathbf{D}^{*}} / \mathbf{V} \\
\mathbf{v s .} \mathbf{N H E}\end{array}$ & $\mathbf{E}^{*} / \mathbf{k c a l ~ m o l}^{-1}$ & $\begin{array}{c}\Delta \mathbf{G} / \mathbf{k c a l} \\
\mathbf{m o l}^{-1}\end{array}$ \\
\hline OG & 0.711 & -3.3 & 92.2 & -25.3 \\
$\mathrm{G}$ & $1.2^{11}$ & -2.9 & 95.3 & -16.9 \\
$\mathbf{F A D H}^{-}$ & $\mathbf{0 . 1 2 ^ { 4 1 }}$ & $\mathbf{- 2 . 8 4 1}$ & $\mathbf{6 3 . 6 4 1}$ & -10.1 \\
\hline
\end{tabular}

As mentioned above, fluorescence experiments can support the PET mechanism from singlet excited state of PS. However, given the ultralow fluorescence quantum yields of $\mathrm{G}$ and $\mathrm{OG}\left(2.3 \text { and } 1.3 \times 10^{-4} \text {, respectively }\right)^{20}$, it was not possible to observe the intramolecular fluorescence quenching as an evidence of the electron transfer process. In addition, up conversion fluorescence might be a useful technique to study the PET between OG and the four-membered ring in $\mathbf{1 5}$ and $\mathbf{2 0}$ models, because it could provide a cleaner decay of ${ }^{1} \mathrm{OG}^{*}$ without potential interference of others species such as radical anion or cation. Therefore, fluorescence experiments at the femtosecond timescale are in progress in collaboration with Thomas Gustavsson's group.

\subsection{Conclusions}

We have synthetized model systems of thymine -derived oxetane and cyclobutane dimers connected to guanine or 8-oxoguanine, which act as photoreductants. The photocleavage of the four-membered rings was followed by UV spectrophotometry and HPLC. A more efficient cleavage was observed for OG than for G, which is related with the more negative oxidation potential of the former in its excited state. 
Moreover, $G$ is able to split the models with similar efficiency than the flavin-systems. Therefore the electron donor ability has been proven for $G$ and for $O G$, with similar or even higher efficiency than $\mathrm{FADH}^{-}$. However, the low quantum yields are the result of competition between unproductive back-electron transfer reaction and the cleavage of the dimer radical anion. In addition, up conversion fluorescence spectroscopy is being run to get more information on the PET mechanism.

\subsection{Experimental section}

\subsubsection{Synthesis}

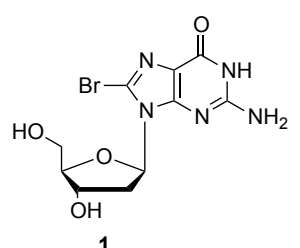

subsequently evaporated to dryness. The residual solid was taken up in $350 \mathrm{~mL}$ acetone and stirred for $4 \mathrm{~h}$ at rt. Subsequently, the mixture was stored at $-20^{\circ} \mathrm{C}$ for $48 \mathrm{~h}$ and filtrated. The solid was washed with cold acetone and dried to yield 1 (11.2 g, 87 $\%$ ) as an orange solid. ${ }^{1} \mathbf{H}$ NMR (300 MHz, DMSO) $\delta 10.79$ (s, 1H), 6.49 (s, 2H), 6.15 $(\mathrm{t}, J=7.3 \mathrm{~Hz}, 1 \mathrm{H}), 5.25(\mathrm{~d}, J=3.8 \mathrm{~Hz}, 1 \mathrm{H}), 4.85(\mathrm{~s}, 1 \mathrm{H}), 4.39$ (d, $J=2.8 \mathrm{~Hz}, 1 \mathrm{H})$, $3.79(\mathrm{dd}, J=8.4,5.4 \mathrm{~Hz}, 1 \mathrm{H}), 3.62(\mathrm{~d}, J=10.8 \mathrm{~Hz}, 1 \mathrm{H}), 3.50(\mathrm{~d}, J=5.5 \mathrm{~Hz}, 1 \mathrm{H}), 3.26$ - $3.06(\mathrm{~m}, 1 \mathrm{H}), 2.10(\mathrm{~m}, 1 \mathrm{H}) .{ }^{13} \mathrm{C}$ NMR (75 MHz, DMSO) $\delta 155.5$ (C), $153.30(\mathrm{C})$, $151.96(\mathrm{C}), 120.5(\mathrm{C}), 117.4(\mathrm{C}), 87.9(\mathrm{CH}), 85.0(\mathrm{CH}), 70.8(\mathrm{CH}), 62.4\left(\mathrm{CH}_{2}\right), 36.5$ $\left(\mathrm{CH}_{2}\right)$.

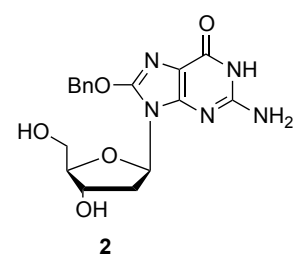

8-benzyloxy-2'-dG (2). Freshly cut Na metal (3.3 g, 144.9 mmol) was dissolved in benzyl alcohol $(100 \mathrm{~mL})$ at $60{ }^{\circ} \mathrm{C}$ until the solution was homogeneous under nitrogen. To the resulting mixture was added $1(10.0 \mathrm{~g}, 28.9 \mathrm{mmol})$ in DMSO $(40 \mathrm{~mL})$, and the mixture was heated at $65{ }^{\circ} \mathrm{C}$ for $24 \mathrm{~h}$. Then cooled to room 
Chapter 5: Photorepair of Thymine Derived Cyclobutanes and Oxetanes by 8-oxoGuanine

temperature, the reaction was poured into magnetically stirred ether $(1000 \mathrm{~mL})$ solvent for precipitation. The precipitated solid was filtered and precipitated with ether one more time. Then was poured in a $3 \mathrm{~mL}$ of water overnight to give a white precipitated $2(3.0 \mathrm{~g}, 30 \%)$. ${ }^{1} \mathbf{H}$ NMR (300 MHz, DMSO) $\delta 10.56(\mathrm{~s}, 1 \mathrm{H}), 7.42(\mathrm{dt}, J=14.9$, $7.0 \mathrm{~Hz}, 5 \mathrm{H}), 6.31(\mathrm{~s}, 2 \mathrm{H}), 6.08(\mathrm{t}, J=7.1 \mathrm{~Hz}, 1 \mathrm{H}), 5.40(\mathrm{~s}, 2 \mathrm{H}), 5.16(\mathrm{~d}, J=4.0 \mathrm{~Hz}$, $1 \mathrm{H}), 4.77(\mathrm{t}, J=5.7 \mathrm{~Hz}, 1 \mathrm{H}), 4.24(\mathrm{~s}, 1 \mathrm{H}), 3.71(\mathrm{~s}, 1 \mathrm{H}), 3.56-3.37(\mathrm{~m}, 2 \mathrm{H}), 3.02-$ $2.75(\mathrm{~m}, 1 \mathrm{H}), 2.01(\mathrm{~d}, J=3.5 \mathrm{~Hz}, 1 \mathrm{H}) .{ }^{13} \mathrm{C}$ NMR (75 MHz, DMSO) $\delta 155.7$ (C), 152.8 (C), 150.9 (C), 149.9 (C), 135.7 (C), 128.4 (3CH), 110.5 (C), $87.3(\mathrm{CH}), 81.5$ $(\mathrm{CH}), 70.9(\mathrm{CH}), 70.6\left(\mathrm{CH}_{2}\right), 62.1\left(\mathrm{CH}_{2}\right), 36.1\left(\mathrm{CH}_{2}\right)$.

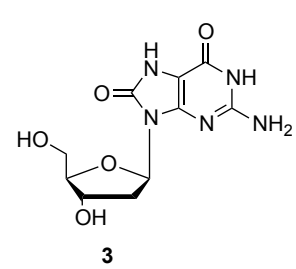

8-oxo-2'-dG (3). To a stirred solution of $2(3.0 \mathrm{~g}, 8.0 \mathrm{mmol})$ in $\mathrm{MeOH}(300 \mathrm{~mL})$ at $\mathrm{rt}$ was added $1.0 \mathrm{M} \mathrm{HCl}(30 \mathrm{~mL})$. The reaction was stirred for $1 \mathrm{~h}$. Removal of water and $\mathrm{MeOH}$ under reduced pressure followed by trituration of the residue from $\mathrm{MeOH} /$ ether afforded quantitative yield of 3. ${ }^{1} \mathbf{H}$ NMR (300 MHz, DMSO) $\delta 6.55(\mathrm{~s}, 2 \mathrm{H}), 6.03(\mathrm{dd}, J=8.1,6.7 \mathrm{~Hz}, 1 \mathrm{H}), 4.32(\mathrm{dd}, J=11.0,8.2$ $\mathrm{Hz}, 1 \mathrm{H}), 3.75$ (d, $J=2.3 \mathrm{~Hz}, 1 \mathrm{H}), 3.57(\mathrm{dd}, J=11.7,4.7 \mathrm{~Hz}, 1 \mathrm{H}), 3.44(\mathrm{dd}, J=11.7$, $4.8 \mathrm{~Hz}, 1 \mathrm{H}), 2.95$ (m, 1H), 1.91 (m, 1H). ${ }^{13} \mathbf{C}$ NMR (75 MHz, DMSO) $\delta 153.8(\mathrm{C})$, $152.2(\mathrm{C}), 151.6(\mathrm{C}), 147.1(\mathrm{C}), 98.6(\mathrm{C}), 87.3(\mathrm{CH}), 80.9(\mathrm{CH}), 71.4(\mathrm{CH}), 62.4\left(\mathrm{CH}_{2}\right)$, $35.5\left(\mathrm{CH}_{2}\right)$.

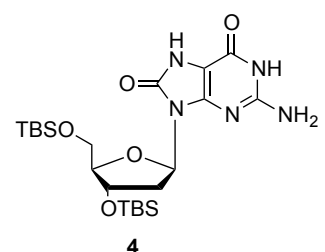

5',3'-O-(bis-tert-butyldimethylsilyl)-8-oxo-2'-dG (4). A mixture of 3 (2.2 g, $7.8 \mathrm{mmol})$, TBDMSCl $(2.5 \mathrm{~g}, 17.1 \mathrm{mmol})$ and imidazole $(1.2 \mathrm{~g}, 17.1 \mathrm{mmol})$ was stirred in $20 \mathrm{~mL}$ of dry DMF at room temperature for $24 \mathrm{~h}$. The reaction was stopped by adding $\mathrm{NaHCO}_{3}$ solution $(50 \mathrm{~mL})$ and extracted with ethyl acetate $(50 \mathrm{~mL})$. The organic layer was washed twice with brine $(50 \mathrm{~mL})$, dried over $\mathrm{MgSO}_{4}$ and the solvent removed in vacuo. The crude product was purified by column chromatography (silica gel, $n$-hexane/ethyl acetate/methanol 2:2:0.4) to give $\mathbf{4}(2.4 \mathrm{~g}, 60 \%)$ as beige solid. ${ }^{1} \mathbf{H}$ 
NMR (300 MHz, DMSO) $\delta 10.72(\mathrm{~s}, 1 \mathrm{H}), 10.59$ (s, 1H), 6.37 (s, 2H), $5.98(\mathrm{t}, J=7.2$ $\mathrm{Hz}, 1 \mathrm{H}), 4.47(\mathrm{t}, J=11.0 \mathrm{~Hz}, 1 \mathrm{H}), 3.77(\mathrm{~m}, 1 \mathrm{H}), 3.56(\mathrm{dd}, J=13.6,8.8 \mathrm{~Hz}, 1 \mathrm{H}), 3.28$ (m, 2H), $1.92(\mathrm{~m}, 1 \mathrm{H}), 0.86$ (s, 18H), -0.03 (s, 12H). ${ }^{13} \mathbf{C}$ NMR (75 MHz, DMSO) $\delta$ 153.1 (C), 151.3 (C), 151.1 (C), 147.3 (C), $98.6(\mathrm{C}), 86.4(\mathrm{CH}), 80.7(\mathrm{CH}), 72.9(\mathrm{CH})$, $63.0\left(\mathrm{CH}_{2}\right), 34.5\left(\mathrm{CH}_{2}\right), 25.9\left(\mathrm{CH}_{3}\right), 17.1(\mathrm{C}),-3.36\left(\mathrm{CH}_{3}\right)$.

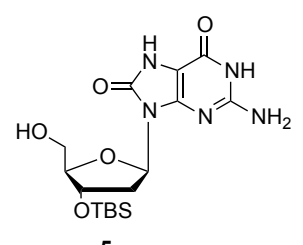

5

3'-O-(tert-butyldimethylsilyl)-8-oxo-2'-dG (5). To a solution of compound $4(1.5 \mathrm{~g}, 2.9 \mathrm{mmol})$ in $\mathrm{CH}_{2} \mathrm{Cl}_{2}(15 \mathrm{~mL})$, a mixture of trifluoroacetic acid and water $(0.6: 1.8 \mathrm{~mL})$ was added, and the reaction was stirred at $0{ }^{\circ} \mathrm{C}$ for $4 \mathrm{~h}$. The reaction was stopped by adding $\mathrm{NaHCO}_{3}$ solution $(10 \mathrm{~mL})$ and extracted with $\mathrm{CH}_{2} \mathrm{Cl}_{2}(10 \mathrm{~mL})$. The organic layer was washed with brine $(10 \mathrm{~mL})$, dried over $\mathrm{MgSO}_{4}$ and the solvent removed in vacuo. The crude product was purified by column chromatography (silica gel, $n$ hexane/ethyl acetate/methanol 2:2:0.5) to give $\mathbf{5}(0.7 \mathrm{~g}, 65 \%)$ as beige solid. ${ }^{1} \mathbf{H}$ NMR (300 MHz, DMSO) $\delta 10.69$ (s, 1H), 10.61 (s, 1H), 6.33 (s, 2H), $5.92(\mathrm{t}, J=7.3 \mathrm{~Hz}$, $1 \mathrm{H}), 4.75(\mathrm{dd}, J=6.8,5.1 \mathrm{~Hz}, 1 \mathrm{H}), 4.50(\mathrm{~m}, 1 \mathrm{H}), 3.71(\mathrm{~m}, 1 \mathrm{H}), 3.47(\mathrm{dt}, J=10.7,5.3$ $\mathrm{Hz}, 1 \mathrm{H}), 3.41(\mathrm{~m}, 1 \mathrm{H}), 3.02(\mathrm{~m}, 1 \mathrm{H}), 1.83(\mathrm{~m}, 1 \mathrm{H}), 0.80(\mathrm{~s}, 9 \mathrm{H}),-0.00(\mathrm{~s}, 6 \mathrm{H}) .{ }^{13} \mathrm{C}$ NMR (75 MHz, DMSO) $\delta 153.1$ (C), 151.5 (C), 151.1 (C), 147.4 (C), 98.5 (C), 87.4 $(\mathrm{CH}), 81.1(\mathrm{CH}), 73.2(\mathrm{CH}), 61.9\left(\mathrm{CH}_{2}\right), 35.6\left(\mathrm{CH}_{2}\right), 25.7\left(\mathrm{CH}_{3}\right), 17.7(\mathrm{C}),-4.8\left(\mathrm{CH}_{3}\right)$.

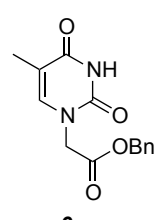

Thymine acetic acid benzyl ester (6). Thymine acetic acid (5.0 g, 27.1 mmol) was dissolved in DMF (50 mL), 1,1'-carbonyldiimidazole $(5.0 \mathrm{~g}$, $31.2 \mathrm{mmol}$ ) was then added. The slurry was stirred at $\mathrm{rt}$ for $30 \mathrm{~min}$ and benzyl alcohol was added $(3.2 \mathrm{~mL}, 31.22 \mathrm{mmol})$. The solution was stirred at $\mathrm{rt}$ for $18 \mathrm{~h}$. Then, $\mathrm{H}_{2} \mathrm{O}(100 \mathrm{~mL})$ was added at $0{ }^{\circ} \mathrm{C}$ and the product was isolated as a white solid 6 (4.8 g, 65\%) upon filtration. ${ }^{1} \mathbf{H}$ NMR (300 MHz, DMSO) $\delta 11.42$ (s, 1H), 7.53 (s, 1H), 7.47 (m, 5H), 5.19 (s, 2H), 4.55 (s, 2H), 1.76 (s, 3H). ${ }^{13}$ C NMR (75 MHz, DMSO) $\delta 168.1$ (C), 164.2 (C), 150.9 (C), 141.5 (CH), 135.5 (C), $128.4(\mathrm{CH})$, $128.2(\mathrm{CH}), 127.9(\mathrm{CH}), 108.6(\mathrm{C}), 66.4\left(\mathrm{CH}_{2}\right), 48.4\left(\mathrm{CH}_{2}\right), 11.8\left(\mathrm{CH}_{3}\right)$. 
Chapter 5: Photorepair of Thymine Derived Cyclobutanes and Oxetanes by 8-oxoGuanine

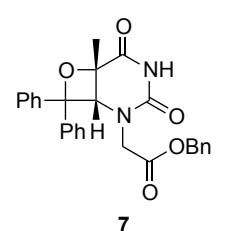

Thymine oxetane acetic acid benzyl ester (7). In a Pyrex vessel $(\lambda$ $>290 \mathrm{~nm}$ ), the benzyl ester 6 (4.5 g, $16.4 \mathrm{mmol})$ was dissolved in acetonitrile $(444 \mathrm{~mL})$ under sonication. Benzophenone $(5.9 \mathrm{~g}, 32.8$ $\mathrm{mmol}$ ) was added and the solution was degassed by bubbling $\mathrm{N}_{2}$ for $0.5 \mathrm{~h}$. The solution was irradiated for $16 \mathrm{~h}$ with a medium pressure mercury lamp (125 W). Acetonitrile was removed in vacuo and the product was isolated by flash chromatography (silica gel, $n$-hexane/ethyl acetate $4: 1$ to 1:1). Recrystallisation from $n$ hexane/ethyl acetate (4:1) gave 7 (1.6 g, 22\%) as a white solid. ${ }^{1} \mathbf{H}$ NMR (300 MHz, DMSO) $\delta 10.50$ (s, 1H), $7.49-7.16$ (m, 15H), $5.29-5.05$ (m, 2H), $4.99(\mathrm{~s}, 1 \mathrm{H}), 4.40$ $(\mathrm{d}, J=17.4 \mathrm{~Hz}, 1 \mathrm{H}), 4.10(\mathrm{~d}, J=17.5 \mathrm{~Hz}, 1 \mathrm{H}), 1.51$ (s, 3H). ${ }^{13} \mathbf{C}$ NMR (75 MHz, DMSO) $\delta 170.0(\mathrm{C}), 168.4(\mathrm{C}), 151.2(\mathrm{C}), 144.3(\mathrm{C}), 139.5(\mathrm{C}), 135.6(\mathrm{C}), 128.4(\mathrm{CH})$, $128.3(\mathrm{CH}), 128.2(\mathrm{CH}), 128.1(\mathrm{CH}), 128.0(\mathrm{CH}), 127.5(\mathrm{CH}), 125.4(\mathrm{CH}), 125.0(\mathrm{CH})$, $90.9(\mathrm{C}), 76.1(\mathrm{C}), 66.3\left(\mathrm{CH}_{2}\right), 64.9(\mathrm{CH}), 48.1\left(\mathrm{CH}_{2}\right), 23.1\left(\mathrm{CH}_{3}\right)$.

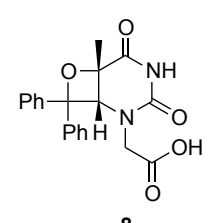

Thymine oxetane acetic acid (8). To a stirred solution of 7 (500 mg, $1.1 \mathrm{mmol})$ in glacial acetic acid $(5 \mathrm{~mL})$ was added 10\% Pd/C (13 mg, $0.1 \mathrm{mmol})$. The solution was stirred under $\mathrm{H}_{2}$ atmosphere for $4 \mathrm{~h}$ at atmospheric pressure. The reaction mixture was filtered through celite and the filter cake was washed with hot acetic acid $(20 \mathrm{~mL})$. The solvent was removed in vacuo to afford 8 (300 mg, 75\%) as white solid. ${ }^{1} \mathbf{H}$ NMR (300 MHz, DMSO) $\delta$ 12.97 (bs, 1H), 10.42 (s, 1H), $7.48-7.17(\mathrm{~m}, 10 \mathrm{H}), 4.92(\mathrm{~s}, 1 \mathrm{H}), 4.25$ (d, $J=17.5 \mathrm{~Hz}$, 1H), $3.85(\mathrm{~d}, J=17.5 \mathrm{~Hz}, 1 \mathrm{H}), 1.54(\mathrm{~s}, 3 \mathrm{H}) .{ }^{13} \mathrm{C}$ NMR (75 MHz, DMSO) $\delta 170.1$ (C), $151.3(\mathrm{C}), 144.3(\mathrm{C}), 139.6(\mathrm{C}), 128.4(\mathrm{CH}), 128.1(\mathrm{CH}), 127.5(\mathrm{CH}), 125.4(\mathrm{CH})$, $124.5(\mathrm{CH}), 90.9(\mathrm{C}), 76.1(\mathrm{C}), 65.1(\mathrm{CH}), 47.9\left(\mathrm{CH}_{2}\right), 23.2\left(\mathrm{CH}_{3}\right)$.

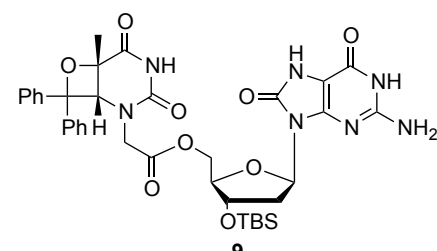

9

5'-thymine oxetane-3'-O-(tert-butyldimethylsilyl)8-oxo-2'-dG (9). Oxetane 8 (120 mg, $0.3 \mathrm{mmol})$, EDC (65 $\mu \mathrm{L}, 0.4 \mathrm{mmol})$, TBTU (117 mg, $0.4 \mathrm{mmol}$ ), DMAP (4 mg, $0.03 \mathrm{mmol}$ ) were dissolved in dry DMF $(6 \mathrm{~mL})$ 
Chapter 5: Photorepair of Thymine Derived Cyclobutanes and Oxetanes by 8-oxoGuanine

and stirred at $0{ }^{\circ} \mathrm{C}$ for $30 \mathrm{~min}$. Then, $5(132 \mathrm{mg}, 0.3 \mathrm{mmol})$ was added and the solution was stirred for $20 \mathrm{~h}$ at $\mathrm{rt}$. The reaction mixture was diluted with ethyl acetate $(20 \mathrm{~mL})$, washed with water $(3 \times 20 \mathrm{~mL})$, dried over $\mathrm{MgSO}_{4}$, filtered and the solvent was removed in vacuo. The crude product was purified by flash chromatography (silica gel, $n-$ hexane/ethyl acetate/methanol 2:2:0.5) to afford $\mathbf{9}(136 \mathrm{mg}, 55 \%)$ as a white solid. ${ }^{1} \mathbf{H}$ NMR (300 MHz, DMSO) $\delta 10.67$ (s, 1H), 10.57 (d, $J=3.0 \mathrm{~Hz}, 1 \mathrm{H}), 10.40$ (d, $J=$ $3.5 \mathrm{~Hz}, 1 \mathrm{H}), 7.37-7.08(\mathrm{~m}, 10 \mathrm{H}), 6.32(\mathrm{~s}, 2 \mathrm{H}), 5.94(\mathrm{t}, J=7.1 \mathrm{~Hz}, 1 \mathrm{H}), 4.86(\mathrm{~d}, J=$ $5.8 \mathrm{~Hz}, 1 \mathrm{H}), 4.52(\mathrm{~m}, 1 \mathrm{H}), 4.34-4.16(\mathrm{~m}, 2 \mathrm{H}), 4.04(\mathrm{dd}, J=11.3,7.4 \mathrm{~Hz}, 1 \mathrm{H}), 3.92$ $(\mathrm{dd}, J=17.8,6.8 \mathrm{~Hz}, 1 \mathrm{H}), 3.81(\mathrm{dd}, J=11.4,7.4 \mathrm{~Hz}, 1 \mathrm{H}), 2.99(\mathrm{~m}, 1 \mathrm{H}), 1.94(\mathrm{~m}, 1 \mathrm{H})$, 1.47 (s, 3H), 0.79 (s, 9H), -0.00 (s, 6H). ${ }^{13}$ C NMR (75 MHz, DMSO) $\delta 168.9$ (C), 168.3 (C), 153.1 (C) 151.3 (C), 151.2 (C), 147.0 (C), 144.3 (C), 139.5 (C), $128.3(\mathrm{CH})$, $128.1(\mathrm{CH}), 127.5(\mathrm{CH}), 125.4(\mathrm{CH}), 125.0(\mathrm{CH}), 98.5(\mathrm{C}), 90.9(\mathrm{C}), 83.5(\mathrm{CH}), 80.7$ $(\mathrm{CH}), 76.1(\mathrm{C}), 72.8(\mathrm{CH}), 64.9(\mathrm{CH}), 64,9\left(\mathrm{CH}_{2}\right), 47.7\left(\mathrm{CH}_{2}\right), 35.8\left(\mathrm{CH}_{2}\right), 25.6\left(\mathrm{CH}_{3}\right)$, $23.0\left(\mathrm{CH}_{3}\right), 17.6(\mathrm{C}),-4.84\left(\mathrm{CH}_{3}\right)$.

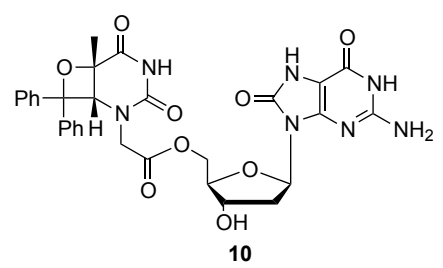

(silica gel, $\mathrm{CHCl}_{3} / \mathrm{MeOH}$ 5:0.5 to 5:0.1) to afford $10(140 \mathrm{mg}, 68 \%)$ as a white solid. $7.10(\mathrm{~m}, 10 \mathrm{H}), 6.37(\mathrm{~s}, 2 \mathrm{H}), 5.96(\mathrm{t}, J=7.0 \mathrm{~Hz}, 1 \mathrm{H}), 5.28-5.11(\mathrm{~m}, 1 \mathrm{H}), 4.87(\mathrm{~d}, J=$ $0.8 \mathrm{~Hz}, 1 \mathrm{H}), 4.44-4.23(\mathrm{~m}, 3 \mathrm{H}), 4.09-3.98(\mathrm{~m}, 1 \mathrm{H}), 3.91(\mathrm{dd}, J=17.5,6.9 \mathrm{~Hz}, 1 \mathrm{H})$, $3.79(\mathrm{dt}, J=7.8,4.1 \mathrm{~Hz}, 1 \mathrm{H}), 2.89(\mathrm{dd}, J=10.8,6.6 \mathrm{~Hz}, 1 \mathrm{H}), 2.04-1.92(\mathrm{~m}, 1 \mathrm{H})$, 1.48 (s, 3H). ${ }^{13}$ C NMR (75 MHz, DMSO) $\delta 170.1$ (C), 168.4 (C), 153.1 (C), 151.4 (C), $151.2(\mathrm{C}), 147.1(\mathrm{C}), 144.3(\mathrm{C}), 139.5(\mathrm{C}), 128.3(\mathrm{CH}), 128.1(\mathrm{CH}), 127.5(\mathrm{CH})$, 
Chapter 5: Photorepair of Thymine Derived Cyclobutanes and Oxetanes by 8-oxoGuanine

$125.4(\mathrm{CH}), 125.0(\mathrm{CH}), 124.9(\mathrm{CH}), 98.5(\mathrm{C}), 90.9(\mathrm{C}), 83.4(\mathrm{CH}), 80.7(\mathrm{CH}), 76.1$ (C), $71.0(\mathrm{CH}), 65.6\left(\mathrm{CH}_{2}\right), 64.9(\mathrm{CH}), 47.8\left(\mathrm{CH}_{2}\right), 35.5\left(\mathrm{CH}_{2}\right), 23.1\left(\mathrm{CH}_{3}\right)$.

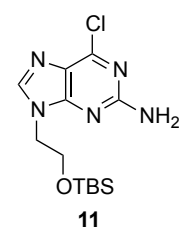

2-Amino-6-chloro-9-(2-ethyloxy-tert-butyldimethylsilane) purine

(11). To an ice cold solution of 2-amino-6-chloro purine (2.8 g, 16.5 $\mathrm{mmol})$ in anhydrous DMF $(25 \mathrm{~mL})$ was added $\mathrm{NaH}(0.4 \mathrm{~g}, 17.9 \mathrm{mmol})$. After $30 \mathrm{~min}$, 2-bromoethoxy-tert-butyldimethylsilane (4.3 g, $18.1 \mathrm{mmol})$ was added and stirred $24 \mathrm{~h}$ at $\mathrm{rt}$ under nitrogen. Then, $\mathrm{H}_{2} \mathrm{O}(25 \mathrm{~mL})$ was poured and the mixture was filtrated and washed with cold water. The crude product was purified by column chromatography (silica gel, $n$-hexane/ethyl acetate 2:3) to give 11 ( $2.4 \mathrm{~g}$, 51\%) as white solid. ${ }^{1} \mathbf{H}$ NMR (300 MHz, DMSO) $\delta 8.05$ (s, 1H), 6.89 (s, 2H), 4.16 $(\mathrm{t}, J=12 \mathrm{~Hz}, 2 \mathrm{H}), 3.89$ (t, $J=12 \mathrm{~Hz}, 2 \mathrm{H}), 0.75$ (s, 9H), -0.15 (s, 6H). ${ }^{13} \mathbf{C}$ NMR (75 MHz, DMSO) $\delta 159.9$ (C), $154.2(\mathrm{C}), 149.2(\mathrm{C}), 143.8(\mathrm{CH}), 123.4(\mathrm{C}), 60.3\left(\mathrm{CH}_{2}\right)$, $45.4\left(\mathrm{CH}_{2}\right), 25.9\left(\mathrm{CH}_{3}\right), 17.6(\mathrm{C}),-5.80\left(\mathrm{CH}_{3}\right)$. HRMS (ESI): $\mathrm{m} / \mathrm{z}$ calcd for $\mathrm{C}_{13} \mathrm{H}_{23} \mathrm{~N}_{5} \mathrm{OSiCl}[\mathrm{M}+\mathrm{H}]^{+}$328.1344, found 328.1360.

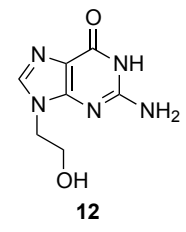

9-(2-ethyloxy-tert-butyldimethylsilane) guanine (12). A solution of $11(2.4 \mathrm{~g}, 7.33 \mathrm{mmol})$ in $2 \mathrm{M} \mathrm{HCl}(22 \mathrm{~mL})$ was heated under reflux for 2 h. The solvent was neutralized and cooled with ice. The solid was filtered off and washed with cold $\mathrm{H}_{2} \mathrm{O}$ to give pure $12(1.3 \mathrm{~g}, 87 \%)$. ${ }^{1} \mathbf{H}$ NMR (300 MHz, DMSO) $\delta 10.50(\mathrm{~s}, 1 \mathrm{H}), 7.61(\mathrm{~s}, 1 \mathrm{H}), 6.40(\mathrm{~s}, 2 \mathrm{H}), 4.96(\mathrm{t}, \mathrm{J}=9$ $\mathrm{Hz} 1 \mathrm{H}), 3.96(\mathrm{t}, J=12 \mathrm{~Hz}, 2 \mathrm{H}), 3.69-3.63(\mathrm{~m}, 2 \mathrm{H}) .{ }^{13} \mathbf{C}$ NMR (75 MHz, DMSO) $\delta$ 156.9 (C), $153.4(\mathrm{C}), 151.3(\mathrm{C}), 137.9(\mathrm{CH}), 116.3(\mathrm{C}), 59.1\left(\mathrm{CH}_{2}\right), 45.4\left(\mathrm{CH}_{2}\right)$. HRMS (ESI): $m / z$ calcd for $\mathrm{C}_{7} \mathrm{H}_{10} \mathrm{~N}_{5} \mathrm{O}_{2}[\mathrm{M}+\mathrm{H}]^{+} 196.0825$, found 196.0834 .

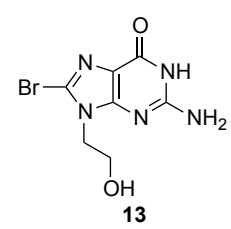

9-(2-ethyloxy-tert-butyldimethylsilane)-8-bromoguanine (13). In a round bottom flask, $12(1.3 \mathrm{~g}, 6.1 \mathrm{mmol})$ was dissolved in $62 \mathrm{~mL}$ $\mathrm{MeCN}$ and $15 \mathrm{~mL} \mathrm{H}_{2} \mathrm{O}$ and NBS (1.6 g, $9.1 \mathrm{mmol}$ ) was added. The suspension was stirred for $30 \mathrm{~min}$ at $\mathrm{rt}$ and subsequently evaporated to dryness. The residual solid was taken up in $20 \mathrm{~mL}$ acetone and stirred for $30 \mathrm{~min}$ at 
room temperature. Subsequently, the mixture was filtrated and washed with cold acetone and dried to yield $\mathbf{1 3}(0.85 \mathrm{~g}, 65 \%)$ as an beige solid. ${ }^{1} \mathbf{H}$ NMR (300 MHz, DMSO) $\delta 10.64$ (s, 1H), 6.55 (s, 2H), 4.98 (t, J = $10 \mathrm{~Hz}, 1 \mathrm{H}), 3.97$ (t, J = $12 \mathrm{~Hz}, 2 \mathrm{H})$, 3.68 - 3.62 (m, 2H). ${ }^{13}$ C NMR (75 MHz, DMSO) $\delta 155.7$ (C), 153.9 (C), 152.5 (C), 121.4 (C), 116.6 (C), $58.8\left(\mathrm{CH}_{2}\right), 46.0\left(\mathrm{CH}_{2}\right)$. HRMS (ESI): $\mathrm{m} / \mathrm{z}$ calcd for $\mathrm{C}_{7} \mathrm{H}_{9} \mathrm{~N}_{5} \mathrm{O}_{2} \mathrm{Br}[\mathrm{M}+\mathrm{H}]^{+}$273.9952, found 273.9940 .<smiles>COCCn1c(=O)[nH]c2nc(N)[nH]c(=O)c21</smiles>

9-(2-hydroxyethyl)-8-oxoguanine (14). Compound 13 (0.85 g, 3.1 mmol) was dissolved in a solution of sodium acetate $(2.5 \mathrm{~g}, 31.14$ $\mathrm{mmol})$ in glacial acetic acid $(113 \mathrm{~mL})$. The reaction mixture was heated at $130^{\circ} \mathrm{C}$ for $7 \mathrm{~h}$. Acetic acid was evaporated and a residue was codistilled with water $(3 \times 20 \mathrm{~mL})$. The residual solid was taken up in aqueous sodium hydroxide $(0.1 \mathrm{M}, 10 \mathrm{~mL})$ and heated at reflux $10 \mathrm{~min}$. Then, it was acidified to $\mathrm{pH} 7$ in an ice bath and the resulting precipitated filtered off, washed with water to give $\mathbf{1 4}$ (0.3 g, 46\%) as beige solid. ${ }^{1} \mathbf{H}$ NMR (300 MHz, DMSO) ${ }^{1} \delta 10.58$ (s, $\left.1 \mathrm{H}\right), 10.49$ (s, $1 \mathrm{H}), 6.43(\mathrm{~s}, 2 \mathrm{H}), 4.81(\mathrm{t}, \mathrm{J}=10 \mathrm{~Hz}, 1 \mathrm{H}), 3.68-3.63(\mathrm{~m}, 2 \mathrm{H}), 3.60-3.57(\mathrm{~m}, 2 \mathrm{H})$. ${ }^{13}$ C NMR (75 MHz, DMSO) $\delta 153.7$ (C), 152.5 (C), 150.7 (C), 147.9 (C), 97.7 (C), $58.5\left(\mathrm{CH}_{2}\right), 41.5\left(\mathrm{CH}_{2}\right)$. HRMS (ESI): $m /$ z calcd for $\mathrm{C}_{7} \mathrm{H}_{10} \mathrm{~N}_{5} \mathrm{O}_{3}[\mathrm{M}+\mathrm{H}]^{+} 212.0780$, found 212.0784 .

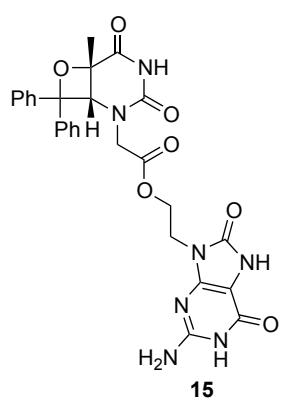

9-(2-(thymine oxetane) ethyl)-8-oxoguanine (15). Oxetane carboxylic acid 8 (192 mg, $0.5 \mathrm{mmol})$, EDC (102 $\mu \mathrm{L}, 0.6 \mathrm{mmol})$, TBTU (186 mg, $0.6 \mathrm{mmol}$ ), DMAP (6.4 mg, $0.05 \mathrm{mmol}$ ) were dissolved in dry DMF $(5 \mathrm{~mL})$ and stirred at $0{ }^{\circ} \mathrm{C}$ for $30 \mathrm{~min}$. Then, $14(100 \mathrm{mg}, 0.5 \mathrm{mmol})$ was added and the solution was stirred for $24 \mathrm{~h}$ at $\mathrm{rt}$. The reaction mixture was diluted with $\mathrm{H}_{2} \mathrm{O}$ $(10 \mathrm{~mL})$ and cooled with ice. The solid was filtered off and washed with cold water. The crude product was purified by column chromatography (silica gel, $n$-hexane/ethyl acetate/methanol 2:2:0.5) to give 15 (88 $\mathrm{mg}, 33 \%)$ as beige 
Chapter 5: Photorepair of Thymine Derived Cyclobutanes and Oxetanes by 8-oxoGuanine

solid. 1H NMR (300 MHz, DMSO) $\delta 10.71$ (s, 1H), 10.62 (s, 1H), 10.48 (s, 1H), 7.29 (m, 10H), 6.49 (s, 2H), 4.94 (s, 1H), $4.30-3.88(\mathrm{~m}, 3 \mathrm{H}), 3.88-1.57$ (m, 3H), 1.57 (s, 3H). ${ }^{13 C}$ NMR (75 MHz, DMSO) $\delta 170.0$ (C), 168.4 (C), 153.5 (C), 152.3 (C), 151.2 (C), $147.9(\mathrm{C}), 144.1(\mathrm{C}), 139.5(\mathrm{C}), 128.4(\mathrm{CH}), 128.1(\mathrm{CH}), 127.5(\mathrm{CH}), 125.4(\mathrm{CH})$, $125.1(\mathrm{CH}), 98.3(\mathrm{C}), 90.9(\mathrm{C}), 76.0(\mathrm{C}), 64.8(\mathrm{CH}), 62.3\left(\mathrm{CH}_{2}\right), 47.8\left(\mathrm{CH}_{2}\right), 38.0\left(\mathrm{CH}_{2}\right)$, $23.1\left(\mathrm{CH}_{3}\right)$. HRMS (ESI): $m / z$ calcd for $\mathrm{C}_{27} \mathrm{H}_{26} \mathrm{~N}_{7} \mathrm{O}_{7}[\mathrm{M}+\mathrm{H}]^{+}$560.1882, found 560.1894 .

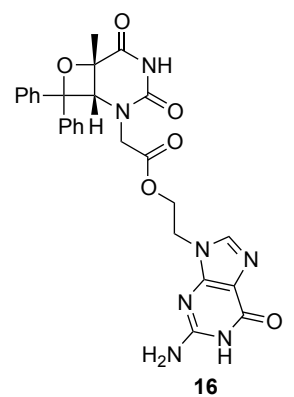

9-(2-(thymine oxetane) ethyl)-guanine (16). Oxetane carboxylic acid 8 (100 mg, $0.3 \mathrm{mmol})$, EDC (53 $\mu \mathrm{L}, 0.3 \mathrm{mmol})$, TBTU (96 mg, $0.3 \mathrm{mmol}$ ), DMAP (3.3 mg, $0.03 \mathrm{mmol}$ ) were dissolved in dry DMF $(2.7 \mathrm{~mL})$ and stirred at $0{ }^{\circ} \mathrm{C}$ for $30 \mathrm{~min}$. Then, $12(53$ $\mathrm{mg}, 0.3 \mathrm{mmol}$ ) was added and the solution was stirred for $24 \mathrm{~h}$ at rt. The reaction mixture was diluted with $\mathrm{H}_{2} \mathrm{O}(5 \mathrm{~mL})$ and cooled with ice. The solid was filtered off and washed with cold water to

give 16 (71 mg, 48\%) as white solid. ${ }^{1} \mathbf{H}$ NMR (300 MHz, DMSO) $\delta 10.55$ (s, $1 \mathrm{H}$ ), 10.49 (s, 1H), 7.69 (s, 1H), $7.44-7.19$ (m, 10H), 6.43 (s, 2H), 4.92 (s, 1H), $4.49-4.16$ (m, 5H), $4.01-3.95(\mathrm{~m}, 1 \mathrm{H}), 1.57$ (s, 3H). ${ }^{13} \mathbf{C}$ NMR (75 MHz, DMSO) $\delta 169.8(\mathrm{C})$, $168.3(\mathrm{C}), 156.6(\mathrm{C}), 153.4(\mathrm{C}), 150.9$ (C), 144.4 (C), 139.9 (C), 137.2 (C), $128.4(\mathrm{CH})$, $128.1(\mathrm{CH}), 127.5(\mathrm{CH}), 125.4(\mathrm{CH}), 125.0(\mathrm{CH}), 91.1(\mathrm{C}), 75.9(\mathrm{C}), 65.1(\mathrm{CH}), 62.7$ $\left(\mathrm{CH}_{2}\right), 47.8\left(\mathrm{CH}_{2}\right), 41.9\left(\mathrm{CH}_{2}\right), 22.9\left(\mathrm{CH}_{3}\right)$. (C8-H signat at ca. $140 \mathrm{ppm}$ has not been detected due to high signal-noise) HRMS (ESI): $m / z$ calcd for $\mathrm{C}_{27} \mathrm{H}_{26} \mathrm{~N}_{7} \mathrm{O}_{6}[\mathrm{M}+\mathrm{H}]^{+}$ 544.1943, found 544.1945.

Methyl 2-(thymin-1-yl)acetate (17). To a stirred solution of thymine acetic acid $(5 \mathrm{~g}, 27.1 \mathrm{mmol})$ in $\mathrm{MeOH}(200 \mathrm{~mL}), \mathrm{H}_{2} \mathrm{SO}_{4}(1 \mathrm{~mL})$ was added. The reaction was heated to reflux overnight. The solvent was evaporated under pressure, diluted with $\mathrm{H}_{2} \mathrm{O}(100 \mathrm{~mL})$ and cooled with ice. The solid was filtered off and washed well with cold $\mathrm{H}_{2} \mathrm{O}$ to give pure 17 (4.2 g, 78\%) 
as white solid. ${ }^{1} \mathbf{H}$ NMR (300 MHz, DMSO) $\delta 11.39$ (s, 1H), 7.49 (s, 1H), 4.47 (s, 2H), 3.68 (s, 3H), 1.75 (s, 3H). ${ }^{13}$ C NMR (75 MHz, DMSO) $\delta 168.7$ (C), 164.2 (C), $150.9(\mathrm{C}), 141.7(\mathrm{CH}), 108.5(\mathrm{C}), 52.3\left(\mathrm{CH}_{3}\right), 48.4\left(\mathrm{CH}_{2}\right), 11.8\left(\mathrm{CH}_{3}\right)$.

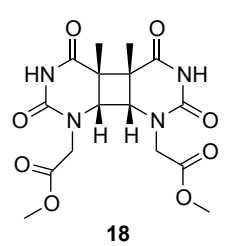

Cis-syn-thymine dimer ester (18). The methyl ester 17 (4 g, 20.2 $\mathrm{mmol})$ was dissolved in acetone/acetonitrile $(500 \mathrm{~mL}, 1: 4)$ and the solution was degassed for $30 \mathrm{~min}$. The solution was irradiated for 72 $\mathrm{h}$ with a medium pressure mercury lamp (125 W) in a Pyrex vessel.

The reaction mixture was filtrated and evaporated to dryness in vacuo. The product was isolated by flash chromatography (silica gel, $\mathrm{CHCl}_{3} / \mathrm{MeOH}$ 5:0.15) as white solid 18 (0.3 g, 3.8\%). ${ }^{1} \mathbf{H}$ NMR (300 MHz, DMSO) $\delta 10.51$ (s, 2H), 4.21 (d, $J=17.4 \mathrm{~Hz}$, 2H), $3.96(\mathrm{~s}, 2 \mathrm{H}), 3.85$ (d, $J=17.4 \mathrm{~Hz}, 2 \mathrm{H}), 3.65$ (s, 6H), 1.35 (s, 6H). ${ }^{13} \mathbf{C}$ NMR (75 MHz, DMSO) $\delta 170.4$ (2C), 169.3 (2C), $152.2(2 \mathrm{C}), 59.5(2 \mathrm{CH}), 52.1\left(2 \mathrm{CH}_{3}\right), 47.1$ $\left(2 \mathrm{CH}_{2}\right), 46.2(2 \mathrm{C}), 18.1\left(2 \mathrm{CH}_{3}\right)$.

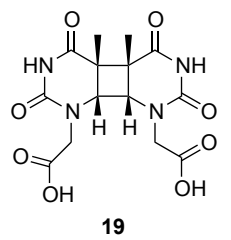

Cis-syn thymine dimer acetic acid (19). The diester 18 (200 mg, $0.5 \mathrm{mmol})$ was dissolved in $5 \mathrm{M}$ hydrochloride $(5 \mathrm{~mL})$. The reaction mixture was refluxed for $30 \mathrm{~min}$ and then the reaction solution was concentrated in vacuo. The product was washed with $\mathrm{Et}_{2} \mathrm{O}$ and dried in vacuo to yield a white solid $19(0.1 \mathrm{~g}, 62 \%) .{ }^{1} \mathbf{H}$ NMR (300 MHz, DMSO) $\delta 12.82$ (s, 2H), 10.48 (s, 2H), 4.17 (d, $J=17.4 \mathrm{~Hz}, 2 \mathrm{H}), 3.94(\mathrm{~s}, 2 \mathrm{H}), 3.70$ (d, $J=17.4 \mathrm{~Hz}$, 2H), 1.30 (s, 6H). ${ }^{13}$ C NMR (75 MHz, DMSO) $\delta 170.7$ (2C), 170.1 (2C), 152.2 (2C), $59.4(2 \mathrm{CH}), 47.2\left(2 \mathrm{CH}_{2}\right), 46.3(2 \mathrm{C}), 18.2\left(2 \mathrm{CH}_{3}\right)$. 


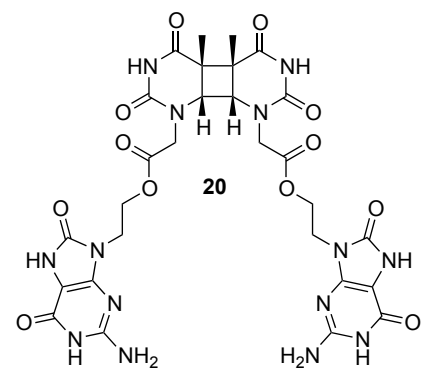

9-(2-(thymine dimer) ethyl)-8-oxoguanine (20). Cissyn-thymine dimer carboxylic 19 (97 mg, $0.3 \mathrm{mmol}$ ), EDC $(102 \mu \mathrm{L}, 0.6 \mathrm{mmol})$, TBTU $(186 \mathrm{mg}, 0.6 \mathrm{mmol})$, DMAP (6.4 mg, $0.05 \mathrm{mmol}$ ) were dissolved in dry DMF $(5 \mathrm{~mL})$ and stirred at $0{ }^{\circ} \mathrm{C}$ for $30 \mathrm{~min}$. Then, $14(100 \mathrm{mg}$, $0.5 \mathrm{mmol}$ ) was added and the solution was stirred for 24 $\mathrm{h}$ at rt. The unreacted 14 was filtered and the filtrate was diluted with $\mathrm{H}_{2} \mathrm{O}(10 \mathrm{~mL})$ and cooled with ice. The solid was filtered off and washed with cold water to give 20 (50 mg, 25\%) as beige solid. ${ }^{1}$ H NMR (300 MHz, DMSO) $\delta 10.74$ (s, 4H), 10.53 (s, 2H), $6.52(\mathrm{~s}, 4 \mathrm{H}), 4.39-4.21(\mathrm{~m}, 6 \mathrm{H}), 3.92-3.68(\mathrm{~m}, 8 \mathrm{H}), 1.30(\mathrm{~s}, 6 \mathrm{H}) .{ }^{13} \mathbf{C}$ NMR (75 MHz, DMSO) $\delta 170.4$ (2C), 168.7 (2C), 153.6 (2C), 152.4 (2C), 152.1 (2C), 151.1 (2C), $147.9(2 \mathrm{C}), 98.6(2 \mathrm{C}), 62.2(2 \mathrm{CH}), 59.0\left(2 \mathrm{CH}_{2}\right), 47.1\left(2 \mathrm{CH}_{2}\right), 46.3(2 \mathrm{C}), 37.9$ $\left(2 \mathrm{CH}_{2}\right), 18.3\left(2 \mathrm{CH}_{3}\right)$. HRMS (ESI): $m / z$ calcd for $\mathrm{C}_{28} \mathrm{H}_{31} \mathrm{~N}_{14} \mathrm{O}_{12}[\mathrm{M}+\mathrm{H}]^{+} 755.2270$, found 755.2246 .

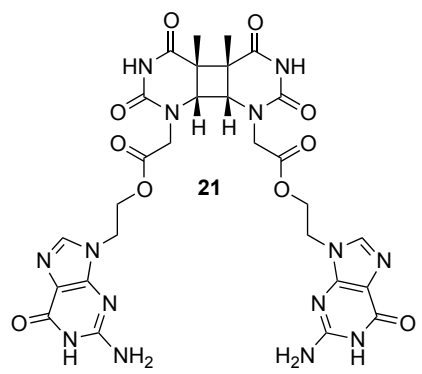

9-(2-(thymine dimer) ethyl)-guanine (21). Cis-synthymine dimer carboxylic 19 (65 $\mathrm{mg}, 0.2 \mathrm{mmol})$, EDC (34 $\mu \mathrm{L}, 0.4 \mathrm{mmol})$, TBTU (124 mg, $0.4 \mathrm{mmol})$, DMAP (4.3 mg, $0.03 \mathrm{mmol})$ were dissolved in dry DMF $(2 \mathrm{~mL})$ and stirred at $0{ }^{\circ} \mathrm{C}$ for $30 \mathrm{~min}$. Then, $12(68 \mathrm{mg}, 0.4$ mmol) was added and the solution was stirred for $24 \mathrm{~h}$ at $\mathrm{rt}$. The reaction mixture was diluted with $\mathrm{H}_{2} \mathrm{O}(4 \mathrm{~mL})$ and cooled with ice. The solid was filtered off and washed with cold water to give 21 (63 $\mathrm{mg}, 50 \%)$ as white solid. 1H NMR (300 MHz, DMSO) $\delta 10.58$ (s, 2H), 10.54 (s, 2H), 7.68 (s, 2H), 6.46 $(\mathrm{s}, 4 \mathrm{H}), 4.42-4.33(\mathrm{~m}, 4 \mathrm{H}), 4.27-4.18(\mathrm{~m}, 6 \mathrm{H}), 3.88-3.76(\mathrm{~m}, 4 \mathrm{H}), 1.29(\mathrm{~s}, 6 \mathrm{H}) .{ }^{13} \mathrm{C}$ NMR (75 MHz, DMSO) $\delta 170.4$ (2C), 168.6 (2C), 156.6 (2C), 153.7 (2C), 152.2 (2C), $150.9(2 \mathrm{C}), 137.6(2 \mathrm{CH}), 116.6(2 \mathrm{C}), 62.7\left(2 \mathrm{CH}_{2}\right), 59.4(2 \mathrm{CH}), 47.1\left(2 \mathrm{CH}_{2}\right), 45.9$ 
(2C), $41.7\left(2 \mathrm{CH}_{2}\right), 17.9\left(2 \mathrm{CH}_{3}\right)$. HRMS (ESI): $m / z$ calcd for $\mathrm{C}_{28} \mathrm{H}_{31} \mathrm{~N}_{14} \mathrm{O}_{10}[\mathrm{M}+\mathrm{H}]^{+}$ 723.2335 , found 723.2348 .

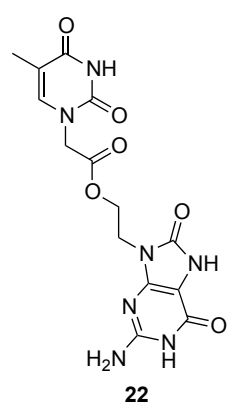

9-(2-(thymine) ethyl)-8-oxo-guanine (22). Thymine acetic acid (100 mg, $0.3 \mathrm{mmol})$, EDC $(25.5 \mu \mathrm{L}, 0.3 \mathrm{mmol})$, TBTU (93 mg, 0.3 mmol), DMAP (4.3 mg, $0.03 \mathrm{mmol})$ were dissolved in dry DMF (2 $\mathrm{mL}$ ) and stirred at $0{ }^{\circ} \mathrm{C}$ for $30 \mathrm{~min}$. Then, $14(51 \mathrm{mg}, 0.3 \mathrm{mmol})$ was added and the solution was stirred for $24 \mathrm{~h}$ at $\mathrm{rt}$. The reaction mixture was diluted with $\mathrm{H}_{2} \mathrm{O}(4 \mathrm{~mL})$ and cooled with ice. The solid was filtered off and washed with cold water to give $22(45 \mathrm{mg}, 40 \%)$ as white solid. ${ }^{1} \mathbf{H}$ NMR (300 MHz, DMSO-d $\left.\mathbf{d}_{\mathbf{6}}\right) \boldsymbol{\delta} 11.39$ (s, 1H), 10.60 (s, 1H), 7.37 (s, $1 \mathrm{H}), 6.49(\mathrm{~s}, 2 \mathrm{H}), \delta 4.41(\mathrm{~s}, 2 \mathrm{H}), 4.32(\mathrm{t}, J=4.2 \mathrm{~Hz}, 2 \mathrm{H}), 3.86(\mathrm{t}, J=4.9 \mathrm{~Hz}, 2 \mathrm{H}), 1.74$ $(\mathrm{s}, 3 \mathrm{H})$.

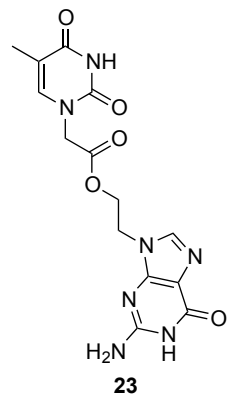

9-(2-(thymine) ethyl)-guanine (23). Thymine acetic acid (100 mg, $0.3 \mathrm{mmol})$, EDC $(25.5 \mu \mathrm{L}, 0.3 \mathrm{mmol})$, TBTU (93 $\mathrm{mg}, 0.3 \mathrm{mmol}$ ), DMAP (4.3 mg, $0.03 \mathrm{mmol})$ were dissolved in dry DMF $(2 \mathrm{~mL})$ and stirred at $0{ }^{\circ} \mathrm{C}$ for $30 \mathrm{~min}$. Then, $12(51 \mathrm{mg}, 0.3 \mathrm{mmol})$ was added and the solution was stirred for $24 \mathrm{~h}$ at $\mathrm{rt}$. The reaction mixture was diluted with $\mathrm{H}_{2} \mathrm{O}(4 \mathrm{~mL})$ and cooled with ice. The solid was filtered off and washed with cold water to give $23(54 \mathrm{mg}, 50 \%)$ as white solid. ${ }^{1 H}$ NMR (300 MHz, DMSO-d6) $\delta 11.41$ (s, 1H), 10.56 (s, 1H), 7.67 (s, 1H), $7.42(\mathrm{~s}, 1 \mathrm{H}), 6.46(\mathrm{~s}, 2 \mathrm{H}), 4.56-4.33(\mathrm{~m}, 4 \mathrm{H}), 4.21$ (t, $J=4.3 \mathrm{~Hz}, 2 \mathrm{H}), 1.75(\mathrm{~s}, 3 \mathrm{H})$. 13C NMR (75 MHz, DMSO-d d $_{\text {) }} \delta 168.0$ (C), 164.2 (C), 156.7 (C), 153.6 (C), 151.2 (C), $150.9(\mathrm{C}), 141.3(\mathrm{CH}), 137.5(\mathrm{CH}), 116.5(\mathrm{C}), 108.7(\mathrm{C}), 63.3\left(\mathrm{CH}_{2}\right), 48.3\left(\mathrm{CH}_{2}\right)$, $41.6\left(\mathrm{CH}_{2}\right), 11.8\left(\mathrm{CH}_{3}\right)$.

\subsubsection{NMR spectra $\left({ }^{1} \mathrm{H}, \mathrm{DEPT},{ }^{13} \mathrm{C}\right)$}



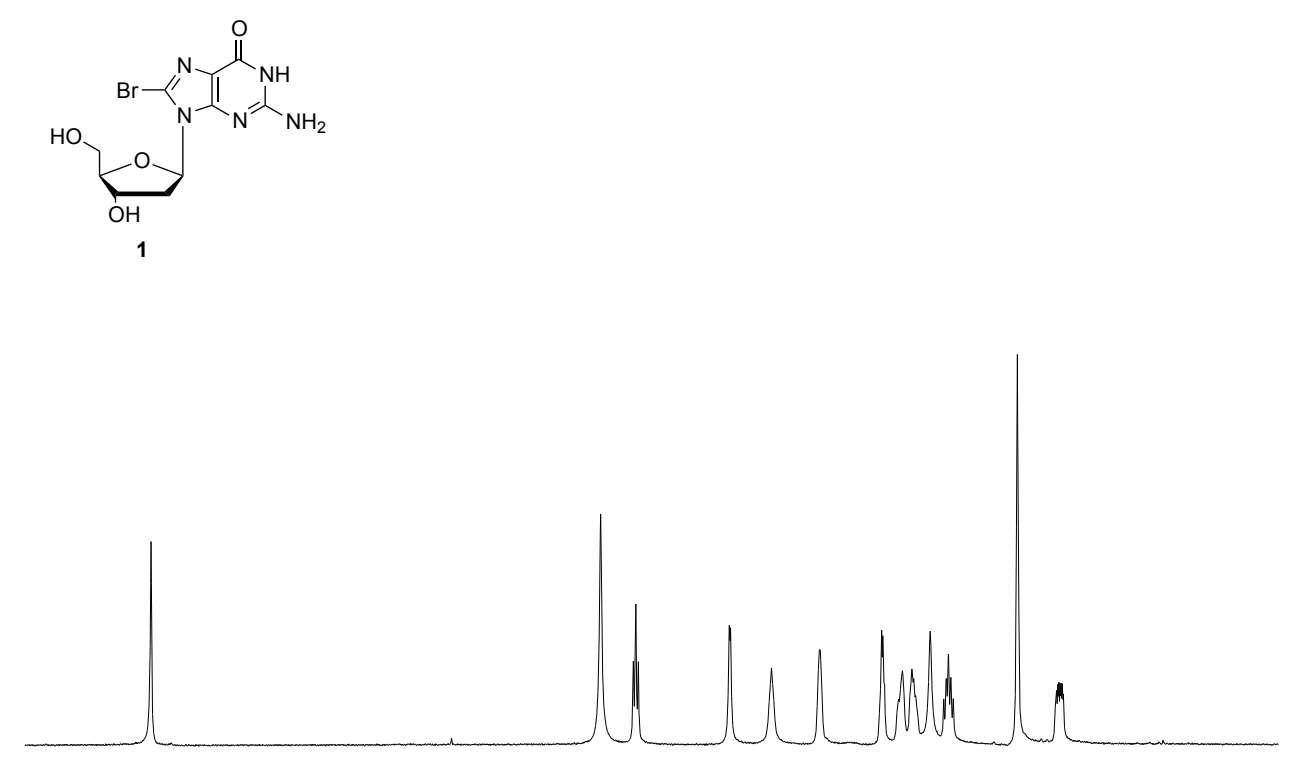

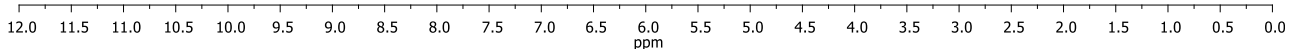

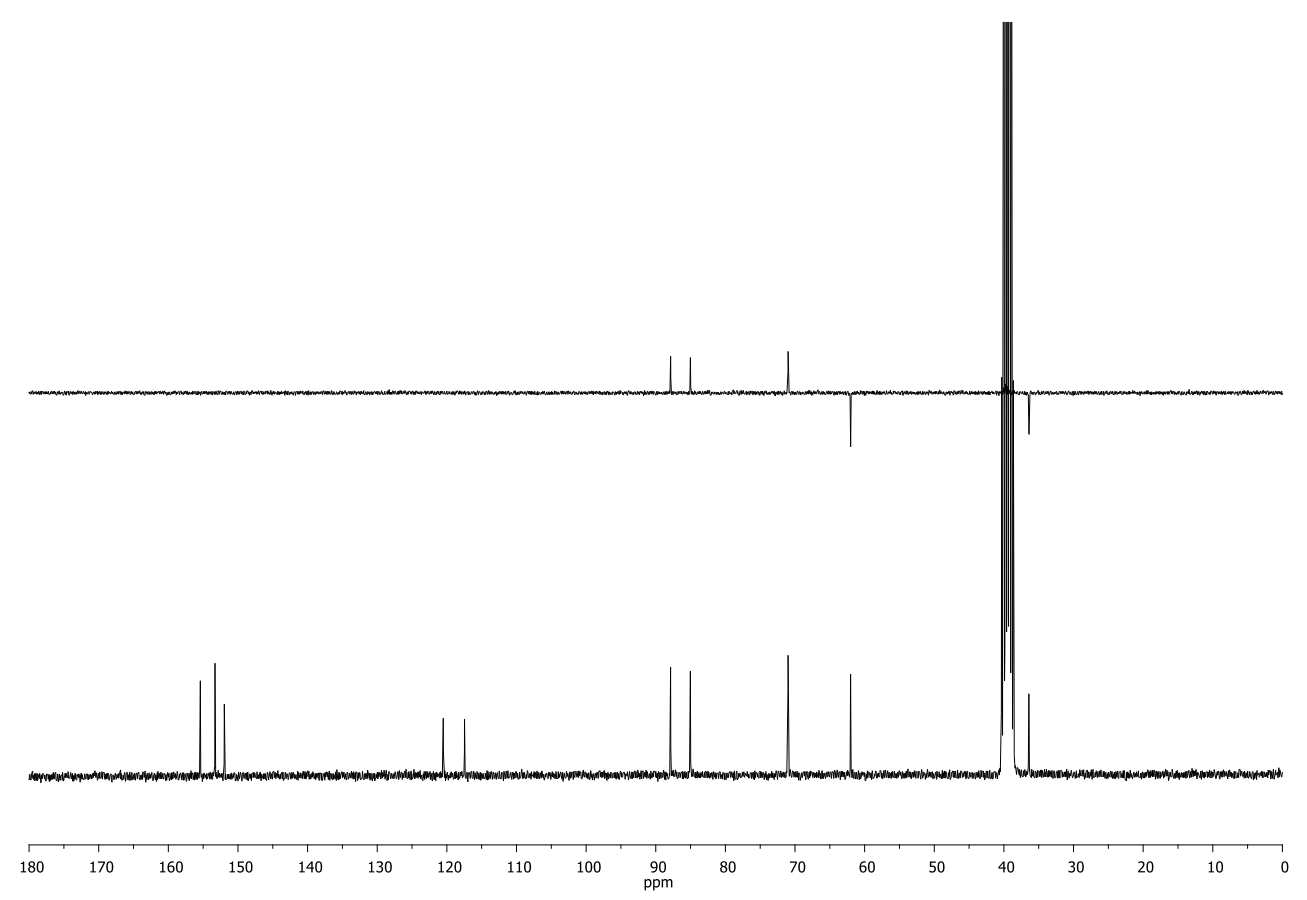



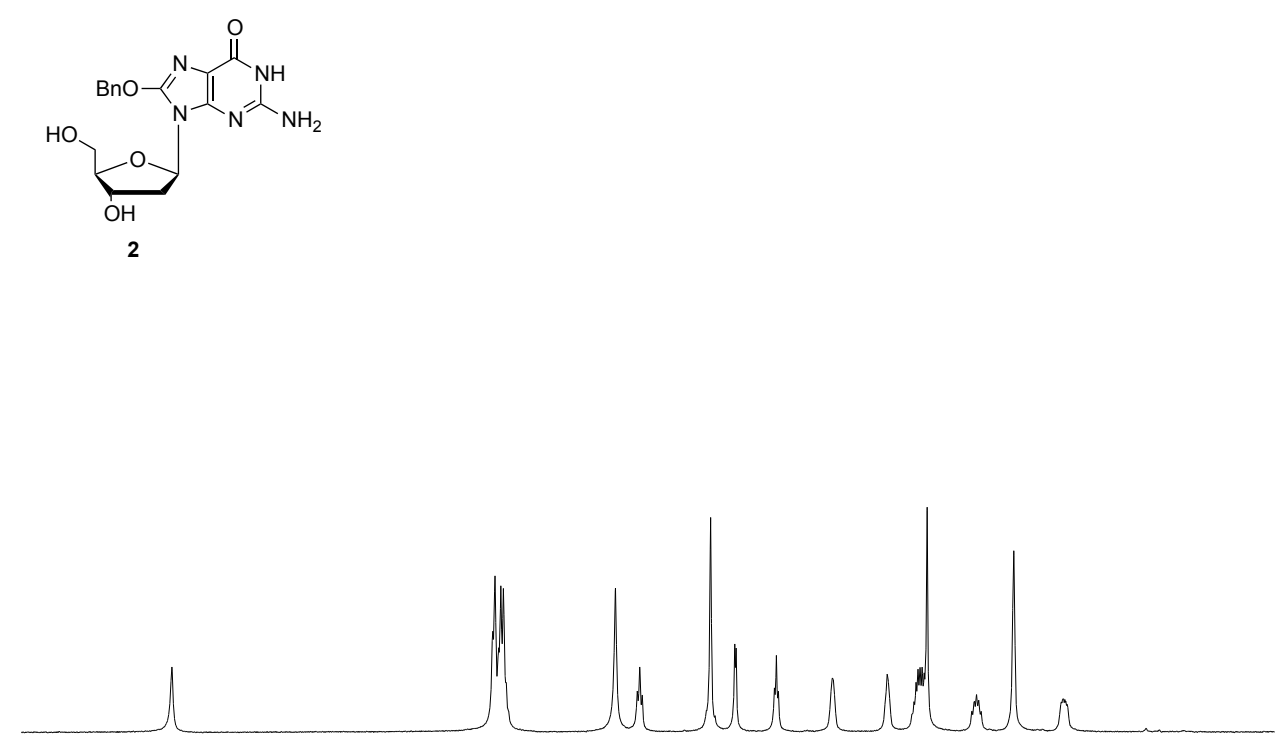

$\begin{array}{rrrrrrrrrrrrrrrrrrrrrrrrrrrrrrrr}12.0 & 11.5 & 11.0 & 10.5 & 10.0 & 9.5 & 9.0 & 8.5 & 8.0 & 7.5 & 7.0 & 6.5 & 6.0 & 5.5 & 5.0 & 4.5 & 4.0 & 3.5 & 3.0 & 2.5 & 2.0 & 1.5 & 1.0 & 0.5 & 0.0\end{array}$

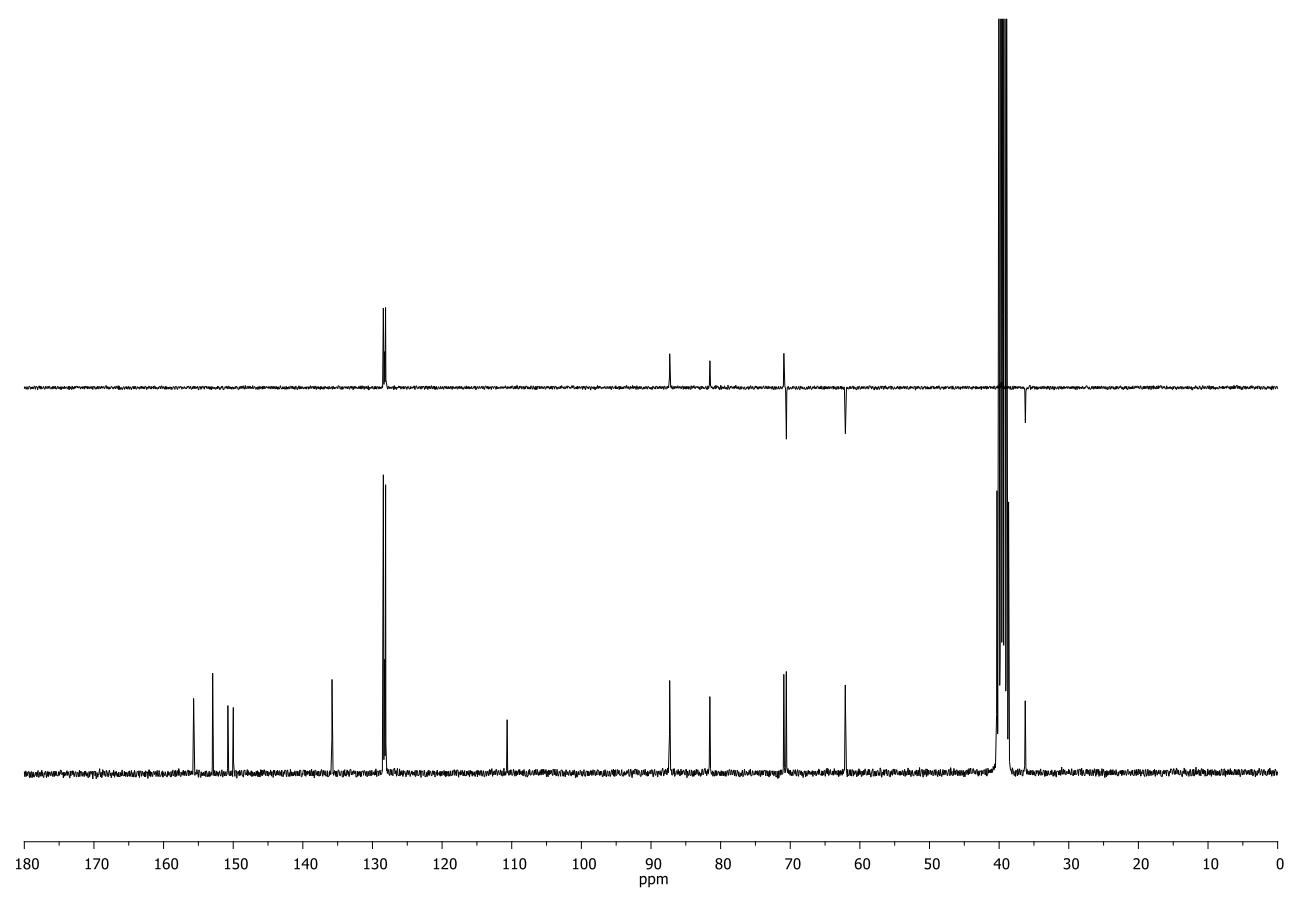



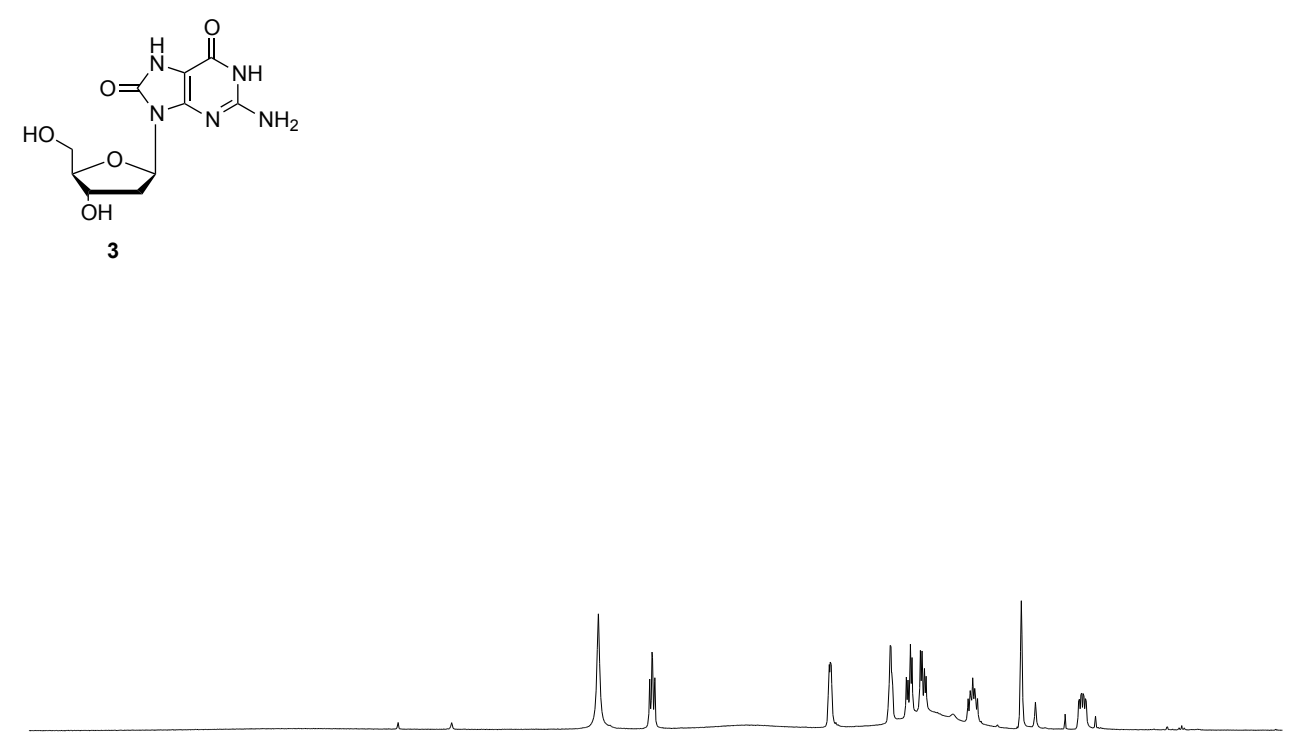

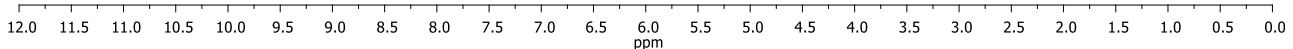

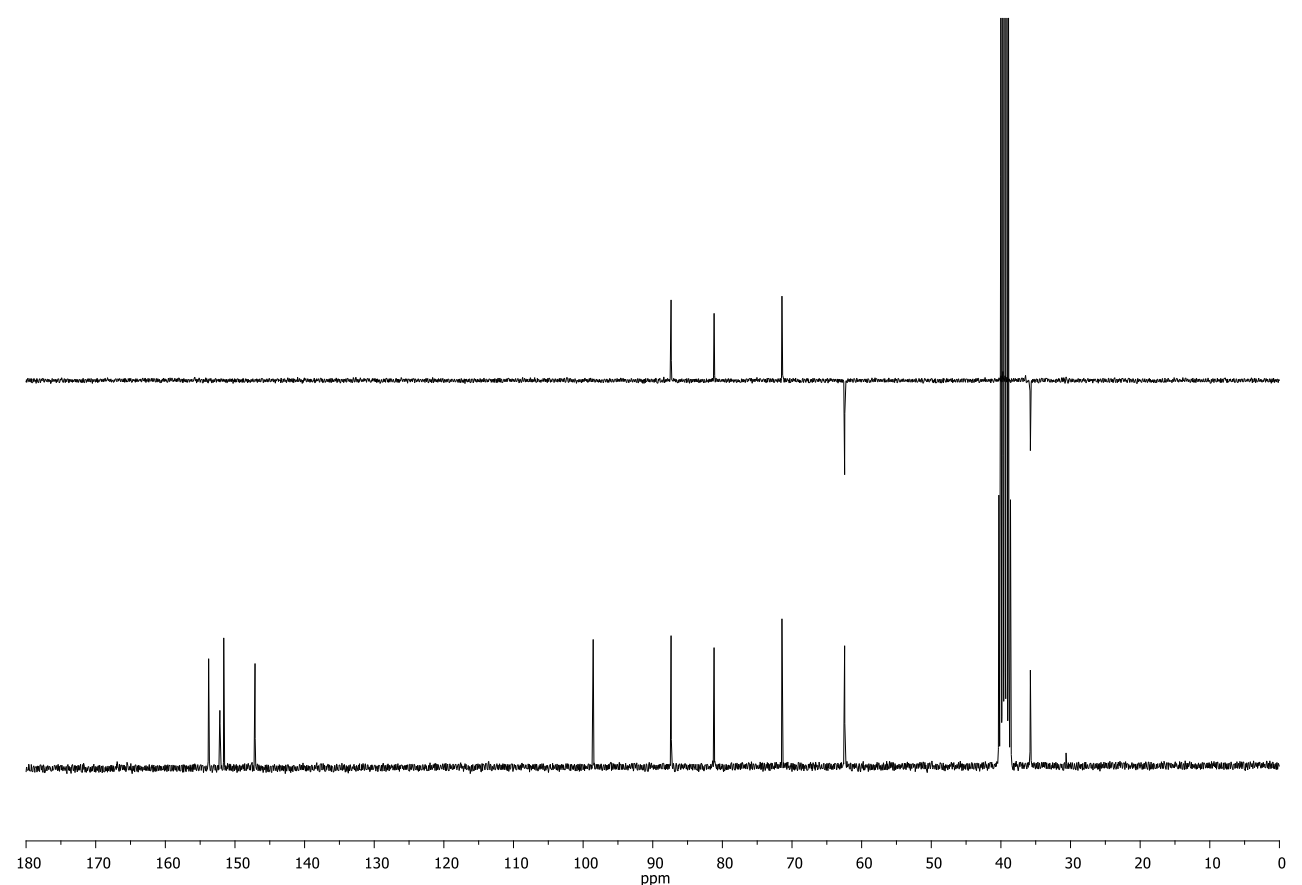



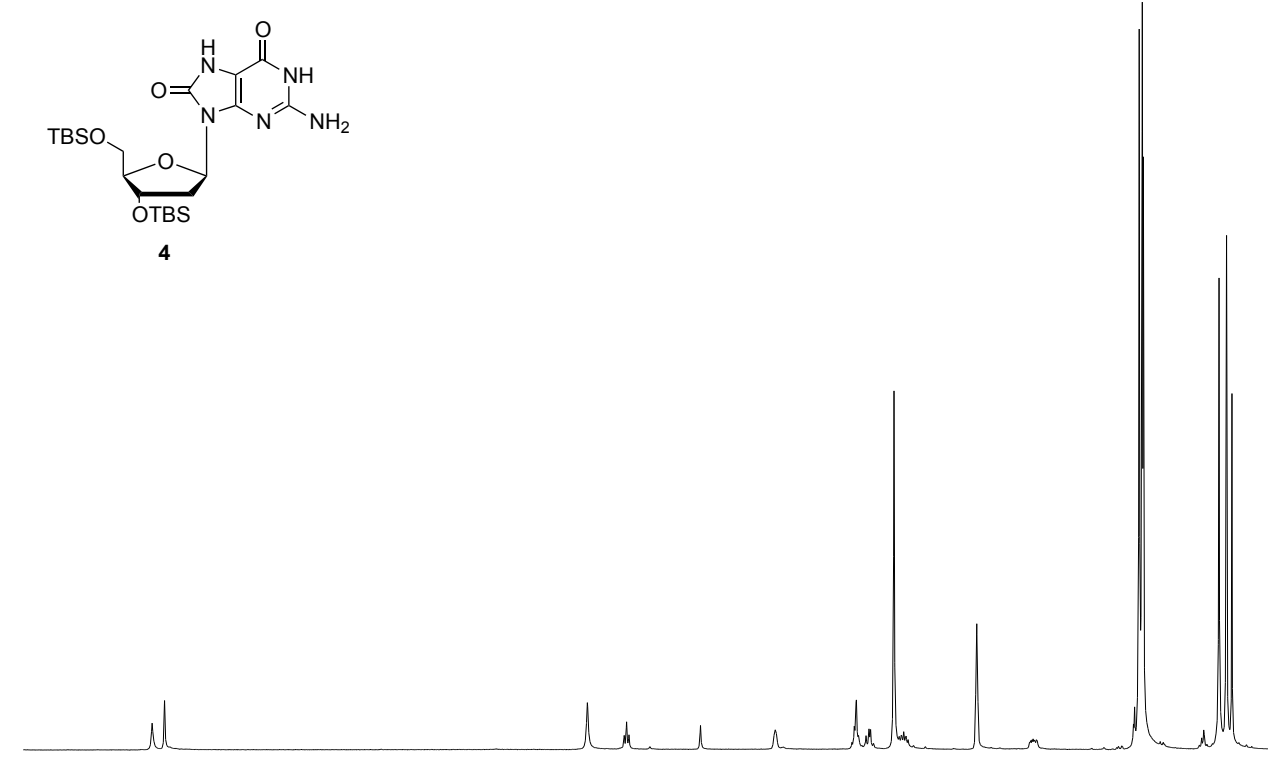

$\begin{array}{lllllllllllllllllllllllllllllll}12.0 & 11.5 & 11.0 & 10.5 & 10.0 & 9.5 & 9.0 & 8.5 & 8.0 & 7.5 & 7.0 & 6.5 & 6.0 & 5.5 & 5.0 & 4.5 & 4.0 & 3.5 & 3.0 & 2.5 & 2.0 & 1.5 & 1.0 & 0.5 & 0.0 & -0.1\end{array}$

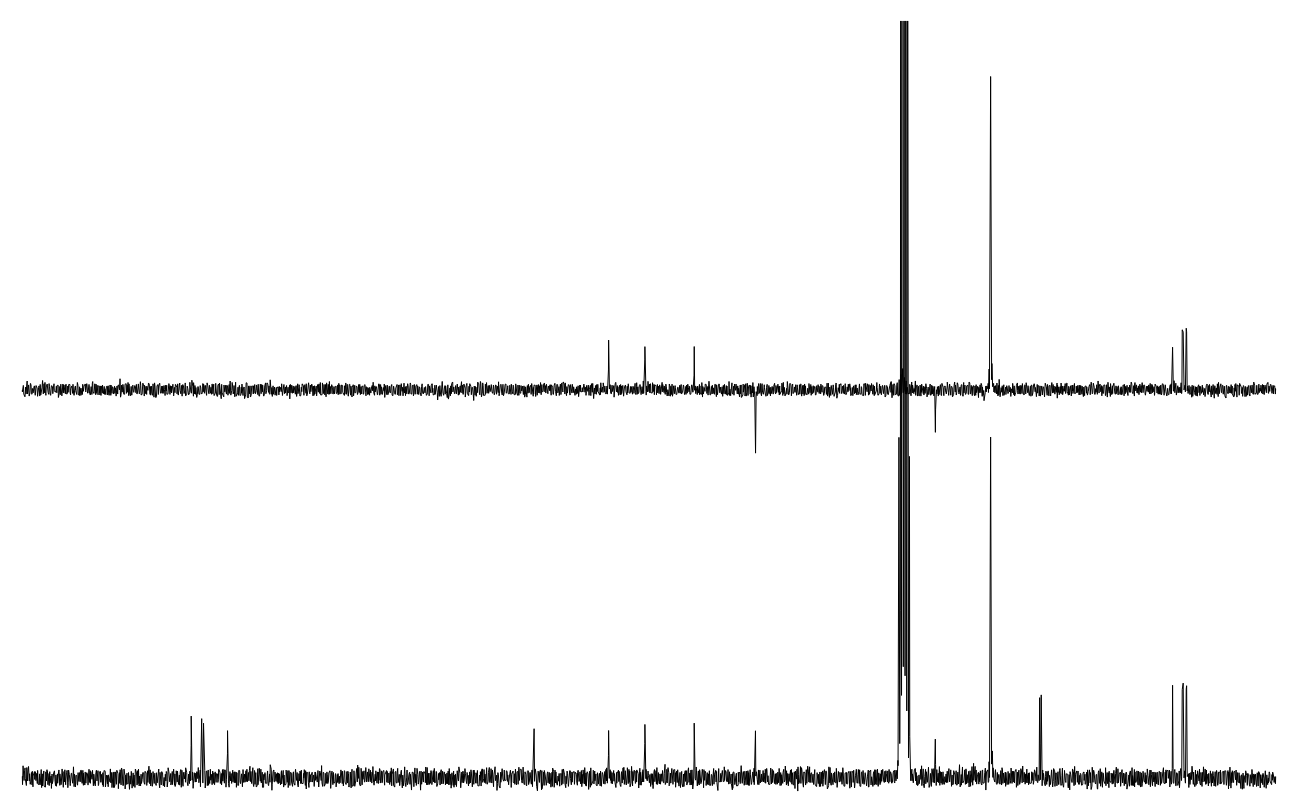

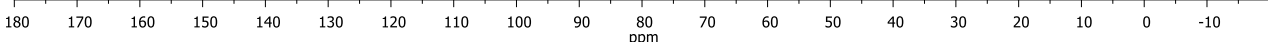



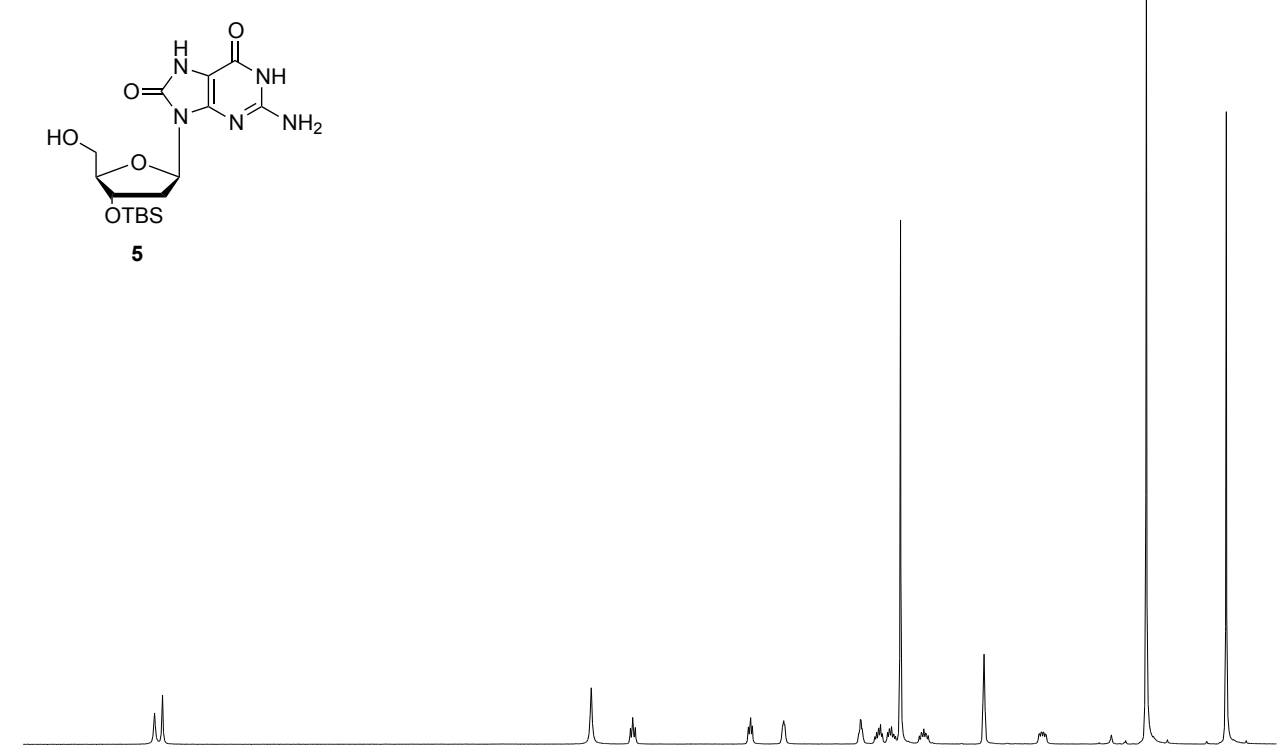

$\begin{array}{lllllllllllllllllllllllllllllllllll}12.0 & 11.5 & 11.0 & 10.5 & 10.0 & 9.5 & 9.0 & 8.5 & 8.0 & 7.5 & 7.0 & 6.5 & 6.0 & 5.5 & 5.0 & 4.5 & 4.0 & 3.5 & 3.0 & 2.5 & 2.0 & 1.5 & 1.0 & 0.5 & 0.0 & -0.5\end{array}$

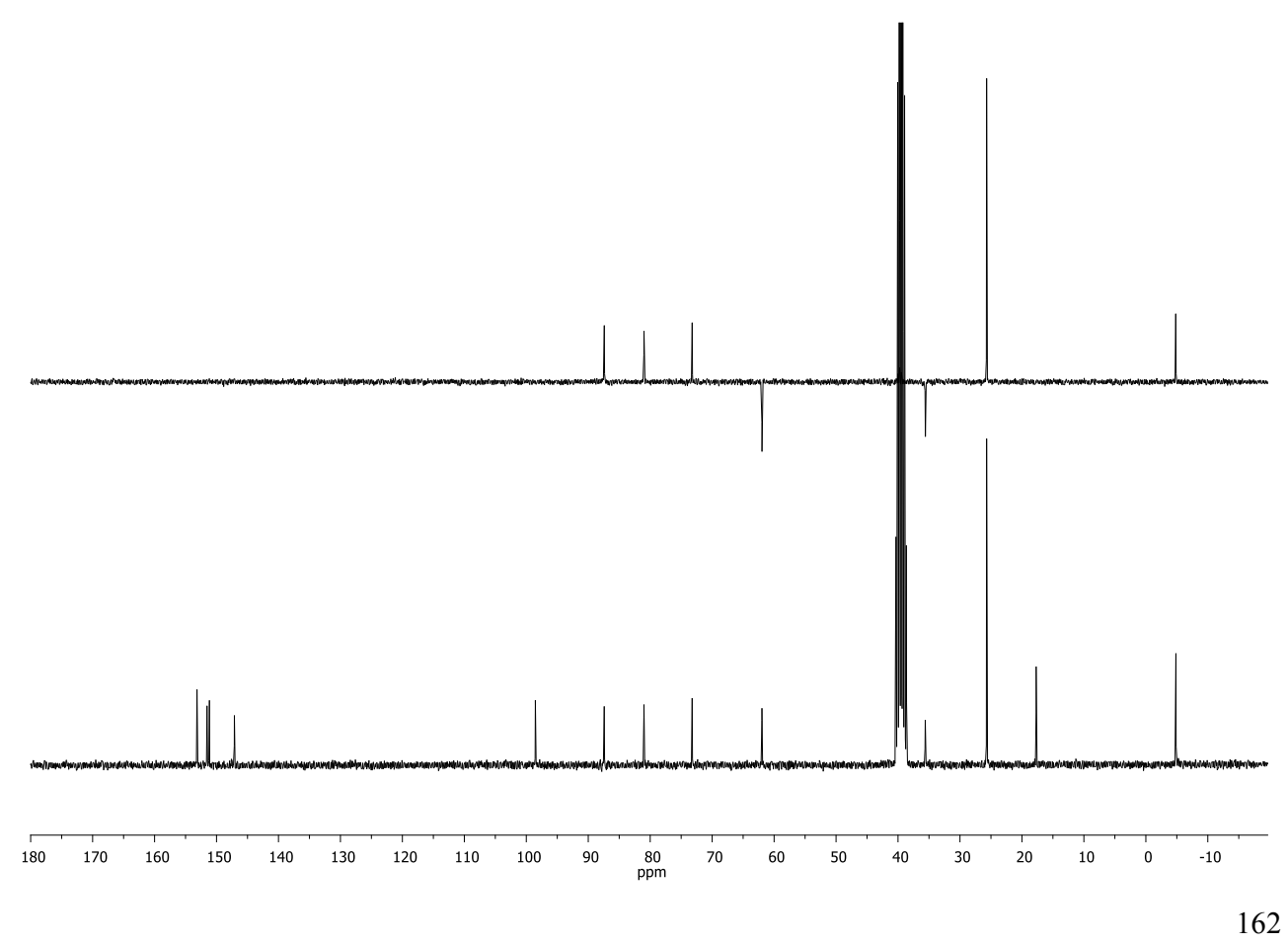



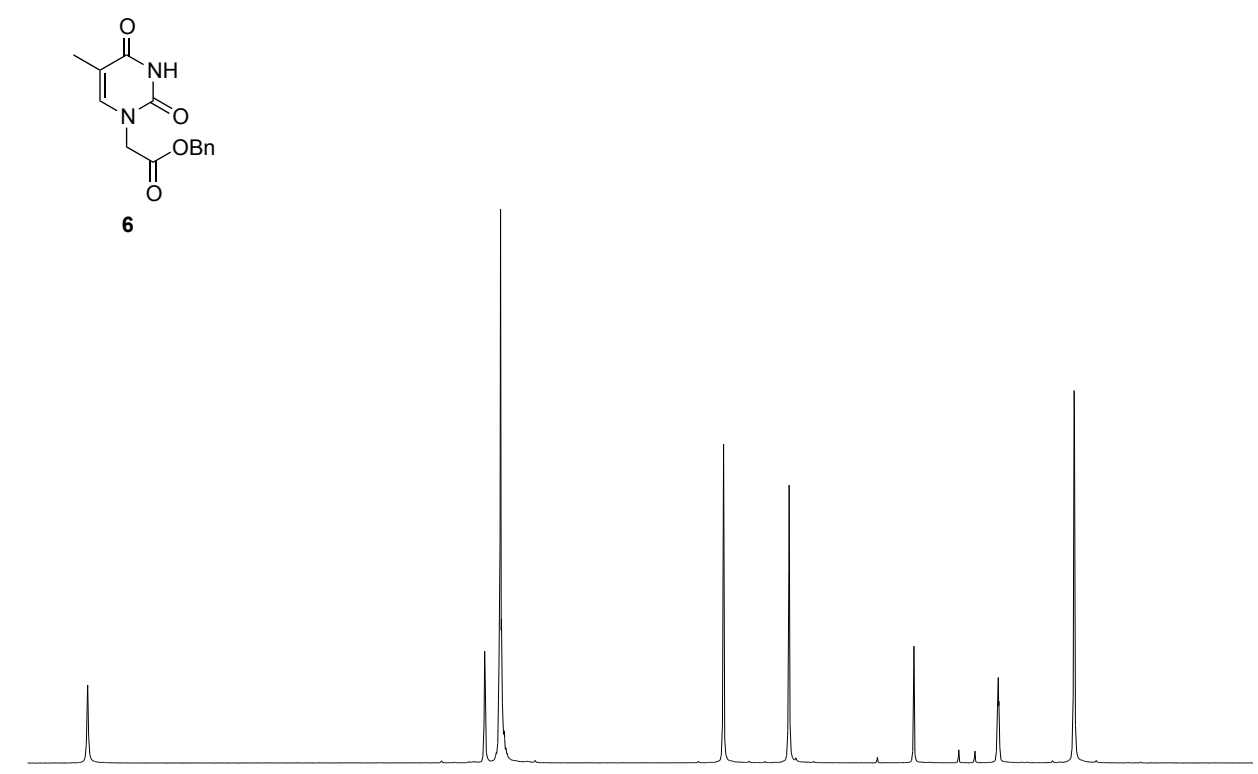

$\begin{array}{rrrrrrrrrrrrrrrrrrrrrrrrrr}12.0 & 11.5 & 11.0 & 10.5 & 10.0 & 9.5 & 9.0 & 8.5 & 8.0 & 7.5 & 7.0 & 6.5 & 6.0 & 5.5 & 5.0 & 4.5 & 4.0 & 3.5 & 3.0 & 2.5 & 2.0 & 1.5 & 1.0 & 0.5 & 0.0\end{array}$
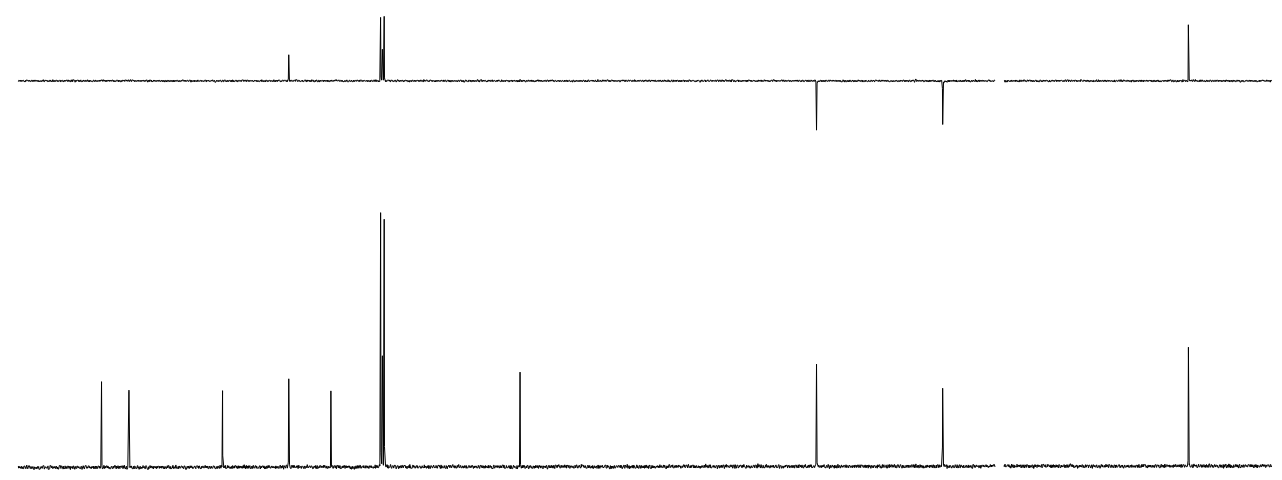

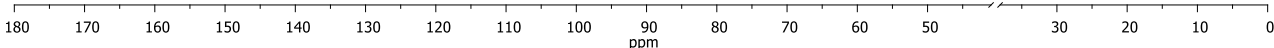



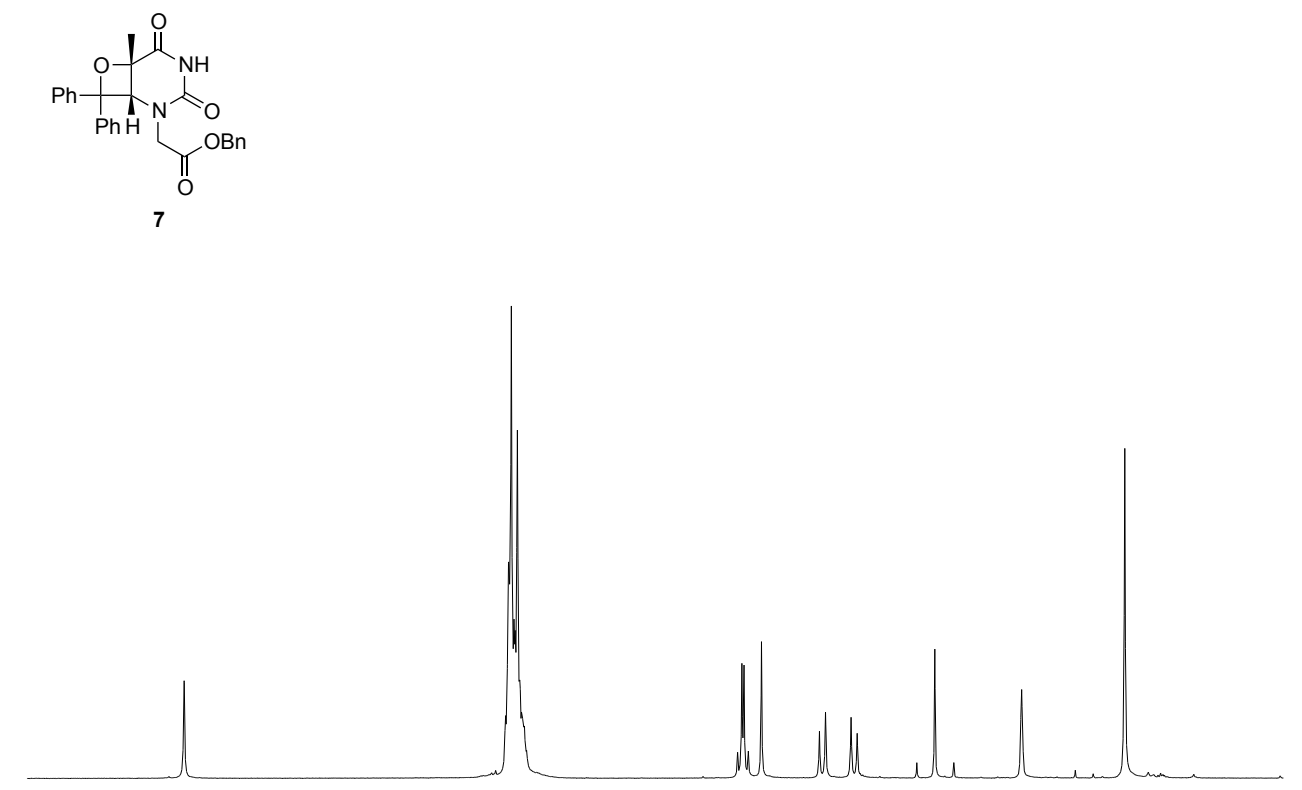

$\begin{array}{llllllllllllllllllllllllllllllll}12.0 & 11.5 & 11.0 & 10.5 & 10.0 & 9.5 & 9.0 & 8.5 & 8.0 & 7.5 & 7.0 & 6.5 & 6.0 & 5.5 & 5.0 & 4.5 & 4.0 & 3.5 & 3.0 & 2.5 & 2.0 & 1.5 & 1.0 & 0.5 & 0.0\end{array}$
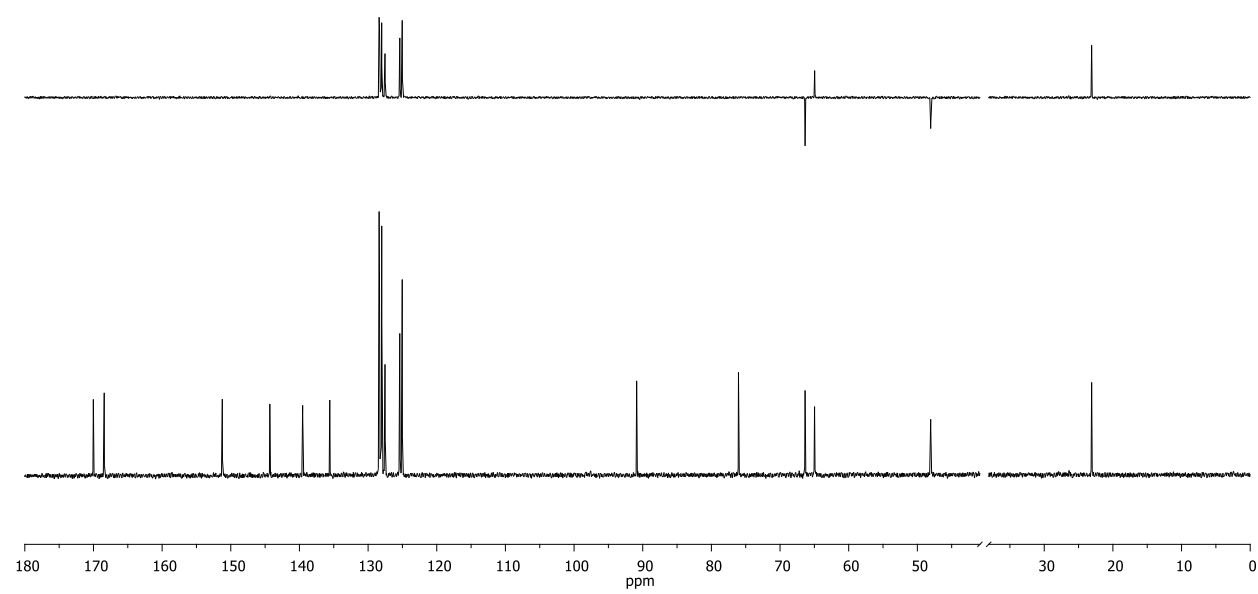


$$
{ }_{8}^{O H}
$$
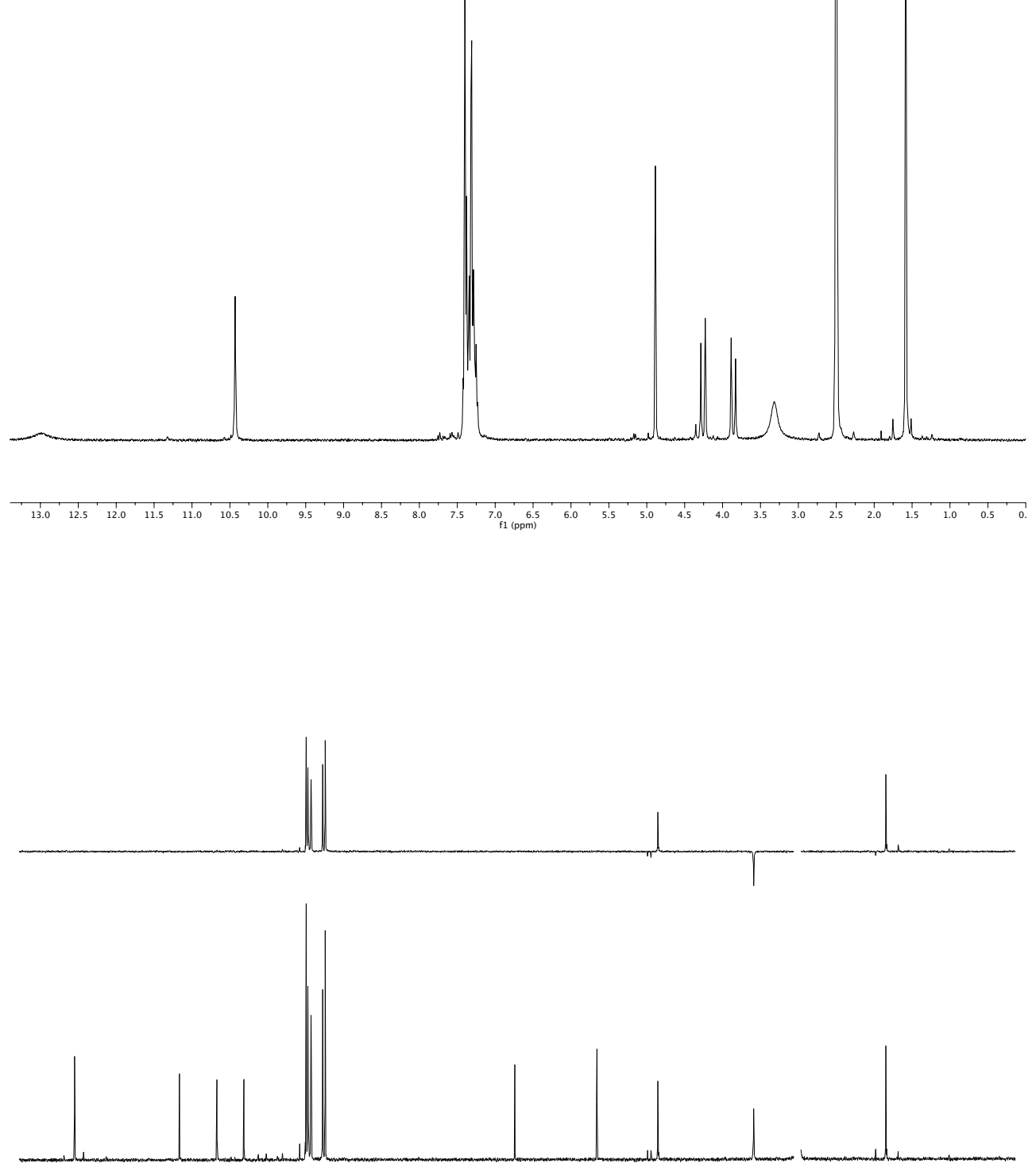

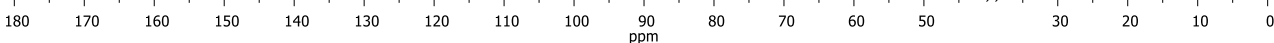



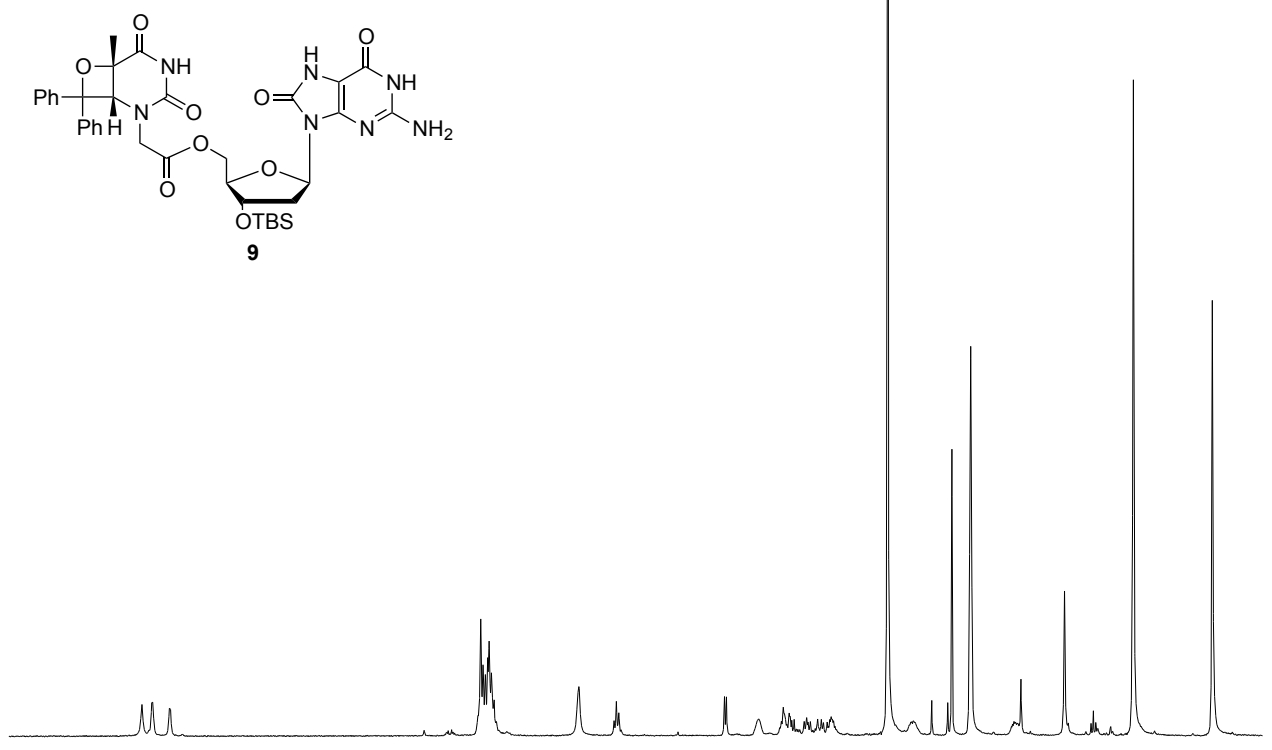

$\begin{array}{rlllllllllllllllllllllllllllllllllll}12.0 & 11.5 & 11.0 & 10.5 & 10.0 & 9.5 & 9.0 & 8.5 & 8.0 & 7.5 & 7.0 & 6.5 & 6.0 & 5.5 & 5.0 & 4.5 & 4.0 & 3.5 & 3.0 & 2.5 & 2.0 & 1.5 & 1.0 & 0.5 & 0.0 & -0.1\end{array}$

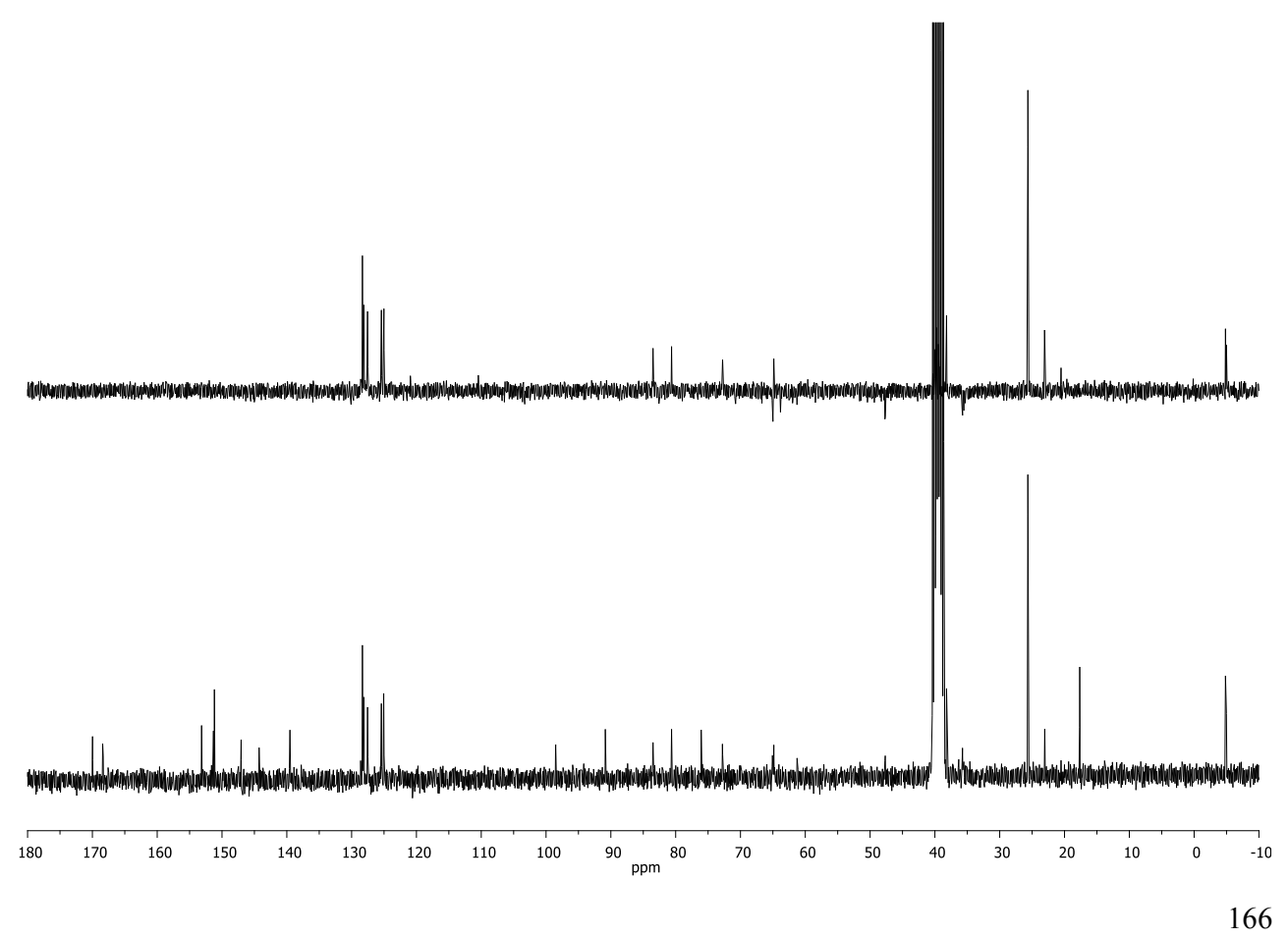



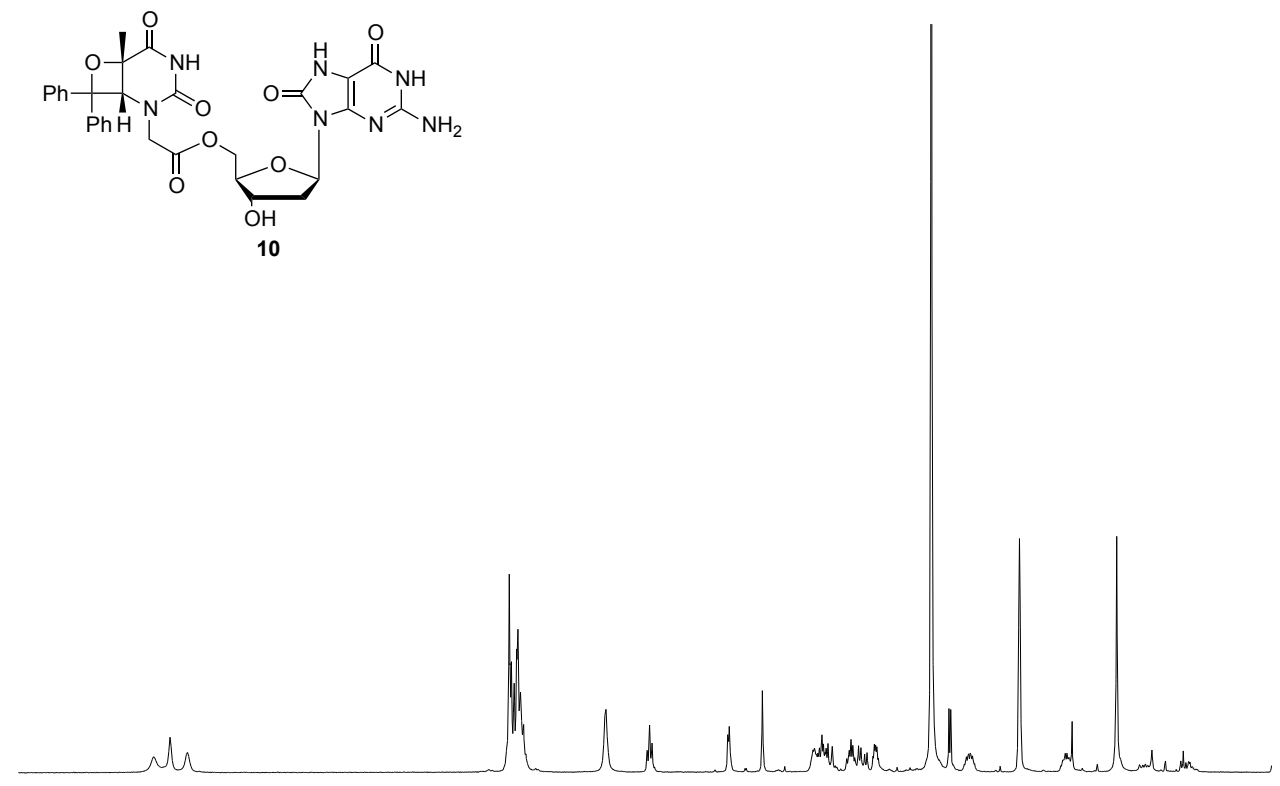

$\begin{array}{llllllllllllllllllllllllllllll}12.0 & 11.5 & 11.0 & 10.5 & 10.0 & 9.5 & 9.0 & 8.5 & 8.0 & 7.5 & 7.0 & 6.5 & 6.0 & 5.5 & 5.0 & 4.5 & 4.0 & 3.5 & 3.0 & 2.5 & 2.0 & 1.5 & 1.0 & 0.5 & 0.0\end{array}$
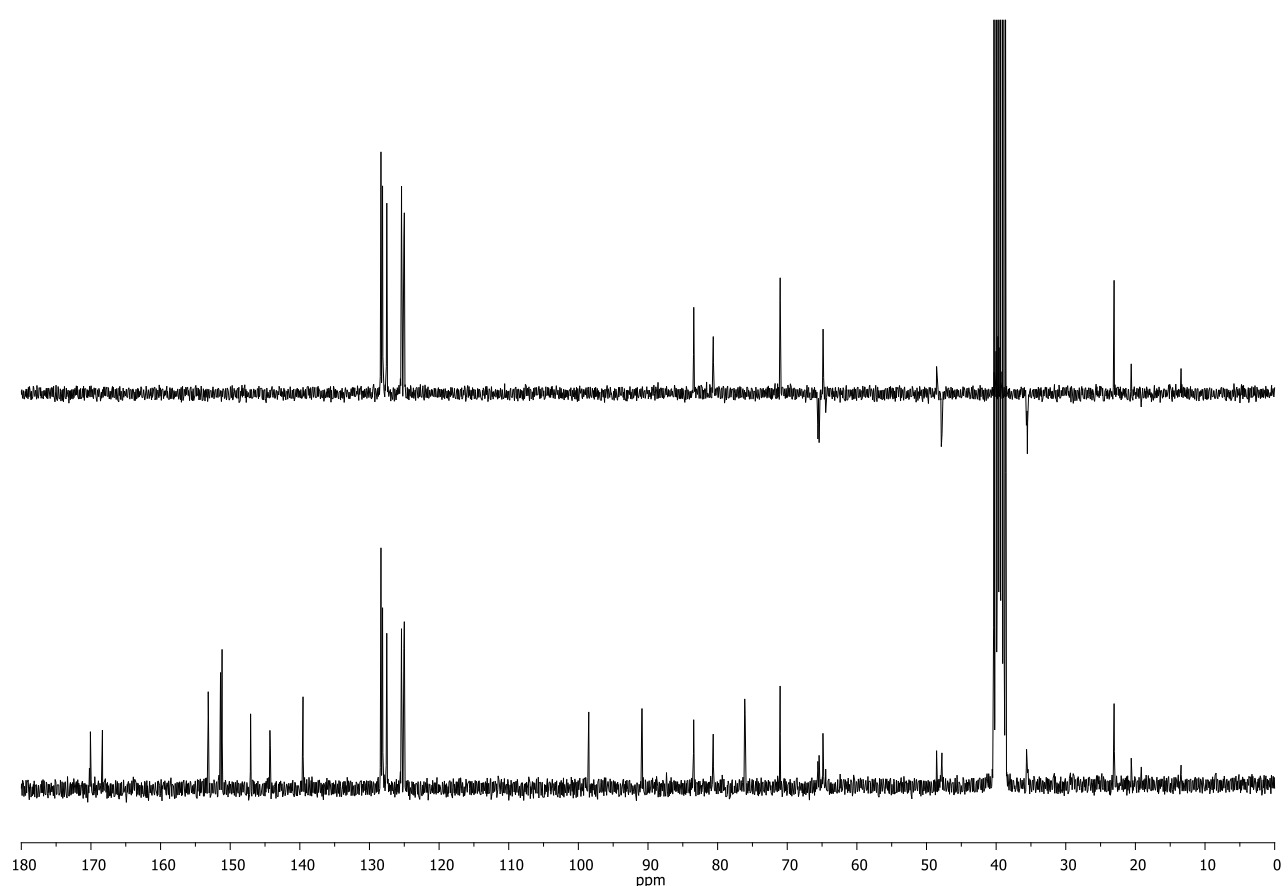


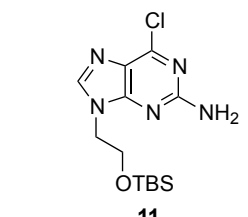

11

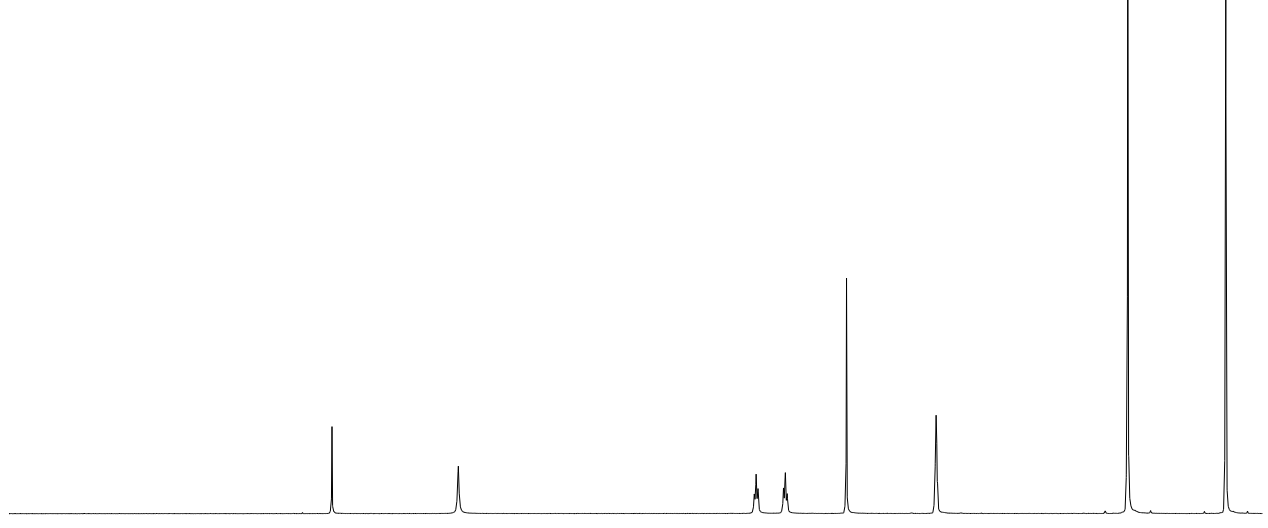

$\begin{array}{lllllllllllllllllllllllllllllll}11.0 & 10.5 & 10.0 & 9.5 & 9.0 & 8.5 & 8.0 & 7.5 & 7.0 & 6.5 & 6.0 & 5.5 & 5.0 & 4.5 & 4.0 & 3.5 & 3.0 & 2.5 & 2.0 & 1.5 & 1.0 & 0.5 & 0.0 & \end{array}$
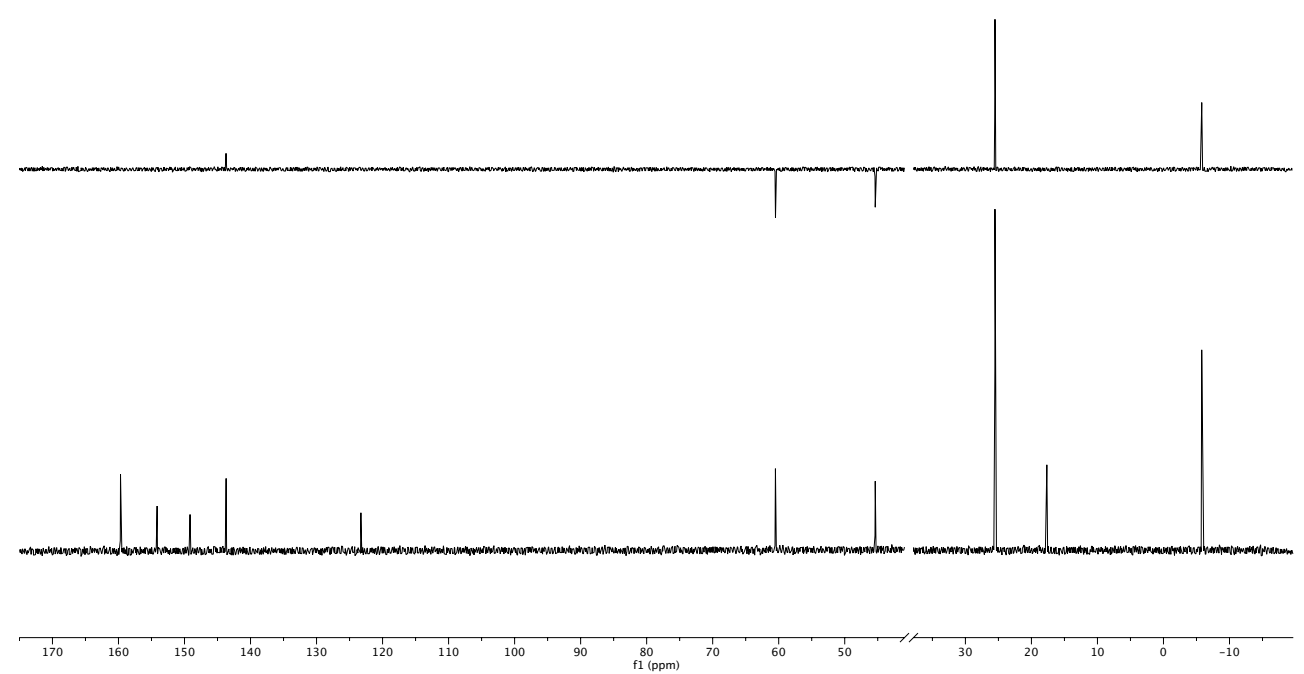


$$
\mathrm{OH}_{12}^{\mathrm{OHH}}
$$

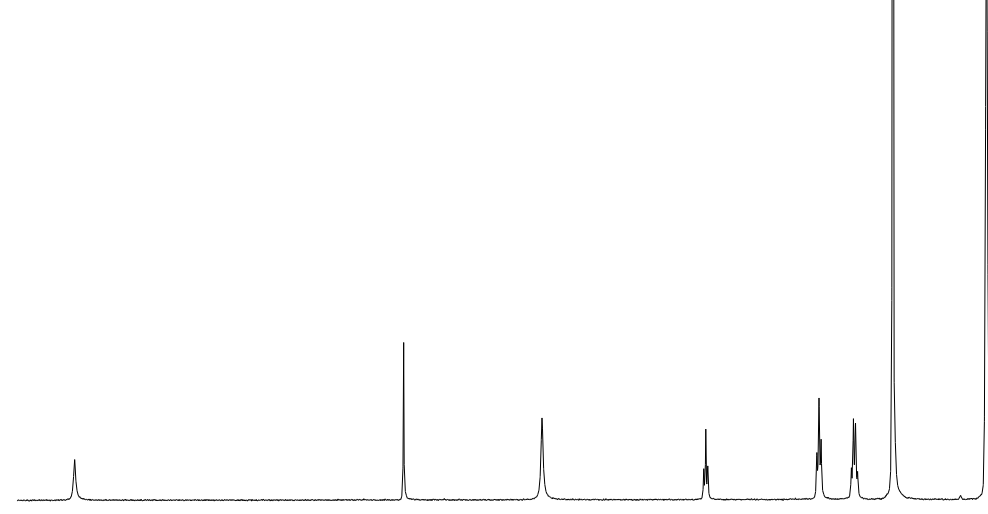

\begin{tabular}{rccccccccccccccccccccccccc}
\hline 1.0 & 10.5 & 10.0 & 9.5 & 9.0 & 8.5 & 8.0 & 7.5 & 7.0 & 6.5 & 6.0 & 5.5 & 5.0 & 4.5 & 4.0 & 3.5 & 3.0 & 2.5 & 2.0 & 1.5 & 1.0 & 0.5 & 0.0
\end{tabular}
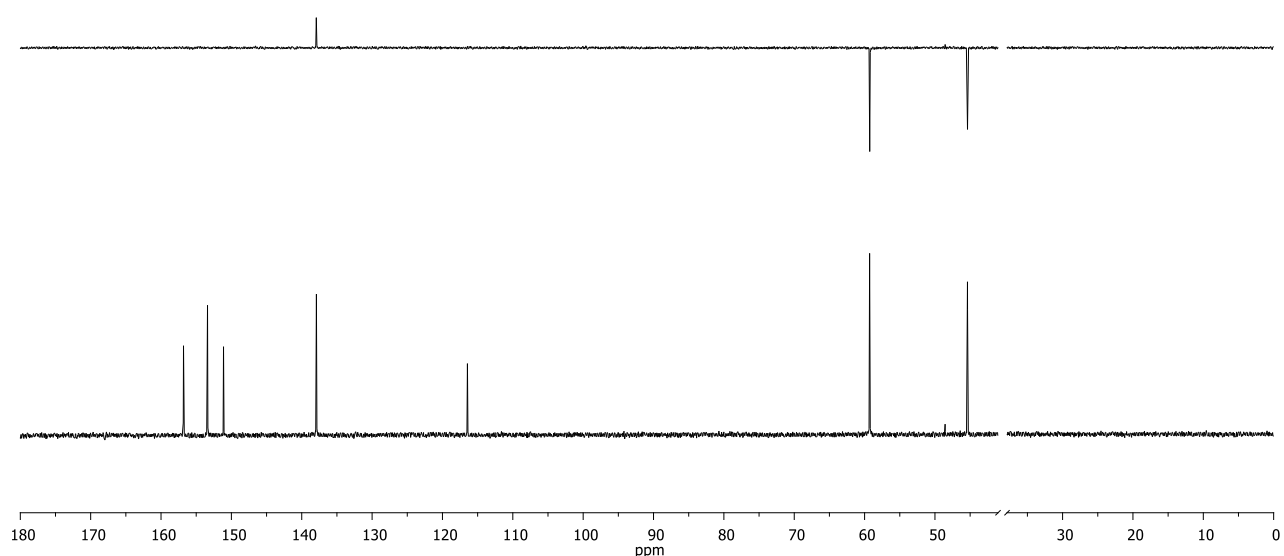

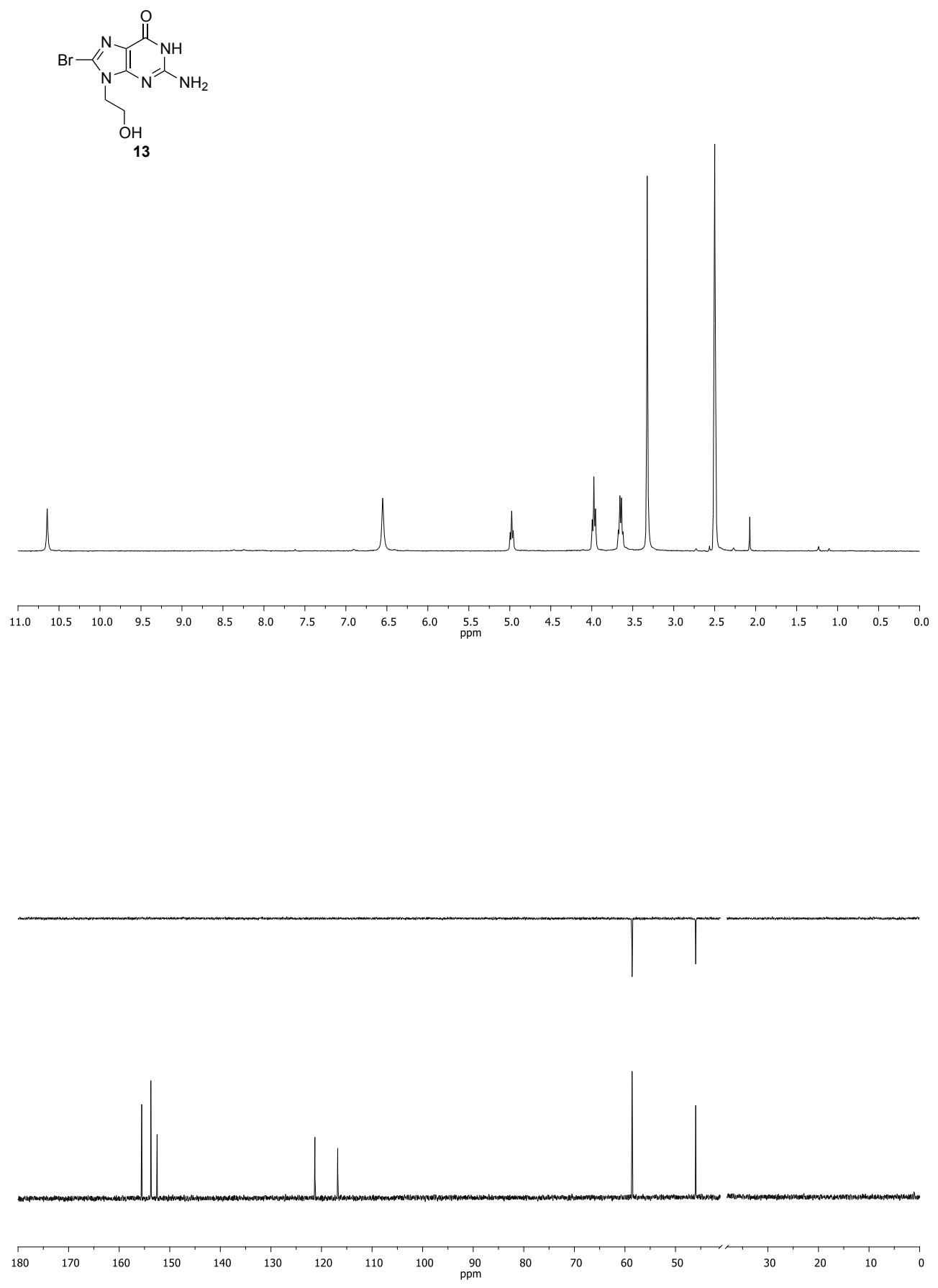

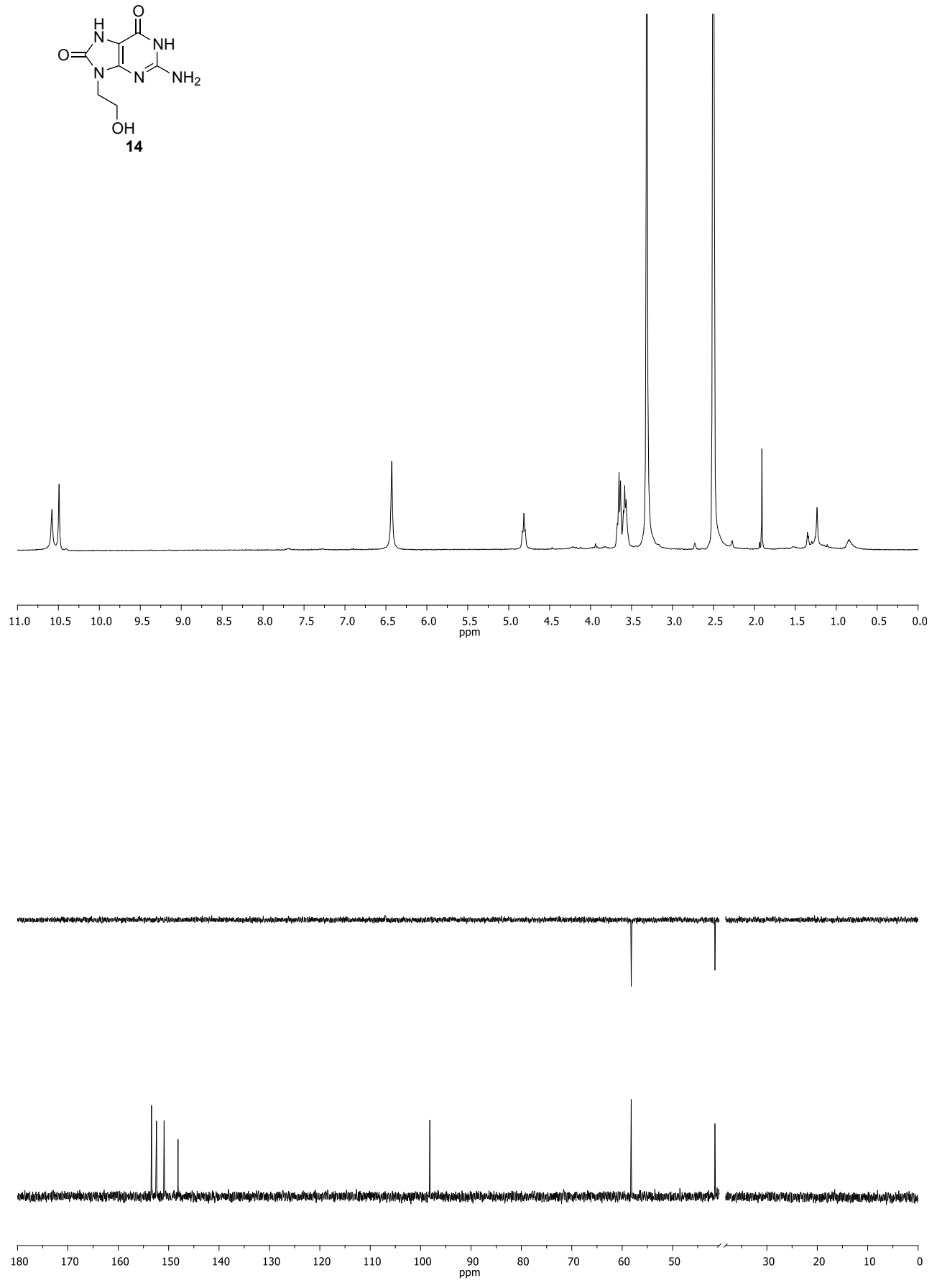

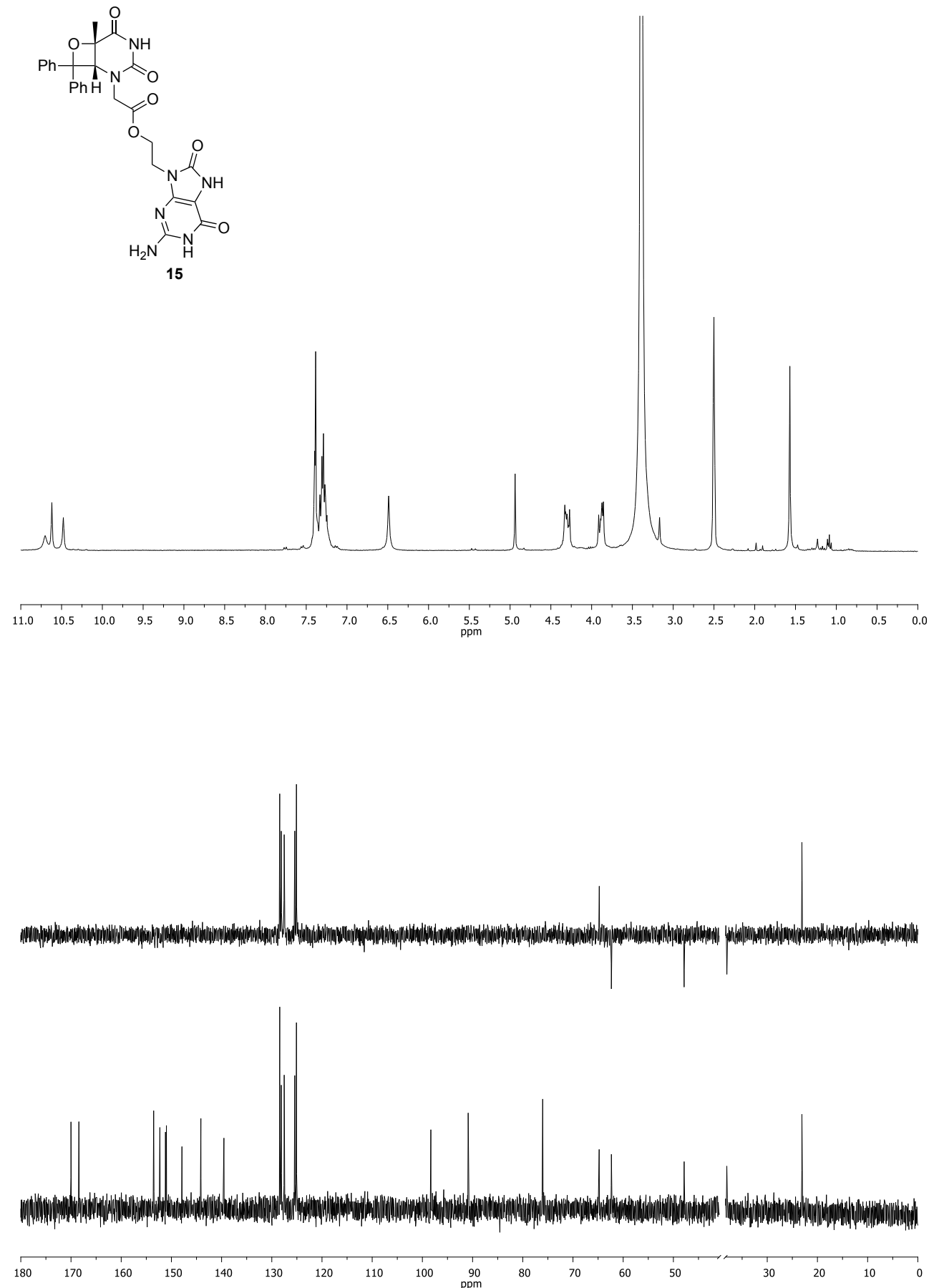

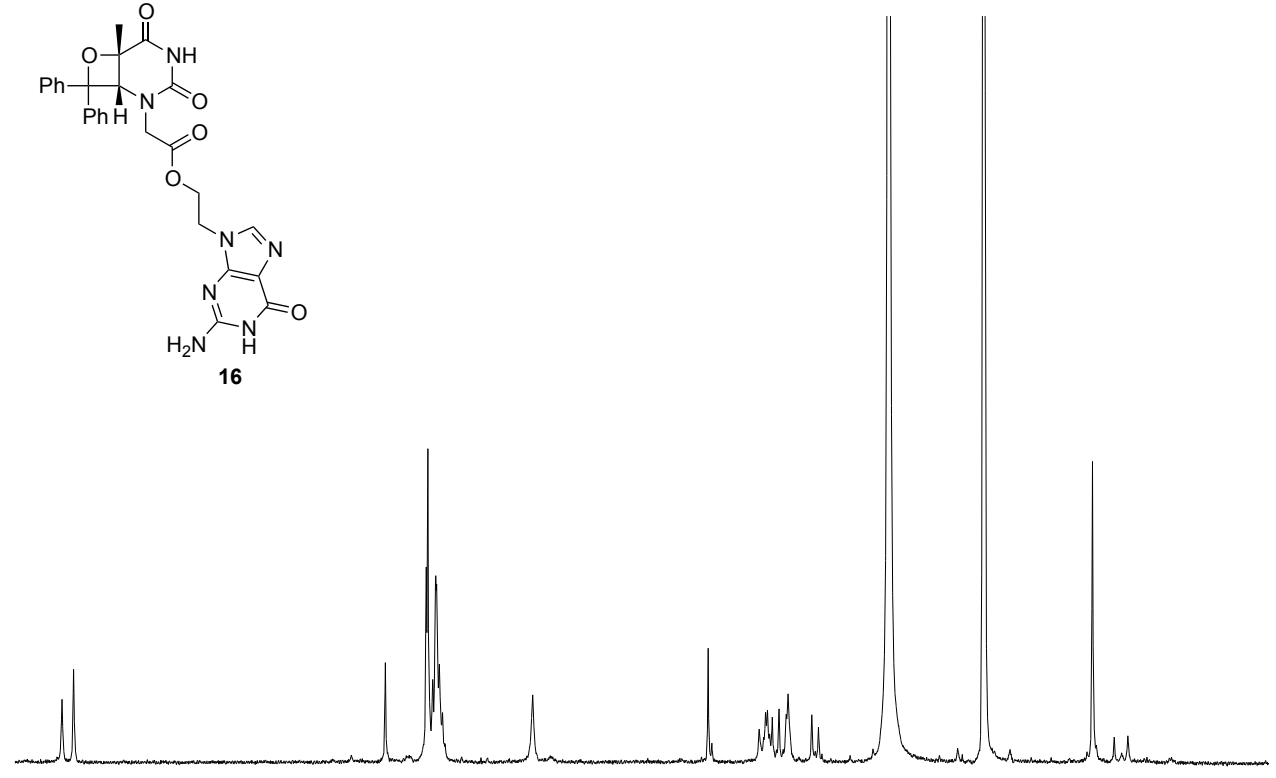

$\begin{array}{lllllllllllllllllllllll}11.0 & 10.5 & 10.0 & 9.5 & 9.0 & 8.5 & 8.0 & 7.5 & 7.0 & 6.5 & 6.0 & \begin{array}{c}5.5 \\ \mathrm{ppm}\end{array} & 5.0 & 4.5 & 4.0 & 3.5 & 3.0 & 2.5 & 2.0 & 1.5 & 1.0 & 0.5 & 0.6\end{array}$
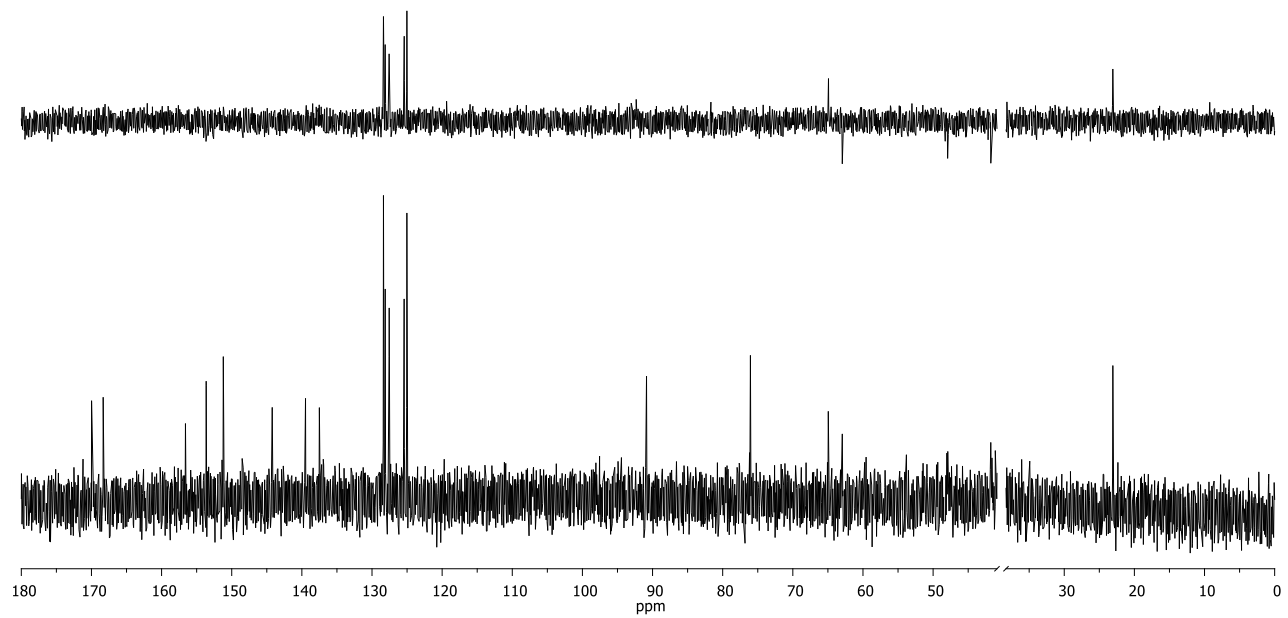

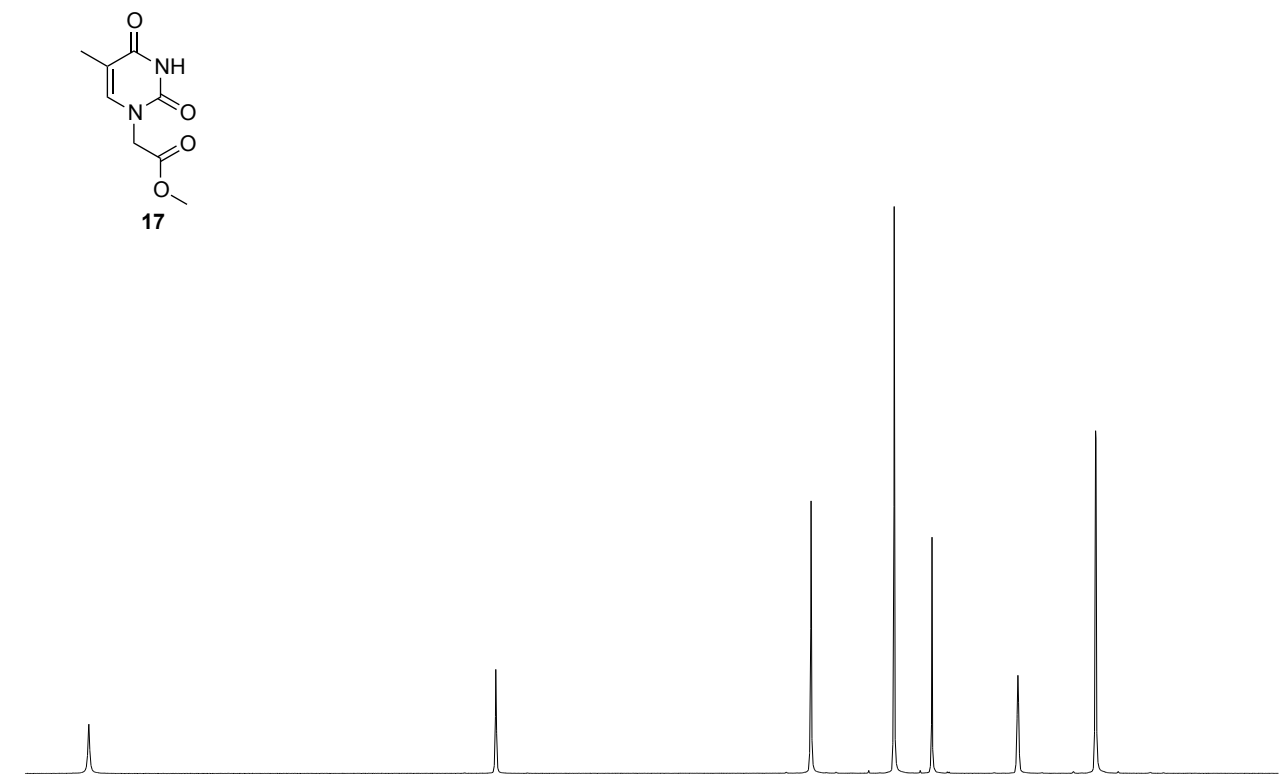

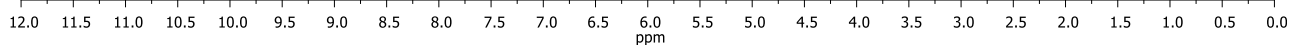
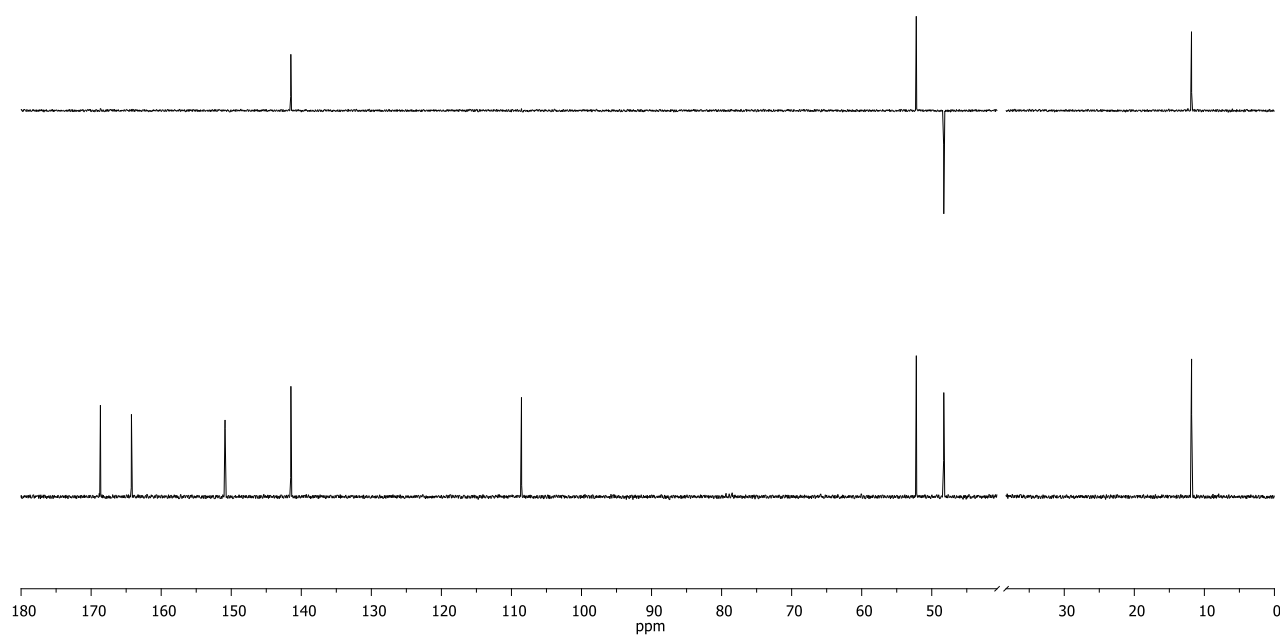

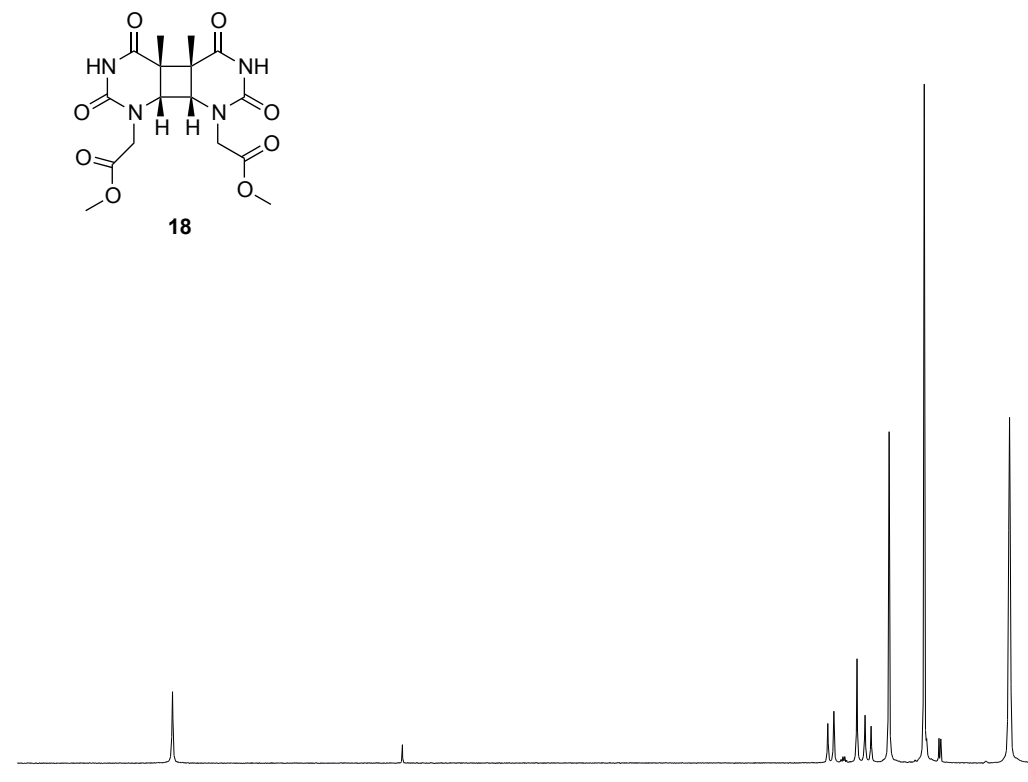

$\begin{array}{rllllllllllllllllllllllllllllll}12.0 & 11.5 & 11.0 & 10.5 & 10.0 & 9.5 & 9.0 & 8.5 & 8.0 & 7.5 & 7.0 & 6.5 & 6.0 & 5.5 & 5.0 & 4.5 & 4.0 & 3.5 & 3.0 & 2.5 & 2.0 & 1.5 & 1.0 & 0.5 & 0.0\end{array}$
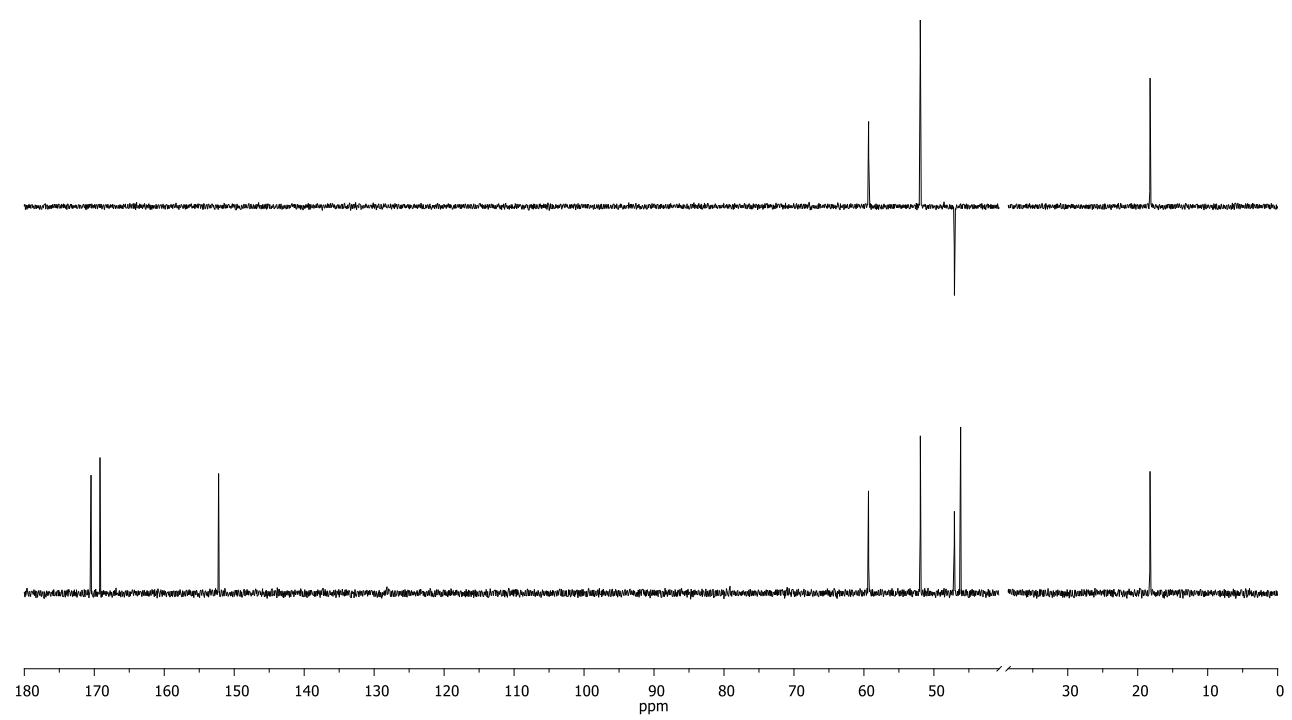

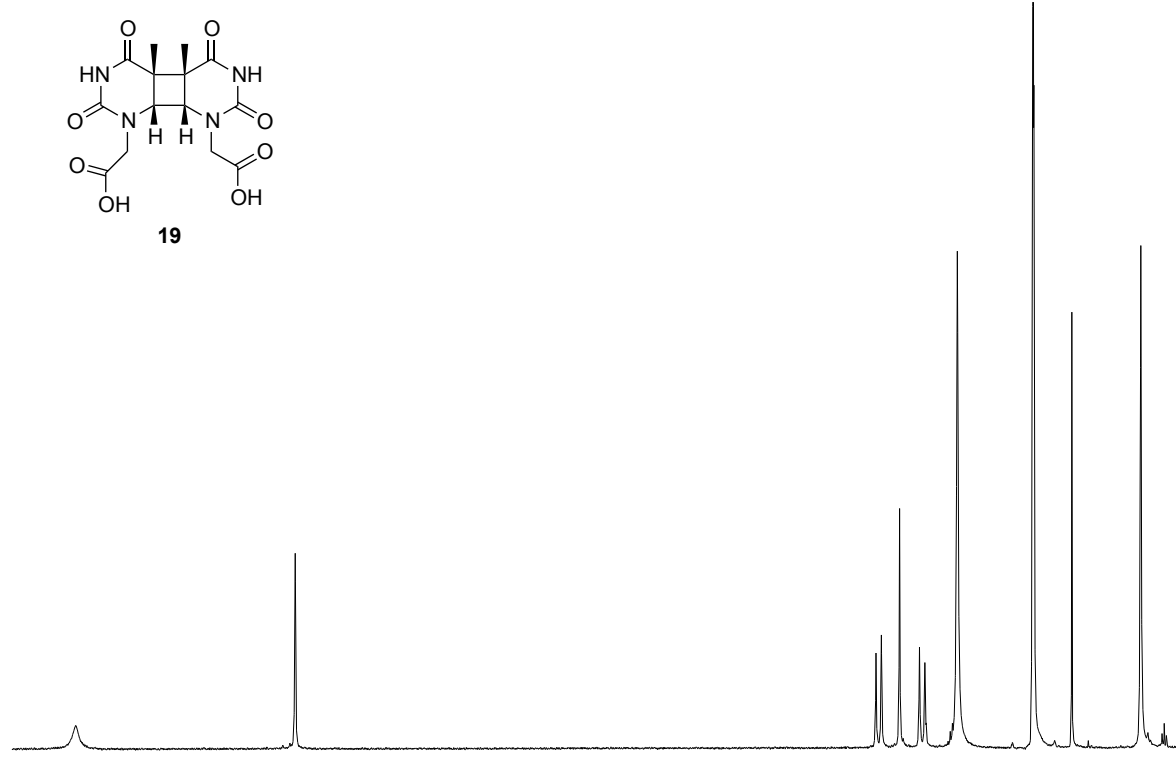

$\begin{array}{lllllllllllllllllllllllllllll}13.5 & 13.0 & 12.5 & 12.0 & 11.5 & 11.0 & 10.5 & 10.0 & 9.5 & 9.0 & 8.5 & 8.0 & 7.5 & 7.0 & 6.5 & 6.0 & 5.5 & 5.0 & 4.5 & 4.0 & 3.5 & 3.0 & 2.5 & 2.0 & 1.5 & 1.0 & 0.5 & 0.0\end{array}$
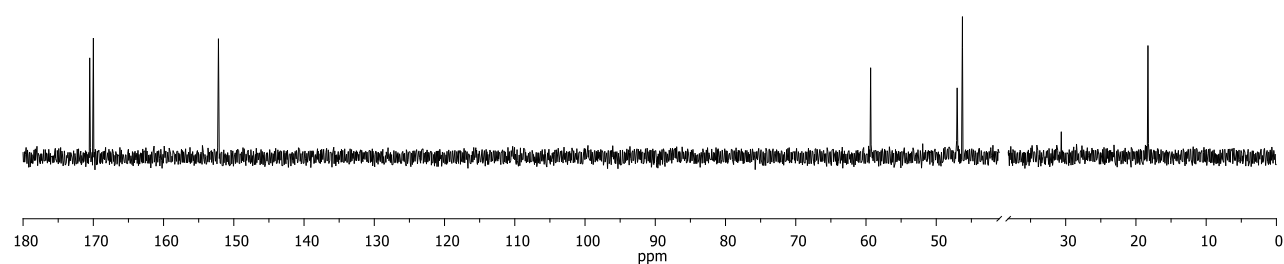

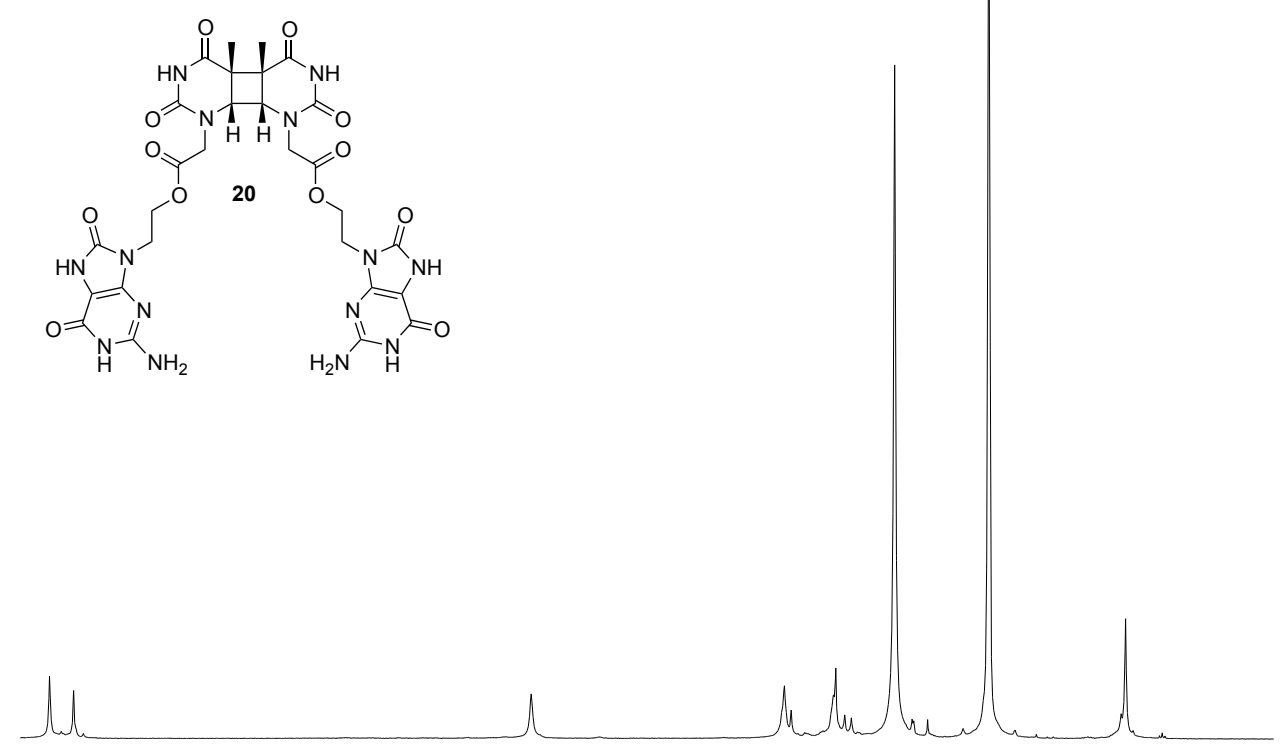

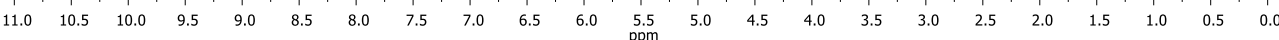
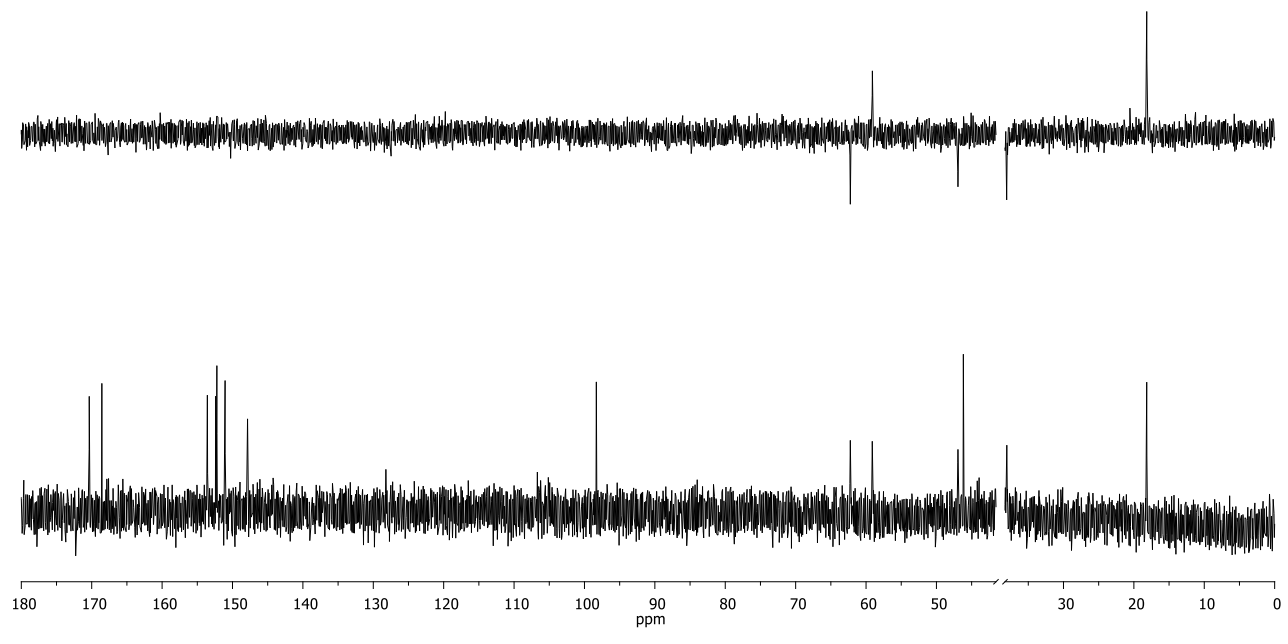

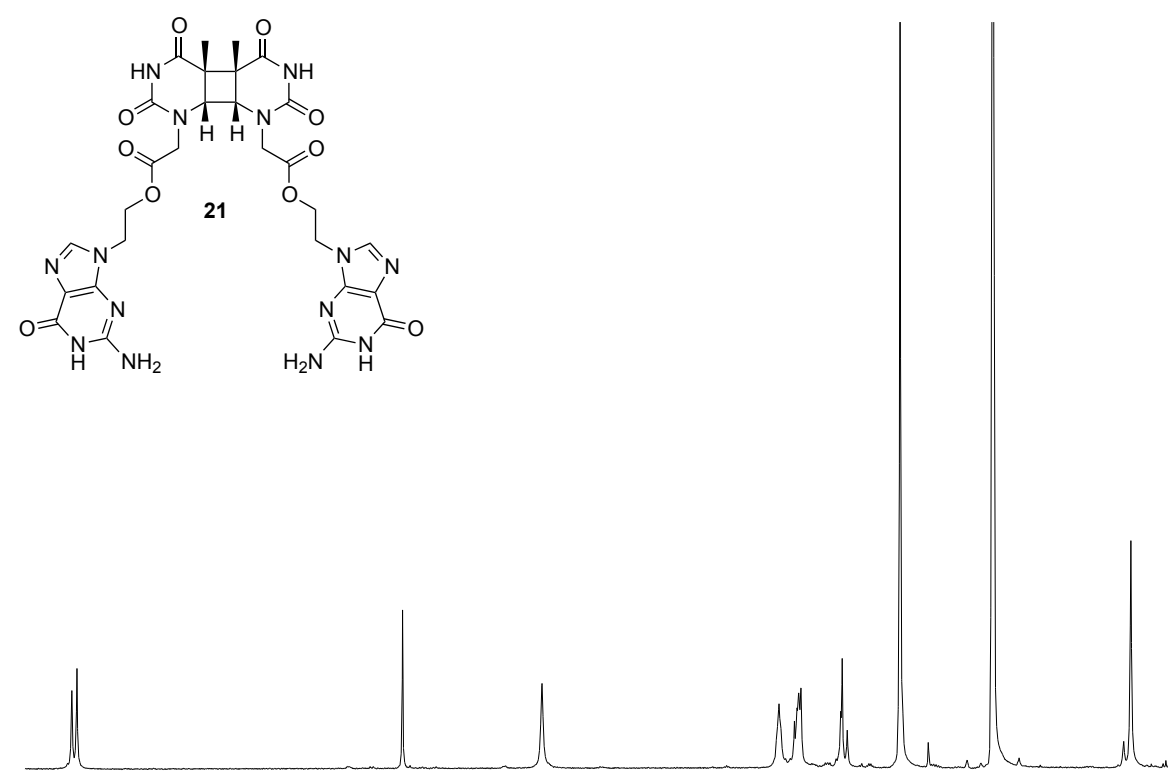

$\begin{array}{llllllllllllllllllllllll}11.0 & 10.5 & 10.0 & 9.5 & 9.0 & 8.5 & 8.0 & 7.5 & 7.0 & 6.5 & 6.0 & 5.5 & 5.0 & 4.5 & 4.0 & 3.5 & 3.0 & 2.5 & 2.0 & 1.5 & 1.0 & 0.5 & 0.0\end{array}$
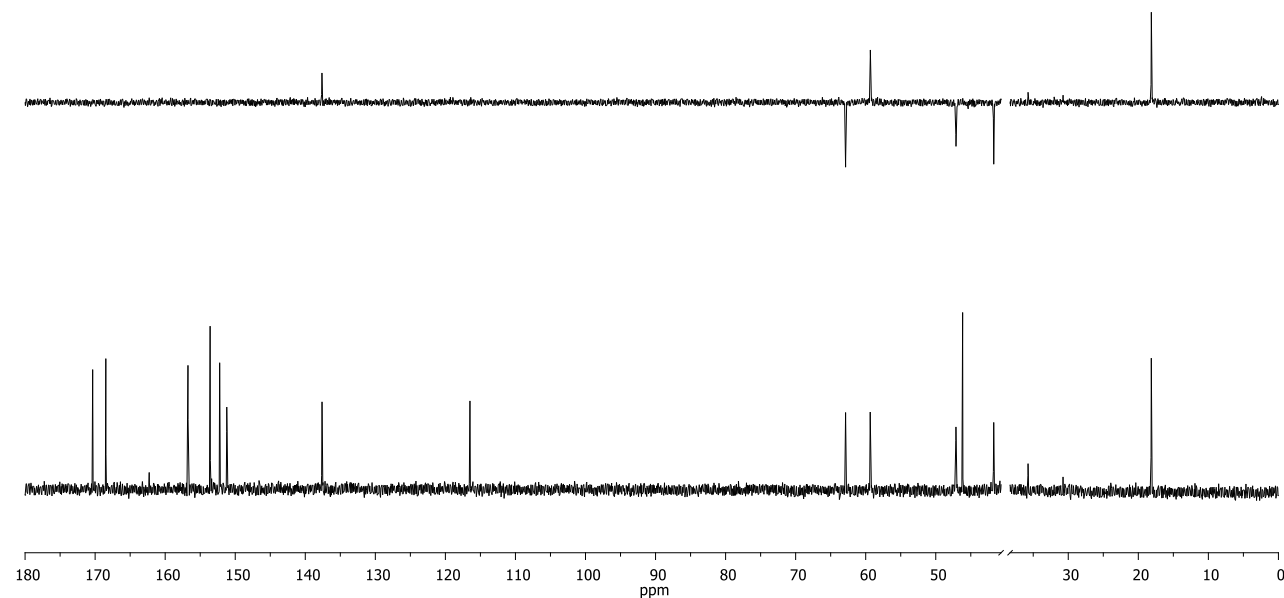


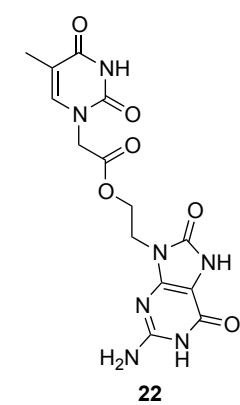

22

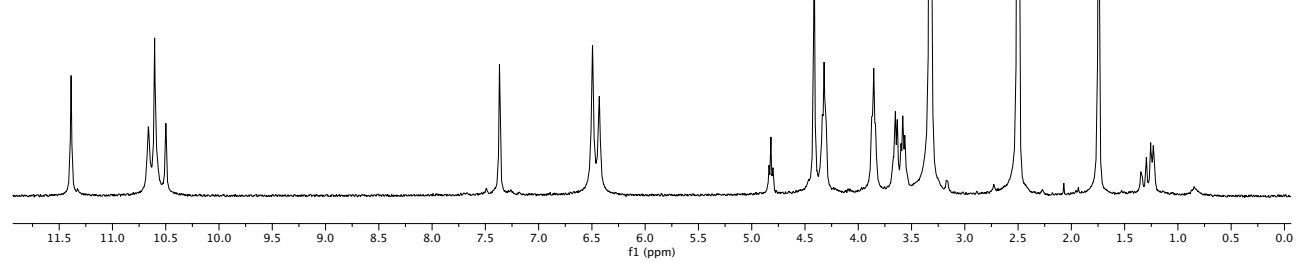




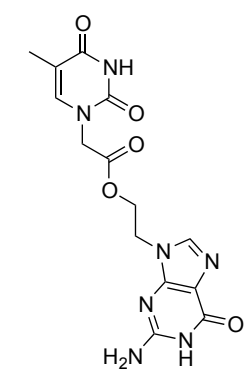

23
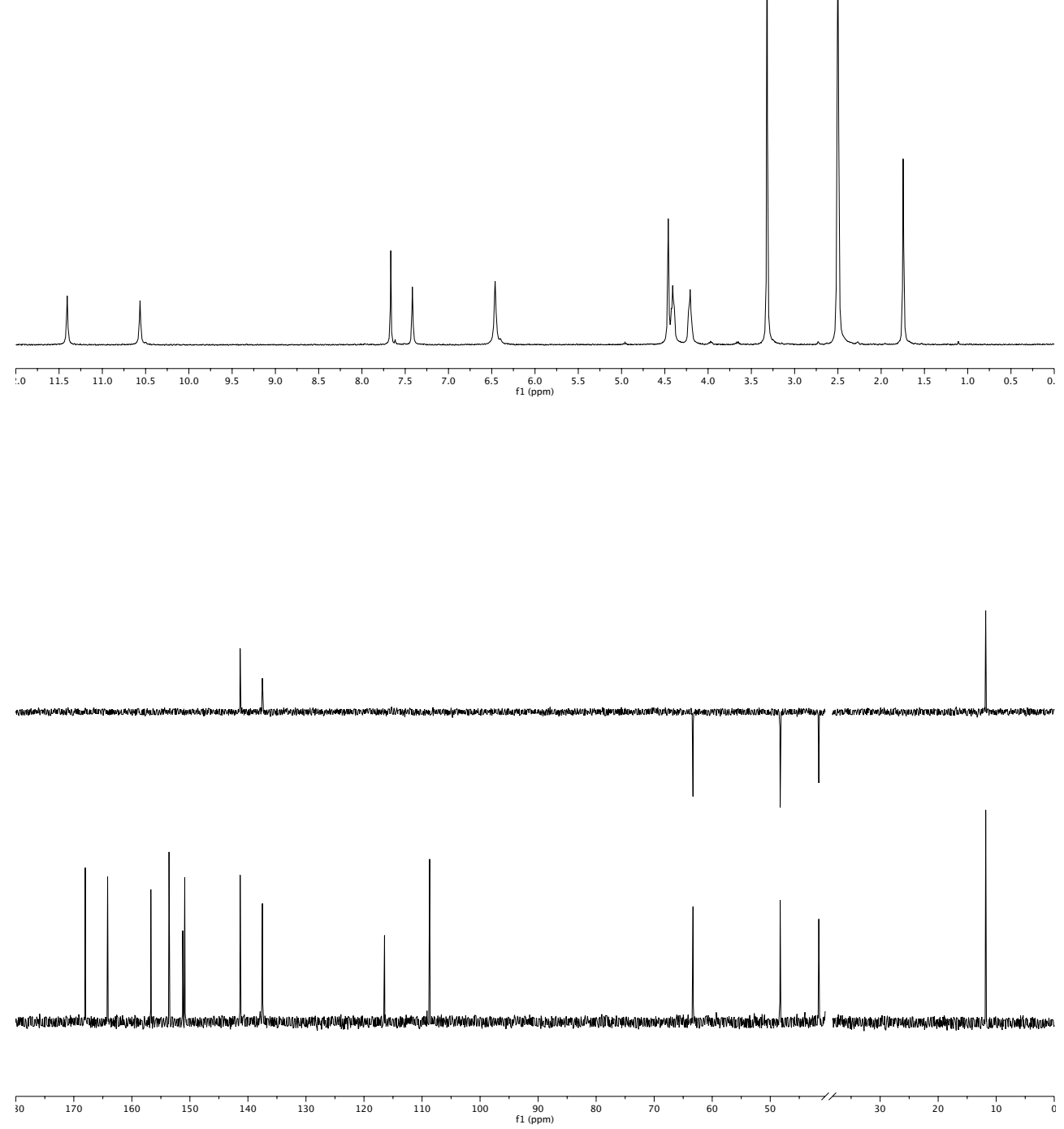


\subsection{References}

1. Gilbert, W., The RNA world. Nature 1986, 319, 618.

2. Chen, X.; Li, N.; Ellington, A. D., Ribozyme catalysis of metabolism in the RNA world. Chem. Biodiversity 2007, 4, 633-655.

3. Chinnapen, D. F.; Sen, D., A deoxyribozyme that harnesses light to repair thymine dimers in DNA. Proc. Natl. Acad. Sci. 2004, 101, 65-69.

4. Holman, M. R.; Ito, T.; Rokita, S. E., Self-repair of thymine dimer in duplex DNA. J. Am. Chem. Soc. 2007, 129, 6-7.

5. Steenken, S.; Jovanovic, S. V., How easily oxidizable is DNA? One-electron reduction potentials of adenosine and guanosine radicals in aqueous solution. J. Am. Chem. Soc. 1997, 119, 617-618.

6. Cannistraro, V. J.; Taylor, J. S., Acceleration of 5-methylcytosine deamination in cyclobutane dimers by $\mathrm{G}$ and its implications for UV-induced C-to-T mutation hotspots. J. Mol. Biol. 2009, 392, 1145-1157.

7. Pan, Z.; Hariharan, M.; Arkin, J. D.; Jalilov, A. S.; McCullagh, M.; Schatz, G. C.; Lewis, F. D., Electron donor-acceptor interactions with flanking purines influence the efficiency of thymine photodimerization. J. Am. Chem. Soc. 2011, 133, 2079320798.

8. Pan, Z.; Chen, J.; Schreier, W. J.; Kohler, B.; Lewis, F. D., Thymine dimer photoreversal in purine-containing trinucleotides. J. Phys. Chem. B 2012, 116, 698-704.

9. Middleton, C. T.; De la Harpe, K.; Su, C.; Law, Y. K.; Crespo-Hernández, C. E.; Kohler, B., DNA excited-state dynamics: from singlet base to the double helix. Annu. Rev. Phys. Chem. 2009, 60, 217-239.

10. Bucher, B. D.; Kufner, C. L.; Schlueter, A.; Carell, T., UV-induced charge transfer states in DNA promote sequence selective self-repair. J. Am. Chem. Soc. 2016, 138, 186-190.

11. Nguyen, K. V.; Burrows, C. J., A prebiotic role for 8-oxoguanosine as a flavin mimic in pyrimidine dimer photorepair. J. Am. Chem. Soc. 2011, 133, 14586-14589. 
12. Nguyen, K. V.; Burrows, C. J., Whence flavins? Redox-active ribonucleotides link metabolism and genome repair to the RNA world. Acc. Chem. Res. 2012, 45, 2151 2159.

13. Saito, I.; Nakamura, T.; Nakatani, K.; Yoshioka, Y.; Yamaguchi, K.; Sugiyama, H., Mapping of the hot spots for DNA damage by one-electron oxidation: efficacy of GG doublets and GGG triplets as a trap in long-range hole migration. J. Am. Chem. Soc. 1998, 120, 12686-12687.

14. Zhang, Y.; Dood, J.; Beckstead, A.; Li, X. B.; Nguyen, K. V.; Burrows, C. J.; Improta, R.; Kohler, B., Photoinduced electron transfer in DNA: Charge shift dynamics between 8-oxo-guanine anion and adenine. J. Phys. Chem. B 2015, 119, 74917502.

15. Lu, Z.; Beckstead, A.; Kohler, B.; Matsika, S., Excited state relaxation of neutral and basic 8-oxoguanine. J. Phys. Chem. B 2015, 119, 8293-8301.

16. Tuna, D.; Domcke, W., Excited-state deactivation in 8-oxo-deoxyguanosine: comparison between anionic and neutral forms. Phys. Chem. Chem. Phys. 2016, 18, 947956.

17. Wu, X.; Karsili, T. N. V.; Domcke, W., Role of electron-driven protontransfer processes in the ultrafast deactivation of photoexcited anionic 8-oxoguanineadenine and 8-oxoguanine-cytosine base pairs. Molecules 2017, 22, 135-149.

18. Zhang, Y.; Dood, J.; Beckstead, A.; Chen, J.; Li, X. B.; Burrows, C. J.; Lu, Z.; Matsika, S.; Kohler, B., Ultrafast excited-state dynamics and vibrational cooling of 8oxo-7,8-dihydro-2'-deoxyguanosine in $\mathrm{D}_{2} \mathrm{O}$. J. Phys. Chem. A 2013, 117, 12851-12857.

19. Zhang, Y.; Dood, J.; Beckstead, A.; Li, X. B.; Nguyen, K. V.; Burrows, C. J.; Improta, R.; Kohler, B., Efficient UV-induced charge separation and recombination in an 8-oxoguanine-containing dinucleotide. Proc. Natl. Acad. Sci. 2014, 111, 11612 11617.

20. Changenet-Barret, P.; Gustavsson, T.; Improta, R.; Markovitsi, D., Ultrafast excited-state deactivation of 8-hydroxy-2'-deoxyguanosine studied by femtosecond 
fluorescence spectroscopy and quantum-chemical calculations. J. Phys. Chem. A 2015, 119, 6131-6139.

21. Liu, Z.; Tan, C.; Guo, X.; Kao, Y. T.; Li, J.; Wang, L.; Sancar, A.; Zhong, D., Dynamics and mechanism of cyclobutane pyrimidine dimer repair by DNA photolyase. Proc. Natl. Acad. Sci. 2011, 108, 14831-14836.

22. Kim, S. T.; Hartman, R. F.; Rose, S. D., Solvent dependence of pyrimidine dimer splitting in a covalently linked dimer-indole system. Photochem. Photobiol. 1990, 52, 789-794.

23. Kim, S. T.; Rose, S. D., Pyrimidine dimer splitting in covalently linked dimerarylamine systems. Photochem. Photobiol. 1992, 12, 179-191.

24. Hartzfeld, D. G.; Rose, S. D., Efficient pyrimidine dimer radical anion splitting in low polarity solvents. J. Am. Chem. Soc. 1993, 115, 850-855.

25. Epple, R.; Wallenborn, E.; Carell, T., Investigation of flavin-containing DNArepair model compounds. J. Am. Chem. Soc. 1997, 119, 7440-7451.

26. Butenandt, J.; Epple, R.; Wallenborn, E.; Eker, A. P.; Gramlich, V.; Carell, T., A comparative repair study of thymine- and uracil-photodimers with model compounds and a photolyase repair enzyme. Chem. Eur. J. 2000, 6, 62-72.

27. Cichon, M. K.; Arnorld, S.; Carell, T., A (6-4) photolyase model: repair of DNA (6-4) lesions requires a reduced and deprotonated flavin. Angew. Chem. Int. Ed. 2002, 41, 767-770.

28. Song, Q. H.; Tang, W. J.; Hei, X. M.; Wang, H. B.; Guo, Q. W.; Yu, S. Q., Efficient photosensitized splitting of thymine dimer by a covalently linked tryptophan in solvents of high polarity. Eur. J. Org. Chem. 2005, 1097-1106.

29. Song, Q. H.; Wang, H. B.; Tang, W. J.; Guo, Q. W.; Yu, S. Q., Model studies of the (6-4) photoproduct photoreactivation: efficient photosensitized splitting of thymine oxetane units by covalently linked tryptophan in high polarity solvents. Org. Biomol. Chem. 2006, 4, 291-298. 
30. Belmadoui, N.; Encinas, S.; Climent, M. J.; Gil, S.; Miranda, M. A., Intramolecular interactions in the triplet excited states of benzophenone-thymine dyads. Chem. Eur. J. 2006, 12, 553-561.

31. Tang, W. J.; Guo, Q. X.; Song, Q. H., Origin of solvent dependence of photosensitized splitting of a cyclobutane pyrimidine dimer by a covalently linked chromophore. J. Phys. Chem. B 2009, 113, 7205-7210.

32. Kao, Y. T.; Song, Q. H.; Saxena, C.; Wang, L.; Zhong, D., Dynamics and mechanism of DNA repair in a biomimetic system: flavin-thymine dimer adduct. $J$. Am. Chem. Soc. 2012, 134, 1501-1503.

33. Shafirovich, V.; Cadet, J.; Gasparutto, D.; Dourandin, A.; Huang, W.; Geacintov, N. E., Direct spectroscopic observation of 8-oxo-7,8-dihydro-2'deoxyguanosine radicals in double-stranded DNA generated by one-electron oxidation at a distance by 2-aminopurine radicals. J. Phys. Chem. B 2001, 105, 586-592.

34. Joseph, A.; Prakash, G.; Falvey, D. E., Model studies of the (6-4) photoproduct photolyase enzyme: laser flash photolysis experiments confirm radical ion intermediates in the sensitized repair of thymine oxetane adducts. J. Am. Chem. Soc. 2000, 122, 11219-11225.

35. Guo, X.; Liu, Z.; Song, Q.; Wang, L.; Zhong, D., Dynamics and mechanism of UV-damaged DNA repair in indole-thymine dimer adduct: molecular origin of low repair quantum efficiency. J. Phys. Chem. B 2015, 119, 3446-3455.

36. Montalti, M.; Credi, A.; Prodi, L.; Gandolfi, T., Handbook of Photochemistry. 3rd edition. CRC press 2006, 601-604.

37. Yeh, S. R.; Falvey, D. E., Model studies of DNA photorepair: radical anion cleavage of thymine dimers probed by nanosecond laser spectroscopy. J. Am. Chem. Soc. 1991, 113, 8558-8560.

38. Sancar, A., Structure and function of DNA photolyase and cryptochrome blue-light photoreceptors. Chem. Rev. 2003, 103, 2203-2207. 
39. Boussicault, F.; Kruger, O.; Robert, M.; Wille, U., Dissociative electron transfer to and from pyrimidine cyclobutane dimers: An electrochemical study. Org. Biomol. Chem. 2004, 2, 2742-2750.

40. Boussicault, F.; Robert, M., Electrochemical approach to the repair of oxetanes mimicking DNA (6-4) photoproducts. J. Phys. Chem. B 2006, 110, $21987-$ 21993.

41. Prakash, G.; Falvey, D. E., Model studies of the (6-4) photoproduct DNA photolyase: synthesis and photosensitized splitting of a thymine-5,6-oxetane. J. Am. Chem. Soc. 1995, 117, 11375-11376. 

Chapter 6:

8-oxoGuanine as a Potential

Intrinsic Photosensitizer for the Repair of T(6-4)C Photoproduct 



\subsection{Introduction}

The previous chapters have focused on models to mimic the PET step involved in the photolyase repair mechanism. As a step further, it would be interesting to study how these models influence the structure of the DNA double stranded helix and how they can be repaired in short DNA strands by electron transfer.

In principle, an excited-state chromophore can inject a charge into DNA through oxidation or reduction of a proximal nucleobase to yield a radical cation or anion that may migrate in processes described as hole transfer $(\mathrm{HT})$ or excess electron transfer (EET), respectively. ${ }^{1}$ As mentioned in Chapter 1, the oxidative process is relevant in the formation of DNA damage2; however, EET into DNA is also an important process as it is associated with repair of UV lesions. ${ }^{3}$ There are three mechanisms proposed for photoinduced electron transfer into DNA according to the energetic levels of donor-bridge-acceptor system: (i) the molecular wire, (ii) the superexchange mechanism and (iii) the hopping model. In all cases, a photoexcited donor with a suitable energetic level is used in order to initiate the charge transfer. ${ }^{1,4}$ Figure 6.1 shows that the energetic levels of the bridge in relation to those of the donor and acceptor determine the mechanism. In case of a molecular wire, the bridge states are energetically comparable to the level of the donor, and the electron can be injected into the bridge and moves incoherently to the acceptor. By contrast, in the superexchange mechanism, the brigde states are lying above those of the donor, the electron is transferred in one coherent jump and is never localized within the bridge. As a consequence, it can be expected that the electron transfer rate is distance-dependent. Finally, if the bridge state is energetically comparable to that of the photoexcited donor, an electron transport via hopping could occur as an alternative mechanism to the molecular wire. By contrast, the electron is not delocalized within the bridge during the electron hopping, which consists in a multistep process of charge, injection, charge transport and charge trapping. ${ }^{1}$ 

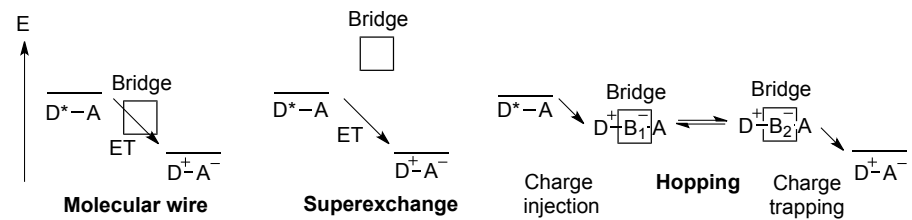

Figure 6.1. Comparison of basic mechanisms of electron transfer and transport: molecular wire, superexchange and hopping mechanism. (D: donor, A: acceptor, bridge: DNA bases, ET: electron transfer).

Excess of electron through DNA duplex has been intensively investigated.1, 5-8 A hopping mechanism was proposed for the DNA-mediated transport over long distances involving all base pairs ( $\mathrm{T}-\mathrm{A}$ and $\mathrm{C}-\mathrm{G})$ and the pyrimidine radical anions $\mathrm{C}^{\bullet-}$ and $\mathrm{T}^{\bullet-}$ as intermediate electron carriers. ${ }^{9}$ This theory was based on the relative reducibility of DNA bases, $\mathrm{T}\left(\mathrm{E}_{\text {red }}=-2.18 \mathrm{~V}\right.$ vs. NHE $)>\mathrm{C}>\mathrm{A}>\mathrm{G}\left(\mathrm{E}_{\text {red }}=-2.76 \mathrm{~V}\right.$ vs. $\mathrm{NHE})^{10}$, being pyrimidine bases $\mathrm{T}$ and $\mathrm{C}$ more easily reduced than the purine bases.

Therefore, for charge injection, compounds with suitable oxidation potential in the singlet excited state have been used as photoexcitable electron donors in DNA. In this context, flavin ${ }^{11}\left(\mathrm{E}_{\mathrm{ox}}^{\mathrm{D}^{*}}=-2.8 \mathrm{~V}\right.$ vs. NHE$)$, naphthalene diamine ${ }^{6}\left(\mathrm{E}_{\mathrm{ox}}^{\mathrm{D} *}=-2.6 \mathrm{~V}\right.$ vs. $\mathrm{NHE})$, stilbene diether ${ }^{12}\left(\mathrm{E}_{\mathrm{ox}}^{\mathrm{D}^{*}}=-2.3 \mathrm{~V}\right.$ vs. NHE$)$, phenothiazine ${ }^{13}\left(\mathrm{E}_{\mathrm{ox}}^{\mathrm{D}^{*}}=-2.0 \mathrm{~V}\right.$ vs. $\mathrm{NHE})$, pyrene ${ }^{5}\left(\mathrm{E}_{\mathrm{ox}}^{\mathrm{D}^{*}}=-1.8 \mathrm{~V}\right.$ vs. NHE) have been covalently attached to oligonucleotides, and the electron transport has been monitored using acceptors, i.e. chemical trapping probes of electron. Pyrimidine bases have similar reduction potentials as the electron donors, thus, none can serve as an electron traps. However cyclobutane thymine dimers ${ }^{14-17}$, thymine derived-oxetane ${ }^{18-19}$, halouracils ${ }^{6,20}$, thymine glycol ${ }^{21}$ or nitrobenzene $e^{5}$ have been employed in this role to investigate electron transport along the DNA helix. All these compounds suffer an irreversible chemical change informing that they have received an extra electron. The thymine dimer and oxetane undergo cycloreversion, the halouracils release halide ions and thymine glycol looses the hydroxyl groups. This way, it is possible to measure and compare electron transfer effi- 
ciencies by HPLC analysis, electrophoresis, fluorescence quenching or laser flash photolysis.

Among the donor-DNA-acceptor systems mentioned above, the flavin-DNAcyclobutane thymine dimer or oxetane systems are of particular interest as they mimic the DNA repair process by photolyase. They have been used to elucidate the distance and sequence dependence of DNA-mediated electron transfer efficiency. ${ }^{16,19}$ In these systems, the dimer (or oxetane) and flavin were separated by A-T bridges and photoirradiation of the reduced and deprotonated flavin $\left(\mathrm{FADH}^{-}\right)$causes the injection of the excess electron to the nearby thymine, after the hopping process through DNA using pyrimidines as stepping stones, the electron is trapped by the dimer (or oxetane). This causes cycloreversion, which was evidenced by HPLC analysis. Interestingly, in theses systems no by-products were detected, which supported the idea that DNA is more stable towards reduction than towards oxidation processes.

In this context, we propose to investigate the EET into DNA containing the azetidine model (AZT, Figure 6.2), described in the previous chapters, as electron trap. As mentioned in Chapter 3, spectroscopic and photochemical studies showed that injection of an electron into this heterocycle is able to induce the photocycloreversion process leading to the formation of the initial pyrimidines. ${ }^{22}$

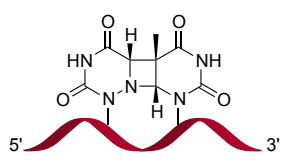

Figure 6.2. Structure of AZT inserted into an oligonucleotide.

Regarding to the electron donors studied throughout this Thesis, we selected 8oxoGuanine $(\mathrm{OG})$ as the best candidate since it (i) has a good oxidation potential in the singlet excited state $\left(\mathrm{E}_{\mathrm{ox}}^{\mathrm{D}^{*}}=-3.3 \mathrm{~V}\right.$ vs. $\left.\mathrm{NHE}\right)$, (ii) has a red-shifted absorption spectrum in a spectral window that does not produce direct photoreversal of AZT, (iii) 
Chapter 6: 8-oxoGuanine as a Potential Intrinsic Photosensitizer for the Repair of T(6-4)C photoproduct

OG phosphoramidite is commercially available what eases the oligonucleotide synthesis and (iv) this compound is not expected to perturb significantly the B-DNA duplex conformation, which is essential for the electron migration process. ${ }^{23}$ Altogether, these data show that OG-AZT (electron donor-trap) system embedded in DNA might be of interest to study the repair process.

Regarding to DNA photolyases, DNA-binding and -repair studies have been achieved with modified cyclobutane thymine dimers and (6-4) lesions in order to gain deeper understanding of the lesion recognition and repair steps. In the case of the cyclobutane dimers, the phosphodiester group linking 3' and 5' sugars was replaced with isosteric formacetal to confirm that the central phosphate is not required for the recognition by CPD photolyases. ${ }^{24}$ For 6-4PP, binding studies where the $\mathrm{C} 2$ carbonyl group of the 3'-pyrimidone was replaced with an iminium cation showed that, despite an efficient binding, (6-4) photolyase was not able to achieve the repair to the original bases. ${ }^{25}$ Interestingly, a thiethane four-membered ring, formed between thymidine and 4-thiothymidine inserted in an oligonucleotide, was also used as substrate for the (6-4) photolyase reaction; however; the binding to the enzyme was not efficient, avoiding the repair to take place. ${ }^{26}$ In spite of their potential importance during the repair of 64PPs at TC sequences, the repair of azetidine by photolyases has not been reported until now. With this background, it would be interesting to explore this enzymatic photoreaction using our azetidine AZT, which also is an aza analog of CPD, as substrate for the CPD and (6-4) photolyases.

\subsection{Results and discussion}

\subsubsection{Synthesis of AZT}

Up to now, no report exists on the incorporation of an azetidine moiety in an oligonucleotide. Thus, the first step of this study consisted in developing a method for the synthesis of our azetidine-derived oligomer (AZT). As previously described in 
Chapter 3, this ring can be obtained through a photosensitized [2+2] photocycloaddition between 6-azauracil and thymine. However, synthesis of the desired building block and its subsequent incorporation in the oligomer by means of the typical solidstate phosphoramidite method was not straightforward because of the instability of the heterocycle during the 12-steps required in the synthesis. Therefore, we settled on generating photochemically AZT in situ using tailor-made oligonucleotides, which include a 6-azauracil, as a simple and convenient method. It was performed during my second stay in the Cynthia Burrow's group at the University of Utah.

Firstly, oligonucleotides containing 6-azauracil were synthesized on a DNA synthesizer by phosphoramidite method, linking nucleotides one by one in the 3 ' $\rightarrow 5$ ' direction, and using a repeated four-step cycle of detritylation, coupling, capping and oxidation for each A, C, T or G addition. Properly-protected nucleoside 3'phosphoramidites were used as building blocks for chain elongation. The 6-azauracil nucleotide was incorporated at a specific site of oligonucleotide by means of its phosphoramidite building block, which is commercially available from Berry (Figure 6.3). By contrast with $\mathrm{U}$ or $\mathrm{T}$, this phosphoramidite includes 0 -anisoyl protecting group at $\mathrm{N} 3$ to avoid its deprotonation at neutral $\mathrm{pH} .{ }^{27}$

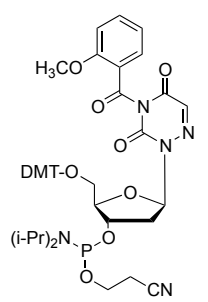

Figure 6.3. Structure of commercial 6-azauracil phosphoramidite building block.

The oligonucleotide synthesis is depicted in Scheme 6.1. At the beginning, the first protected nucleoside is pre-attached to the resin. Then, the 5'-dimethoxytrityl (DMT) protecting group is removed and the free 5'-OH attacks the phosphorus of the incoming second nucleoside, displacing its diisopropylamino group. In step 3, 
unreacted 5'-OH is acetylated, preventing elongation of sequences with deletion mutations, i.e. lacking of the second nucleobase. After that, the unstable phosphite triester is converted to a stable phosphate triester; and the next cycle is run until the desired sequence is completed. Once the last nucleotide is attached, removing of the many protecting groups that are still attached, such as DMT of 5'-OH, cyanoethyl phosphate or specific protecting groups of exocyclic primary amino groups of A, C, G and 6-azauracil is achieved, and, the oligonucleotides are cleaved from the solid support and are ready for purification by reverse-phase HPLC.

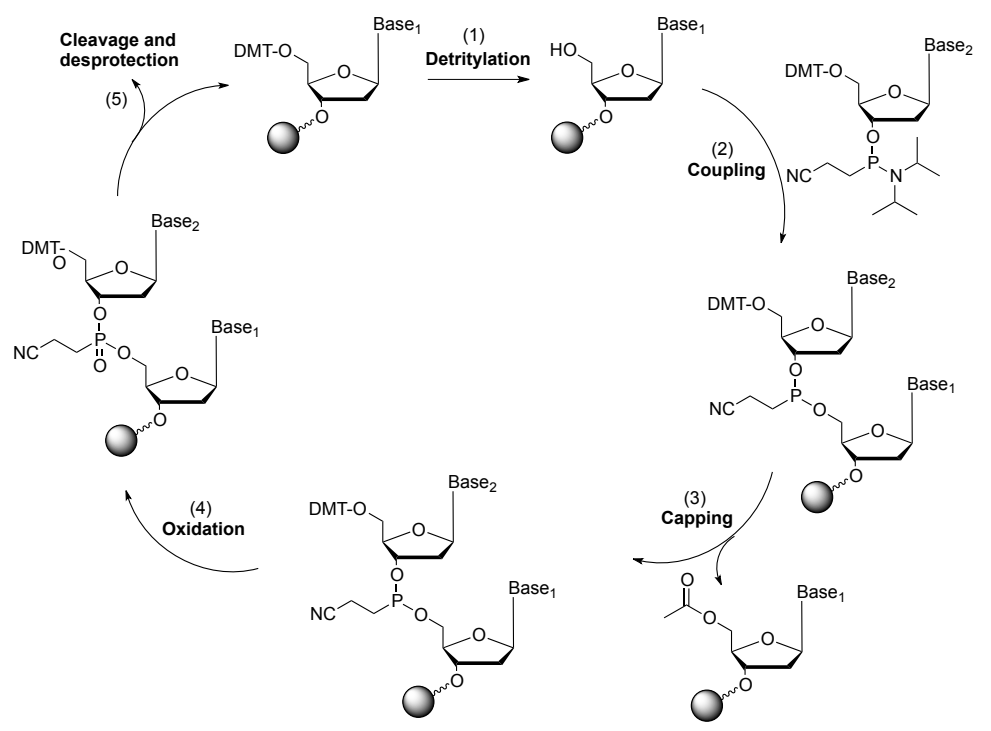

Scheme 6.1. The phosphoramidite oligonucleotide synthesis cycle: (1) trichloroacetic acid, $\mathrm{CH}_{2} \mathrm{Cl}_{2}, 50 \mathrm{~s}$; (2) tetrazole, $\mathrm{MeCN}, 30 \mathrm{~s}$; (3) $\mathrm{Ac}_{2} \mathrm{O} /$ pyridine/THF, Nmethylimidazole, MeCN, 30s; (4) $\mathrm{I}_{2}, \mathrm{H}_{2} \mathrm{O}$ /pyridine/THF, 45s; (5) $\mathrm{NH}_{4} \mathrm{OH}$ (cc), $55{ }^{\circ} \mathrm{C}$, $5 h$.

We planned to synthetize four oligonucleotides, two short (6-mer) and two long (18-mer), containing the 6-azauracil nucleotide at the 5'-side or 3'-side of a thymine nucleobase (Scheme 6.2). Moreover, the selected sequences only contain a dipyrimi- 
dine site to ensure that, the photosensitized [2+2] cycloaddition which allows formation of $\mathbf{A Z T}$, takes place exclusively at the desired $\mathbf{U}^{*}-\mathrm{T}$ site, and does not produce $\mathrm{CPD}$ at another position. Unfortunately, synthesis of 6-mer strands was not efficient enough and, thus, the purification step was too tedious to get the concentration needed for the following experiments. By contrast, 18-mers were pure enough to be used without further purification.

\section{AF1 5'-CACAGCAU*TACAGTACAC-3'}

\section{AF2 5'-CACAGCATU*ACAGTACAC-3'}

\section{AF3 5'-CAU*'TAC-3'}

\section{AF4 5'-CATU*AC-3'}

Scheme 6.2. Sequences of synthetized oligonucleotides.

Secondly, the AZT was obtained through photosensitized [2+2] cycloaddition between 6-azauracil and thymine, as described in Chapter 3, using the oligonucleotides AF1 and AF2 (Scheme 6.3 for AF1).

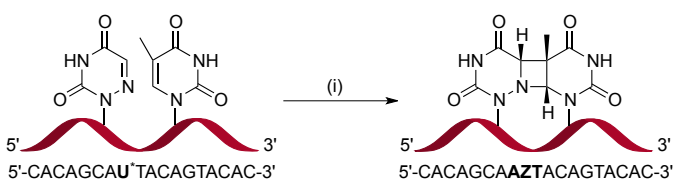

Scheme 6.3. Synthetic strategy to prepare AF1-AZT: (i) acetophenone, $\boldsymbol{\lambda}>300$, $\mathrm{N}_{2}, 0^{\circ} \mathrm{C}, 2 \mathrm{~h}$.

This approach has been successfully employed to prepare site-specific cis-syn $\mathrm{T}<>\mathrm{T}$ in oligonucleotides. ${ }^{28}$ However, acetone has been reported to produce sideproducts $^{29}$, and it has a low boiling point and can evaporate during the purging step. To circumvent these drawbacks, acetophenone was chosen as triplet photosensitizer (b.p. $202^{\circ} \mathrm{C}$ ). Thus, a deaerated solution containing AF1 (or AF2) and acetophenone 
was irradiated at $\lambda>300 \mathrm{~nm}$ in an ice bath. The progress of the reaction was followed by reverse phase HPLC monitoring the increase of new peaks, the main peak might be the cis-syn isomer and the other one, the trans-syn counterpart as reported for cyclobutane thymine dimer (Figure 6.4, left). ${ }^{28,} 30$ Once purified by reversed phase HPLC, formation of azetidine was confirmed by direct irradiation at $254 \mathrm{~nm}$ of AZT-AF1 that, as shown in Figure 6.4 right, regenerates the initial AF1 oligonucleotide.
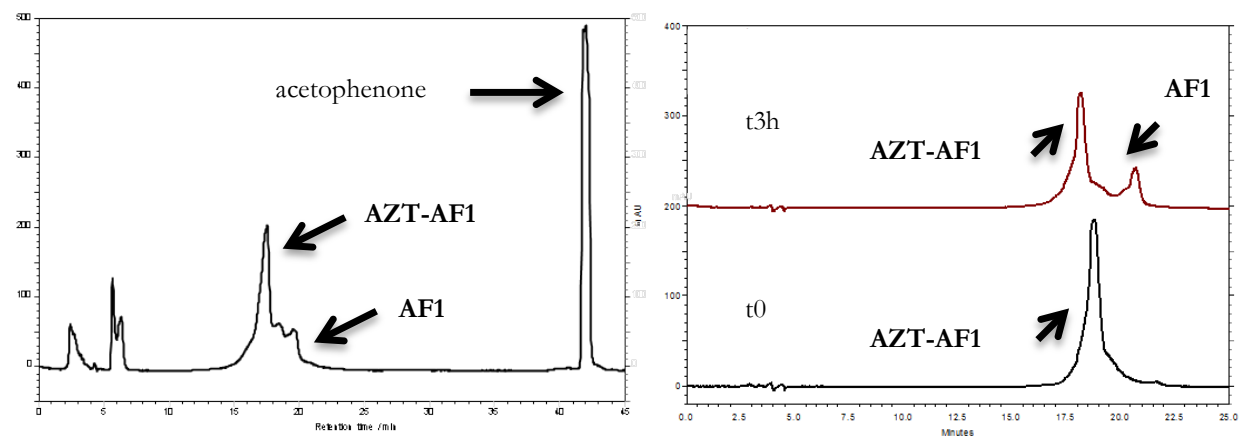

Figure 6.4. HPLC analysis of the acetophenone-photosensitized formation of AZT-AF1 at $\lambda>300 \mathrm{~nm}$ for $2 \mathrm{~h}$ (left) and its photocycloreversion irradiating at $\lambda=$ $254 \mathrm{~nm}$ for $3 \mathrm{~h}$ to regenerate AF1 (right).

\subsubsection{Photorepair of AZT by OG}

As mentioned previously, OG was selected as the intrinsic reducing agent to inject, after excitation, an electron into DNA until reaching AZT, which will act as electron trap. The easiest way to study this process is by inserting the electron donor and acceptor in the same strand. However, the presence of OG in AF1 or AF2 might interfere during the in situ generation of AZT. This way, the dependence of the repair process can be investigated on base pair stability and directional effect sequence by changing the location of the base pair of OG at 5' or 3'-side to AZT. Thus, the oxidized guanine was introduced in the complementary strand at different sites (Scheme 6.4) ie. at $5^{\prime}$ (AF5) or $3^{\prime}$-side (AF6) or opposite (AF7 and AF8) to AZT. These 
Chapter 6: 8-oxoGuanine as a Potential Intrinsic Photosensitizer for the Repair of T(6-4)C photoproduct

strands were obtained by the phosphoramidite method using the commercial available phosphoramidite building block of OG. Moreover, the two strands of the designed duplexes were of different length in order to improve their separation during the HPLC analysis: AF1/AF2 are 18-mers whereas AF5-AF8 are 22-mers.

\section{AZT-AF1(AF2) 5'-CACAGCAAZTACAGTACAC-3' 18 mer \\ AF5 3'-TCTGTGTCGOAATGTCATGTGT-5' 22 mer \\ AF6 3'-TCTGTGTCGTAAOGTCATGTGT-5’ 22 mer \\ AF7 3'-TCTGTGTCGTAOTGTCATGTGT-5’ 22 mer \\ AF8 3'-TCTGTGTCGTOATGTCATGTGT-5' 22 mer}

Scheme 6.4. Sequences of the selected duplexes AZT-AF1(AF2) / AF5-AF8

In AF5 and AF6, OG can form stable base pairs with A causing very little change in stability or structure of DNA duplex, which is essential to favor the excess electron transfer process, whereas for AF7 and AF8, OG might form an unstable base pair with $\mathrm{U}^{*}$ or T. ${ }^{23}$ Regarding to the directional effect, it might be investigated the rate of repair when the base pair of OG is located immediately 5' (AF5 and AF8) or 3' (AF6 and AF7) to the AZT. Interestingly, it was reported high rate repair of $\mathrm{T}<>\mathrm{T}$ by OG in the $5^{\prime} \rightarrow 3^{\prime}$ direction. ${ }^{31}$ This fact was explained on the basis of better base stacking when $\mathrm{OG}$ is located at the 5 ' side of $\mathrm{T}<>\mathrm{T}$ dimer, which would facilitate formation of an exciplex between the electron donor and the $\mathrm{T}<>\mathrm{T}$ acceptor. ${ }^{32}$ However, $\mathrm{T}<>\mathrm{T}$ dimer cleavage by flavin does not depend on the electron transfer direction. ${ }^{33}$

Figure 6.5. shows the UV-absorption spectra of OG and AZT (obtained from the model investigated in Chapter 3). At neutral $\mathrm{pH}$, the oxidatively generated nucleobase exhibits maxima at 250 and $295 \mathrm{~nm}$, with a tail reaching the UVA region. By 
contrast, AZT does not show any absorption maximum, as expected from the saturation of the C5-C6 double bond saturation of the initial pyrimidine bases.

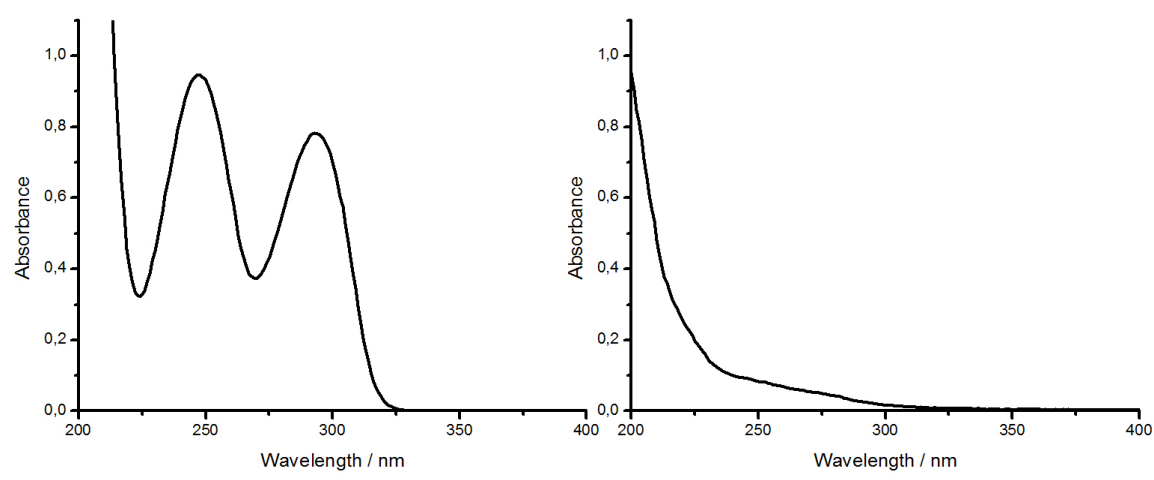

Figure 6.5. UV absorption spectra of OG in neutral phosphate buffer (left) and of AZT model (right) in acetonitrile.

Thus, the absorption spectra of AF1 and AZT-AF1 (the same for AF2) should correspond to the typical spectrum of single stranded oligonucleotide with a maximum at ca. $260 \mathrm{~nm}$. The use of polystyrene cuvettes allows cutting off the wavelengths at $\lambda<300 \mathrm{~nm}$, which is an important point in order to avoid direct excitation of AZT and its resulting photocycloreversion. In this context, a control experiment where AZT-AF1 was irradiated using these cuvettes did not show the appearance of the repaired AF1 oligonucleotide. This validates the experimental conditions that avoid photocycloreversion by direct irradiation of the heterocycle and, thus, OG is the only chromophore absorbing the incident light. In this way, once populated the singlet excited state, an electron transfer could take place from OG to the azetidine ring (Scheme 6.5), as described in the literature for the repair of cyclobutane thymine dimers. ${ }^{31,34}$ 


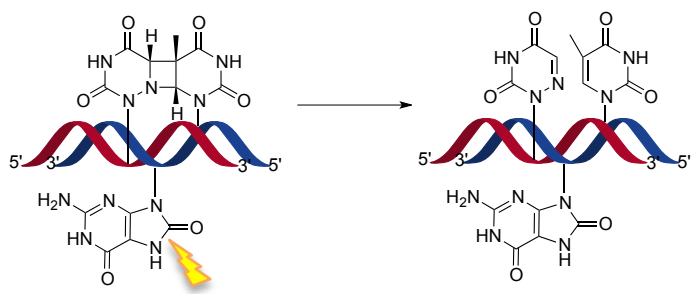

Scheme 6.5. Photorepair mechanism of AZT-AF1 from OG inserted in the complementary strand by excess electron transfer at $\lambda>300 \mathrm{~nm}$.

The first experiments were achieved with AZT-AF1, where 6-azauracil nucleotide is at the 5'-side of thymine nucleobase. The four combinations of AZT-AF1 with its complementary AF5-AF8 strand were irradiated at $\lambda>300 \mathrm{~nm}$ with UV lamps using the polystyrene cuvette and the course of the photocycloreversion process was followed by HPLC. Before irradiation, all the samples showed two peaks at 16.4 and 18.9 min that correspond to AZT-AF1 and to the complementary strand, respectively (Figure 6.6). After irradiation, a new peak, which was assigned to AF1 by comparison with the original sample, appeared at ca. $17.7 \mathrm{~min}$ and increases as a function of the irradiation time. These results show that the excitation of $O G$ in the complementary strand triggers the photocycloreversion and leads to the repair of the lesion.

Figure 6.7 represents the comparison of repaired AF1 peaks areas as a function of the OG location in the complementary strand. It should be mentioned that areas of the peaks were hardly obtained for AZT-AF1 annealed with AF7 and AF8, leading to a loss of accuracy in the results. However, it seems that the process is slightly more efficient for AF5 and AF6 where OG:A base pair is positions in 5' or 3' to the AZT site than for AF7 and AF8. Regarding to the position of the electron donor on AZTAF1 repair, the same rate of repair was observed when OG was located in 5' or 3' to the AZT lesion. Further studies will be achieved to obtain more accurate results, and to compare the results with those obtained with AZT-AF2 oligomer 
(a)

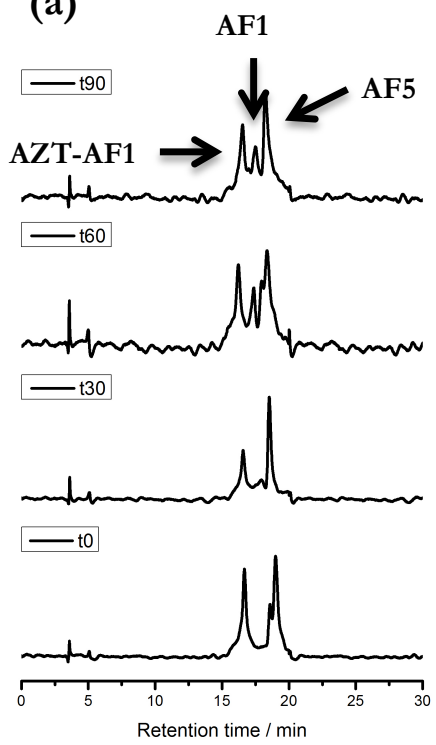

(c)
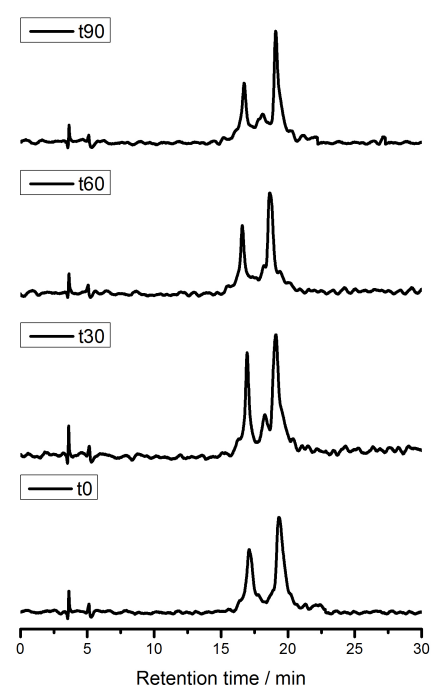

(b)

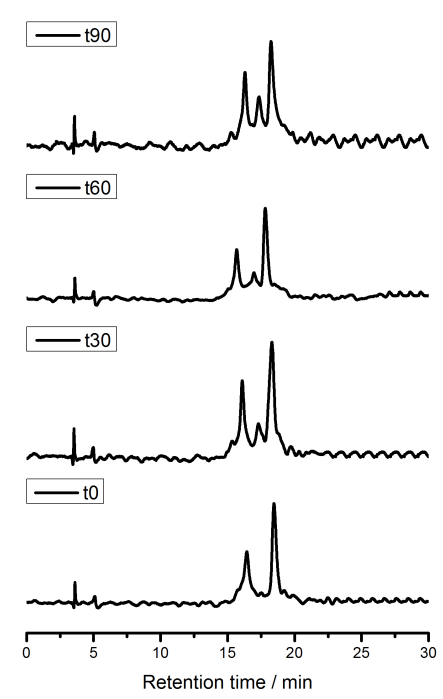

(d)

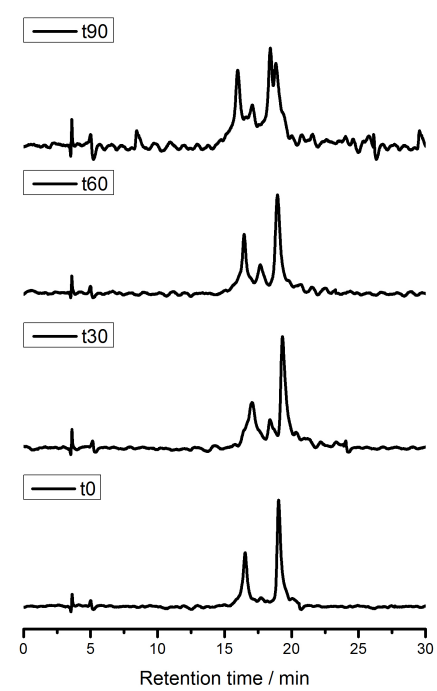

Figure 6.6. Chromatograms obtained for the irradiation of the duplexes formed between AZT-AF1 and AF5 (a), AF6 (b), AF7 (c), AF8 (d). 


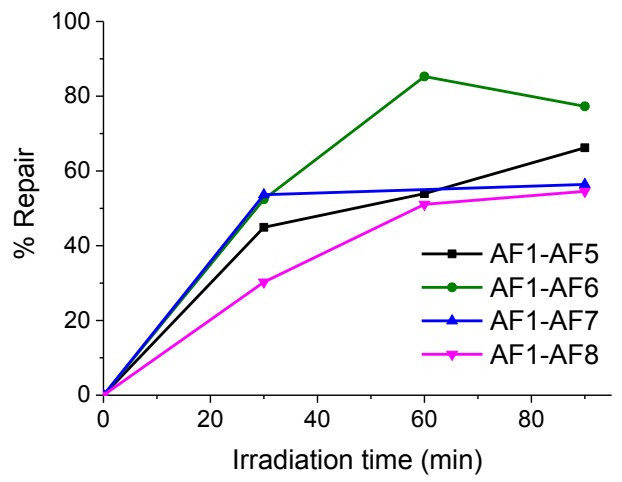

Figure 6.7. Time-dependent repair of AZT-AF1 for the four different duplexes.

\subsubsection{Photorepair of AZT by photolyases}

The AZT-AF1 oligonucleotide will also be of interest to investigate the ability of photolyases to repair this "lesion mimic". Moreover, the remarkable feature of AZT relies on its hybrid structure between a CPD and a 6-4PP azetidine intermediate. Therefore, photorepair experiments were performed in collaboration with Dr. Yunpei Yamamoto from Osaka University to evaluate the activity of Thermus thermophiles CPD photolyase (Tth) and Xenopus laevis (6-4) photolyase (X164) on AZT-AF1. In addition, two oligonucleotides containing the CPD and 6-4PP lesions (Scheme 6.6) were used as control experiments of Tth and Xl64 photolyases, respectively. These 14 mer oligonucleotides were synthetized on a DNA synthesizer using the phosphoramidite building blocks of cis-syn CPD and 6-4PP, as previously described in the literature. ${ }^{35-36}$

\section{AZT-AF1 5'-CACAGCAZTACAGTACAC-3' 18 mer CPD ATCGGCT<>TCGCGCA 14 mer 6-4PP CAGCGGT(6-4)TGCCGTG 14 mer}

Scheme 6.6. Sequences of the oligonucleotides studied for the photolyase assay. 
Chapter 6: 8-oxoGuanine as a Potential Intrinsic Photosensitizer for the Repair of T(6-4)C photoproduct

As mentioned in Chapter 1, both CPD and (6-4) photolyases contain the flavin adenine dinucleotide FAD chromophore and, thereby, the analysis of their photorepair activity of AZT-AF1 can be performed by using white light. Therefore, the 18 mer containing the AZT lesion, as well as CPD and 6-4PP 14 mers, were treated with Tth and X164 photolyases, and the reaction mixtures were analyzed by reverse phase HPLC. As shown in Figure 6.8. the CPD and 6-4PP 14 mers were converted to the undamaged 14 mer (TT 14 mer) by exposing the reaction mixture to white lighting for two hours, and the repair was confirmed by co-injection of the product with the T'T 14 mer.

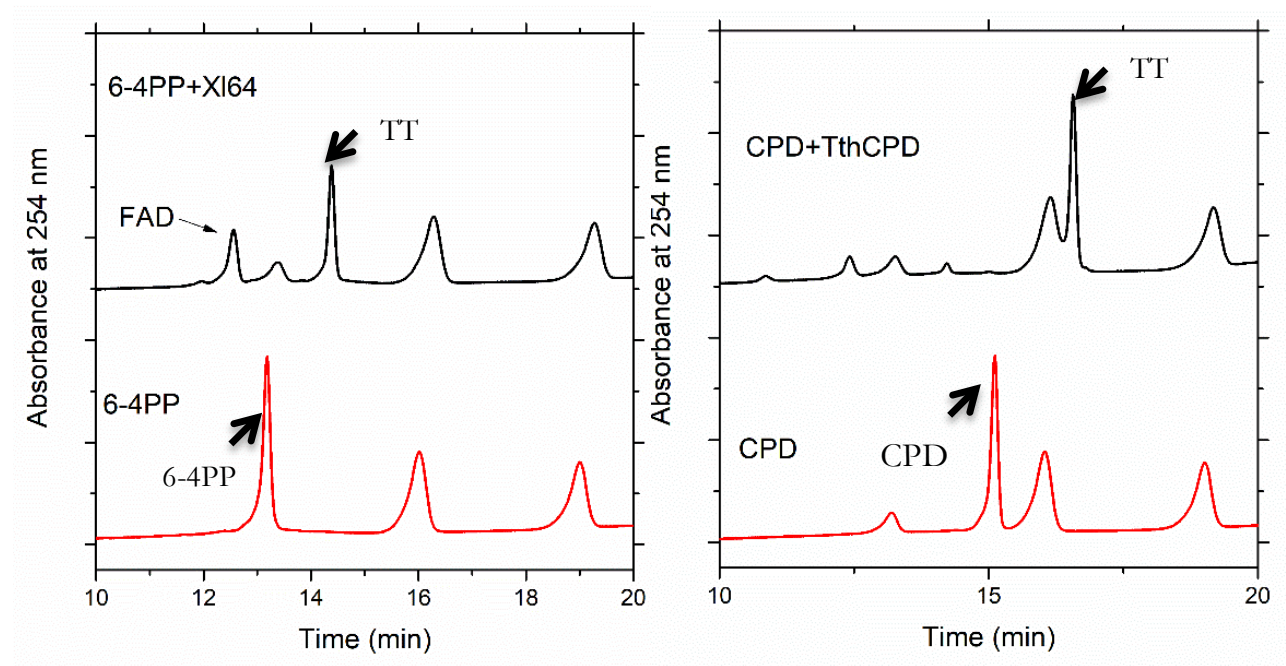

Figure 6.8. HPLC chromatograms obtained after $2 \mathrm{~h}$ of irradiation with white light of a mixture of 6-4PP oligomer with X164 (left) and CPD oligomer with Tth (right). Peaks at 13,16 and 19 min were from buffer contaminants.

Unfortunately, the peak of the AF1 was not detected for the assay with AZTAF1 in the presence of both Tth and X164 photolyases (Figure 6.9). This fact can be explained by differents reasons. The first one is that, photolyases tend to inject electrons to the 5' side of the lesions, thus, the electron accepting potential of the 5' side 
might be important. In the AZT-AF1, $\mathbf{U}^{*}$ is in the 5' side, which may significantly perturb the electron accepting potential of the AZT and thus productive electron transfer might not happen. Therefore, further experiments will be achieved with AZT-AF2 where $\mathrm{U}^{*}$ is in the 3 ' side. As a second point, it has been reported that 6azauracil incorporated in DNA has a significant influence on the base pair stability because of its low $\mathrm{pK}_{\mathrm{a}}$ value of $\mathrm{N} 3\left(\mathrm{pK}_{\mathrm{a}}=6.8\right)$ at neutral $\mathrm{pH} .{ }^{27}$ Thus, the protonation state might disturb recognition and/or repair reaction if the negatively-charged form is the major protonation state of AZT in neutral solution. Additional experiments at different $\mathrm{pH}$ values, lower than neutral, will be performed for AZT-AF1 and AZTAF2 oligomers. Moreover, binding assays will be achieved in order to know the affinity of photolyases to this azetidine lesion, which is crucial for an efficient photorepair process.

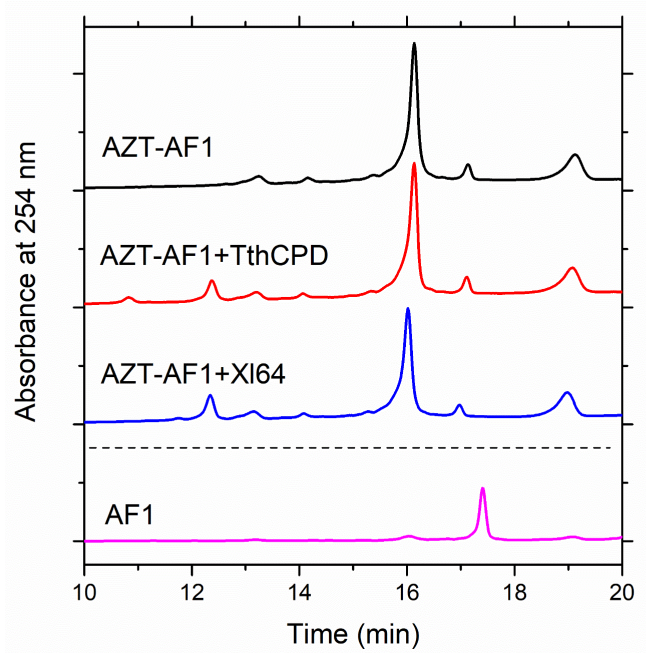

Figure 6.9. HPLC chromatograms obtained after $2 \mathrm{~h}$ of irradiation with white light of a mixture of AZT-AF1 with Tth (red) and Xl64 (blue). 
Chapter 6: 8-oxoGuanine as a Potential Intrinsic Photosensitizer for the Repair of T(6-4)C photoproduct

\subsection{Conclusions}

An azetidine incorporated into DNA was obtained for the first time. Excess electron transfer experiments have been performed using the azetidine as electron trap. Firstly, the oxidized DNA damage OG was selected as electron donor to induce the photocycloreversion of the azetidine. Initial experiments concluded that this photoreaction is possible but that it is not sequence or direction dependent. Secondly, DNA repair by photolyase were achieved using the azetidine-derived oligomer as substrate. However, further experiments are necessary to get more information in order to know if the photorepair process can take place.

\subsection{Experimental section}

\subsubsection{Oligonucleotide synthesis}

6-azauridine phosphoramidite was purchased from Berry and G, OG, T and A phosphoramidites were purchased from Glen Research. The oligonucleotides AF1AF8 were synthesized at the DNA/Peptide Core facility at the University of Utah. Oligodeoxynucleotides were cleaved and deprotected in sealed glass vials with concentrated $\mathrm{NH}_{4} \mathrm{OH}$ for $16 \mathrm{~h}$ at $55^{\circ} \mathrm{C}$ in the dark. In the cases of oligonucleotides containing OG (AF5-AF8), 0.25M $\beta$-mercaptoethanol was added to the deprotection solutions to avoid the oxidation of OG. AF5-AF8 were purified by reverse-phase HPLC on a Polaris C18 (4.6 mm i.d. x $25 \mathrm{~cm}$ length) column with linear gradient of 93:7 (50 $\mathrm{mM} \mathrm{CH}_{3} \mathrm{CO}_{2} \mathrm{NH}_{4}: \mathrm{MeCN}$ ) to $83: 17$ over $30 \mathrm{~min}$ at a $1 \mathrm{~mL} \mathrm{~min}^{-1}$ flow. AF1-AF2 were used whithout HPLC purification. The identity of oligomers was determined by TOF MS ES . Oligonucleotides CPD and 6-4PP were synthesized on an Applied Biosystems 3400 DNA synthesizer, by using the phosphoramidite building blocks of the cissyn $\mathrm{CPD}$ and 6-4PP as described previously. $35-36$ 
Chapter 6: 8-oxoGuanine as a Potential Intrinsic Photosensitizer for the Repair of T(6-4)C photoproduct

\subsubsection{Photochemical preparation of AZT oligonucleotide}

The azetidines (AZT-AF1/AZT-AF2) were prepared through acetophenone photosensitization. Thus, a $\mathrm{N}_{2}$ purged solution of $0.44 \mathrm{~mL}$ of acetophenone and 150 $\mathrm{mL}$ of an AF1 or AF2 solution ( $200 \mu \mathrm{M}$ in water) was irradiated at $313 \mathrm{~nm}$ for 2 hours in a polystyrene cuvette lying on an ice bath. The reaction was followed by reversed phase HPLC on a Zorbax Microsorb-MV C18 column $(4.6 \mathrm{~mm}$ i.d. x $25 \mathrm{~cm}$ length) with detection wavelength fixed at $260 \mathrm{~nm}$ and using a linear gradient of 93:7 (50 $\mathrm{mM} \mathrm{CH}_{3} \mathrm{CO}_{2} \mathrm{NH}_{4}: \mathrm{MeCN}$ ) to $90: 10$ over $30 \mathrm{~min}^{2}$ at a $1 \mathrm{~mL} \mathrm{~min}{ }^{-1}$ flow. The solution was lyophilized to eliminate part of the acetophenone and was purified by reverse-phase HPLC using the abovementioned conditions and then re-lyophilized.

\subsubsection{Photorepair experiments with OG}

The AZT-AF1 18 mer was resuspended in a buffer solution $(20 \mathrm{mM} \mathrm{NaPi}, 100$ $\mathrm{mM} \mathrm{NaCl}, \mathrm{pH}$ 7) to reach a concentration of $10 \mu \mathrm{M}$ and was annealed with the appropriate complementary sequences (AF5-AF8, $10 \mu \mathrm{M}$ ) by heating $2 \mathrm{~min}$ at $85^{\circ} \mathrm{C}$ and cooling in the dark to room temperature over $3 \mathrm{~h}$. The obtained double stranded oligonucleotides were irradiated with four UVB lamps (Luzchem, ranging from 280 to 400 with a maximum output at $300 \mathrm{~nm}$ ) using polystyrene cuvettes on an ice bath. The kinetics of the reaction was followed by reverse phase HPLC with the Zorbax column at $75{ }^{\circ} \mathrm{C}$ using a gradient of $50 \mathrm{mM}$ of $\mathrm{CH}_{3} \mathrm{CO}_{2} \mathrm{NH}_{4}$ : $\mathrm{MeCN}$ (93:7 to 83:17 over $30 \mathrm{~min})$. Under these conditions, the DNA duplex was denatured, the 22-mer complementary strand (AF5-AF8) being eluted at $18.9 \mathrm{~min}$, while the 18-mers AZTAF1 and AF1 have retention times of 16.4 and $17.7 \mathrm{~min}$, respectively. The peak areas, obtained by the integration of the HPLC peaks, were normalized against extinction coefficients of each strand of AZT-AF1 or AF1 and used to calculate the photorepair yield. 
Chapter 6: 8-oxoGuanine as a Potential Intrinsic Photosensitizer for the Repair of T(6-4)C photoproduct

\subsubsection{Photorepair experiments with photolyases}

Analysis of the CPD and (6-4) photolyase reaction was performed by HPLC, following the previous reported conditions. ${ }^{25} \mathrm{~A}$ solution of Thermus thermophiles CPD photolyase or Xenopus laevis (6-4) photolyase $(2 \mu \mathrm{M})$ in a buffer containing $10 \mathrm{mM}$ phosphate (pH 7.0), $100 \mathrm{mM} \mathrm{NaCl}, 5 \%$ glycerol and $10 \mathrm{mM}$ dithiothreitol, which was covered with a Pyrex lid, was illuminated on ice with $18 \mathrm{~W}$ fluorescent lamp at a distance of $15 \mathrm{~cm}$ for $30 \mathrm{~min}$. A solution of CPD, 6-4PP or AZT-AF1 oligomer $(1 \mu \mathrm{M})$ was added to the preincubated mixture, and irradiated again for $2 \mathrm{~h}$. The reaction mixture was analyzed by reverse phase HPLC with the Waters $\mu$ Bondasphere C18 column at $50{ }^{\circ} \mathrm{C}$ using a gradient of $0-40 \% \mathrm{~B}$ over $20 \mathrm{~min}(\mathrm{~A}: 5 \% \mathrm{MeCN} / 0.1 \mathrm{M}$ TEAA, B: 25\% MeCN/0.1 M TEAA), with a flow rate of $1 \mathrm{~mL} \mathrm{~min}^{-1}$.

\subsection{References}

1. Wagenknecht, H. A., Electron transfer processes in DNA: mechanisms, biological relevance and applications in DNA analytics. Nat. Prod. Rep. 2006, 23, 9731006.

2. Cadet, J.; Douki, J.; Ravanat, J.; Mascio, P., Sensitized formation of oxidatively generated damage to cellular DNA by UVA radiation. Photochem. Photobiol. 2009, 8, 903-911.

3. Sancar, A., Structure and function of DNA photolyase and cryptochrome blue-light photoreceptors. Chem. Rev. 2003, 103, 2203-2207.

4. Wagenknecht, H. A., From Mechanism to Application. Chapter 1. Wiley-VCH Ed. 2005, 1-26.

5. Maie, K.; Miyagi, K.; Takada, T.; Nakamura, M.; Yamana, K., RNA-mediated electron transfer: double exponential distance dependence. J. Am. Chem. Soc. 2009, 131, 13188-13189. 
6. Ito, T.; Rokita, S. E., Reductive electron injection into duplex DNA by aromatic amines. J. Am. Chem. Soc. 2004, 126, 15552-15559.

7. $\quad$ Elias, B.; Genereux, J. C.; Barton, J. K., Ping-pong electron transfer through DNA. Angew. Chem. Int. Ed. 2008, 47, 9067-9070.

8. Lin, S. H.; Fujitsuka, M.; Majima, T., Sequence-dependent photocurrent generation through long-distance excess-electron transfer in DNA. Angew. Chem. Int. Ed. 2016, 55, 1-4.

9. Giese, B., Long-distance electron transfer through DNA. Annu. Rev. Biochem. 2002, 71, 51-70.

10. Seidel, C. A. M., Nucleobase-specific quenching of fluorescent dyes. Nucleobase one-electron redox potentials and their correlation with static and dynamic quenching efficiencies. J. Phys. Chem. 1996, 100, 5541-5553.

11. Behrens, C.; Cichon, M. K.; Grolle, F.; Hennecke, U.; Carell, T., Excess electron transfer in defined donor-nucleobase and donor-DNA-acceptor systems. Top. Curr. Chem. 2004, 236, 187-204.

12. Lewis, F. D.; Wasielewski, M. R., Dynamics and equilibrium for single step hole transport processes in duplex DNA. Top. Curr. Chem. 2004, 236, 45-65.

13. Wagenknecht, H. A.; Fiebig, T., From Mechanism to Application. Chapter 9. WileyVCH Ed. 2005, 197-204.

14. Schwögler, A.; Burgdorf, L. T.; Carell, T., Self-repairing DNA based on a reductive electron transfer through the base stack. Angew. Chem. Int. Ed. 2000, 39, 3918-3920.

15. Breeger, S.; Hennecke, U.; Carell, T., Excess electron-transfer-based repair of a cis-syn thymine dimer in DNA is not sequence dependent. J. Am. Chem. Soc. 2004, 126, 1302-1303.

16. Behrens, C.; Burgdorf, L. T.; Schwögler, A.; Carell, T., Weak distance dependence of excess electron transfer in DNA. Angew, Chem. Int. Ed. 2002, 114, 1841-1845. 
17. Giese, B.; Carl, B.; Carl, T.; Schiemann, O.; Feresin, E., Excess electron transport through DNA: a single electron repairs more than one UV-induced lesion. Angew. Chem. Int. Ed. 2004, 43, 1848-1851.

18. Stafforst, T.; Diederichsen, U., Thymine oxetanes as charge traps for chemical monitoring of nucleic acid mediated transfer of excess electrons. Angew. Chem. Int. Ed. 2006, 45, 5376-5380.

19. Breeger, S.; von Meltzer, M.; Hennecke, U.; Carell, T., Investigation of the pathways of excess electron transfer in DNA with flavin-donor and oxetane-acceptor modified DNA hairpins. Chem. Eur. J. 2006, 12, 6469-6477.

20. Ito, T.; Rokita, S. E., Excess electron transfer from an internally conjugated aromatic amine to 5-bromo-2'-deoxyuridine in DNA. J. Am. Chem. Soc. 2003, 125, 11480-11481.

21. Ito, T.; Kondo, A.; Terada, S.; Nishimoto, S., Photoinduced reductive repair of thymine glycol: implications for excess electron transfer through DNA containing modified bases. J. Am. Chem. Soc. 2006, 128, 10934-10942.

22. Fraga-Timiraos, A. B.; Lhiaubet-Vallet, V.; Miranda, M. A., Repair of a dimeric azetidine related to the thymine-cytosine (6-4) photoproduct by electron transfer photoreduction. Angew. Chem. Int. Ed. 2016, 55, 6037-6040.

23. McAuley-Hecht, K. E.; Leonard, G. A.; Gibson, N. J.; Thomson, J. B.; Watson, W. P.; Hunter, W. N.; Brown, T., Crystal structure of a DNA duplex containing 8-hydroxydeoxyguanine-adenine base pairs. Biochemistry 1994, 33, 1026610270 .

24. Butenandt, J.; Eker, A. P. M.; Carell, T., Synthesis, crystal structure, and enzymatic evaluation of a DNA-photolesion isostere. Chem. Eur. J. 1998, 4, 642-654.

25. Yamamoto, J.; Hitomi, K.; Hayashi, R.; Getzoff, E. D.; Iwai, S., Role of the carbonyl group of the (6-4) photoproduct in the (6-4) photolyase reaction. Biochemistry 2009, 48, 9306-9312. 
26. Zhao, X.; Liu, J.; Hsu, D. S.; Zhao, S.; Taylor, J. S.; Sancar, A., Reaction mechanism of (6-4) photolyase. J. Biol. Chem. 1997, 272, 32580-32590.

27. Seela, F.; Chittepu, P., Oligonucleotides containing 6-aza-2'-deoxyuridine: synthesis, nucleobase protection, pH-dependent duplex stability, and metal-DNA formation. J. Org. Chem. 2007, 72, 4358-4366.

28. Jiang, N.; Taylor, J. S., In vivo evidence that $\mathrm{UV}$-induced $\mathrm{C}=>\mathrm{T}$ mutations at dipyrimidine sites could result from the replicative bypass of cis-syn cyclobutane dimers or their deamination products. Biochemistry 1993, 32, 472-481.

29. Mu, W.; Han, Q.; Luo, Z.; Wang, Y., Production of cis-syn thymine-thymine cyclobutane dimer oligonucleotide in the presence of acetone photosensitizer. Anal. Biochem. 2006, 353, 117-123.

30. Banerjee, S. K.; Borden, A.; Christensen, R. B.; LeClerc, J. E.; Lawrence, C. W., SOS-dependent replication past a single trans-syn T-T cyclobutane dimer gives a different mutation spectrum and increased error rate compared with replication past this lesion in uninduced cells. J. Bacteriol. 1990, 172, 2105-2112.

31. Nguyen, K. V.; Burrows, C. J., A prebiotic role for 8-oxoguanosine as a flavin mimic in pyrimidine dimer photorepair. J. Am. Chem. Soc. 2011, 133, 14586-14589.

32. Crespo-Hernández, C. E.; Cohen, B.; Kohler, B., Base stacking controls excited-state dynamics in A T DNA. Nature 2005, 436, 1141-1144.

33. Haas, C.; Kräling, K.; Cichon, M.; Rahe, N.; Carell, T., Excess electron transfer driven DNA does not depend on the transfer direction. Angew. Chem. Int. Ed. 2004, 116, 1878-1880.

34. Nguyen, K. V.; Burrows, C. J., Whence flavins? Redox-active ribonucleotides link metabolism and genome repair to the RNA world. Acc. Chem. Res. 2012, 45, 2151 2159.

35. Reyna, M. M.; Yamamoto, J.; Huang, W. C.; Tsai, M. D.; Essen, L. O.; Bessho, Y., Twist and turn: a revised structural view on the unpaired bubble of class II CPD photolyase in complex with damaged DNA. IUCrJ 2018, 5, 608-618. 
Chapter 6: 8-oxoGuanine as a Potential Intrinsic Photosensitizer for the Repair of T(6-4)C photoproduct

36. Franz, S.; Ignatz, E.; Wenzel, S.; Zielosko, H.; Putu, E. P.; Reyna, M. M.; Tsai, M. D.; Yamamoto, J.; Mittag, M.; Essen, L. O., Structure of the bifunctional cryptochrome aCRY from Chlamydomonas reinhardtii. Nucleic Acids Res. 2018, 46, 80108022. 
Chapter 7:

Instrumentation 



\subsection{Nuclear magnetic resonance (NMR)}

The ${ }^{1} \mathrm{H}$ and ${ }^{13} \mathrm{C}$ NMR spectra were measured by a $300 \mathrm{MHz}$ instrument, and $\mathrm{CDCl}_{3}$ and DMSO- $d_{6}$ were used as solvent for the spectra. The solvent signal was taken as the reference using a chemical shift of $\delta$ of ca. $7.26 \mathrm{ppm}$ and $2.50 \mathrm{ppm}$ for ${ }^{1} \mathrm{H}$ NMR and $77.16 \mathrm{ppm}$ and $39.52 \mathrm{ppm}$ for ${ }^{13} \mathrm{C}$ NMR, respectively.

\subsection{Absorption measurements}

All UV-Vis absorption spectra were recorded on a Varian Cary 50 spectrophotometer with a quartz cell of $1 \mathrm{~cm}$ optical path length.

\subsection{Fluorescence Measurements}

Steady-state fluorescence. Steady-state fluorescence experiments were carried out on a Photon Technology International (PTI) LPS-220B spectrofluorometer equipped with a Xenon lamp of $75 \mathrm{~W}$ and a monochromator that covers a range from 200 to $700 \mathrm{~nm}$. All experiments were performed under air in a quartz cuvette of $1 \mathrm{~cm}$ of optical path. The absorbance of the sensitizer was kept under 0.15 at the excitation wavelength. Stock solutions of the quenchers $(0.15 \mathrm{M})$ were prepared, so it was only necessary to add microliter volumes to the sample cell to obtain appropriate concentrations of the quencher. The bimolecular rate constants $\mathrm{k}_{\mathrm{q}}(\mathrm{SS})$ for the reaction were obtained from the Stern-Volmer plots following the equation:

$$
\mathrm{I}_{0} / \mathrm{I}=1+\mathrm{k}_{\mathrm{q}}(\mathrm{SS}) \tau_{0} \quad[\mathrm{Q}]=1+\mathrm{K}_{\mathrm{sv}}[\mathrm{Q}]
$$

where $\mathrm{I}_{0}$ and $\mathrm{I}$ are the emission intensity in the absence of $\mathrm{Q}$ and after the addition of a quencher concentration $[\mathrm{Q}]$, respectively; $\mathrm{K}_{\mathrm{SV}}\left(=\mathrm{k}_{\mathrm{q}}(\mathrm{SS}) \boldsymbol{\tau}_{0}\right)$ is the SternVolmer rate constant obtained from the slope and $\boldsymbol{\tau}_{0}$ is the lifetime of the photosensitizer in the absence of $\mathrm{Q}$. 
Time-resolved fluorescence. Measurements were performed with an EasyLife V spectrometer from OBB, equipped with a pulsed LED as excitation source; residual excitation signal was filtered in emission by using a cut-off filter. The kinetic traces were fitted by one monoexponential decay function, using a deconvolution procedure to separate them from the lamp pulse profile.

The absorbance of the sensitizer was kept at 0.15 at the excitation wavelength. Quenchers stock solutions of $0.15 \mathrm{M}$ were also used for this experiment, and the rate constants $\mathrm{k}_{\mathrm{q}}(\mathrm{TR})$ for the reaction were obtained from the Stern-Volmer plots following the equation:

$$
1 / \tau=1 / \tau_{0}+\mathrm{k}_{\mathrm{q}}(\mathrm{TR})[\mathrm{Q}]
$$

where $\tau_{0}$ is the lifetime of the photosensitizer in the absence of $\mathrm{Q}$ and $\boldsymbol{\tau}$ is the lifetime after addition of a quencher concentration [Q].

\subsection{Cyclic voltammetry}

Cyclic voltammetry measurements were performed with a VersaSTAT 3 potentiostat (Princeton Applied Research, Algete-Madrid, Spain) and using a three electrode standard configuration with glassy carbon as working electrode, platinum wire as counter electrode, and $\mathrm{Ag} / \mathrm{AgCl}$ in $3 \mathrm{M} \mathrm{NaCl}$ as reference electrode. Measurements were carried out on $\mathrm{N}_{2}$-purged acetonitrile or DMF solutions with $0.1 \mathrm{M} \mathrm{Bu}_{4} \mathrm{NClO}_{4}$ as electrolyte at a scan rate of $0.1 \mathrm{~V} \mathrm{~s}^{-1}$. Ferrocene was taken as standard and measured potentials have been referenced to the $\mathrm{E}_{1 / 2}$ potential of the ferrocinium/ferrocene $\left(\mathrm{Fc}^{+} / \mathrm{Fc}\right)$ couple of $0.425 \mathrm{~V}$ vs. $\mathrm{Ag} / \mathrm{AgCl}$ in $\mathrm{MeCN}$.

\subsection{Steady-state photolysis}

Irradiations of the samples were run using different systems: 
- A Xenon lamp (150 W or $75 \mathrm{~W})$ equipped with a monochromator from Photon Technology International (PTI) for monochromatic irradiations.

- A medium pressure mercury lamp (125 W).

- A Luzchem photoreactor (model LZC-4V) with four lamps ranging from 280 $\mathrm{nm}$ to $400 \mathrm{~nm}$ with a maximum output at $300 \mathrm{~nm}$.

- A FS40 UVB lamp with maximum output at $313 \mathrm{~nm}$ (Homophototherapy, $\mathrm{OH}, \mathrm{USA})$.

Irradiations were performed under anaerobic and aerobic conditions in a quartz cell of $1 \mathrm{~cm}$ optical path length, pyrex vessels or polystyrene cuvettes.

\subsection{HPLC analyses}

The irradiated solutions were analyzed by reverse phase HPLC using a Varian ProStar instrument equipped with a diode array detector covering a detection range from 200 to $400 \mathrm{~nm}$. Two columns were used: (1) a Mediterranean Sea C18 (4.6 mm i.d. $x 25 \mathrm{~cm}$ length, $5 \mu$ Teknokroma) with detection wavelength fixed at $270 \mathrm{~nm}$. In Chapter 3 and 4, isocratic mixture of $\mathrm{MeCN}_{\mathrm{H}} \mathrm{H}_{2} \mathrm{O}(20: 80 \mathrm{v} / \mathrm{v})$ at a flow of $1 \mathrm{~mL} \mathrm{~min}^{-1}$ was used as mobile phase. In Chapter 5, isocratic mixture of $\mathrm{MeOH}: \mathrm{H}_{2} \mathrm{O}(70: 30, \mathrm{v} / \mathrm{v})$ at a flow of $1 \mathrm{~mL} \mathrm{~min}-1$ was used for compound 10 and a linear gradient of 2:98 ( $\mathrm{MeCN}: \mathrm{H}_{2} \mathrm{O}$ ) to $65: 35$ over $30 \mathrm{~min}$ at a $1 \mathrm{~mL} \mathrm{~min}^{-1}$ flow was used for compounds $\mathbf{1 5}$, 16, 20 and 21. (2) A Zorbax Microsorb-MV C18 column (4.6 mm i.d. x $25 \mathrm{~cm}$ length) with detection wavelength fixed at $260 \mathrm{~nm}$ and using a linear gradient of 93:7 (50 mM $\mathrm{CH}_{3} \mathrm{CO}_{2} \mathrm{NH}_{4}: \mathrm{MeCN}$ ) to $83: 10$ over 30 min at a $1 \mathrm{~mL} \mathrm{~min}^{-1}$ flow was used in Chapter 6.

\subsection{UPLC-HRMS analyses}

Exact mass values were determined by using a QTof spectrometer coupled with a liquid chromatography system with a conditioned autosampler at $10{ }^{\circ} \mathrm{C}$. The separa- 
tion was carried out on an UPLC with a BEH C18 column $(50 \mathrm{~mm} \AA \sim 2.1 \mathrm{~mm}$ i.d., $1.7 \mu \mathrm{m})$ or HSS T3 column $(150 \mathrm{~mm} \AA \sim 2.1 \mathrm{~mm}, 1.8 \mu \mathrm{m})$. The ESI source was operated in positive or negative ionization mode with the capillary voltage at $1.9 \mathrm{kV}$ or 2.4 $\mathrm{kV}$, respectively. The temperature of the source and desolvation was set at 80 and 400 ${ }^{\circ} \mathrm{C}$, respectively. The cone and desolvation gas flows were 20 and $800 \mathrm{~L} \mathrm{~h}^{-1}$, respectively. All data were collected in centroid mode. Leucine-enkephalin was used as the lock mass generating an $[\mathrm{M}+\mathrm{H}]^{+}$ion $(m / z 556.2771)$ or $[\mathrm{M}-\mathrm{H}]-$ ion $(m / z 554.2615)$ at a concentration of $250 \mathrm{pg} / \mathrm{mL}$ and flow rate of $50 \mu \mathrm{L} \mathrm{min}{ }^{-1}$ to ensure accuracy during the MS analysis.

\subsection{Femtosecond broadband transient absorption spectroscopy (fs-TAS)}

A Femtosecond broadband pump-probe spectroscopy utilizing an $800 \mathrm{~nm}, 1$ $\mathrm{kHz}$ laser source (4.0 W, 100 fs pulse, Libra-HE from Coherent Inc.) was used. The fundamental beam at $800 \mathrm{~nm}$ was split into the pump and probe beams using a 98/2 beam splitter, respectively. The pump beam was tuned to $308 \mathrm{~nm}$ using an optical parametric amplifier (TOPAS, Light Conversion). The probe beam was attenuated using an optical filter wheel and focused into a continuously translating $2 \mathrm{~mm} \mathrm{CaF}_{2}$ crystal used to generate a white light continuum in the spectral window from ca. 320 to $700 \mathrm{~nm}$. The maximum overlap of the crosscorrelated pump and probe beams was determined from the stimulated Raman emission bands of acetonitrile and defined as time zero in the transient absorption spectra. The instrument response function was estimated to be $200 \pm 50$ fs from the two-photon absorption of pure methanol. A 2 $\mathrm{mm}$ fused silica cuvette with a magnetic stir bar was used to ensure homogeneity of the solutions. Samples were monitored for potential photodegradation using steadystate absorption spectroscopy (Cary 100 Bio Instrument, Agilent Technologies). A fresh solution was used if the absorbance of the solutions at the absorption maximum of each compound decreased by $5 \%$. The fs-TAS kinetic data were modeled through global fit analyses using Igor Pro 6.32A. The global and target analysis method based 
on a sequential kinetic model plus a constant offset, and convoluted with an instrument response function of $200 \pm 50 \mathrm{fs}$, was used to extract the lifetimes and corresponding evolution associated difference spectra.

\subsection{Quantum-chemistry ground-state computations}

All geometry optimizations were carried out by employing the DFT method, in particular, by making use of the Minnesota DFT/M06-2X hybrid functional and the standard $6-31++\mathrm{G}(\mathrm{d}, \mathrm{p})$ basis set, as implemented in the Gaussian 09 software package. This computational method provided satisfactory descriptions of the reactivity of other DNA-based open-shell and closedshell systems. Frequency calculations by using the harmonic oscillator approximation were used to obtain the ZPVEs and to verify the nature of the stationary points, by checking the absence of any imaginary frequency at the minima and the presence of only one imaginary vibrational mode (reaction coordinate) at the TS structure. For $\mathbf{T}<>\mathbf{A Z} \mathbf{T}^{\bullet+}$, IRC calculations were performed to ensure the connectivity between the TS and related minima. For $\mathbf{T}<>\mathbf{A Z T}^{\mathbf{\bullet}}$, the TS that led to $\mathrm{C}-\mathrm{N}$ bond breaking was not found because the reaction coordinate most probably depended on bond stretching and on a complex molecular reorganization of the relative orientations of the aromatic rings, as revealed by preliminary relaxed scan calculations. Therefore, MEP calculations starting from the two highest points of the relaxed scan potential energy surfaces (C-N distances of 1.85 and $1.90 \AA$, respectively) were conducted to connect both reactants and products, and estimate an upper bound for the energy barrier. The energy of the abovementioned molecular reorganization taking place between the two selected structures was tracked by means of the LIIC procedure, which provided a connected (and therefore, possible) path between the two geometries, thereby ensuring the absence of any energy barrier not found by the relaxed scan exploration. VIPs were computed by subtracting the energies of the neutral state from the energy of the cationic state at the equilibrium geometry of the former, whereas the AIPs were determined by subtracting the energy of the neutral state 
at its corresponding equilibrium geometries from the energy of the cationic state at its equilibrium geometry. On the other hand, the AEAs were obtained by subtracting the energy of the anionic state at its optimized geometry from the energy of the neutral state at its equilibrium geometry. Thereby, positive AEAs indicated stable anionic states, whereas positive VIPs and AIPs referred to unstable cations.

The CASSCF method was used to build multiconfigurational ground-state wave functions on top of the M06-2X geometries. The active space was chosen by including the most relevant out-of-plane nitrogen lone pairs $\left(\mathrm{n}_{\mathrm{N}}\right)$ and $\pi$ and $\pi^{*}$ molecular orbitals of the 6-azauracil and thymine moieties in the azaU-T $\pi$-stacked arrangement, that is, 12 electrons distributed into 12 molecular orbitals (12-in-12) for the neutral system. Thus, active spaces of (13-in-12) and (11-in-12) were used throughout to study the reactivity of the anionic and cationic systems, respectively. In the case of $\mathbf{T}<>\mathbf{A Z} \mathbf{T}^{\bullet-}$ at the reactant structure, one $\pi$ and one $\pi^{*}$ orbital combined to produce the corresponding $\sigma$ and $\sigma^{*}$ orbitals of the C-N bond. Similarly, at the reactant structure of the $\mathbf{T}<>\mathrm{AZT}^{\bullet+}$, two $\pi$ and two $\pi^{*}$ orbitals gave rise to the two $\sigma / \sigma^{*}$ pairs of the $\mathrm{C}-\mathrm{N}$ and the $\mathrm{C}-\mathrm{C}$ covalent bonds.

CASPT2 was used to compute the dynamic electron correlation on top of the CASSCF wave function, and thus, provided accurate energies. An imaginary level shift of 0.2 a.u. was set to minimize the presence of weak intruder states. Regarding the IPEA parameter, the recommended value of 0.25 a.u. was used for the charged systems, as previously benchmarked for cationic and anionic DNA nucleobases, whereas the standard zeroth-order Hamiltonian (IPEA=0.0 a.u.) was employed for the neutral systems. All multiconfigurational calculations were performed with the MOLCAS8 suite of programs and the atomic natural orbital (ANO) L-type basis set with the C,N,O (4s 3p 1d)/H (2s 1p) contraction scheme (ANO-L 431/21). 
Chapter 8:

General Conclusions 

In this Doctoral Thesis, the photocycloreversion process of azetidines is addressed to get more insights into the proposed repair mechanism of (6-4) photoproducts by photolyases. Indeed, the processes involved in this mechanism are still under discussion, being a possible hypothesis the formation and cycloreversion of a fourmembered ring heterocycle. Here, models of the highly unstable azetidine intermediate, proposed for the repair of 6-4PP at TC sequences, have been synthetized to investigate for the first time both the photoinduced reductive and oxidative process. In Chapter 3, stable azabipyrimidinic azetidines obtained from 6-azauracil and thymine or cyclohexene were synthetized in order to determine the influence of thymine moiety in the photoreduction process. Fluorescence, cyclic voltammetry, HPLC and theoretical studies showed that the injection of one electron into the azetidine derived from thymine can occur and leads to a clean cycloreversion that "repairs" the nucleobases. This points to the importance of the thymine moiety that favors a delocalization of the extra electron over the two carbon atoms. For the photooxidative process, in Chapter 4, it was evidenced that the presence of a nitrogen atom in the fourmembered ring affects the redox potential being more favorable the electron transfer for the azetidine compounds than for the related cyclobutane thymine dimer.

Moreover, the study was extended to the ability of the intrinsic photoreductant OG to act as an artificial photolyase. First, in Chapter 5, the photocycloreversion of four-membered model systems bearing an oxetane or a cyclobutane thymine dimer as electron accepting moiety, covalently attached to the photoreductant, were addressed through photochemical and photophysical studies. Then, in Chapter 6, the azetidine ring was incorporated for the first time into an oligonucleotide. Photochemical studies revealed that electron migration is taking place between OG inserted in the opposite strand to the azetidine, splitting the ring and yielding the restored 6-azauracil and thymine original bases. Finally, the ability of (6-4) and CPD photolyases to recognize the azetidines derived oligonucleotide and achieve its repair is under study at this moment. 
To summarize, this doctoral Thesis has provided solid bases to develop the photochemical, photophysical, photobiological study of the oxidative and reductive photocycloreversion of an azetidine. The synthesized models show interesting characteristics as "mimic" of the unstable intermediates involved in the repair of 6-4PP damage by the photolyase. This way, it has been established that these heterocycles can be "repaired" by photoreductants with a reduction potential in the excited state close to that of the reduced flavin, the active cofactor of photolyase. Therefore, photolyases must be capable to cyclorevert the azetidine proposed as a potential intermediate during the repair of (6-4) photoproduct at TC sequences. The study of our azetidine model inserted in an oligonucleotide, which is under study, should provide more details about this mechanism. 
Summary-Resumen-Resum 



\section{Summary}

Ultraviolet radiation is associated with the formation of certain lesions in the DNA that are at the origin of skin cancer. Among the most relevant are the damages that occur at pyrimidine bases: cyclobutane dimers (CPDs) and (6-4) photoproducts (6-4) (6-4PPs). To obtain protection from DNA photolesions, living organisms have enzymes that restore the lesions to their original form, thus maintaining genetic integrity. In some organisms, CPDs and 6-4PPs show an additional repair process, which corresponds to photoreactivation and involves enzymes called CPD and (6-4) photolyases. In particular, there is currently a lively discussion about the mechanism of repair by (6-4) photolyase. The general objective of this doctoral thesis has been to study the cycloreversion of the proposed intermediate of 6-4PP lesions as a key to support one of the mechanisms proposed so far.

In a first place, a model of the intermediate azetidine of the 6-4PP lesion for TC sequences was prepared to investigate its repair by means of electron donation by photosensitizers with suitable redox potential, mimicking the flavin cofactor of the (64) photolyase. Electrochemical, spectroscopic, analytical measurements as well as computational studies showed that the injection of an electron into the azetidine ring leads to a cycloreversion of the bipyrimidine azetidine to the thymine and 6-azauracil bases. It has also been shown that electron transfer only takes place if the thymine component is present in the model.

Secondly, the cycloreversion of azetidine has been investigated by means of an oxidative process in which the azetidine ring donates an electron to the photosensitizer. The comparison with a cyclobutane derivative showed that the presence of the nitrogen in the four-membered ring decreases the redox potential, facilitating thus the oxidation process. 
Third, the cycloversion step has been studied with two intrinsic photosensitizers, guanine and the oxidatively generated damage 8-oxoguanine (OG), covalently bound to a CPD or to an oxetane, as a model for the intermediate of 6-4PP repair. Altogether, the spectroscopic and analytical data showed that these endogenous photosensitizers can act as electron donors mimicking, thus, the function of the flavin cofactor in photolyase.

Finally, azetidine ring has been incorporated in an oligonucleotide to study its cycloreversion by electron transfer. Based on the results of the previous chapter, OG has been chosen as a natural photoreductant. In a first step, a methodology has been developed to insert the azetidine within the oligonucleotide sequence. Then, steadystate irradiation of the duplex containing OG and the azetidine has demonstrated that the electron transfer takes place and leads to the cycloreversion of the heterocycle. In addition, preliminary experiments have been carried out to evaluate the repair of this four-membered ring, as an analog to the (6-4) photoproduct intermediate, by real CPD and (6-4) photolyases.

\section{Resumen}

La radiación ultravioleta está asociada a la formación de ciertas lesiones en el ADN que están en el origen del cáncer de piel. Entre las más relevantes se encuentran los daños que se producen en las bases pirimidínicas: los dímeros ciclobutánicos (CPDs) y fotoproductos (6-4) (6-4PPs). Como protección contra las fotolesiones del $\mathrm{ADN}$, los organismos vivos disponen de enzimas que restauran las lesiones a su forma original, manteniendo así la integridad genética. Algunos organismos manifiestan un proceso de reparación adicional de los CPDs y los 6-4PPs, que corresponde a la fotorreactivación y que involucra enzimas denominadas fotoliasas CPD y (6-4). En concreto, actualmente existe una viva discusión sobre el mecanismo de reparación por la fotoliasa (6-4). El objectivo general de esta tesis doctoral ha sido estudiar la ciclorre- 
versión de los intermedios clave propuestos para la lesión 6-4PP para apoyar uno de los mecanismos propuestos hasta ahora.

En primer lugar se ha preparado un modelo de la azetidina intermedia de la lesión 6-4PP en secuencias TC para investigar su reparación mediante un proceso de donación de electrones a través de fotosensibilizadores con un potencial redox adecuado, mimetizando así el cofactor flavina de la fotoliasa (6-4). Los estudios de electroquímica, espectroscopía, análisis y química computacional mostraron que la posibilidad de inyectar un electrón al anillo de la azetidina conlleva una ciclorreversión de la azetidina bipirimidínica generando las bases timina y 6-azauracilo. También se ha evidenciado que la transferencia de electrones sólo tiene lugar si el componente timina está presente en el modelo.

En segundo lugar, se ha investigado la ciclorreversión de la azetidina mediante un proceso oxidativo en cual el anillo de azetidina dona un electrón al fotosensibilizador. La comparación con un derivado ciclobutánico mostró que la presencia del nitrógeno en el anillo de cuatro miembros disminuye el potencial redox facilitando el proceso de oxidación.

En tercer lugar, el paso de ciclorreversión se ha estudiado con dos fotosensibilizadores intrínsecos, la guanina y el daño oxidativo 8-oxoguanina (OG), unidos covalentemente a un CPD o a un oxetano, como modelo del intermedio formado en la reparación del daño (6-4). En conjunto, los datos de espectroscopía y análisis cromatográfica mostraron la posibilidad de que estos fotosensibilizadores endógenos pueden actuar como dadores de electrones mimetizando, por tanto, la función del cofactor flavina en la fotoliasa.

Finalmente, el anillo de azetidina ha sido incorporado en un oligonucleótido para estudiar su ciclorreversión mediante transferencia de electrones. En base a los resultados de los capítulos previos, OG ha sido elegido como un fotorreductor natural. En un primer paso, una metodología ha sido desarrollada para insertar la azetidina dentro 
de una secuencia de oligonucleótido. Luego, la irradiación en estado estacionario del dúplex que contiene OG y la azetidina ha demostrado que la transferencia de electrones tiene lugar y conlleva a la ciclorreversión del heterociclo. Además, experimentos preliminares han sido llevados a cabo para evaluar la reparación del anillo de cuatro miembros, como un análogo del intermedio generado en el caso del fotoproducto (64), por las fotoliasas reales CPD y (6-4).

\section{Resum}

La radiació ultravioleta està associada a la formació de certes lesions en l'ADN que podrien concluir al càncer de pell. Entre les més rellevants es troben els danys que es produïxen en les bases pirimidínicas: els dímers ciclobutánics (CPDs) i els fotoproductes (6-4) (6-4PPs) . Per a protegir-se de les fotolesions al l'ADN, els organismes vius disposen d'enzims que restauren les lesions a la seua forma original, mantenint així la integritat genètica. En alguns organismes els CPDs i els 6-4PPs manifesten un procés de reparació addicional, que correspon a la fotorreactivació on están involucrats enzims denominades fotoliases CPD i (6-4). En concret, actualment hi ha una viva discussió sobre el mecanisme de reparació per la fotoliasa (6-4). L'objectiv general d'esta tesi doctoral ha sigut estudiar la ciclorreversió dels intermedis clau proposats per a la lesió 6-4PP com a recolzament d' un dels mecanismes proposats fins ara.

En primer lloc s'ha preparat un model de l'azetidina intermèdia de la lesió 6-4PP en seqüències TC per a investigar la seua reparació per mitjà d'un procés de donació d'electrons per fotosensibilizadors amb un potencial redox adequat, mimetitzant així el cofactor flavina de la fotoliasa (6-4) . Els estudis d'electroquímica, espectroscòpia, anàlisi cromatogràfica i química computacional van mostrar que la possibilitat d'injectar un electró a l'anell de l'azetidina comporta una ciclorreversió de l'azetidina bipirimidínica a les bases de timina i 6-azauracil. També s'ha evidenciat que la transferència d'electrons només té lloc si la base timina està present en el model. 
En segon lloc, s'ha investigat la ciclorreversión de l'azetidina mitjacant procés oxidatiu en que l'anell d'azetidina dóna un electró cap al fotosensibilizador. La comparació amb un derivat ciclobutánic va mostrar que la presència del nitrògen en el anell de quatre membres disminuïx el potencial redox facilitant el procés d'oxidació.

En tercer lloc, el pas de ciclorreversió s'ha estudiat amb dos fotosensibilizadors intrínsecs, guanina i el dany oxidatiu 8-oxoguanina (OG), units covalentment a un CPD o a un oxetano, com a intermedi del dany (6-4) . En conjunt, les dades d'espectroscòpia $i$ anàlisi cromatogràfica van mostrar la possibilitat que estos fotosensibilizadors endògens poden actuar com a donadors d'electrons mimetitzant, per tant, la funció del cofactor flavina en la fotoliasa.

Finalment, l'anell d'azetidina ha sigut incorporat en un oligonucleotid per a estudiar la seua ciclorreversió per mitjà de una transferència electrònica. Basant-se en els resultats dels capítols previs, OG ha sigut triat com un fotorreductor natural. En un primer pas, una metodologia ha sigut desenvolupada per a inserir l'azetidina dins d'una seqüència d'oligonucleòtid. Després, la irradiació en estat estacionari del dúplex que conté OG i l'azetidina ha demostrat que la transferència d'electrons té lloc i comporta a la ciclorreversió de l'heterocicle. A més, experiments preliminars han sigut duts a terme per a avaluar la reparació de l'anell de quatre membres, com un anàleg del intermedi en la reparació del fotoproducte (6-4), per les fotoliases reals CPD i (6-4). 



\section{Scientific Contribution}





\section{Contributions to congresses}

1. Fraga-Timiraos, A. B.; Lhiaubet-Vallet, V.; Miranda, M. A. "Synthesis and photoreduction of an azetidine model to mimic (6-4) photoproduct repair". 16th Congress of the European Society for Photobiology, Aveiro, Portugal, 31 August-4 September, 2015. Poster.

2. Fraga-Timiraos, A. B.; Lhiaubet-Vallet, V.; Miranda, M. A. "Repair of a dimeric azetidine related to thymine-cytosine (6-4) photoproduct by electron transfer photoreduction". XXVI Biennial Meeting of Organic Chemistry, Punta Umbría, Spain, June 14-17th, 2016. Poster and Flash communication.

3. Fraga-Timiraos, A. B.; Lhiaubet-Vallet, V.; Miranda, M. A. "Photorepair of pyrimidine dimers by 8-oxo-guanosine as a flavin mimic". XXXVI Biennial Meeting of the Royal Spanish Society of Chemistry, Sitges, Spain, June 2529th, 2017. Poster.

\section{Publications}

1. Fraga-Timiraos, A. B.; Francés-Monerris, A.; Rodríguez-Muñiz, G. M.; Navarrete-Miguel, M.; Miranda, M. A.; Roca-Sanjuán, D.; Lhiaubet-Vallet, V. "Experimental and theoretical study on the cycloreversion of a nucleobasederived azetidine by photoinduced electron transfer", Chem. Eur. J. 2018, 24, 15346-15354.

2. Fraga-Timiraos, A. B.; Lhiaubet-Vallet, V.; Miranda, M. A. "Repair of a dimeric azetidine related to thymine-cytosine (6-4) photoproduct by electron transfer photoreduction", Angew. Chem. Int. Ed. 2016, 55, 6037-6040.

3. Fraga-Timiraos, A. B.; Rodríguez-Muñiz, G. M.; Peiro-Penalba, V.; Miranda, M. A.; Lhiaubet-Vallet, V. "Stereoselective fluorescence quenching in the electron transfer photooxidation of nucleobase-related azetidines by cyanoaromatics", Molecules, 2016, 21, 1683-1691. 